Linköping Studies in Science and Technology

Dissertation No. 2168

\title{
Security-Aware Design of Cyber-Physical Systems for Control Applications
}

\section{Rouhollah Mahfouzi}

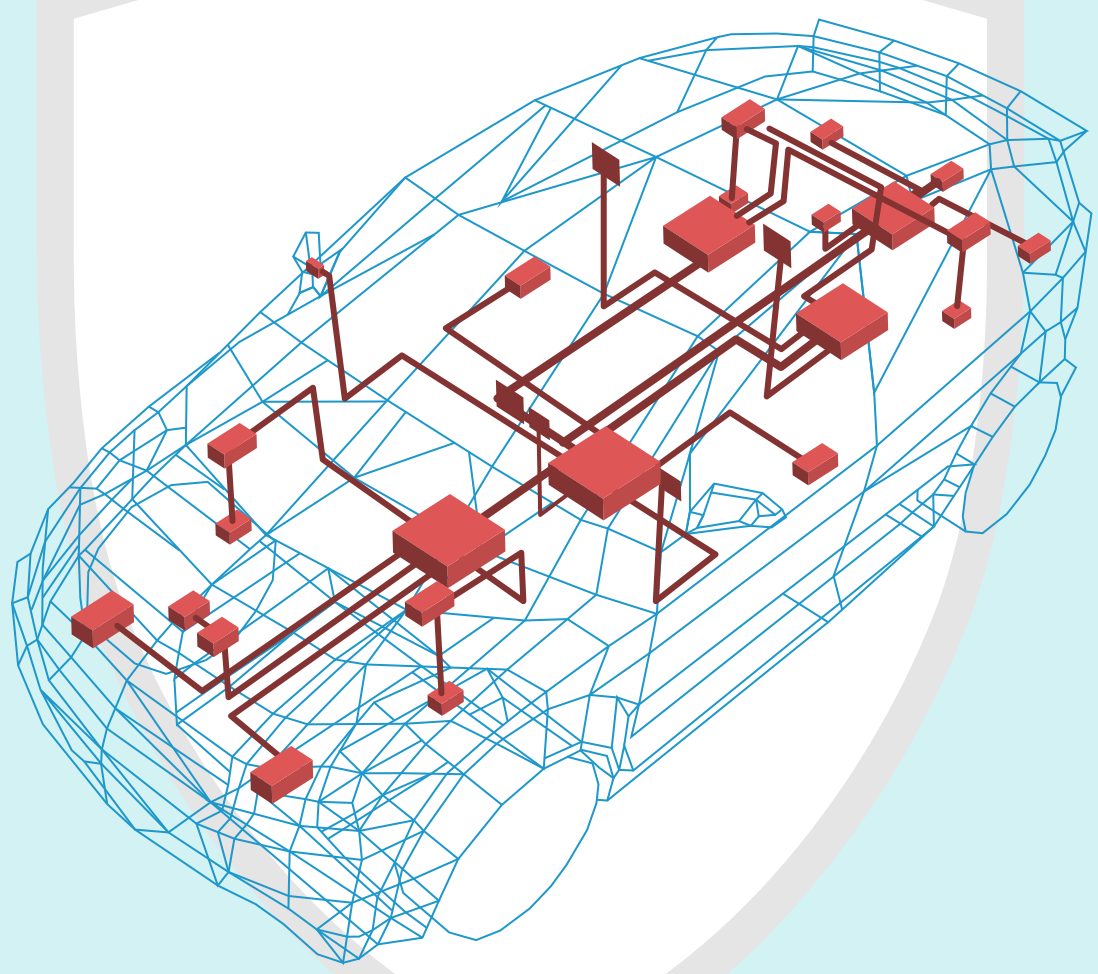



Linköping Studies in Science and Technology

Dissertations, No. 2168

\title{
Security-Aware Design of Cyber-Physical Systems for Control Applications
}

\author{
Rouhollah Mahfouzi
}

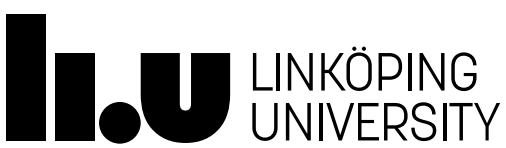

\author{
Linköping University \\ Department of Computer and Information Science \\ Division of Software and Systems \\ SE-581 83 Linköping, Sweden
}

Linköping 2021 


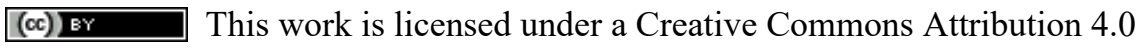
International License.

https://creativecommons.org/licenses/by/4.0/

Edition 1:1

(C) Rouhollah Mahfouzi, 2021

ISBN 978-91-7929-021-4

ISSN 0345-7524

URL http://urn.kb.se/resolve?urn=urn:nbn:se:liu:diva-178320

Published articles have been reprinted with permission from the respective copyright holder.

Typeset using $\mathrm{AT}_{\mathrm{E}} \mathrm{X}$

Printed by LiU-Tryck, Linköping 2021 


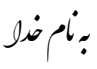
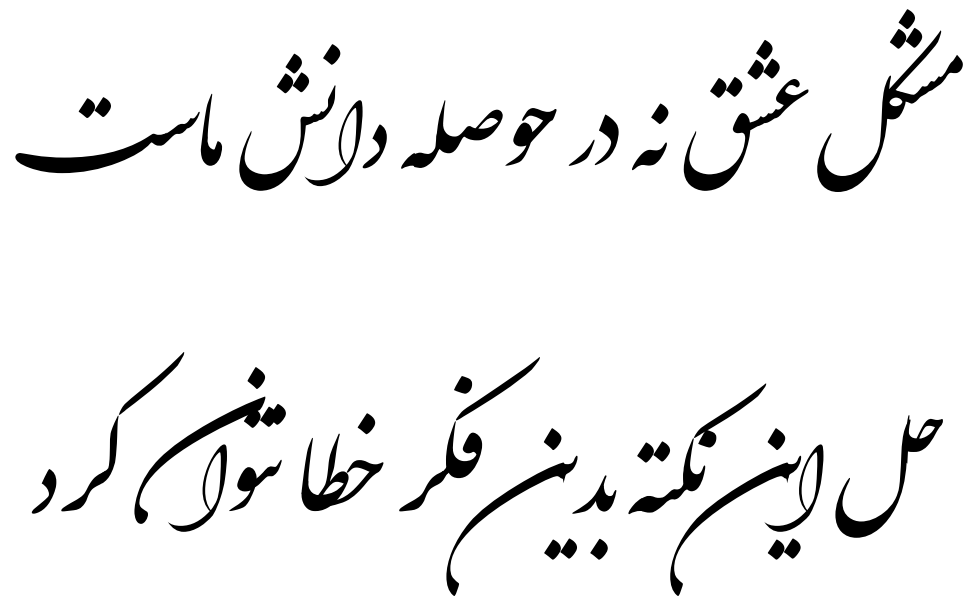

$\dot{b} 6$

The difficulty of love is not in the capacity of our knowledge with this mistaken thought one cannot loosen this subtlety. 

To My Family 



\section{POPULÄRVETENSKAPLIG SAMMANFATTNING}

Cyberfysiska system interagerar med den fysiska och digitala världen. Cyberfysiska system innehåller ofta ett flertal styrsystem. Dessa system är uppkopplade till Internet och är därför särskilt sårbara mot cyberattacker. Flera attacker är särskilt riktade mot styrsystem. Denna avhandling fokuserar på säkerhetshot mot styrsystem i cyberfysiska system. Vi presenterar unika tillstånd för styrsystem som används till att utveckla system för att upptäcka, förhindra och mildra attacker mot cyberfysiska system. Vi påvisar även säkerhetsutmaningar baserade på icke-intuitiva tids- och fördröjningsberoenden som skapas då ett flertal styrsystem delar resurser. Vidare så tar vi hänsyn till realtidskommunikation för reglerapplikationer som implementeras på ett underliggande datornätverk där vi schemalägger och dirigerar meddelanden på så sätt att säkerheten maximeras. Till sist behandlar vi säkerhetsutmaningar som uppstår då reglerapplikationer i cyberfysiska system allokeras som molntjänster via Internet. Denna avhandling innehåller experiment som påvisar effektiviteten av verifierbar beräkning för att i realtid verifiera molntjänstens beräkningar. 


\section{ABSTRACT}

With cyber-physical systems opening to the outside world, security can no longer be considered a secondary issue. In this work, we focus on security threats to control applications in cyber-physical systems. We provide detection, prevention, and mitigation solutions to attacks considering the stringent resource constraints and important properties of such systems.

First, we highlight some important properties of control applications that are used to design an intrusion detection and mitigation mechanism. We show how the control laws, derived from the physical properties of control applications, can facilitate the intrusion detection mechanism. We also use a resource management approach to maintain the performance of the control application under attack.

Second, we elaborate on the challenges derived from sharing a processor among several controller tasks. We investigate the counter-intuitive timing anomalies that result from such resource sharing and introduce the Butterfly attack which exploits these anomalies. With the Butterfly attack, the adversary interferes with a low criticality and less protected task to change the timing behavior of the other tasks sharing the same platform. We experimentally show how this attack can indirectly destabilize a high criticality and, potentially, more protected task.

Then, we consider real-time communication of control applications over a Time-Triggered Ethernet network. We demonstrate the impact of varying delays on control stability and identify the route and schedule constraints that are necessary to guarantee stability. On top of that, we study the impact of encryption and decryption delays on stability and employ a design space exploration approach to maximize security while continuing to satisfy stability guarantees. 
Finally, we focus on the security challenges of control systems in which the control algorithm is outsourced to the cloud. We highlight the necessity of a verification mechanism that enables the physical plant to check the correctness of the calculations performed in the cloud. We experimentally demonstrate the effectiveness of a verifiable computation technique in the context of advanced control methods, such as Model Predictive Control (MPC), deployed over the cloud. 



\section{Acknowledgments}

I want to express my sincere gratitude to Petru Eles who has been an ideal advisor, offering valuable advice with a perfect blend of insight. I cannot thank Petru enough for his encouragement, patience, and support. I would also like to thank Zebo Peng, an encouraging co-advisor and a patient, motivating, and supportive leader. The friendly and constructive work environment in Embedded Systems Group is indebted to Petru and Zebo's leadership.

I would like to thank my co-advisor and friend Amir Aminifar for all the guidance and help both in academic and private life. Taking the first steps in my new academic life was much harder without Amir's input. I also appreciate my co-advisor Soheil Samii for giving helpful comments in discussions and delivering industrial insight into this work.

Throughout this journey, I took advantage of many knowledgeable people. My gratitude goes to Ahmed Rezine for all the great scientific and non-scientific discussions. I appreciate Mattias Villani and Mathias Payer for sharing their knowledge and contributing to this thesis. I gratefully recognize the help of Anton Cervin from the Automatic Control Department of Lund University for his help and support in the control-related parts of this thesis. It was a pleasure to collaborate with Anton, Karl-Erik Årzén, and other members of the Automatic Control Department of Lund University.

I am fortunate to have been a part of the Embedded Systems Group. I would like to thank the former and current members of the group especially, Adrian, Ali, Arian, Ivan, Mina, Yuanbin, Yungang, and Zeinab for inspiring group meetings, enjoyable coffee breaks, and fruitful lunch discussions. 
I would like to thank Brittany Shahmehri for proofreading this thesis. I also thank the administrative staff at IDA, especially Lene Rosell, Anne Moe, and Linda Strandman for facilitating my work at IDA.

I want to thank Mahdi Morsali and Meysam Aghighi for all the delightful discussions and enjoyable moments both in the workplace and in private life. Their company helped me adjusting work/life balance. I appreciate Biman Roy and Antonia Arvanitaki for all the open and honest discussions and for being available for short talks.

My foremost gratitude goes to my beloved wife Zahra. Without her caring, kindness, and patience this accomplishment was impossible. I greatly appreciate her sacrifices and will never forget her efforts. Thank you, Zahra! I am also thankful for all the joy and fun my two sons Mohsen and Amir brought to our life. Waking up to a happy and smiling family is what I enjoy the most.

Last but not least, I want to deeply thank my parents for all the love and care they gave me during my life. Every accomplishment I make is indebted to their love and support. Thank you, Mom! Thank you, Dad!

Rouhollah Mahfouzi

Linköping, Sweden, 2021 


\section{Contents}

Abstract vii

Acknowledgments xii

Contents xiii

List of Figures $\quad$ xvii

List of Tables $\quad$ xxi

1 Introduction $\quad 1$

1.1 Motivation .................... 1

1.2 Research Questions ................. 3

1.3 Related Work . . . . . . . . . . . . . . . 6

1.4 Summary of Contributions . . . . . . . . . . . . . 10

1.4.1 Intrusion Detection and Mitigation for Control Applications Under Observable and Controllable Attacks . . 11

1.4.2 Temporal Attacks on Control Applications . . . . . . . 12

1.4.3 Security and Stability-Aware Routing and Scheduling of Ethernet TSN Messages . . . . . . . . . . . . . . . 12

1.4.4 Secure Cloud-Based Control Using Verifiable Computation ................... . 13

1.5 List of Publications . . . . . . . . . . . . . . 13

2 Background and Preliminaries $\quad \mathbf{1 5}$

2.1 System Model . . . . . . . . . . . . . . . . . . . . . . . . . . . . 16

2.1.1 Shared Processor . . . . . . . . . . . . 16 
2.1.2 Shared Network . . . . . . . . . . . . . 17

2.1.2.1 Ethernet TSN Switches . . . . . . . . . 18

2.1.3 Plant Model . . . . . . . . . . . . . . . . . . . 19

2.2 Control Performance . . . . . . . . . . . . . . . . . 20

2.3 Stability Analysis . . . . . . . . . . . . . . 22

2.4 Model Predictive Control . . . . . . . . . . . . . . . . . . 25

2.5 Confidentiality and Integrity . . . . . . . . . . . . . 27

2.5.1 Authenticated Encryption . . . . . . . . . . 28

3 Intrusion Detection and Mitigation for Control Applications 29

3.1 Introduction and Related Work . . . . . . . . . . . 30

3.1.1 Important Properties of Control Applications . . . . . 33

3.1.2 Observable and Controllable Attacks . . . . . . . . . . 34

3.1.3 Intrusion-Damage Assessment and Mitigation . . . . . 34

3.1.3.1 Intrusion-Damage Assessment . . . . . . . . 34

3.1.3.2 Intrusion-Damage Mitigation . . . . . . . 35

3.2 System Model . . . . . . . . . . . . . . . . 36

3.2.1 Control Task Model . . . . . . . . . . . . . . 36

3.3 Attack Model . . . . . . . . . . . . . . . . . . 37

3.4 Motivational Example . . . . . . . . . . . . . . . . 39

3.5 Problem Formulation . . . . . . . . . . . . . . . . . . 43

3.6 Approach . . . . . . . . . . . . . . . 44

3.6.1 Intrusion-Damage Assessment . . . . . . . . . . . . . . 45

3.6.1.1 Offline Learning . . . . . . . . . . . . . 46

3.6.1.2 Online Evaluation . . . . . . . . . . . 47

3.6.2 Intrusion-Damage Mitigation . . . . . . . . . . . . 48

3.6.2.1 Desired Sampling Unit . . . . . . . . . . . . 49

3.6.2.2 Resource Management Unit . . . . . . . . 50

3.6.3 Experimental Example . . . . . . . . . . . . . 50

3.6.3.1 Experimental Setup . . . . . . . . . . . 51

3.6.3.2 Evaluation of Intrusion-Damage Assessment 51

3.6.3.3 Evaluation of Intrusion-Damage Mitigation . 54

3.7 Conclusions . . . . . . . . . . . . . . . . . 57

4 Temporal Attacks on Control Applications 59

4.1 Introduction and Related Work . . . . . . . . . . . . 60 
4.2 System Model . . . . . . . . . . . . . . . . . . . . . . 64

4.3 Worst-Case and Best-Case Response Time . . . . . . . . . . . 65

4.4 Scheduling Parameters and Control Performance . . . . . . . . 66

4.4.1 Changing Sampling Period . . . . . . . . . . . . . 67

4.4.2 Changing Priority . . . . . . . . . . . . . 69

4.4.3 Changing Computation Time . . . . . . . . . . . . 69

4.5 Threat Model . . . . . . . . . . . . . . . . . 70

4.6 Butterfly Attack . . . . . . . . . . . . . . 72

4.7 Case Study 1: Automotive Application . . . . . . . . . . . . . 74

4.8 Case Study 2: Unmanned Aerial Vehicle (UAV) . . . . . . . . 78

4.9 Discussion . . . . . . . . . . . . . . . . . . . 85

4.10 Conclusion . . . . . . . . . . . . . . . . . . . 88

$5 \quad$ Security and Stability-Aware Routing and Scheduling $\quad 89$

5.1 Introduction and Related Work . . . . . . . . . . . . . . . . 90

5.2 Preliminaries . . . . . . . . . . . . . . . . 93

5.2.1 Security Level . . . . . . . . . . . . . . . . . 93

$5.2 .2 \quad$ Stability Analysis . . . . . . . . . . . . . . . . 98

5.3 System Model . . . . . . . . . . . . . . . . . . . . . . . . 98

5.3.1 Network and Ethernet Switches . . . . . . . . . . . . . 99

5.3 .2 Delay and Jitter . . . . . . . . . . . . . . . 100

5.3.3 Control Applications . . . . . . . . . . . . . . . . 101

5.3.4 Security Model . . . . . . . . . . . . . . . . . 101

5.4 Threat Model . . . . . . . . . . . . . . . . 102

5.5 Problem Formulation . . . . . . . . . . . . . . . . . 103

5.6 Motivational Example . . . . . . . . . . . . . . . . . . . 105

5.6.1 Routing and Scheduling without Security Constraints 106

5.6.2 Routing and Scheduling with Security Constraints . . 110

5.7 Stability-Aware Routing and Scheduling . . . . . . . . . . . 115

5.7.1 Constraints With Respect to Routing and Scheduling . 115

5.7 .2 Stability Constraints . . . . . . . . . . . . 116

5.7 .3 Improving scalability . . . . . . . . . . . . . . 117

5.7.3.1 Route Subset . . . . . . . . . . . . 117

5.7.3.2 Incremental Synthesis . . . . . . . . . . . . . 118

5.7.4 Experimental Setup and Results . . . . . . . . . 118

5.8 Security and Stability-Aware Routing and Scheduling . . . . . 124 
5.8.1 Constraints with Respect to Routing and Scheduling . 125

5.8.2 Stability Constraints . . . . . . . . . . . . 125

5.8.3 Security Optimization . . . . . . . . . . . . 125

5.8.4 Improving Scalability . . . . . . . . . . . . . . . 127

5.8.4.1 Binary Search . . . . . . . . . . . 127

5.8.4.2 Genetic Algorithm . . . . . . . . . 128

5.8.5 Experimental Setup and Results . . . . . . . . . 129

6 Secure Cloud-Based Control Using Verifiable Computation 139

6.1 Introduction and Related Work . . . . . . . . . . . . . 140

6.2 Verifiable Computation . . . . . . . . . . . . . . . . 143

6.3 System Model . . . . . . . . . . . . . . . . . . . 145

6.4 Threat Model . . . . . . . . . . . . . . . . 146

6.5 Implementation of MPC using Pequin . . . . . . . . . . . . . 147

6.6 Case Studies . . . . . . . . . . . . . . . . . . . . . . . . . . . . 148

6.6.1 Case 1: Helicopter System . . . . . . . . . . . . 148

6.6.2 Case 2: Water Tank System . . . . . . . . . . . . 150

6.7 Physical Water Tank Plant . . . . . . . . . . . . . . . 154

6.8 Impact of Control Parameters and Cloud Server Performance . 156

6.9 Discussion . . . . . . . . . . . . . . . . 158

6.10 Conclusion . . . . . . . . . . . . . . 159

7 Conclusions and Future Work 161

7.1 Conclusions . . . . . . . . . . . . . . . 162

7.2 Future Work .................... 163

$\begin{array}{lr}\text { Bibliography } & 167\end{array}$ 


\section{List of Figures}

2.1 Control applications sharing a processing unit. . . . . . . . . . . . 17

2.2 Control applications sharing a communication network. . . . . . . 18

2.3 Overview of an Ethernet TSN switch. . . . . . . . . . . . . . 19

2.4 Control cost vs. the sampling period for a constant delay. . . . . . 21

2.5 Control cost vs. the delay. . . . . . . . . . . . . . . . . 22

2.6 Graphical interpretation of the latency and worst-case jitter. . . . 23

2.7 Stability curve generated by Jitter Margin tool. . . . . . . . . . . 24

2.8 Basic illustration of predictive horizon. $r(k)$ is the reference trajectory, $y(k)$ is the controlled output and $u(k)$ represents the control signal. . . . . . . . . . . . . . . . . . . . 26

3.1 Two control applications sharing the same platform. . . . . . . . 39

3.2 Schematic drawing of the inverted pendulum on a cart. . . . . . . 40

3.3 Control cost of pendulum under different attacks. . . . . . . . . . 42

3.4 Overview of our approach. . . . . . . . . . . . . . . . . . 44

3.5 Intrusion-generated damage; no intrusion mitigation in operation. 52

(a) Discard 4-10 actuator commands. . . . . . . . . . 52

(b) Manipulate sensor readings. . . . . . . . . . . . . 52

3.6 Deviation of the pendulum $P_{1}$ from upright position (angle $\theta$ ) during two attack scenarios; no intrusion mitigation in operation. 52

(a) Discard 4-10 actuator commands. . . . . . . . . . 52

(b) Manipulate sensor readings. . . . . . . . . . . . . . 52

3.7 Control cost for the pendulum $P_{1}$ during two attack scenarios; no intrusion mitigation in operation. . . . . . . . . . . . 53

(a) Discard 4-10 actuator commands. . . . . . . . . . 53

(b) Manipulate sensor readings. . . . . . . . . . . . 53 
3.8 Intrusion-generated damage calculated for the two pendulums during two attack scenarios while intrusion mitigation is functioning. $\quad 55$

(a) Discard 10 actuator commands. . . . . . . . . . 55

(b) Manipulate sensor readings. . . . . . . . . . . . 55

3.9 Deviation of the two pendulums from upright position (angle $\theta$ ) during two attack scenarios while the intrusion mitigation is in operation. . . . . . . . . . . . . . 56

(a) Discard 10 actuator commands. . . . . . . . . 56

(b) Manipulate sensor readings. . . . . . . . . . 56

3.10 Control cost for the two pendulums during two attack scenarios while the intrusion mitigation is in operation. . . . . . . . 56

(a) Discard 10 actuator commands. . . . . . . . . 56

(b) Manipulate sensor readings. . . . . . . . . . 56

3.11 Allocation of resources (in terms of sampling frequency) for the two pendulums during two attack scenarios. . . . . . . . . 57

(a) Discard 10 actuator commands. . . . . . . . . 57

(b) Manipulate sensor readings. . . . . . . . . . 57

4.1 Illustration of the Butterfly attack scenario. . . . . . . . . . . 63

4.2 Non-monotonicity of jitter w.r.t timing characteristics of other tasks. 68

(a) Original task schedule. . . . . . . . . . . . 68

(b) Increasing period of $\tau_{2}$ to $24 \ldots \ldots$. . . . . . . 68

(c) Decreasing period of $\tau_{2}$ to $6 . \ldots \ldots 6$

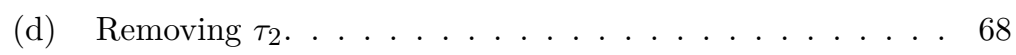

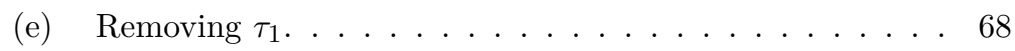

(f) Decreasing computation time of $\tau_{2} \ldots \ldots \ldots$. . . . . 68

(g) Decreasing computation time of $\tau_{1} \ldots \ldots \ldots$. . . . . 68

4.3 Schedule of vehicle positioning and DC motor controller tasks on the shared platform. . . . . . . . . . . . 77

(a) Before increasing the period of task $\tau_{1}$. . . . . . . 77

(b) After increasing the period of task $\tau_{1}$ from 15 to $60 . \ldots 77$

4.4 The stability curve generated by Jitter Margin. . . . . . . . . . . . 78

4.5 Stable DC motor without adversary. . . . . . . . . . . . . 79

(a) DC motor rotational speed (stable). . . . . . . . . 79

(b) DC motor control cost (stable). . . . . . . . . . 79 
4.6 Unstable DC motor in the presence of adversary. . . . . . . . . . . 79

(a) DC motor rotational speed (unstable). . . . . . . . 79

(b) DC motor control cost (unstable). . . . . . . . . . 79

4.7 Schedule of update_GPS and run_nav_updates tasks on the shared platform. The green (update_GPS) task has a higher priority than the red (run_nav_updates) task. The properties of the tasks are $\tau_{1}=(2,4,5), \tau_{2}=(1,1,10) \ldots \ldots \ldots$. . . . 81

(a) Before increasing the period of task $\tau_{1} \ldots \ldots$. . . . . 81

(b) After increasing the period of task $\tau_{1}$ from 5 to $20 . \ldots 81$

4.8 Stable quadcopter without adversary. . . . . . . . . . . . . 82

(a) Quadcopter vertical angle (stable). . . . . . . . . . 82

(b) Quadcopter control cost (stable). . . . . . . . . 82

4.9 Unstable quadcopter in the presence of adversary. . . . . . . . . . 82

(a) Quadcopter vertical angle (unstable). . . . . . . . . 82

(b) Quadcopter control cost (unstable). ......... . 82

4.10 Position of quadcopter during transition. . . . . . . . . . . . . . . 84

(a) Longitude.................... . . 84

(b) Latitude..................... 84

(c) Altitude .................... . 84

4.11 Position of quadcopter in the presence of an adversary. . . . . . . 85

(a) Longitude.................. . . 85

(b) Latitude...................... 85

(c) Altitude .................... 85

4.12 Two-dimensional position of the quadcopter. . . . . . . . . . . 86

5.1 Encryption/Decryption time versus key size for Rijndael. . . . . . 96

5.2 Security level versus key size for Rijndael. . . . . . . . . . . . . . 96

5.3 Encryption/Decryption time versus number of rounds and key size for RC6. . . . . . . . . . . . . . . . . . . 97

5.4 Issue time of messages from control applications. . . . . . . . . . . 106

5.5 Network topology for motivational example. . . . . . . . . . . . 107

5.6 Scalability of the incremental synthesis heuristic. . . . . . . . . . . 119

5.7 Percentage of unsatisfied problems with incremental synthesis. . . 119

5.8 Scalability of the route subset heuristic. . . . . . . . . . . . . 120

5.9 Scalability of the proposed approach for big networks. . . . . . . . 121

5.10 Synthesis time for theoretical optimum and binary search. . . . . . 129 
5.11 Maximum of the minimum security level for the theoretical optimum and binary search. . . . . . . . . . . . . . . 130

5.12 Synthesis time for SMT-LIB optimization and binary search with heuristics $($ routes $=4$, slices $=5) \ldots \ldots \ldots$. . . . . . . . 131

5.13 Maximum of the minimum security level for SMT-LIB optimization and binary search with heuristics (routes=4, slices=5) . . . . 132

5.14 Synthesis time for SMT-LIB optimization and genetic algorithm with heuristics $($ routes $=4$, slices=5) . . . . . . . . . 133

5.15 Maximum weighted sum for SMT-LIB optimization and genetic algorithm with heuristics $($ routes $=4$, slices=5) . . . . . . . . 133

6.1 Overview of the outsourcing scenario. . . . . . . . . . . . . 146

6.2 Schematic of the helicopter system. . . . . . . . . . . . . . . 149

6.3 The elevation and rotation angles. . . . . . . . . . . . . 150

6.4 Round-trip time for helicopter (case 1) and water tank (case 2) system. . . . . . . . . . . . . . . . . . . . 151

6.5 Schematic of the water tank system. . . . . . . . . . . . . . 152

6.6 Water level in the two lower tanks. . . . . . . . . . . . . . . . 153

6.7 Water tank system controlled via cloud. . . . . . . . . . . . . . . . 155

6.8 Quadratic control cost versus prediction horizon. . . . . . . . . . 157

6.9 Time spent in the cloud to calculate output and generate proof for different prediction horizons. . . . . . . . . . . . . . . . 157

6.10 Time spent on the client to verify the proof for different prediction horizons. . . . . . . . . . . . . . . . 157 


\section{List of Tables}

3.1 Specification of pendulum 1 and 2. . . . . . . . . . . . 41

4.1 Scheduling parameters of example tasks. . . . . . . . . . . . 67

5.1 Number of plaintext-ciphertext pairs required to mount a successful attack on RC6. . . . . . . . . . . . . . . . . . . . 97

5.2 Parameters of the example control applications. . . . . . . . . . 106

5.3 Arrival time of messages with FR and IRS approach. . . . . . . . 108

5.4 Security trade-off function for Rijndael. . . . . . . . . . . . . . . . 110

5.5 Arrival time of messages using the naive approach for integrating security. . . . . . . . . . . . . . . . . 111

5.6 Arrival time of messages for maximizing the minimum security level (192 bits). . . . . . . . . . . . . . . . . . . 113

5.7 Arrival time of messages for maximizing the weighted sum of security levels. . . . . . . . . . . . . . . . . . 114

5.8 Parameters of the example control applications and routing and scheduling results. . . . . . . . . . . . . . . . . . 123

5.9 Parameters of the example control applications and routing and scheduling results. . . . . . . . . . . . . . . 136

6.1 Time spent to calculate output and generate proof for various cloud configurations with different amounts of computational power.158 



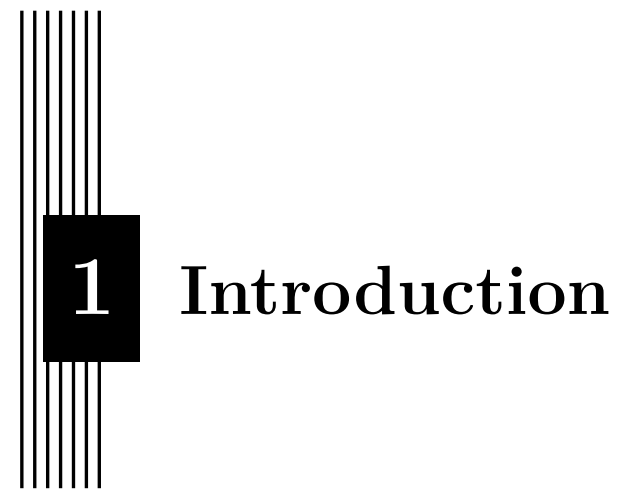

In this chapter, we provide a broad introduction to the topics covered in this thesis. We also describe the research questions we are addressing and highlight the contributions of this thesis.

\subsection{Motivation}

Cyber-physical systems (CPSs) integrate computation, networking, and physical processes and are utilized in many areas, from automotive and avionics to industrial automation and nuclear power plants (Törngren et al., 2017; Wang et al., 2015). With the extension of IoT devices, CPSs are more and more connected to the Internet, which makes them more vulnerable to security attacks. In recent years, many security attacks on industrial and safety-critical systems have taken place (Shafique et al., 2018). The intention of some of these attacks was not only to steal data but to inflict damage to the physical facilities or even take lives. In 2010, a malicious computer worm called Stuxnet was first identified. The original goal of Stuxnet, known as the first cyber-warfare weapon, was to physically destroy an Iranian nuclear facility (Langner, 2011; 
McAfee LLC, 2019). Although Stuxnet is regarded as a landmark in cyberwarfare, there have been many other attacks after Stuxnet against industrial applications (Lindsay, 2013). In the context of the automotive industry, in 2015, hackers showcased a successful remote attack against a brand new Jeep Cherokee (Miller and Valasek, 2015). The attackers were able to remotely control safety-critical functionalities like the braking system and the steering wheel.

A large class of attacks is directed at control systems that are at the heart of CPSs. Although conventional security measures, like network firewalls, intrusion detection and mitigation systems, and anti-malware systems, are available in every security-critical system, these security measures only reduce the cyber risk but cannot prevent all attacks. Moreover, CPSs can be compromised even without an IT-based exploit, in transduction attacks (Fu and $\mathrm{Xu}, 2018$ ), for example by electromagnetically interfering with an actuator (Selvaraj et al., 2018) or spoofing GPS sensors (Kerns et al., 2014). Therefore, when it comes to applications that are controlling a physical plant and that also interact with the physical environment, there should be an extra layer of security (on top of conventional IT-based security measures) implemented in the control application layer to further protect critical control applications from attacks. Moreover, certain characteristics of control applications, such as physics-based modeling of the physical plant and sampling periodicity, differentiate CPSs from regular IT-based systems. Therefore, there is a demand for a particular type of knowledge and additional measures to secure CPSs (Khalid et al., 2020). The focus of this thesis is to utilize the specific characteristics of the control applications to detect and mitigate attacks against CPSs, as well as to design CPSs that are more resilient to attacks.

CPSs work with limited resources and usually host multiple control applications that are implemented on a shared platform. Computation and communication that are performed on shared resources must be scheduled, which makes the control applications interdependent and results in complex timing behavior in control tasks. The violation of certain timing related constraints can lead to reduced control quality, and can even affect the stability of the physical plants. 
Due to resource constraints, as well as power efficiency and safety requirements, the design and synthesis of embedded control applications are challenging. Implementing security measures consumes additional resources and can add even more complexity to the design and synthesis problem. On one hand, the stability and performance of a control application are intertwined with the amount of resources available for that application. On the other hand, security measures, such as encryption and decryption, can consume the limited available resources and deteriorate the control performance. However, security measures are necessary to guarantee the resilience and robustness of control applications. Therefore, the correlation between implementing security measures and allocating resources for control applications must be taken into account, and thus the security measures for control applications must be considered at design time.

The main research question is: how does one introduce security measures during the design and synthesis of control applications that are using a shared platform, and that have stringent resource constraints? In the next section, we are going to elaborate more on this research question.

\section{$1.2 \quad$ Research Questions}

A control system consists of a controller and a physical plant. The controller interconnects with the physical plant via a control loop that is run periodically. In each sampling period, the state of the physical plant is read and sent to the controller, and the controller calculates the appropriate control input based on the sampled information. The control signal is then applied to the physical plant. The main goal of the controller is to keep the physical plant at a steady state. A model of the physical plant, derived from the dynamics of the plant (laws of physics) is used to synthesize the controller. Having the physical model makes it possible to predict the reaction of the physical plant to certain control inputs. That is, the states of the physical plant should follow those that are expected according to the physics of the system. If the control system is under attack and the input/output to/from the physical plant is manipulated, there will be a deviation between the state of the system and the one that is expected based on the laws of physics. This deviation can be used by the controller to detect the attack. 
As a result of shifting towards integrated architectures in the implementation of embedded systems, most control applications are deployed as software tasks on shared platforms. The shared resource is a microprocessor and/or a communication channel. The process of allocating the shared resource to tasks is commonly known as scheduling. Scheduling implies the allocation of processing resources to tasks and allocation of bandwidth capacity to messages transmitted between tasks as well as the decision of the start times to execute the tasks and to send the messages. With a platform that is shared among several tasks, it is possible to dynamically allocate more or fewer resources to different tasks that are utilizing the shared platform. On the other hand, the control performance of the plant strongly depends on the amount of allocated resources. Therefore, it might be possible to allocate more resources to a controller that is under attack in order to mitigate the damages caused by an attacker. Therefore, our first research question is formulated as follows.

\section{- RQ1:}

(a) How can we use the physical properties of the plant to detect anomalies during an attack on a control system?

(b) How can we use the resource management on a shared platform to mitigate an attack against the controller?

Sharing a platform makes the temporal properties of the tasks dependent on each other. Therefore, the change in computation time, priority, and period of one task may result in abnormal timing behavior of another task. On the other hand, the varying delays experienced by a controller task have a dramatic impact on the performance and stability of the control application. Therefore, manipulating the timing behavior of one task may affect the control performance of another task or even destabilize it. The problem becomes more complex if we consider the non-monotonicity characteristic of the timing properties of tasks utilizing a shared platform. That is, counter-intuitively, decreasing the sampling period or increasing the priority of a control task may reduce the control performance or even jeopardize the stability of the physical plant.

- RQ2: Is it possible to destabilize a control application by manipulating the timing behavior of another task utilizing the same shared resource? 
Ethernet is being increasingly used as a shared communication channel in various time-critical CPSs. The scheduling and routing of messages on the shared communication channel determine the latency and jitter experienced by each control task. Therefore, the routing and scheduling of messages strongly impact the stability and performance of the control applications. Finding an implementation that satisfies the stability requirements of all control applications in the system requires joint routing and scheduling. However, the synthesis of schedules and routes over an Ethernet network in the context of real-time control applications is inherently complex. Therefore, complex design-space exploration techniques need to be utilized to jointly synthesize the routes and schedules of messages for such networked control systems.

Using the shared network for sending/receiving messages to/from control applications poses security threats to the confidentiality and integrity of messages. To ensure safety in the presence of adversarial attacks, security measures (i.e., end-to-end encryption/decryption) must be implemented for all messages. Increasing the security level of the encryption/decryption increases the resilience of the control applications to attacks. However, increasing the security level for a message leads to extra delay (due to the increased computational load implied by encryption/decryption) and could result in violation of timing requirements and instability in a control application. Therefore, finding an affordable and sufficient security level for each control application should be considered during synthesis of routes and schedules over the whole system.

\section{- RQ3:}

(a) How is integrated route and schedule synthesis for real-time messages over a time-triggered Ethernet network achieved while guaranteeing certain stability conditions for control applications?

(b) How can the stability of all control applications be guaranteed while maximizing the security of the system?

Cloud computing platforms have recently attracted attention in the CPSs domain, e.g., automotive industry and industrial automation. Outsourcing the control computation to the cloud poses security threats on control applications. In the case of critical control applications, the main security concern is 
untrusted network and cloud providers. Being able to verify the correctness of the result returned from the cloud, without actually redoing the computation, can resolve many of the security concerns. Verifiable computation is a cryptographic protocol that enables the cloud to provide mathematical proof to the client in order to guarantee the integrity of the returned data. Although recent developments in modern cryptography claim that verifiable computation is applicable in real life, the practicality of these techniques in the context of control systems has never been investigated.

- RQ4: To what extend is the verifiable computation approach practical when it comes to cloud deployment of control applications?

\subsection{Related Work}

In this section, we will review the related research contributions from the literature. Based on the surveyed research, we can distinguish our contributions and place our thesis in a global context. Further discussion regarding related work for each contribution will follow in the individual chapters.

The close connection of CPSs with the physical world opens up a variety of security threats to these systems. In the past decades, researchers have looked into the security of CPSs from many different perspectives.

Cyber security in systems connected to physical domains, specifically, Supervisory Control and Data Acquisition Systems (SCADA), has been studied for years (Oman et al., 2000). However, these efforts were focused on applying existing conventional IT security to control systems. Much of the research related to the security of industrial control systems is focused on network and software layers and, therefore, classical IT security solutions have been proposed. As oppose to these approaches we do not consider these categories of papers, rather we focus on certain security issues related to sensing, actuation, and control, as well as on the interplay between scheduling and stability. Therefore, in the scope of this thesis we consider the literature from the past decade that focuses on control systems and lower levels of CPSs, i.e., sensing, actuation, and control. 
Much early work concerning the security of CPSs was devoted to identifying the specific security related characteristics of CPSs (Anand et al., 2006; Cárdenas et al., 2008a,b; Lee, 2006; Mo et al., 2011; Mueller, 2006; Sun et al., 2009). Each of these specific characteristics potentially opens up new threats to CPSs that do not exist in conventional IT systems. The study of security in CPSs can hence be divided into different categories based on the specific characteristics that are addressed, such as: stringent resource constraints, timing behavior under shared resources, etc.

The stringent resource constraints in CPSs are an important barrier to the implementation of robust security measures (Aminifar et al., 2017; Hwang et al., 2006; Jiang et al., 2011, 2013; Kang and Son, 2006; Lin et al., 2009). The reduced computational power of underlying micro-controllers might strongly limit the ability of CPSs to perform security tasks, e.g., cryptographic operations (Kocher et al., 2004; Ravi et al., 2004; Sagstetter et al., 2013; Sun et al., 2009).

The shift towards integrated architectures in CPSs (Di Natale and Vincentelli, 2010) has resulted in sharing of resources between different control systems. The implementation of control applications on shared platforms consists of two main steps: controller synthesis and control task scheduling. The traditional design flow of such applications is based on the principle of separation of concerns. The main drawbacks of this principle are poor resource utilization and control performance (Årzén and Cervin, 2005). This is due to the fact that the effects of the decisions made during one of the design steps are not considered on the decisions that are made during the other steps, which often leads to suboptimal solutions. As a result, the control scheduling co-design methodology is proposed to optimize control performance while considering scheduling constraints. The scheduling problem associated with shared resources and real-time demands has been addressed previously (Aminifar, 2016; Avni et al., 2016; Chakraborty et al., 2002; Craciunas et al., 2016; Pop et al., 2003, 2013; Samii et al., 2009). However, the control scheduling co-design may impact the temporal behavior of the system. The impact of temporal properties on control performance and stability of CPSs has been studied previously (Aminifar et al., 2015b, 2018; Cervin, 2012; Cervin et al., 2019; Deng et al., 2016; Goswami et al., 2012; Lincoln and Cervin, 2002; Quaglia et al., 2012; Samii et al., 2009; Wittenmark et al., 1995), both in the case of net- 
worked CPSs (Deng et al., 2016; Goswami et al., 2012; Quaglia et al., 2012; Samii et al., 2009) and CPSs with uniprocessor platforms (Aminifar et al., 2015b, 2018). However, none of the previous works address the security challenges related to the temporal aspects and, implicitly, the schedule synthesis of CPSs.

The timing behavior of CPSs not only impacts their control performance, but also make them vulnerable to security threats. Mueller (Mueller, 2006) indicates that lack of proper verification and validation of timing constraints may result in system malfunction. In (Sun et al., 2009) the vulnerabilities that result from contradictory requirements between real-time constraints and security needs are highlighted. Moreover, the control scheduling co-design and the necessary optimization for the constrained resources may expose the system to abnormal timing behavior. This design sophistication, on one hand, makes the implementation of security measures more complicated, and on the other hand, exposes the CPSs to new types of attacks (Shoukry et al., 2013a).

In another line of work (Amin et al., 2009, 2010; Cárdenas et al., 2008a,b; Ren et al., 2020; Shoukry et al., 2013a), the effort is to utilize control theory to detect attacks and design control systems that are robust to them. In (Liu et al., 2015; Mo and Sinopoli, 2014; Pajic et al., 2016; Ren et al., 2020; Sandberg et al., 2010), the authors use state estimations in CPSs with multiple sensors to deal with inputs that have been manipulated by attackers. Shoukry et al. (Shoukry et al., 2013a) consider an attacker that is trying to destabilize a control system by changing the order in which packets are delivered to the controller. Amin et al. (Amin et al., 2009) consider the case where the control packets may be jammed by a malicious adversary. Both Amin et al. and Shoukry et al. propose redesigning the controller so that it can tolerate jamming or reordering of packets. The aforementioned efforts use control theory techniques to detect attacks and make the control application robust. Therefore, the proposed methods require the controller to be redesigned. In Chapter 3 and Chapter 5 we provide security solutions that do not require redesigning the controller and are integrated during the implementation of the control application. 
When it comes to defense strategies to address security threats to CPSs, the approaches can be classified as prevention, detection, and mitigation techniques (Lun et al., 2019).

Preventing attacks has been addressed using conventional IT security approaches like firewalls (Okhravi and Sheldon, 2010). Another prevention approach is to implement cryptographic protections (Roman et al., 2011). In the context of this thesis, we highlight the cryptographic approaches that are specifically designed to satisfy the resource constraints and timing demands of CPSs. Jasmin and Hemalatha (Jasmin and Hemalatha, 2017) introduce an encrypting/decrypting module that is suitable for control systems with limited computational capabilities. Kogiso and Fujita (Kogiso and Fujita, 2015) exploit a modified homomorphic encryption scheme to secure networked control systems. They provide a lightweight cryptographic algorithm and discuss the performance degradation of the networked control system due to encryption/decryption. In Chapter 5 we discuss more on implementing cryptographic protocols in a stringent resource-constrained CPS.

Detecting attacks is conventionally done by implementing intrusion detection mechanisms in IT systems (Garcia-Teodoro et al., 2009). In the context of CPSs, anomaly detection can be done by observing the internal states (Zimmer et al., 2010). In (Amin et al., 2010; Sajjad et al., 2015; Wei and Kundur, 2015), the authors exploit the extra information from the underlying CPS to propose more accurate application-specific anomaly detection algorithms. In the context of anomaly detection approaches, a major advantage of control systems compared to classical IT systems is the implicit availability of the model of the physical world. This makes it possible to use the model of the physical plants to reduce false positives in the detection algorithms. Physicsbased attack detection techniques are studied in (Giraldo et al., 2018). The use of the model of the physical plant to detect anomalies is proposed in (Aoudi et al., 2018; Cárdenas et al., 2011; Cheung et al., 2007; Sridhar and Govindarasu, 2014). Zimmer et al. (Zimmer et al., 2010) use the information on the worst-case execution time bounds and detect anomalies by comparing the timing metrics to the actual execution. Yoon et al. (Yoon et al., 2013) detect malicious activities in real-time systems by statistical analysis of the execution profiles. In Chapter 3, we discuss more on utilizing the physical model of a plant to detect anomalies in control applications. 
Mitigating malfunction in CPSs has been studied for a long time in the context of safety and reliability in order to protect a system against hazards and random faults (Izosimov et al., 2012; Jiang, 2005; Noura et al., 2009; Samii et al., 2011; Yin et al., 2013). In (Fawzi et al., 2014; Mo and Sinopoli, 2014), the authors propose a resilient state estimation technique to mitigate the impact of an attack that comprises a subset of sensors. Authors in (Cárdenas et al., 2011) mitigate the attack by replacing the sensed measurements with their estimate. Another sort of mitigation mechanism that is inspired by the fault-tolerant control approaches is to reconfigure the controller and devise a reactive strategy to compensate for the damage from the attack. This can be done, for example, by changing to a reactive control signal (Barreto et al., 2013), transmitting signals with a higher power when a jamming attack is detected (Liu et al., 2014), or by increasing the sampling frequency of the system (Farraj et al., 2014; Mahfouzi et al., 2016). In these cases, attack mitigation is protecting CPSs from faults that are caused intentionally by adversaries. In Chapter 3, we elaborate more on how resource management can be used to mitigate attacks on control applications.

Recently, CPSs are also following the trend towards outsourcing computation to the cloud (Mubeen et al., 2017; Skarin et al., 2020). One of the challenges when the controller is outsourced is security. The authors of (Alexandru et al., 2018; Farokhi et al., 2017; Kim et al., 2016; Kogiso and Fujita, 2015) use homomorphic encryption to address the confidentiality of messages to/from the outsourced controller. In (Jutla, 2001), the authentication and integrity issues in the communication channel are discussed. In the context of CPSs, none of the current literature addresses the study of security measures in a context in which the computational results provided by the cloud have already been manipulated. We investigate this problem in Chapter 6 .

\subsection{Summary of Contributions}

In this thesis, we focus on security measures for control systems. We highlight the specific characteristics of such systems and use the knowledge from the physical model of the plant to detect and mitigate attacks against CPSs (Mahfouzi et al., 2016). We emphasize the complex timing behaviors of CPSs that result from using shared resources. Furthermore, we introduce attack scenar- 
ios that exploit the non-intuitive timing characteristics of control systems to render them unstable (Mahfouzi et al., 2019b). We also study the problem of synthesizing routes and schedules for networked CPSs using time-triggered Ethernet switches. We provide an integrated routing and scheduling framework using Satisfiability Modulo Theory and heuristic approaches (Mahfouzi et al., 2018). On top of that framework, we highlight security issues and investigate the impact of security measures on the stability of control applications. We use design space exploration approaches to maximize the security level while guaranteeing control stability (Mahfouzi et al., 2019a). Finally, the security challenges with regard to outsourcing a time sensitive control application to the cloud are discussed. A cryptographic approach to verify the correctness of the results received from the cloud is studied and a proof of concept for cloud-based implementation of a control application with verifiable computation is provided (Mahfouzi et al., 2021). In the following subsections, we summarize the main contributions of this thesis.

\subsubsection{Intrusion Detection and Mitigation for Control Applications Under Observable and Controllable Attacks}

Dealing with intrusions is one of the most important aspects of securing a CPS. In Chapter 3 we consider the class of security measures that deals with new, unanticipated, unaccounted for, or zero-day attacks, as opposed to security measures that are implemented based on known attack strategies (e.g., signature-based detection and prevention mechanisms). In realistic scenarios, the designer cannot cover all attack scenarios at design time. Therefore, in addition to the static security measures deployed at design time, there is a need for online, flexible security measures.

In Chapter 3, we highlight several important properties of control applications in CPSs. We suggest a systematic intrusion-damage assessment and mitigation framework for the class of observable and controllable attacks based on these properties. On the one hand, plants in CPSs adhere to certain laws of physics, which can be used to solve the intrusion-damage assessment issue. That is, the states of the controlled plant should match those predicted by the system's physics, and any significant deviation may indicate an in- 
trusion. On the other hand, the amount of allocated resources has a strong impact on control efficiency, which can be used to resolve the intrusion-damage mitigation issue. That is, the intrusion-damage mitigation is performed by allocating more resources to the control application that is under attack. We use a machine learning algorithm to capture the system's normal behavior based on its dynamics. To minimize the damage caused by the intruder, we perform resource reallocation using a feedback-based approach with a convex optimization.

\subsubsection{Temporal Attacks on Control Applications}

To reduce costs, in CPSs, several control applications are often deployed on a shared platform, where both high and low criticality tasks run together. In Chapter 4 we highlight the complex timing behavior that results from such resource sharing. We show how resource management on a shared platform might result in counter-intuitive timing anomalies. We show how, by exploiting this feature, adversaries can destabilize a critical control system, which can result in irreversible consequences. We call this new attack scenario on CPSs a Butterfly attack. In a Butterfly attack, the adversary interferes with a lower criticality, less protected task that is running on a shared platform alongside the target task. The interference indirectly results in instability in the target task, which is potentially more critical. In Chapter 4, we also showcase the possibility of Butterfly attacks using two case studies from the automotive and avionic domains.

\subsubsection{Security and Stability-Aware Routing and Scheduling of Ethernet TSN Messages}

In Chapter 5, we focus on the underlying network as a shared communication channel inside a CPS. Real-time communication is predominantly shifting towards using Ethernet especially in applications such as industrial automation and control, avionics, and automotive networking. Since time criticality is an inherent characteristic of these CPSs, the IEEE 802.1 TSN standards are developed to enhance Ethernet technology to support time-triggered communication. In Chapter 5 we highlight the strong impact of network communication timing on the performance and stability of control applications. We 
solve the joint routing and scheduling problem in an Ethernet TSN network while explicitly taking into consideration the impact of communication delay and jitter on control quality. A heuristic approach is then proposed in Chapter 5 to circumvent the inherent complexity of the joint route and schedule synthesis problem.

Furthermore, in Chapter 5 we also propose a security-aware methodology that is intended to be implemented on top of the integrated routing and scheduling. The goal is to perform routing and scheduling so that the security level provided by encryption and decryption is maximized while keeping the end-to-end delay sufficiently low so that the stability of the control system is guaranteed.

\subsubsection{Secure Cloud-Based Control Using Verifiable Computation}

In Chapter 6 we consider the scenario of outsourcing a control application to the cloud and the security challenges implied by such an approach. We highlight the necessity of a mechanism for verifying the control signal received from the outsourced controller running in the cloud. We employ a recent cryptographic technique, called verifiable computation, that allow a client to check the correctness of a remote execution. In Chapter 6 we deliver a proof of concept implementation to show the applicability of verifiable computation techniques for control applications. We specifically show the practicality of verifiable computation schemes with advanced control methods like Model Predictive Control (MPC), wherein the computationally heavy part of the controller is outsourced to a cloud platform.

\subsection{List of Publications}

Following are the peer-reviewed articles related to the contributions included in this thesis:

- Rouhollah Mahfouzi, Amir Aminifar, Petru Eles, Zebo Peng, and Mattias Villani. "Intrusion-damage assessment and mitigation in cyberphysical systems for control applications." In Proceedings of the 24th 
International Conference on Real-Time Networks and Systems, pp. 141150. (Mahfouzi et al., 2016).

- Rouhollah Mahfouzi, Amir Aminifar, Soheil Samii, Ahmed Rezine, Petru Eles, and Zebo Peng. "Stability-aware integrated routing and scheduling for control applications in Ethernet networks." In 2018 Design, Automation \& Test in Europe Conference \& Exhibition (DATE), pp. 682-687. (Mahfouzi et al., 2018).

- Rouhollah Mahfouzi, Amir Aminifar, Soheil Samii, Petru Eles, and Zebo Peng. "Security-aware routing and scheduling for control applications on Ethernet TSN networks." ACM Transactions on Design Automation of Electronic Systems (TODAES) 25.1, pp. 1-26. (Mahfouzi et al., 2019a).

- Rouhollah Mahfouzi, Amir Aminifar, Soheil Samii, Mathias Payer, Petru Eles, and Zebo Peng. "Butterfly Attack: Adversarial Manipulation of Temporal Properties of Cyber-Physical Systems." In 2019 IEEE Real-Time Systems Symposium (RTSS), pp. 93-106. (Mahfouzi et al., 2019b).

- Anton Cervin, Paolo Pazzaglia, Mohammadreza Barzegaran, and Rouhollah Mahfouzi. "Using jittertime to analyze transient performance in adaptive and reconfigurable control systems." In 2019 24th IEEE International Conference on Emerging Technologies and Factory Automation (ETFA), pp. 1025-1032. (Cervin et al., 2019).

- Rouhollah Mahfouzi, Amir Aminifar, Soheil Samii, Ahmed Rezine, Petru Eles, and Zebo Peng. "Breaking Silos to Guarantee Control Stability with Communication over Ethernet TSN." IEEE Design \& Test (Mahfouzi et al., 2020).

- Rouhollah Mahfouzi, Amir Aminifar, Soheil Samii, Petru Eles, and Zebo Peng. "Secure Cloud Control Using Verifiable Computation" IEEE International Conference on Omni-layer Intelligent systems (COINS) pp. 93-106. (Mahfouzi et al., 2021). 


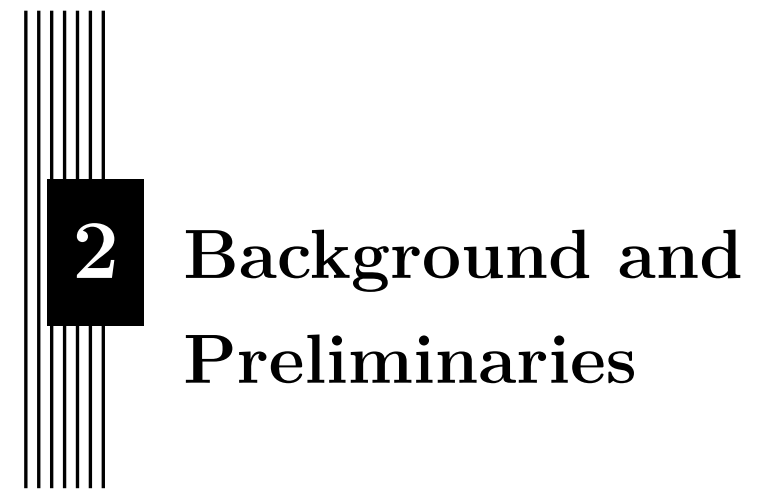

In this chapter, we introduce the basic notions underlying the discussion in the rest of the thesis. We introduce an overview of cyber-physical systems and some of the control theory behind them. Furthermore, in the context of integrated architectures the notion of a shared platform is introduced comprising both the computation and the communication infrastructure. We then introduce the problem of task/message scheduling on the shared platform and the connection between schedule synthesis and the timing characteristics of the controller derived from control laws. Then, the security threats we consider in this thesis are discussed, followed by an illustration of the fundamental cryptographic mechanisms for maintaining confidentiality and authentication. Finally, some common assumptions regarding outsourcing a controller to the cloud are described and the Model Predictive Control (MPC) method is explained. 


\subsection{System Model}

In many CPSs, multiple control applications use a shared resource. Let us consider a system comprised of a set of $n$ control applications $\boldsymbol{\Lambda}$. Each control application $\Lambda_{i} \in \boldsymbol{\Lambda}$ consists of a physical plant $P_{i} \in \mathbb{P}$, a sensor $S_{i}$, and a controller $C_{i} \in \mathbb{C}^{1}$. The control application $\Lambda_{i}$ is periodic and the sampling period is denoted by $h_{i}$. The controller $C_{i}$ is responsible for keeping the physical plant $P_{i}$ stable. The controller $C_{i}$ is implemented as control task $\tau_{i} \in \mathbb{T}$. The set of all control tasks is denoted by $\mathbb{T}$.

The shared resource among these $n$ control applications can be a processing unit that is shared between several controller tasks or a communication network that is shared among several control applications to transmit their messages. In the following we describe these two scenarios.

\subsubsection{Shared Processor}

In Figure 2.1, the scenario in which several controller tasks are run on the same processing unit is illustrated. The sampling period of control task $\tau_{i}$ is denoted by $h_{i}$. In each sampling period, the controller $C_{i}$ reads the state of the physical plant $P_{i}$ from sensor $S_{i}$ attached to physical plant $P_{i}$. Then the control task $\tau_{i}$ executes and calculates the proper output. The calculated output is then sent back to the physical plant to be actuated. Each instantiation of task $\tau_{i}$ has a different execution time on the processing unit, depending on the input to the task. The execution time of $\tau_{i}$ at the $j$-th instantiation of the task is denoted by $c_{i}^{j}$. Therefore, task $\tau_{i}$ has the following parameters:

- the worst-case execution time is denoted by $c_{i}^{\mathrm{w}}$;

- the best-case execution time is denoted by $c_{i}^{\mathrm{b}}$;

- the sampling period is denoted by $h_{i}$.

There might be other tasks on the processing unit that are not control tasks and are not necessarily periodic, such as, event-based tasks. The well-studied

\footnotetext{
${ }^{1}$ We are considering this one-to-one sensor-controller correspondence only for the discussion in the thesis, in order to keep the notation and equations readable. Our implementation in the next chapters does not impose this restriction.
} 


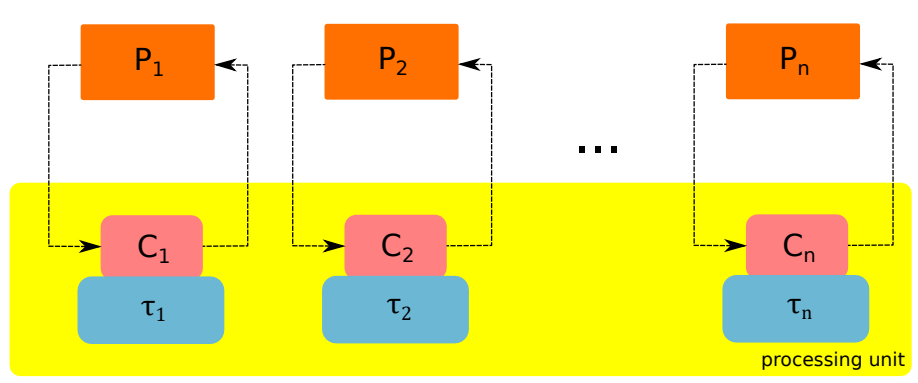

Figure 2.1: Control applications sharing a processing unit.

task scheduling problem is to determine a proper schedule for all the tasks running on the shared processor so that they all meet their deadlines.

\subsubsection{Shared Network}

In many networked CPSs a communication channel is used to transmit messages between sensors and controllers. In many applications, including the automotive industry and avionics, various communication technologies are used to transmit control messages. Examples are CAN, FlexRay, LIN, and Ethernet. In this thesis, we consider Ethernet which is becoming the predominant network technology in many application areas. We consider a network of Ethernet switches that is used as a shared platform to connect sensors and controllers of $n$ control applications. An example topology is depicted in Figure 2.2.

Each sensor $S_{i}$ periodically samples the state of a physical plant and sends it to a controller $C_{i}$. This data is used by the controller to calculate the proper output to be actuated on the physical plant. A series of message instances released in successive sampling periods of a control application is called a flow. Flow $f_{i}$ originates from sensor $S_{i}$ and is sent to controller $C_{i}$. The $j$-th message in this flow, which is sent at the $j$-th sampling period from sensor $S_{i}$ to controller $C_{i}$ is denoted by $m_{i}^{j}$. Since we know the exact period of each control application, in a hyper-period, a finite number of messages are transmitted via the shared network. The set of all control messages transmitted over a shared network in a hyper-period is denoted by $\mathscr{M}$. 


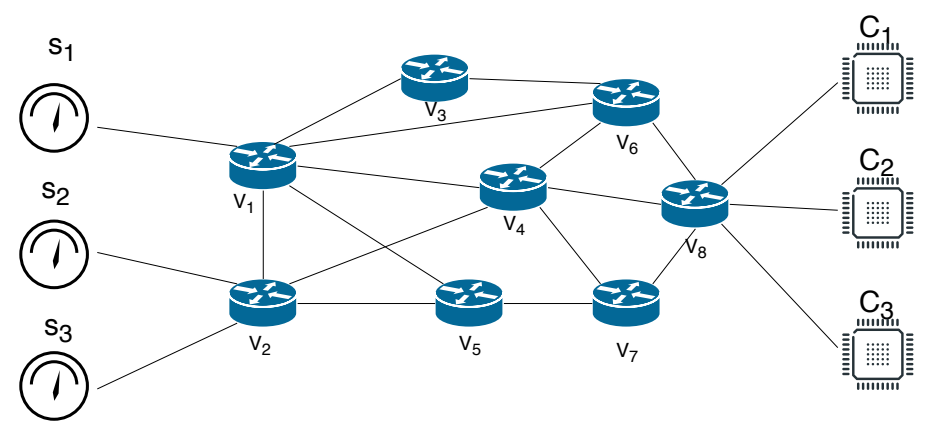

Figure 2.2: Control applications sharing a communication network.

In the case of safety-critical control applications with hard-deadlines, static routing and scheduling of all messages is preferably employed. Observe that the problem of finding routes and schedules over a shared network is at its roots similar to the problem of scheduling control tasks on a shared processor. The Ethernet switches used in this case are developed based on the IEEE 802.1 Time-Sensitive Networking (TSN) standard (IEEE Standard, 2015). The main characteristics of these switches are described below.

\subsubsection{Ethernet TSN Switches}

An overview of an Ethernet TSN switch with 4 ports is shown in Figure 2.3. In the figure, only the ingress portions of ports $\mathrm{A}$ and $\mathrm{B}$ and the egress portions of ports $\mathrm{C}$ and $\mathrm{D}$ are shown. All ports have a similar ingress composition to ports $\mathrm{A}$ and $\mathrm{B}$ and a similar egress composition to ports $\mathrm{C}$ and D. Based on the Ethernet TSN standard, in each output port up to 8 queues are dedicated for time-triggered (TT) messages. Other queues with lower priorities, i.e., Rate-Constraint (RC) and Best-Effort (BE), are used for less time-sensitive traffic. The incoming messages first go through a Forwarding Engine. In the Forwarding Engine, the output port is determined and the message is forwarded to the proper Dispatcher. The dispatcher assigns the message to a queue according to the priority code point $(\mathrm{PCP})$ of the header embedded in an IEEE 802.1Q Ethernet frame. There is a timed-gate at the end of each queue for time-triggered (TT) messages. These timed-gates enable or disable the transmission of messages from that queue according to a predefined static schedule. If the timed-gates for multiple queues are open then the 


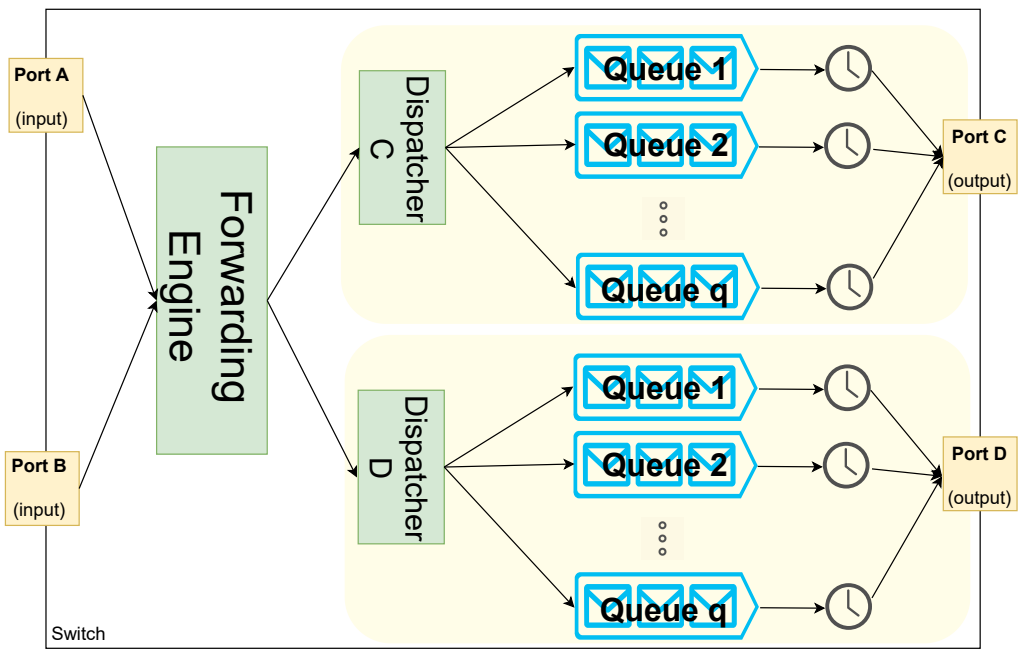

Figure 2.3: Logical overview of an Ethernet TSN switch with 4 ports. The ingress portions of ports $\mathrm{A}$ and $\mathrm{B}$, and the egress portions of ports $\mathrm{C}$ and $\mathrm{D}$ are shown.

message in the queue with the higher priority is transmitted. These timedgates facilitate the possibility of statically scheduling the release time of each message. Moreover, the route that the message should take is also determined in the table stored by the forwarding engine. Therefore, the output port and release time of a time-triggered message arriving at the switch can be stored in the switch. These two values are usually decided during route and schedule synthesis of messages.

\subsubsection{Plant Model}

The state-space model of a linear time-invariant plant $P_{i} \in \mathbb{P}$ is represented as (Åström and Wittenmark, 1997),

$$
\begin{aligned}
\dot{\boldsymbol{x}}_{i} & =\boldsymbol{A}_{i} \boldsymbol{x}_{i}+\boldsymbol{B}_{i} \boldsymbol{u}_{i}+\boldsymbol{v}_{i}, \\
\boldsymbol{y}_{i} & =\boldsymbol{C}_{i} \boldsymbol{x}_{i}+\boldsymbol{D}_{i} \boldsymbol{u}_{i}+\boldsymbol{e}_{i},
\end{aligned}
$$

where vectors $\boldsymbol{y}_{i} \in \mathbb{R}^{p}, \boldsymbol{x}_{i} \in \mathbb{R}^{n}$, and $\boldsymbol{u}_{i} \in \mathbb{R}^{m}$ are the plant output, the plant state, and the control signal, respectively. The first equation is the state equation and the second one is the output equation. The additive plant disturbance and the measurement noise are denoted by $\boldsymbol{v}_{i}$ and $\boldsymbol{e}_{i}$, respectively. 
$\boldsymbol{A}_{i} \in \mathbb{R}^{n \times n}$ is the state matrix and describes the underlying dynamics of the system and how internal states are connected to each other. $\boldsymbol{B}_{i} \in \mathbb{R}^{n \times m}$ is the input matrix and describes how plant inputs are entered into the system and which states they are affecting. $\boldsymbol{C}_{i} \in \mathbb{R}^{p \times n}$ is the output matrix and describes how the states are put together to get the output. $\boldsymbol{D}_{i} \in \mathbb{R}^{p \times m}$ is the feed-forward matrix and is used to bypass the plant and feed-forward the plant input to the plant output.

Note that the plant output $\boldsymbol{y}_{i}$ is a linear combination of the plant state $\boldsymbol{x}_{i}$ and the plant input $\boldsymbol{u}_{i}$. In many cases the plant output $\boldsymbol{y}_{i}$ is simply the state of the system, where $\boldsymbol{D}_{i}=0$ and $\boldsymbol{C}_{i}=\boldsymbol{I}$. In this case, the state space model is described only by the first equation in Equation 2.1 as follows,

$$
\dot{\boldsymbol{x}}_{i}=\boldsymbol{A}_{i} \boldsymbol{x}_{i}+\boldsymbol{B}_{i} \boldsymbol{u}_{i}+\boldsymbol{v}_{i}
$$

\subsection{Control Performance}

In order to measure the performance of a control application $\Lambda_{i}$ in connection with physical plant $P_{i}$ we use a standard quadratic cost (Åström and Wittenmark, 1997):

$$
\lim _{T \rightarrow \infty} \frac{1}{T} \mathbb{E}\left\{\int_{0}^{T}\left[\begin{array}{l}
\boldsymbol{x}_{i} \\
\boldsymbol{u}_{i}
\end{array}\right]^{\top} \boldsymbol{Q}_{i}\left[\begin{array}{l}
\boldsymbol{x}_{i} \\
\boldsymbol{u}_{i}
\end{array}\right] d t\right\} .
$$

The weight matrix $\boldsymbol{Q}_{i}$ is a positive semi-definite matrix and is given by the designer. The matrix determines the relative impact of each element of the plant state or of the control input array on the final control cost. $\mathbb{E}\{$.$\} denotes$ the expected value of a stochastic variable.

Intuitively, the standard quadratic cost function captures the deviation of the state of the plant from the desired state and the amount of energy (control input) needed to stabilize the system. Therefore, a larger control cost indicates worse control performance. An infinite control cost indicates that the controller is unstable (Lincoln and Cervin, 2002).

Two main elements that contribute directly to the control performance are sampling period and delay between sensing and actuation. The sampling period determines how often the plant is being controlled by the controller 


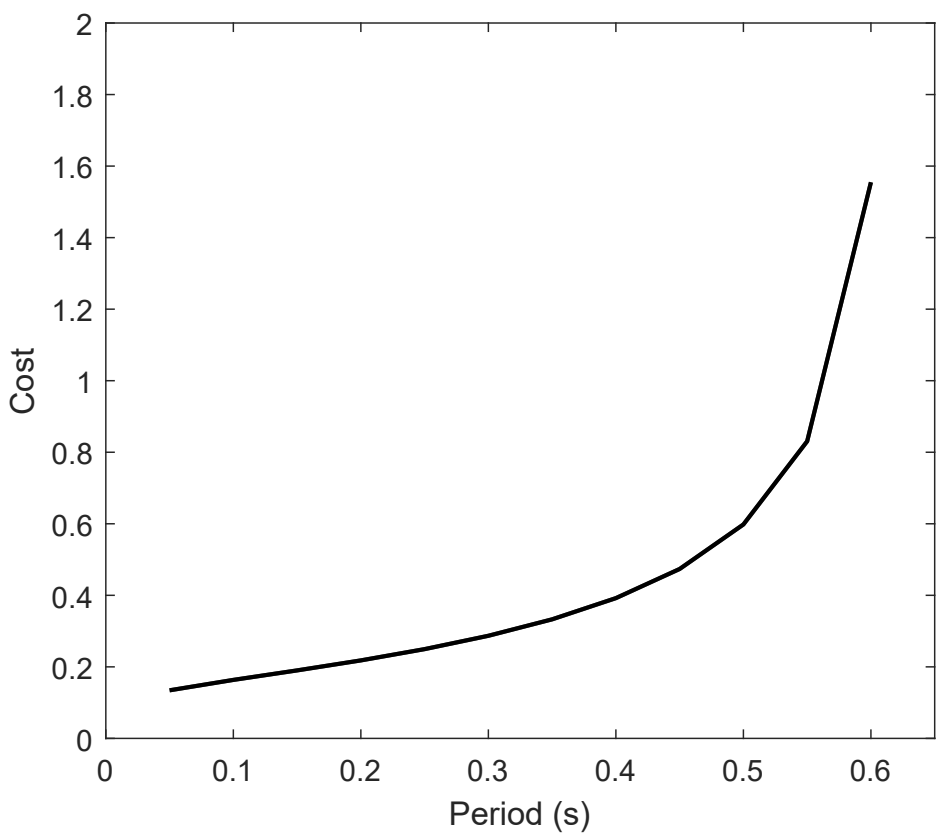

Figure 2.4: Control cost vs. the sampling period for a constant delay.

task. Intuitively, the more frequently the plant is controlled (smaller periods) the better the performance would be, hence leading to lower control cost. Figure 2.4 shows the control cost of an inverted pendulum for different periods in the interval of $[0.05-0.6]$ seconds. The figure demonstrates that for a constant delay, control cost increases (performance gets worse) as the sampling period increases.

In the ideal case for synthesizing the controller, the delay between sensing and actuation is considered to be zero. However, this is not possible in reality. The source of such a delay could be:

1. actual transmission delay of the message in the network,

2. delay due to queuing of the message in the switches of the network,

3. execution time of the task on the controller, and

4. delay due to interference from other tasks on the shared processor. 


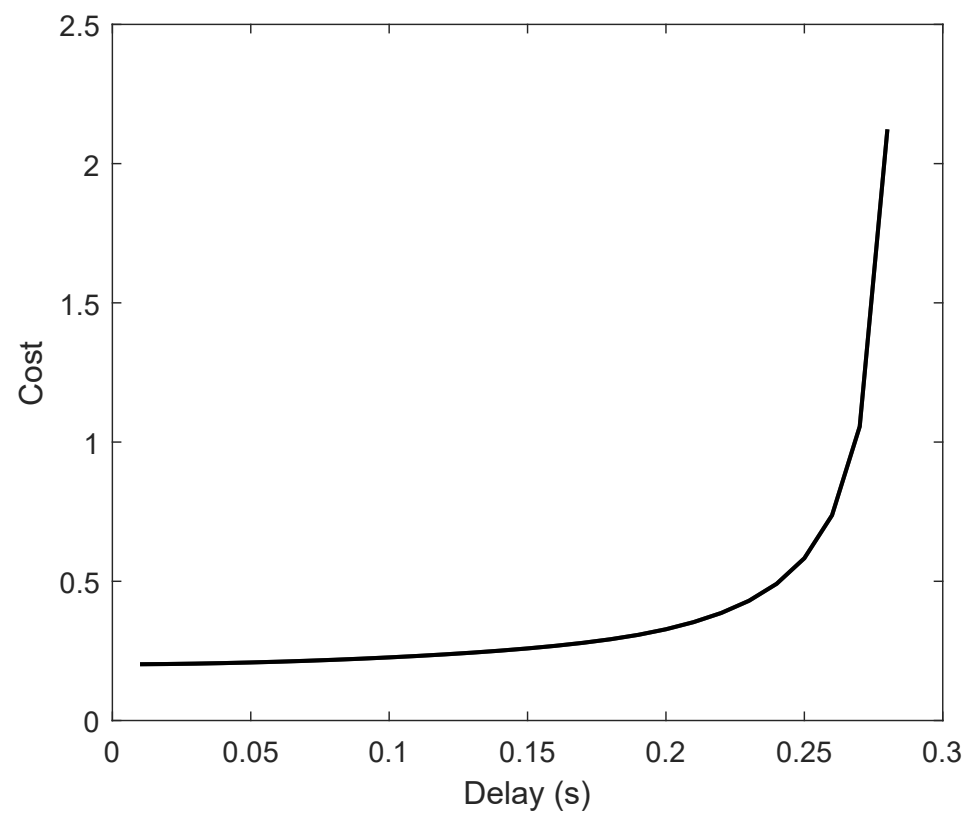

Figure 2.5: Control cost vs. the delay between sensing to actuation for a fixed sampling period, $h=0.2$.

Figure 2.5 considers an inverted pendulum and demonstrates how control cost increases (performance gets worse) as the delay increases between sensing and actuation.

In the experiments above the delay between sensing and actuation is assumed to be constant. However, this is usually not the case as the delay is variable due to varying message delay, varying execution time, and varying interference. In addition to the delay itself, the variation of the delay also contributes to the actual control performance. We discuss the effect of varying delays on control performance in Section 2.3.

\subsection{Stability Analysis}

Normally, the controller is designed for a constant delay between sensing and actuation. However, this delay varies in each sampling period of the controller 


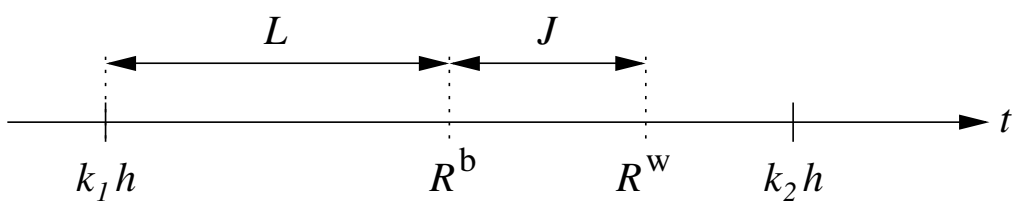

Figure 2.6: Graphical interpretation of the latency and worst-case jitter. Best-case and worst-case response time is denoted by $R^{\mathrm{b}}$ and $R^{\mathrm{w}}$ and $h$ is the sampling period of the controller.

due to the different execution times of the controller task, the interference on the shared platform, and/or different communication delays of control messages. The control performance degrades due to varying delays between sensing and actuation. Hence, during schedule synthesis, it is important to consider how tolerant the control application is to varying delays in order to guarantee the stability of the system.

For a control application $\Lambda_{i}$, the delay between sensing and actuation is called end-to-end response time (or end-to-end delay) and is denoted by $R_{i}$. The response time varies between different sampling periods. Maximum and minimum response time for a control application are called worst-case and best-case response-time and are denoted by $R_{i}^{\mathrm{w}}$ and $R_{i}^{\mathrm{b}}$, respectively. The calculation of worst-case and best-case response times has long been studied in the field of real-time systems and depends on the scheduling policy of tasks/messages, i.e., Earliest Deadline First (EDF), Fixed Priority Scheduling, etc.

Considering a control application $\Lambda_{i}$ the best-case response time $\left(R_{i}^{\mathrm{b}}\right)$ which is the constant part of the delay experienced by the control application is defined as latency, $L_{i}$. Jitter is the varying part of the delay. Figure 2.6 shows a graphical interpretation of latency and worst-case jitter. We define the worst-case jitter (or simply jitter) as the difference between maximum delay (worst-case response time) $R_{i}^{\mathrm{w}}$, and minimum delay (best-case response time) $R_{i}^{\mathrm{b}}$,

$$
\begin{aligned}
L_{i} & =R_{i}^{\mathrm{b}}, \\
J_{i} & =R_{i}^{\mathrm{w}}-R_{i}^{\mathrm{b}} .
\end{aligned}
$$

In order to investigate the impact of jitter on control performance, the worstcase control performance of the control application is defined. The worst-case 


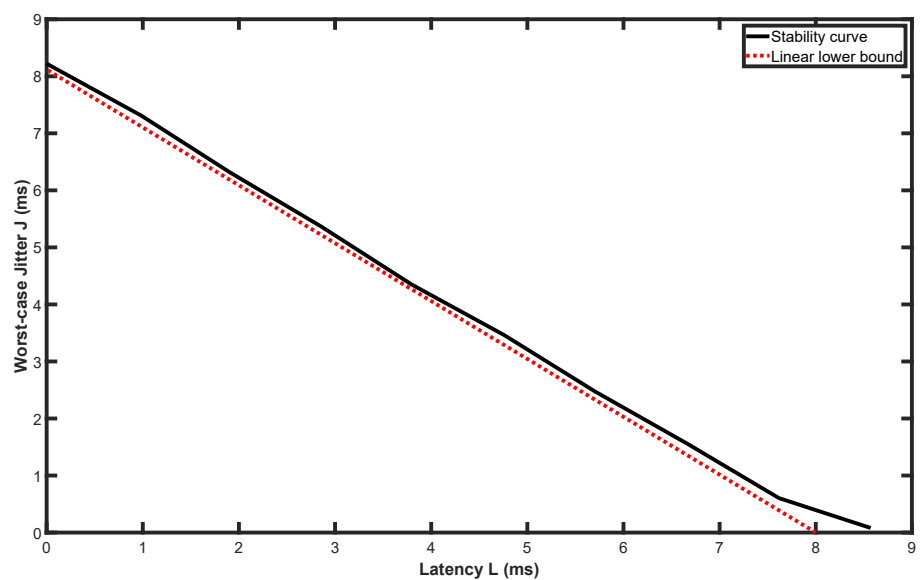

Figure 2.7: The stability curve generated by the Jitter Margin toolbox and the linear lower bound. The curve is generated for an inverted pendulum with length of $50 \mathrm{~cm}$ and a discrete-time Linear QuadraticGaussian (LQG) controller, with a sampling period of $8 \mathrm{~ms}$.

control performance can be quantified by an upper bound on the gain from time-varying delays. Basically, the worst-case control performance shows how tolerant the control application is toward varying delays. In order to measure the worst-case control performance, we use the Jitter Margin toolbox (Cervin, 2012). The notion of jitter margin (similar to phase margin and gain margin (Franklin et al., 2002)) for a periodic control loop is defined as a function of latency, and it describes how much additional time-varying delay (jitter) the plant can tolerate. Basically, the Jitter Margin shows, for a given latency, the maximum jitter under which the stability of the control loop is still guaranteed.

Figure 2.7 shows an example of the stability curve generated by the Jitter Margin toolbox. The curve is generated for an inverted pendulum with a length of $50 \mathrm{~cm}$ that is controlled by a discrete-time Linear Quadratic-Gaussian (LQG) controller, with a sampling period of $8 \mathrm{~ms}$. The area below the solid black line shows the stable area. The stability curve can be approximated by a linear function (see the red dotted line in Figure 2.7). Therefore, the stability condition for control application $\Lambda_{i}$ can be formulated as follows,

$$
L_{i}+\alpha_{i} \cdot J_{i} \leqslant \beta_{i} .
$$


In Equation 2.5, $\alpha_{i} \geqslant 1$ and $\beta_{i} \geqslant 0$ are constant coefficients that depend on the control application. ${ }^{2}$

By identifying the coefficients in Equation 2.5 for a given control application, one can determine the stability of that control application with respect to certain latency and jitter.

\subsection{Model Predictive Control}

Model Predictive Control (MPC) is an advanced control methodology and is often used in constrained Multiple Input Multiple Output (MIMO) systems. The MPC approach is used in a wide range of applications including automotive, avionics, and industrial processes. It employs the concept of the receding horizon, as shown in Figure 2.8. The figure shows the idea of MPC at sampling time $k$. The reference trajectory is indicated by $\boldsymbol{r}(k)$, which shows the ideal trajectory for the controlled output, $\boldsymbol{y}(k)$. At time $k$ the solid part of the controlled output trajectory shows the past states and the dotted curve indicates the predicted states. $\boldsymbol{u}(k)$ denotes the control signal; the solid part captures the previous control signals applied to the system and the dotted curve shows the calculated control signals for the future. Basically, the dotted control signal, $\hat{\boldsymbol{u}}(k)$, is the optimal control signal which promises the best predicted control behaviour, depicted by the dotted curve, $\hat{\boldsymbol{y}}(k)$.

The MPC uses the plant model to determine the optimal control signals for a finite prediction horizon, $\boldsymbol{H}_{\boldsymbol{p}}$. Basically, at each sampling time $k$, the optimal control signals, $\hat{\boldsymbol{u}}(k+i \mid k), i=0,1, \ldots, \boldsymbol{H}_{\boldsymbol{p}}-1$, that predict the best control output (closest to the reference trajectory) are calculated. The first calculated control signal is actuated on the physical plant and the rest are discarded. At the next sampling time, the prediction horizon remains the same size as before but slides along by one step, hence this controlling method is called receding prediction horizon.

\footnotetext{
${ }^{2}$ Here we describe the simple case of linear stability condition for simplifying the discussion. In practice, a piecewise linear approximation can be used in order to achieve better accuracy. In that case, multiple equations with different $\alpha$ and $\beta$ coefficients will formulate the stability condition.
} 


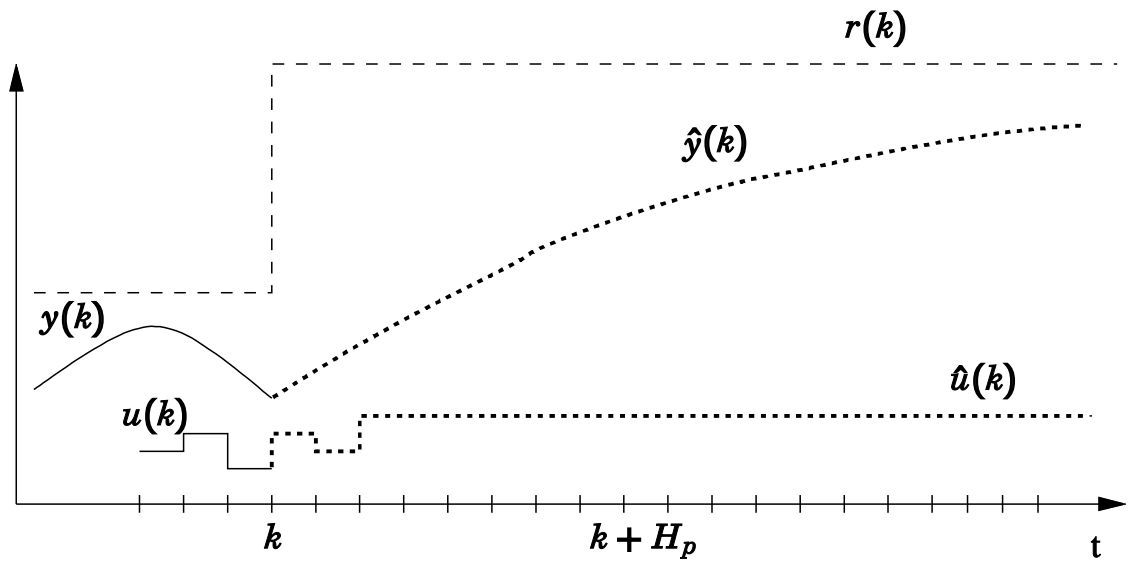

Figure 2.8: Basic illustration of predictive horizon. $r(k)$ is the reference trajectory, $y(k)$ is the controlled output and $u(k)$ represents the control signal.

Consider the following linear discrete-time state-space model,

$$
\begin{aligned}
\boldsymbol{x}(k+1) & =\boldsymbol{A} \boldsymbol{x}(k)+\boldsymbol{B} \boldsymbol{u}(k), \\
\boldsymbol{y}(k) & =\boldsymbol{C} \boldsymbol{x}(k)+\boldsymbol{D} \boldsymbol{u}(k),
\end{aligned}
$$

where $\boldsymbol{x}$ is the state vector. The MPC controller should also satisfy the following constraints,

$$
\begin{gathered}
\Delta \boldsymbol{u}_{\min } \leqslant \Delta \boldsymbol{u}(k) \leqslant \Delta \boldsymbol{u}_{\max }, \\
\boldsymbol{u}_{\min } \leqslant \boldsymbol{u}(k) \leqslant \boldsymbol{u}_{\max }, \\
\boldsymbol{y}_{\min } \leqslant \boldsymbol{y}(k) \leqslant \boldsymbol{y}_{\max },
\end{gathered}
$$

where $\Delta \boldsymbol{u}(k)=\boldsymbol{u}(k)-\boldsymbol{u}(k-1)$ is the difference between control signals.

Now we can formulate the optimal control problem that is the basis of the MPC algorithm. The cost function for reducing the error between reference trajectory, $\boldsymbol{r}(k)$, and the predicted controlled output, $\hat{\boldsymbol{y}}(k)$, is as follows,

$$
J(k)=\sum_{i=0}^{\boldsymbol{H}_{\boldsymbol{p}}-1}\|\hat{\boldsymbol{y}}(k+i \mid k)-\boldsymbol{r}(k+i \mid k)\|_{Q}^{2}+\|\Delta \hat{\boldsymbol{u}}(k+i \mid k)\|_{R}^{2}
$$

where $Q \geqslant 0$ and $R \geqslant 0$ are weighting matrices and are constant over the prediction horizon. The second term in the cost function penalizes the variation in the control signal. 
Therefore, the optimal control problem can be defined to minimize the cost function in Equation 2.8, while considering the constraints in Equation 2.7.

It can be shown that the above optimal control problem with constraints can be rewritten in a standard optimization problem format known as Quadratic Programming (Maciejowski, 2002),

$$
\begin{array}{ll}
\min _{\theta} & \frac{1}{2} \theta^{T} \Phi \theta+\phi^{T} \theta \\
\text { s.t. } & \Omega \theta \leqslant \omega
\end{array}
$$

where $\theta$ is the variable matrix derived from the variables in Equations 2.7 and 2.8. $\Phi$ (positive semi-definite), $\phi, \Omega$, and $\omega$ are the coefficient matrices. There are many standard solutions for solving the quadratic programming problem. However, all of these solutions are computationally demanding. This makes the MPC controller complex and time-consuming especially when there is a limited processing power available for the controller. It is worth noting that, the control performance of an MPC controller improves when we consider larger prediction horizons. However, increasing prediction horizon $\left(\boldsymbol{H}_{\boldsymbol{p}}\right)$ also increases the complexity of the quadratic programming problem.

\subsection{Confidentiality and Integrity}

Confidentiality and integrity are among the fundamental objectives of information security (Stallings, 2013). Confidentiality means that data is protected from unauthorized viewing or access and it is provided using encryption. Integrity refers to protecting the data from unauthorized changes and is provided through authentication of the message and the sender. These two basic security services can be provided using a block cipher algorithm:

Confidentiality is provided if the sender and receiver use the same symmetric key to encrypt and decrypt the message. In this case, the sender encrypts the message using the symmetric key and the receiver decrypts it using the same key. Assuming that the encryption algorithm is secure, only the sender and receiver can access the unencrypted message (Stallings, 2013).

Integrity and Authentication can be provided using a Message Authentication Code (MAC). Given the message and symmetric key, the sender runs 
the block cipher algorithm to calculate a MAC. Then, the MAC is sent together with the message to the receiver. The receiver runs the same algorithm using the same key and the received message to re-calculate the MAC. If the received and the re-calculated MAC are identical then the integrity of the message is ensured. Moreover, the sender is also authenticated using this method, assuming that only the sender and the receiver have access to the symmetric key (Stallings, 2013).

\subsubsection{Authenticated Encryption}

A block cipher algorithm can simultaneously provide confidentiality, integrity, and authenticity using one symmetric key. In this case, the encryption/decryption algorithm will not only return the ciphertext/plaintext but will also create/verify the MAC.

Block cipher modes of operation: For a specific block cipher (e.g., AES and DEC) a mode of operation is a procedural rule integrated into the block cipher algorithm that results in different properties which adds to the security of the underlying block cipher. Example modes of operation are: Electronic Code Book (ECB), Cipher Block Chaining (CBC), Cipher block Chaining Message authentication code (CCM), Galois/Counter Mode (GCM), etc.

There are different approaches for implementing authenticated encryption. The U.S. National Institute of Standards and Technology (NIST) has standardized several modes of operation, e.g., CCM and GCM (Dworkin, 2003; Dworkin, 2007) for this matter. The block ciphers operating in these modes of operations simultaneously provide confidentiality and authentication. The authenticated encryption algorithms using these modes of operation have been used in many protocols e.g., IPsec, SSH, and SSL/TLS.

Using the GCM operation mode, authenticated encryption is provided with almost no extra cost (Jutla, 2001; Käsper and Schwabe, 2009) beyond that of the regular block cipher algorithm. In Chapter 5 we show how this approach is used to provide confidentiality and integrity of messages in a networked CPS. 


\section{Intrusion Detection and Mitigation for Control Applications Under Observable and Controllable Attacks}

In a control system, the states of the controlled plant should, in principle, correspond to what is expected according to the physics of the system and any major discrepancy is potentially an indication of intrusion. In this chapter, we use a machine learning algorithm to capture the normal behavior of the system according to its dynamics. Moreover, the control performance depends strongly on the amount of allocated resources, which can be used to mitigate the intrusion. The idea is to allocate more resources to the control applications that are under attack in order to mitigate any damage caused by the intrusion. This is done using a feedback-based approach including a convex optimization. In this chapter, our aim is to answer research question RQ1 (see Section 1.2). We propose a systematic intrusion-damage assessment and mitigation mechanism for the class of observable and controllable attacks. The content of this chapter is based on the following publication: (Mahfouzi et al., 2016). 


\subsection{Introduction and Related Work}

Due to growing connectivity of cyber-physical systems with the outside world, they are more exposed to attacks. One concrete example of attacks in CPSs is the non-invasive attack on anti-lock braking systems (ABS) in vehicles (Shoukry et al., 2013b), wherein an attacker can inject arbitrary speed measurements into the anti-lock braking system by exploiting weaknesses in the wheel speed sensors. There are many other examples of security threats and vulnerabilities in the case of in-vehicle communication networks (Hoppe et al., 2008; Lemke et al., 2006; Sagstetter et al., 2013), which demonstrates that ignoring the security aspects in CPSs can have severe consequences.

The StuxNet (Farwell and Rohozinski, 2011) attack worked by manipulating data sent from a sensor, indicating normal operation. At the same time, the control input data fed to the actuators was manipulated in a specific way to damage the physical plant. These examples highlight the security challenges in the area of CPSs and underscore the need for intrusion-damage assessment and mitigation techniques for such systems.

In general, there are two classes of security measures in CPSs:

- The first class is concerned with design time security measures (e.g., signature-based detection and prevention mechanisms), for all of the adversarial attacks that the designer can anticipate at design time. These approaches are often of the static type, where design time knowledge of the attack scenarios is required in order to develop countermeasures.

- The second class of security measures deals with new, unanticipated, unaccounted for, or zero-day attacks, since in realistic scenarios the designer cannot cover all attack scenarios at design time. Hence, there is a need for online and flexible (as opposed to static) security measures.

In this chapter, we focus only on the second class of security measures, and we assume that the first class of security measures are already in effect. Therefore, the major security challenge is to assess and mitigate the damage generated by unanticipated attacks. 
In Section 1.3 we indicated some related research contributions, in the following we include a more detailed discussion on related work. Compared to network intrusion detection, intrusion detection in the embedded systems area has gained less attention (Garcia-Teodoro et al., 2009). In (Zimmer et al., 2010), using offline information on the worst-case execution time bounds and by comparing the timing metrics to the actual execution, the authors can detect intrusions. In (Yoon et al., 2013), the authors aim to detect malicious activities by observing the inherent properties of real-time systems. However, both (Zimmer et al., 2010) and (Yoon et al., 2013) focus on real-time systems and require accurate knowledge of worst-case timing properties. Furthermore, neither of them addresses the intrusion mitigation challenge.

The idea of using a model for intrusion detection is not new (Denning, 1987). In the context of SCADA networks the authors of (Cheung et al., 2007) construct models that capture the acceptable behavior of such systems, and any deviation from these models indicates intrusion. However, the models and detection mechanism are limited to network communication protocols.

In (Sridhar and Govindarasu, 2014), the authors propose an intrusion detection and mitigation technique, designed for specific attack models against power systems. In particular, the mitigation technique is only valid for power systems and cannot be applied in a general setting.

Pasqualetti et al. discuss control-theoretic approaches to secure CPSs (Pasqualetti et al., 2015). They characterize fundamental attack detection and identification limits by modeling control systems and attackers. In (Cárdenas et al., 2008a), the authors discuss the fundamental difference between traditional embedded systems and CPSs and highlight the challenges in the design of secure CPSs. In particular, they highlight the need for intrusion response (mitigation) mechanisms.

In (Pasqualetti et al., 2013), the authors focus on attack detection in CPSs. However, no mitigation technique is suggested to counteract the adversary. In (Fawzi et al., 2011; Ivanov et al., 2014; Shoukry et al., 2015), the authors discuss estimation approaches to recover the state of the plant despite attacks on sensors. Therefore, the detection approach is restricted to sensor attacks. While this approach improves observability, it requires redundancy in hardware and, thus, additional costs. Moreover, such techniques are static in the 
sense that they only function up to a given upper bound on the number of attacked sensors, and beyond this they cannot dynamically overcome the attacker, as opposed to the technique proposed in this chapter, which can do so. In fact, the redundancy-based techniques can be applied in conjunction with the approach proposed in this chapter.

In (Cárdenas et al., 2011), the authors indicate that designing an automatic response mechanism to cyber attacks is one of the challenges in the area of secure cyber-physical systems. For the specific case of sensor attacks, the authors propose a simple response mechanism. Basically, they propose using the estimated state of the plant to compute the control input, in cases where the sensor data is being manipulated by an attacker. However, the mitigation mechanism is specific to sensor attacks and assumes the existence of an exact model. Furthermore, their approach, like many others, does not consider cases wherein several consecutive sensor readings are manipulated.

In (Fawzi et al., 2012), the authors discuss the possibility of making the system more resilient to attacks by an adequate choice of controller. That is, the controller can be designed to mitigate certain predetermined sensor attacks. The approach is, therefore, suitable for static scenarios, similar to the redundancy-based techniques, and cannot adapt to and mitigate new attack scenarios. That is, the approach belongs to the first class of the security measures discussed earlier in this section, and is therefore orthogonal to the proposed approach. Furthermore, similarly to (Cárdenas et al., 2011), the intrusion mitigation technique discussed is restricted to the specific case of sensor attacks.

In (Ghafouri et al., 2016), the authors find the optimal threshold, using an exhaustive search, for anomaly-based intrusion detection in dynamical environments, but this is done based on the strong assumption that the expected damage incurred by a system from an undetected attack at any instant in time is known. This is a common problem with binary intrusion detection approaches. That is, to find the optimal threshold to distinguish the intrusion state from the normal state, one needs to make unrealistic assumptions.

The idea of using advance machine learning techniques, like deep learning, for detecting anomalies in CPSs has emerged in the recent years (Ghafouri et al., 2018; Luo et al., 2021), mainly due to the increasing complexity of CPSs and 
more sophisticated attacks. However, these advanced machine learning techniques are computationally expensive and are mainly designed for CPSs with less timing constraints, hence not applicable in the context of this chapter.

\subsubsection{Important Properties of Control Applications}

In this subsection, we highlight certain properties of control applications in CPSs that can be used for systematic intrusion-damage assessment and mitigation.

Control applications in CPSs are distinct from other applications in the following ways:

- Control applications, by their nature, are often robust, in the sense that they are, to some extent, able to remain functional even in the presence of unexpected events. In the case that an attacker could destabilize a control system by tampering with the first message in the control loop, then there would be no sense in damage assessment and mitigation. For instance, for control applications, once a control command is dropped, the control application often tolerates this packet loss and remains stable, even though the packet loss degrades the control performance to some extent. Consequently, intrusion-damage assessment and mitigation are justified when it comes to control applications.

- In CPSs, the physical plants follow certain laws of physics derived from the dynamics of the plants. That is, the state samples of a plant over time cannot just be arbitrary and should follow certain physics-based laws. Often, a mathematical model of the physical plant is available, which can be used for intrusion-damage assessment.

- For control applications, the performance level is often strongly correlated with the amount of resources allocated to the control application (Aminifar et al., 2015a, 2012; Bini and Cervin, 2008; Cervin et al., 2004; Rehbinder and Sanfridson, 2000; Seto et al., 1996). This can be used for intrusion-damage mitigation. 


\subsubsection{Observable and Controllable Attacks}

Here we introduce the notion of observable and controllable attack. We focus on performance-observable and resource-controllable attacks. However, for the sake of brevity, we often drop performance and resource, and simply refer to these attacks as observable and controllable attacks.

In this chapter, we consider the class of observable and controllable attacks, inspired by the concepts of observability and controllability in control theory. We refer to an attack as observable and controllable if the performance degradation (i.e., the intrusion damage) caused by the attack can be observed and counteracted by the intrusion-damage assessment/mitigation unit. We elaborate more on the attack model in Section 3.3.

As long as the observability and controllability assumptions are met, the attack model in this chapter can be applied to any attack. For instance, the attack can be on either the sensor data delivered from the plant to the control task, or the control data sent to the plant by the control task. Similarly, the attacker may discard or manipulate the data in some sensor readings or actuator commands.

\subsubsection{Intrusion-Damage Assessment and Mitigation}

In this subsection, we propose an intrusion-damage assessment and mitigation technique for observable and controllable attacks.

\subsubsection{Intrusion-Damage Assessment}

As previously discussed, the plants in CPSs follow certain laws of physics, which is often captured by mathematical models. The availability of these mathematical models for physical plants can facilitate the intrusion-damage assessment process. In fact, using a model-based approach, any discrepancy between the expected state based on the model and the actual state of the plant is an indication of a potential attack. However, by definition, no model is exact and, therefore, we use a machine learning algorithm to capture the safe distance between the reality and the mathematical model in order to identify an attack. 
The only assumption for the proposed intrusion-damage assessment technique to work is that the effects of the attack should be observable by their impact on the performance of the control application. For example, if the attacker changes all the packets from all the sensors sent to the controller (or if all communication lines for the sensors are cut) such that we cannot observe the performance degradation, e.g., that an inverted pendulum is falling, then this attack is not observable and there is no systematic way to counteract the attack (Cárdenas et al., 2008a). However, if the attacker only changes some of the sensor values, then from the discrepancy between the expected sensor values and the sensor readings, we can detect the intrusion.

The key idea in this chapter is not only to consider the discrepancy between the expectation and the reality at each activation of the intrusion-damage assessment unit, but also to look into this discrepancy along several activations of this unit. This means that we consider the dynamic behavior of the system in a time window in order to assess intrusion. This aspect has been completely ignored in the previous research efforts. In addition, as opposed to the previous work in the literature, our intrusion-damage assessment unit does not report binary results ("no intrusion" versus "intrusion"), rather, we present the results using a continuous metric which captures the intrusion-generated damage. The larger the metric, the higher the damage. The continuous nature of the intrusion-generated damage metric is the main enabler of the intrusiondamage mitigation technique proposed in this chapter, as will be discussed in the following.

\subsubsection{Intrusion-Damage Mitigation}

The very next step after intrusion-damage assessment is to mitigate the effects of the attack. The key observation is that the control quality is related to the amount of resources dedicated to that control task (Aminifar, 2016; Cervin, 2003). Therefore, to counteract the attack, it is possible to allocate more bandwidth to the control application under attack. This increase in the allocated bandwidth should be commensurate with the damage generated by the intruder, i.e., intrusion-generated damage. Hence, the continuous nature of the intrusion-generated damage metric is essential for our mitigation technique to work. 
The only assumption for the proposed mitigation technique to work is (resource) controllability. That is, it is possible to counteract an intruder by assigning more processor and communication bandwidth to the control application under attack. For example, if the attacker discards all of the commands from the controller to the actuator, such that there is no way to control the plant, then this attack is not controllable and there is no systematic way to counteract the attack (Cárdenas et al., 2008a). However, if the attacker, for example, is only able to manipulate a few packets in a specific time window, then by assigning more bandwidth to this control task, e.g., by using a higher sampling rate, we can counteract the attacker.

An important advantage of our technique is its flexibility and generality when compared to static techniques. It is rather straightforward to dynamically increase the processing bandwidth for a control application in the case of an attack. And, it can be applied in any attack scenario, wherein the intrusiongenerated damage can be counteracted by increasing the processing bandwidth.

\subsection{System Model}

As discussed previously in Chapter 2 we consider a system that comprises $n$ plants $P_{i}$, each of which is controlled by a task $\tau_{i}$. In this chapter, we consider a uniprocessor platform shared among these tasks, as described in Section 2.1.1. However, the idea proposed in this chapter can be generalized to distributed systems with a shared network.

A plant $P_{i}$ is modeled by a continuous-time system of differential equations, as elaborated in Section 2.1.3. Note that, although we consider a linear model, the approach proposed in this chapter is by no means limited to linear models.

\subsubsection{Control Task Model}

The plant output $\boldsymbol{y}_{i}$ is sampled periodically and is used by the controller task $\tau_{i}$ to compute the control signal $\boldsymbol{u}_{i}$. Then, this control signal will be applied at the next sampling instant. The task $\tau_{i}$ parameters are:

- the worst-case execution time $c_{i}$; and 
- the sampling period $h_{i}$.

The sampling frequency of task $\tau_{i}$, denoted by $f_{i}$, is defined as follows,

$$
f_{i}=\frac{1}{h_{i}} \text {. }
$$

In order to measure the expected performance of a controller associated with plant $P_{i}$, we use a standard quadratic cost formulated by Equation 2.3.

Control applications can typically provide satisfactory control performance over a range of sampling periods. One extensively used rule of thumb for identifying the interval from which the sampling period, denoted by $h_{i}$, can be chosen is as follows (Åström and Wittenmark, 1997):

$$
0.2 \leqslant \omega_{i} h_{i} \leqslant 0.6
$$

where $\omega_{i}$ is the bandwidth of the closed-loop system of the corresponding control application. However, the above condition is only valid in the absence of any security intrusions.

In general, the higher the sampling rate is, the better the quality of control (Aminifar, 2016; Cervin, 2003). This has been also shown in Chapter 2 in Figure 2.4, where we change the sampling period of an inverted pendulum and measure the control cost.

As was investigated in Chapter 2, increasing the sampling period leads to better resource efficiency, since the controller executes less often and less resource bandwidth is required. However, increasing the sampling period also leads to worse control performance (higher control cost). This result is intuitive, since the more often the plant is controlled, the better the control performance, and the lower the control cost.

\subsection{Attack Model}

Observability and controllability are two concepts in control theory (Åström and Wittenmark, 1997) that are required for successful control of plants. The attack model we propose in this chapter is closely related to these two concepts. In fact, very little can be done to secure the system if it loses observability or controllability for a longer period of time (Cárdenas et al., 2008a). 
Intuitively, a system (including the attacker) is called observable if and only if the plant states can be observed both by the controller and by the intrusiondamage assessment and mitigation unit. Similarly, a system (including the attacker) is called controllable if and only if the plant states can be changed both by the controller and by the intrusion-damage assessment and mitigation unit.

Discarding or manipulating all sensor readings such that the actual state of the plant cannot be observed by the intrusion-damage assessment and mitigation unit is categorized as an unobservable attack. Similarly, discarding or manipulating all actuator commands such that the plant cannot be controlled by the controller and intrusion-damage assessment and mitigation unit is categorized as an uncontrollable attack.

In this chapter, we consider performance-observable and resource-controllable attacks. We define these notions more formally in the following.

Definition 3.3.1. Performance-observable attacks are those in which the performance degradation that is a result of the attack can be observed by the controller and the intrusion-damage assessment and mitigation unit.

Definition 3.3.2. Resource-controllable attacks are those in which allocating more resources to the task can compensate for the performance degradation that is a result of the attack.

Finally, we have two observations regarding the attacker's ability:

- The actual intrusion has a certain cost in terms of resources, e.g., time and power. Furthermore, the attacker is limited in terms of resources (time, energy, or computation power). Thus, there are, for example, certain limits in the capacity of the intruder to change the values of the packets per time unit.

- The attacker would like to remain undetected. Note that the intruder's goal is to have maximum impact on the physical plants, while remaining stealthy (Teixeira et al., 2015). This is often because non-stealthy attacks are immediately detected by the signature-based anomaly detection and the system enters an emergency mode (i.e., the attack will 


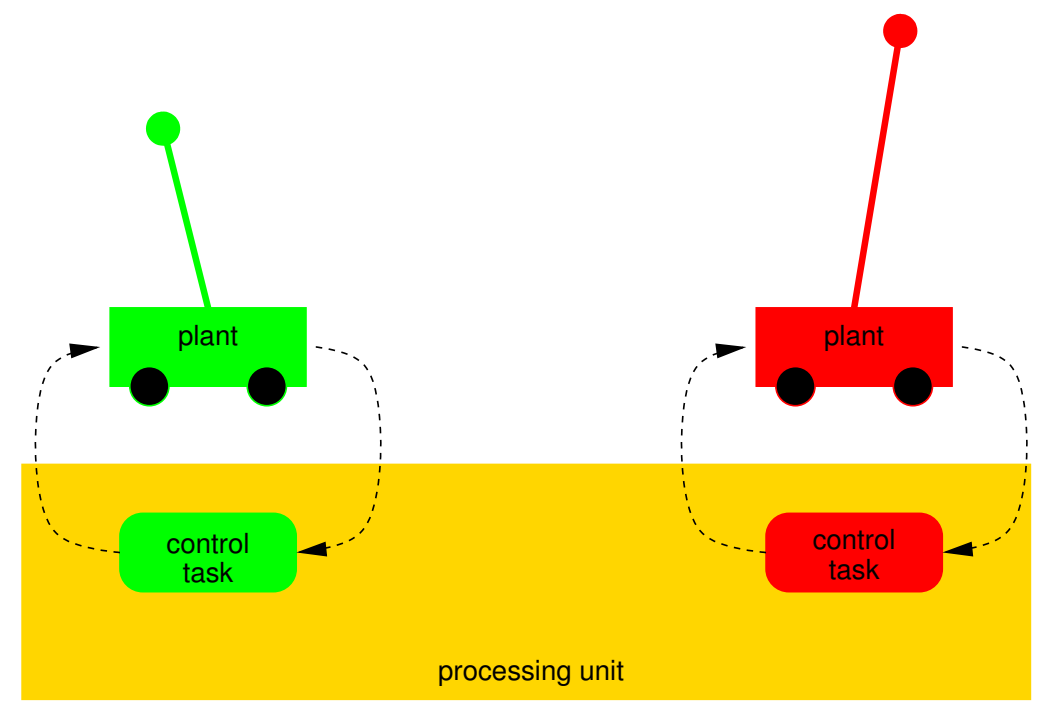

Figure 3.1: Two control applications sharing the same platform.

be unsuccessful). For example, if the intruder discards all sensor and/or actuator packets (or cuts the communication line), the signature-based anomaly detection mechanism can immediately start an emergency mode, in which, for instance, an emergency controller on the plant takes over, to facilitate the graceful and safe degradation of normal system operation.

\subsection{Motivational Example}

In this section, we shall demonstrate the problem using a small example. We shall elaborate on the same example in the rest of this chapter.

We consider a small system consisting of two inverted pendulums, as shown in Figure 3.1. Each pendulum is controlled by a single control task. The control tasks for both pendulums are implemented as software on a uniprocessor platform. Hence, the processing bandwidth of the uniprocessor is shared among the two control applications.

Each control task reads the state of the pendulum periodically with period $h$ and computes the control input that should be applied to the cart to keep the 


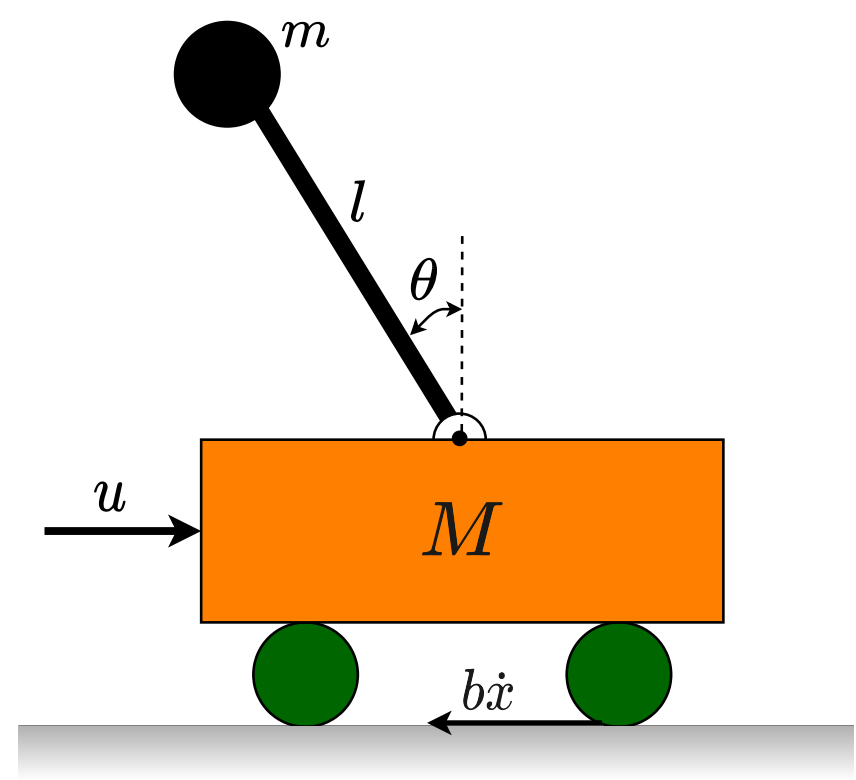

Figure 3.2: Schematic drawing of the inverted pendulum on a cart. $u$ is the control input and the force applied to the cart. $\theta$ is the pendulum angle from the upright position. The mass of the cart and the pendulum mass are denoted by $M$ and $m$, respectively. The rod has a length $l$ and $b$ is the friction coefficient of the cart.

pendulum and the cart as close as possible to the upright position and origin, respectively.

Let us consider the inverted pendulum system depicted in Figure 3.2. In order to design a controller for this pendulum one needs to use the dynamic equations of motion for the cart and pendulum system (Messner and Tilbury, 2017a). For the inverted pendulum plant $P_{i}$ the following equations are derived after applying the dynamic equations and summing all forces:

$$
\begin{gathered}
\left(M_{i}+m_{i}\right) \ddot{x}_{i}+b_{i} \dot{x}_{i}-m_{i} l_{i} \ddot{\theta}_{i} \cos \theta_{i}+m_{i} l_{i} \dot{\theta}_{i}^{2} \sin \theta_{i}=\boldsymbol{u}_{i}, \\
\left(I_{i}+m_{i} l_{i}^{2}\right) \ddot{\theta}_{i}-m_{i} g l_{i} \sin \theta_{i}=m_{i} l_{i} \ddot{x}_{i} \cos \theta,
\end{gathered}
$$

where the mass, the length, and the mass moment of inertia of the pendulum are denoted by $m_{i}, l_{i}$, and $I_{i}$, respectively. The mass and the friction coefficient of the cart are denoted by $M_{i}$ and $b_{i}$, respectively. $\boldsymbol{u}_{i}$ is the control 
Table 3.1: Specification of pendulum 1 and 2 .

\begin{tabular}{c|ccccc}
$P_{i}$ & $M_{i}$ & $m_{i}$ & $b_{i}$ & $l_{i}$ & $I_{i}$ \\
\hline$P_{1}$ & 0.5 & 0.2 & 0.1 & 0.3 & 0.006 \\
$P_{2}$ & 0.5 & 0.4 & 0.1 & 0.6 & 0.006
\end{tabular}

input and represents the force applied to the cart to keep the pendulum at upright position and keep the cart close to the origin. $\theta_{i}$ and $\dot{\theta}_{i}$ are the angle and angular velocity of the pendulum and $\ddot{\theta}_{i}$ is the second derivative of $\theta_{i}$. Analogously, $x_{i}$ and $\dot{x}_{i}$ are the position and velocity of the cart and $\ddot{x}_{i}$ is the second derivative of $x_{i}$. The plants' parameters for pendulums $P_{1}$ and $P_{2}$ are given in Table 3.1.

Assuming that the deviation of the pendulum from the upright equilibrium position is very small, we can use the following approximations to linearize the above equations,

$$
\begin{aligned}
\sin \theta_{i} & \approx \theta, \\
\cos \theta_{i} & \approx 1, \\
\dot{\theta}_{i}{ }^{2} & \approx 0 .
\end{aligned}
$$

Therefore, we can rewrite the Equation 3.3 as,

$$
\begin{gathered}
\left(M_{i}+m_{i}\right) \ddot{x}_{i}+b_{i} \dot{x}_{i}-m_{i} l_{i} \ddot{\theta}_{i}=\boldsymbol{u}_{i}, \\
\left(I_{i}+m_{i} l_{i}^{2}\right) \ddot{\theta}_{i}-m_{i} g l_{i} \theta_{i}=m_{i} l_{i} \ddot{x}_{i} .
\end{gathered}
$$

These linearized equations can be represented in state-space form as in Equation 2.1, with $\boldsymbol{A}_{i}$ and $\boldsymbol{B}_{i}$ matrices defined in the following,

$$
\boldsymbol{A}_{i}=\left[\begin{array}{cccc}
0 & 1 & 0 & 0 \\
0 & \frac{-\left(I_{i}+m_{i} l_{i}^{2}\right) b_{i}}{I_{i}\left(M_{i}+m_{i}\right)+M_{i} m_{i} l_{i}^{2}} & \frac{m_{i}^{2} l_{i}^{2}}{I_{i}\left(M_{i}+m_{i}\right)+M_{i} m_{i} l_{i}^{2}} & 0 \\
0 & 0 & 0 \\
0 & \frac{-m_{i} b_{i}}{I_{i}\left(M_{i}+m_{i}\right)+M_{i} m_{i} l_{i}^{2}} & \frac{m_{i} g l_{i}\left(M_{i}+m_{i}\right)}{I_{i}\left(M_{i}+m_{i}\right)+M_{i} m_{i} l_{i}^{2}} & 0
\end{array}\right], \boldsymbol{B}_{i}=\left[\begin{array}{c}
0 \\
\frac{I_{i}+m_{i} l_{i}^{2}}{I_{i}\left(M_{i}+m_{i}\right)+M_{i} m_{i} l_{i}^{2}} \\
0 \\
\frac{m_{i} l_{i}}{I_{i}\left(M_{i}+m_{i}\right)+M_{i} m_{i} l_{i}^{2}}
\end{array}\right] .
$$

The plant state vector for the inverted pendulum $P_{i}$ is $\left[x_{i}, \dot{x}_{i}, \theta_{i}, \dot{\theta}_{i}\right]^{T}$. The $\boldsymbol{D}_{i}$ matrix is considered to be zero and the $\boldsymbol{C}_{i}$ matrix is assumed to be the identity matrix, therefore the output of the pendulum is equal to the pendulum state plus the measurement noise.

The goal of the controller is to minimize the deviation of the pendulum from the upright position and the deviation of the cart from the origin, also taking 
3. Intrusion Detection and Mitigation for Control

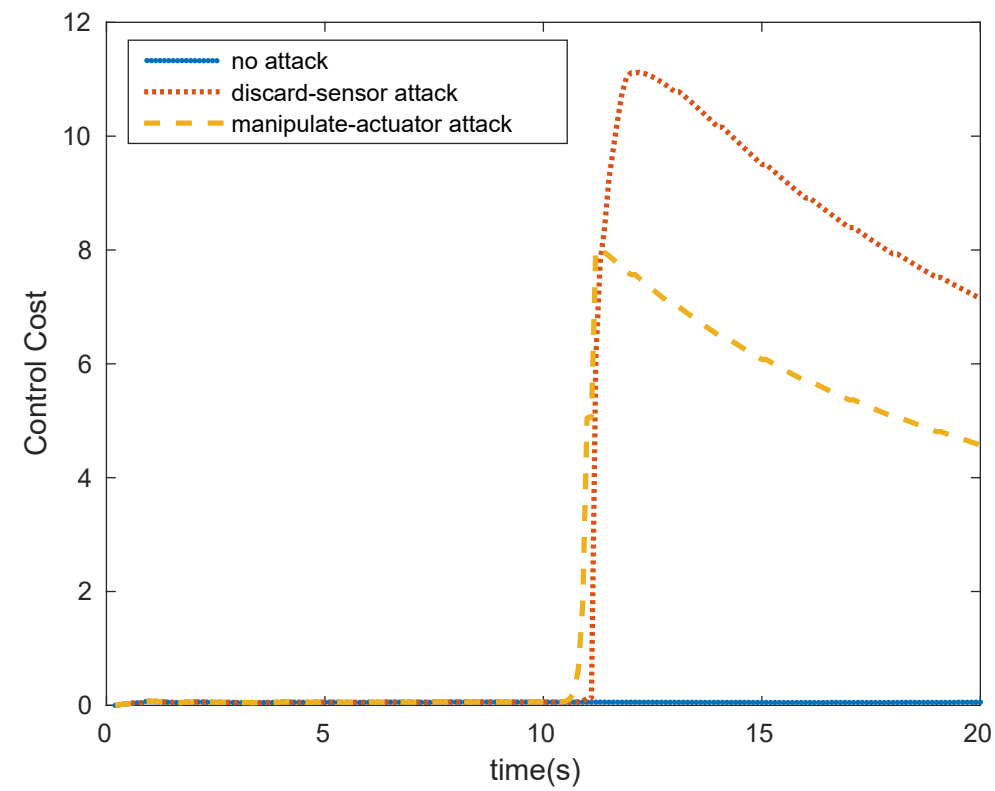

Figure 3.3: Control cost of pendulum under different attacks.

into account the energy required. We design an optimal state-feedback controller, Linear-Quadratic-Gaussian (LQG), assuming the linearized equations introduced above, as follows,

$$
\boldsymbol{u}_{i}=\boldsymbol{K}_{i}\left[\begin{array}{c}
x_{i} \\
\dot{x_{i}} \\
\theta_{i} \\
\dot{\theta}_{i}
\end{array}\right],
$$

where $\boldsymbol{K}_{i}$ is the LQG controller for pendulum system $P_{i}$. Note that the initial sampling frequency for pendulum 1 and 2 are estimated from Equation 3.2 and are $f_{1}=9$ and $f_{2}=11$.

In the following we consider two attack scenarios on the green pendulum $P_{1}$. The control performance degradation for each scenario is calculated according to Equation 2.3 and is shown in Figure 3.3. The scenario in which there is no attack is shown by the blue line, in which the control cost is close to zero. 
The first attack scenario is based on discarding sensor readings. The attacker discards 9 successive sensor readings, starting at time 10. In the second scenario, the attacker multiplies 9 successive actuator commands, starting at time 10 , by a factor of 1.8. Note that, for both scenarios, the performance degradation (increased control cost) is observed immediately after the attack at time 10.6. Comparing the two attacks, we realize that discarding 9 sensor readings deteriorates the control performance of the inverted pendulum more than multiplying 9 actuator commands by a factor of 1.8 , for the given state at time 10 .

We conclude this section by making two observations. First, the attacker can degrade the quality of control and, in the worst case, can even jeopardize the stability of the application. Secondly, the degradation in the control application's performance can be utilized to assess the damage caused by the attack. We shall elaborate on the latter in Section 3.6.1.

\subsection{Problem Formulation}

In this section, we shall formulate the problem to be addressed in this chapter. The proposed approach to address this problem will be illustrated in the following section.

We consider a uniprocessor platform, shared among several applications as described in Section 2.1.1, where a subset of these applications are periodic control applications. We assume the following inputs for each control application $\Lambda_{i}$,

- a model of the plant $P_{i}$ and the controller task $\tau_{i}$;

- the worst-case execution time $c_{i}$ and period $h_{i}$ of the controller task; and

- the sampling period interval within which the control application can provide satisfactory performance.

We consider a scenario in which one or more of the control applications might be under attack. We focus on performance-observable and resourcecontrollable attacks, introduced in Section 3.3. 


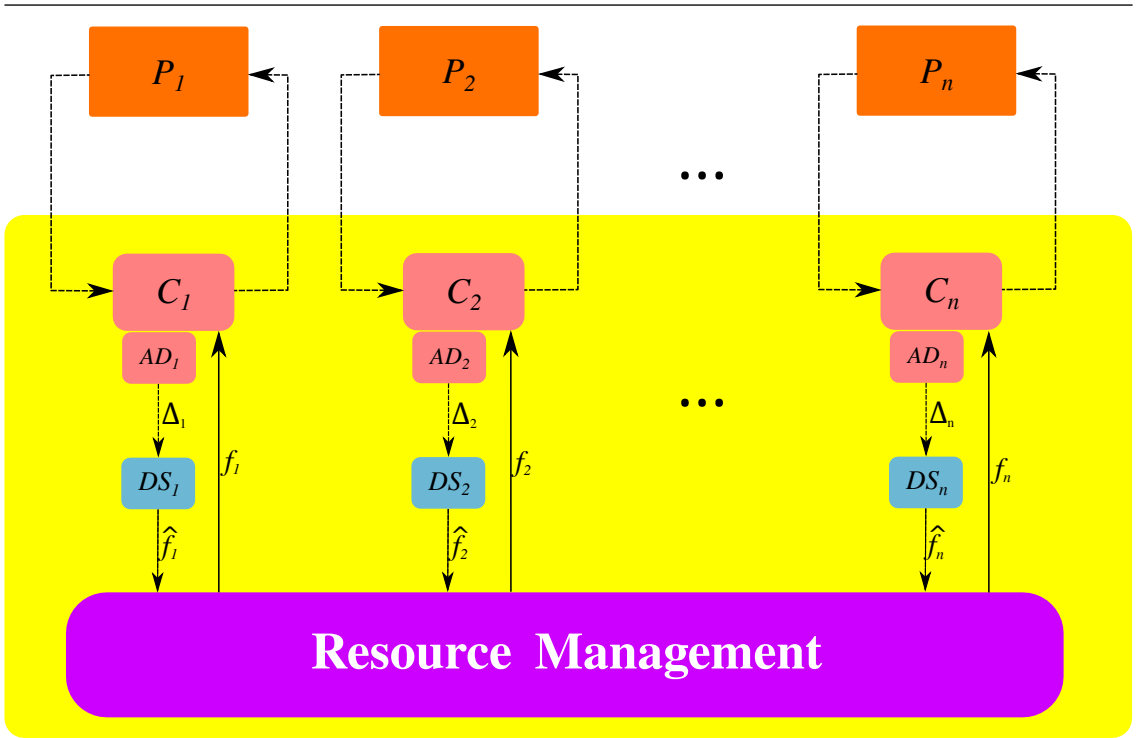

Figure 3.4: Overview of our approach.

Our goal is to assess the intrusion-generated damage and mitigate the impact of the attacks through careful resource management.

\subsection{Approach}

In this section, we illustrate our intrusion-damage assessment and mitigation techniques.

The overall view of our proposed approach is shown in Figure 3.4. As mentioned, we have a uniprocessor platform shared among several control applications. Each plant $P_{i}$ is controlled by a controller $C_{i}$, implemented as a software control task $\tau_{i}$. There is also an intrusion-damage assessment unit associated with each control application, denoted by $A D_{i}$ ( $A D$ stands for anomaly detection). The intrusion-damage assessment unit uses the sensor and actuator values received and sent by the control task to cross-check the observed behavior against what is expected based on the plant model. In Section 3.6.1, we further discuss our intrusion-damage assessment technique, which is based on a machine learning technique. 
The output $\Delta_{i}$ of the intrusion-damage assessment unit captures the damage generated by the intrusion and is sent to the desired sampling unit $D S_{i}$. This unit then decides what the desired sampling frequency $\hat{f}_{i}$ for this control application should be in order to mitigate the damage generated by the intrusion. The desired sampling frequency $\hat{f}_{i}$ is sent to the resource management unit, which gathers the desired sampling frequencies for all control applications. The resource manager then allocates the right amount of resources to each application and runs the tasks with the new sampling frequencies $f_{i}$. The desired sampling units, together with the resource manager, constitute the intrusion-damage mitigation unit. In Section 3.6.2, we discuss our intrusion-damage mitigation technique, formulated as an optimization problem, which is designed to mitigate the generated intrusion-damage by careful management of the available resources.

\subsubsection{Intrusion-Damage Assessment}

As discussed in Section 3.3, with the exception of the observability and controllability, we do not have any particular assumptions about the attack scenarios. This basically means that our approach cannot rely on the use of data regarding patterns of attack scenarios at design time.

To detect intrusion, we have to identify the boundary between the normal samples/observations and the abnormal samples/observations that are due to the attack. Now, generally speaking, there are three ways to identify the boundary between the normal and abnormal observations.

The first approach is to have a description of the abnormal behavior. However, to have a description of the abnormal behavior, it is basically necessary to have data about various attack scenarios. This is effectively what is done in signature-based anomaly detection. The second approach to identifying the boundary is to have data samples from both normal behavior and abnormal behavior of the system. This is basically what is done with classification techniques. Both of these approaches assume availability of data samples of the abnormal behavior and are not effective in the presence of new, unanticipated, or zero-day attack scenarios. 
The previously discussed approaches are not applicable in our case since we cannot gather data from the attack scenarios, as this would require us to restrict ourselves to certain specific attacks. Therefore, we have employed a third approach.

Our approach is to consider the normal behavior of the system and identify anomalies with respect to this normal behavior. A major challenge for anomaly detection is capturing the normal behavior. In our case, however, the existing mathematical model of the plant, in Equation 2.1, can capture the normal behavior of the system, to a great extent. Of course, by definition, no model is exact, so we use a machine learning algorithm to capture the deviation of the expectation based on the mathematical model from the actual observed value in normal situations.

In our anomaly detection approach, the boundary between the normal behavior and the anomalous behavior is not known (Chandola et al., 2009), due to the fact that we cannot assume that the plant model is $100 \%$ accurate. Therefore, it is reasonable to use the intrusion-damage assessment terminology (which suggests a real value output, capturing the discrepancy between the observed value and the expected value according to the model) rather than intrusion detection (which suggests a binary value output, distinguishing safe and unsafe situations). Accordingly, the greater the deviation between the observed and expected values, the higher the intrusion-damage, and the higher the probability of intrusion.

The intrusion-damage assessment has two main phases: (1) offline learning phase, and (2) online evaluation phase.

\subsubsection{Offline Learning}

To capture the deviation between the expected and observed behaviors, we use the probability ellipsoid approach described in (Ribeiro, 2004). The set of features used are $k$ previous outputs of the plant, i.e. $\tilde{\boldsymbol{y}}(i-k), \ldots, \tilde{\boldsymbol{y}}(i-1)$, and the difference between the current output of the plant calculated based on the mathematical model $\boldsymbol{y}(i)$ and the current observed plant output $\tilde{\boldsymbol{y}}(i)$, 


$$
\boldsymbol{X}=\left[\begin{array}{c}
\tilde{\boldsymbol{y}}(i-k) \\
\cdots \\
\tilde{\boldsymbol{y}}(i-1) \\
\boldsymbol{y}(i)-\tilde{\boldsymbol{y}}(i)
\end{array}\right]
$$

Note that $k$ is a parameter that captures the importance of historical plant output and can be adjusted based on the application. By increasing $k$ one can take history over a longer interval into consideration for better intrusion assessment.

In the learning phase, we run several experiments to obtain a set of feature vectors $\boldsymbol{X}$ as in Equation 3.8, i.e., the $k$ previous outputs of the plant, $\tilde{\boldsymbol{y}}(i-k)$, $\ldots, \tilde{\boldsymbol{y}}(i-1)$, and the difference between the current plant output, calculated based on the mathematical model $\boldsymbol{y}(i)$, and the current observed plant output $\tilde{\boldsymbol{y}}(i)$.

Having obtained the large set of feature vectors, we can now estimate the values of mean vector $\boldsymbol{\mu}$ and covariance matrix $\boldsymbol{\Sigma}$ as follows,

$$
\boldsymbol{\mu}=\frac{1}{N} \sum_{j=1}^{N} \boldsymbol{X}^{(j)}, \quad \boldsymbol{\Sigma}=\frac{1}{N-1} \sum_{j=1}^{N}\left(\boldsymbol{X}^{(j)}-\boldsymbol{\mu}\right)\left(\boldsymbol{X}^{(j)}-\boldsymbol{\mu}\right)^{T},
$$

where $N$ is the total number of samples and the superscript indicates the sample number.

\subsubsection{Online Evaluation}

Given the previous output of the plant $\tilde{\boldsymbol{y}}(i-1)$, based on the mathematical model in Equation 2.1 and the time elapsed since the last activation of the intrusion-damage assessment and mitigation unit, we compute the current output of the plant $\boldsymbol{y}(i)$. Using this in combination with the observed plant outputs $\tilde{\boldsymbol{y}}(i-k), \ldots, \tilde{\boldsymbol{y}}(i)$, we can now compute the feature vector $\boldsymbol{X}$ according to Equation 3.8.

It can be shown that, since the noise and disturbance in Equation 2.1 follow independent normal distributions (Åström and Wittenmark, 1997), the output of the system described in Equation 2.1 also follows a normal distribution (Lindgren, 2012). In addition, the difference of two random variables with normal distributions also follows a normal distribution (Weisstein, 2016). 
That said, and assuming that $\boldsymbol{\mu}$ and $\boldsymbol{\Sigma}$ are the mean vector and covariance matrix for feature vector $\boldsymbol{X}, \boldsymbol{X} \sim N(\boldsymbol{\mu}, \boldsymbol{\Sigma})$, then the scalar random variable $Q$ defined by the quadratic form

$$
Q=(\boldsymbol{X}-\boldsymbol{\mu})^{T} \boldsymbol{\Sigma}^{-1}(\boldsymbol{X}-\boldsymbol{\mu}),
$$

follows $\chi_{k}^{2}$ distribution with $k$ degrees of freedom, where $k$ is the size of feature vector $\boldsymbol{X}$ (Ribeiro, 2004). Then, the cumulative distribution function of $\chi_{k}^{2}$ is as follows (Montgomery et al., 2009),

$$
\Delta=\frac{\gamma\left(\frac{k}{2}, \frac{U}{2}\right)}{\Gamma\left(\frac{k}{2}\right)}
$$

where $\Gamma($.$) and \gamma($.$) are defined below$

$$
\Gamma(s)=\int_{0}^{\infty} t^{s-1} e^{-t} d t, \quad \gamma(s, x)=\int_{0}^{x} t^{s-1} e^{-t} d t .
$$

Equation 3.11 captures the inconsistency between the observations and the expectation, on a scale of 0 to 1 .

The greater the intrusion-generated damage is, the greater the deviation of the observed behavior from the expected behavior, and the higher the value of $\Delta$ in Equation 3.11. Therefore, in the following, we shall refer to the output of the intrusion-damage assessment unit, given by Equation 3.11, as intrusiongenerated damage.

The time complexity of the proposed intrusion-damage assessment mechanism corresponds to:

- the evaluation of the current state of the plant based on the mathematical model from the previous state of the plant, and

- the evaluation of Equation 3.8, Equation 3.10, and Equation 3.11.

Both of the above steps can be done in polynomial time. Hence the efficiency of our proposed intrusion-damage assessment mechanism.

\subsubsection{Intrusion-Damage Mitigation}

As discussed previously, we use a careful resource allocation mechanism to perform intrusion-damage mitigation. The fundamental idea is illustrated in 
Section 2.2 and Figure 2.4, that is, allocating more resources to a control application, in general, leads to a higher quality of control and performance.

This approach is of particular interest because of its applicability to many scenarios and its flexibility and adaptivity. Hence, using a careful resource allocation mechanism, it is possible to mitigate the impacts of several kinds of attacks, even if these attacks are not known to the designer during the system design phase.

The intrusion-damage mitigation unit has two main components (see Figure 3.4) (1) desired sampling unit, and (2) resource management unit.

\subsubsection{Desired Sampling Unit}

For each control application we have a desired sampling unit, indicated by $D S$ in Figure 3.4. This unit receives, as an input, the intrusion-generated damage $\Delta$ from the intrusion-damage assessment unit. The output is the desired sampling frequency $\hat{f}$ that would allow the control application to mitigate the intrusion-damage, ignoring the resource constraints and other applications in the system. That is, this unit assumes the ideal case of a dedicated resource and computes the desired sampling frequency, ignoring all other applications. In cases where intrusion-damage $\Delta$ is close to zero, the desired sampling unit outputs the initial sampling frequency $f^{(0)}$ defined by the designer of the system.

The idea is similar to that of feedback control. Iteratively, this unit computes the desired sampling frequency $\hat{f}^{(j)}$ based on the previous frequency $f^{(j-1)}$, current intrusion-generated damage $\Delta^{(j)}$, and the difference between the current intrusion-generated damage and the previous one $\Delta^{(j)}-\Delta^{(j-1)}$ as follows,

$$
\hat{f}^{(j)}=\left(1-S\left(\Delta^{(j)}\right)\right) f^{(0)}+S\left(\Delta^{(j)}\right)\left(f^{(j-1)}+\alpha \Delta^{(j)}+\beta\left(\Delta^{(j)}-\Delta^{(j-1)}\right)\right),
$$

where $S($.$) is a sigmoid function between 0$ and 1 . Note that, $\alpha, \beta$, and $S($. can be tuned based on the specific control application. The desired sampling frequency is close to $f^{(0)}$ when $\Delta^{(j)}$ is close to zero. In the case that $\Delta^{(j)}$ is close to 1 , the desired frequency is computed based on feedback, which means 
frequency increases/decreases linearly as the intrusion-generated damage and the rate of intrusion-generated damage increase/decrease.

\subsubsection{Resource Management Unit}

Given the desired sampling frequencies $\hat{f}_{i}$ for all control applications, the task of the resource management unit is to allocate the available processing power among these applications, such that the deviation between the desired resources and allocated resources is minimized.

This is formulated in the following optimization problem,

$$
\begin{array}{cl}
\min _{f_{1}, \ldots, f_{n}} & \sum_{i=1}^{n} \omega_{i} \cdot\left(f_{i}-\hat{f}_{i}\right)^{2} \\
\text { s.t. } & \sum_{i=1}^{n} c_{i} \cdot f_{i} \leqslant U, \\
& \underline{f}_{i} \leqslant f_{i} \leqslant \bar{f}_{i}, \quad \forall i=1 \ldots n,
\end{array}
$$

where coefficients $\omega_{i}$ capture the relative importance of the control applications and $c_{i}$ are the worst-case execution times for the control tasks. The objective of the optimization problem is, as previously discussed, to minimize the cumulative distance between the desired sampling frequencies and the assigned sampling frequencies. If we set $U=1$, the first constraint ensures real-time schedulability of the control applications, assuming an earliest-deadline-first (EDF) scheduling policy (Liu and Layland, 1973). The minimum frequency required to guarantee a certain quality of control for control task $\tau_{i}$ is denoted by $\underline{f}_{i}$, and is calculated based on Equation 3.2. The maximum frequency is denoted by $\bar{f}_{i}$, and for each task $\tau_{i}$ is determined by setting the minimum frequency for other tasks and using the schedulability condition in Equation 3.13.

Observe that the above optimization problem is convex and can be solved efficiently in polynomial time, hence the applicability of our approach in a resource-constrained setting.

\subsubsection{Experimental Example}

In this subsection, we use the motivational example illustrated in Section 3.4 to experimentally evaluate the intrusion-damage assessment and mitigation approaches. 


\subsubsection{Experimental Setup}

To run the experiment we simulate the shared platform with a simulation software in Matlab. We simulate each pendulum based on the non-linear (more accurate) model described in Equation 3.3. We also simulate the input and output noise of the pendulum by a Gaussian white noise. Furthermore, we use the linear model of the pendulum described in Equation 3.5 as the mathematical model to design the controller and calculate proper control input. Basically, for pendulum $P_{i}$ we receive the observed plant output $\tilde{\boldsymbol{y}}_{i}$ from the simulation and use the LQG controller, based on the approximate linear model, to calculate proper control input $\boldsymbol{u}_{i}$.

In the offline learning phase for each and different sampling periods we run the simulation for 1000 iterations and obtain a large set of feature vectors. Then we use the feature vectors to calculate mean vector $\boldsymbol{\mu}$ and covariance matrix $\Sigma$ as described in Equation 3.9.

Then, during the online evaluation phase, for pendulum $P_{i}$, in each iteration of the simulation the controller extracts the feature vector and then the $A D_{i}$ unit uses this feature vector to calculate $\Delta_{i}$ as described in Equation 3.11. In the intrusion-damage mitigation phase, the desired sampling unit $\left(D S_{i}\right)$, calculates the desired frequency for plant $P_{i}$ and finally the resource management unit calculates the proper frequency as illustrated in Equation 3.13.

\subsubsection{Evaluation of Intrusion-Damage Assessment}

Here we evaluate our intrusion-damage assessment approach. We focus on the green pendulum $P_{1}$ in our example. There are two attack scenarios in this example, discarding actuator commands and manipulating sensor readings. Note that, in this section, we only assess the damage generated by the attack. The next section focuses on mitigating the damage generated by the attacker.

In the first attack scenario the attacker discards between 4 and 10 actuator commands, starting at time 10 (start of attack is shown by a vertical black line in Figure 3.5 and Figure 3.6. The intrusion-generated damage produced by our intrusion-damage assessment unit is shown in Figure 3.5a. Observe that the more powerful the attack is, the larger the intrusion-generated damage would be. Also, the assessment unit reacts to the attack and the intrusion-generated 


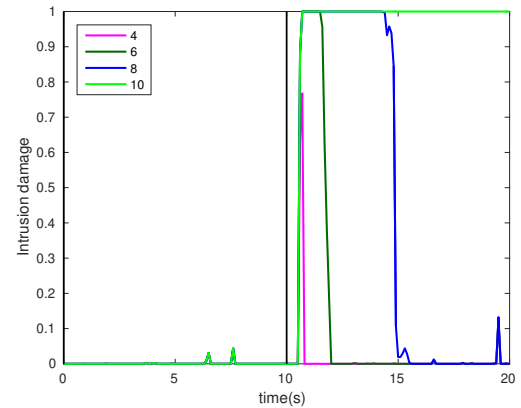

(a) Discard 4-10 actuator commands.

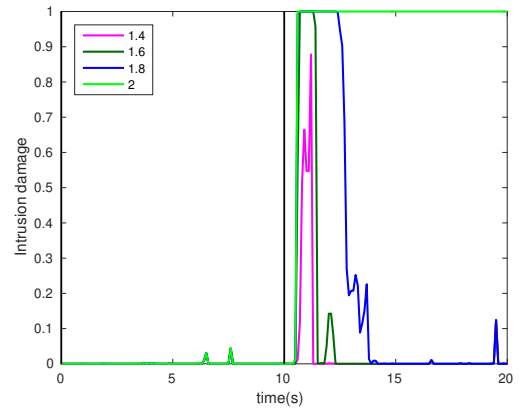

(b) Manipulate sensor readings.

Figure 3.5: Intrusion-generated damage for the pendulum $P_{1}$ during two attack scenarios; no intrusion mitigation in operation.

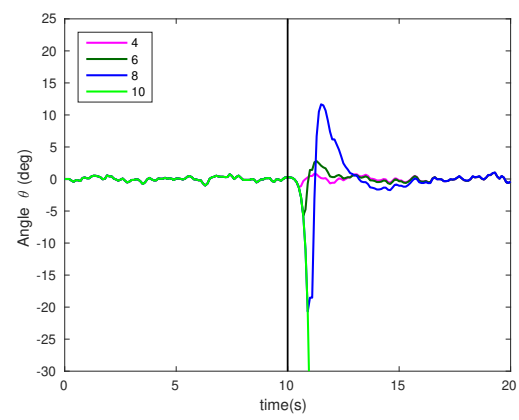

(a) Discard 4-10 actuator commands.

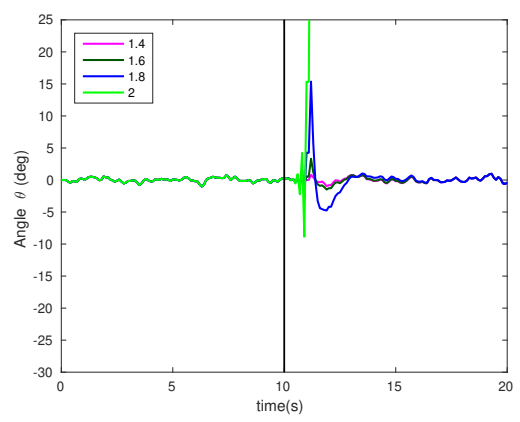

(b) Manipulate sensor readings.

Figure 3.6: Deviation of the pendulum $P_{1}$ from upright position (angle $\theta$ ) during two attack scenarios; no intrusion mitigation in operation. 


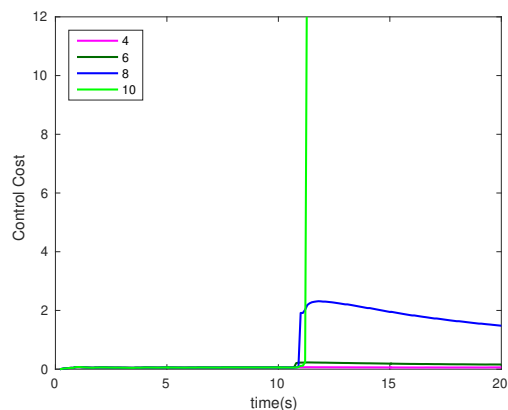

(a) Discard 4-10 actuator commands.

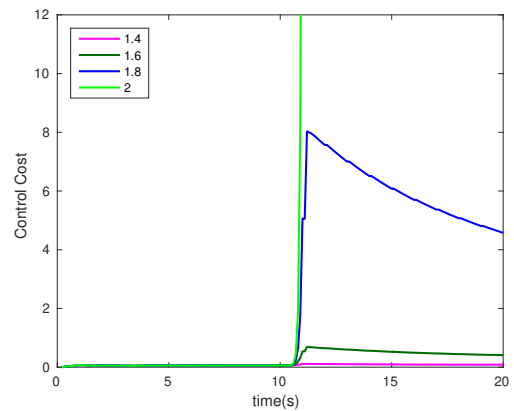

(b) Manipulate sensor readings.

Figure 3.7: Control cost for the pendulum $P_{1}$ during two attack scenarios; no intrusion mitigation in operation.

damage reaches 0.6 immediately after the attack at time 10.6. Figure 3.6a shows the deviation of the pendulum from the upright position as a result of this attack. We observe that the intrusion-generated damage (see Figure 3.5a) is consistent with the deviation of the pendulum from the upright position (see Figure 3.6a). Figure 3.7a shows the performance degradation of the control application as a result of these attacks. Note that in Figure 3.5, Figure 3.6, and Figure 3.7 the lines for the different attacks are overlapping before we start to see the effect of attack, hence only the green line is visible in the figures.

The second attacker manipulates 9 sensor readings by a factor between 1.4 and 2.0, starting at time 10. The intrusion-generated damage, the deviation of the pendulum from the upright position, and the performance degradation for the control application are shown in Figure 3.5b, Figure 3.6b, and Figure 3.7b, respectively.

Observe that discarding 10 actuator commands in the first attack scenario or manipulating 9 sensor readings by a factor of 2.0 in the second scenario causes the pendulum to fall over. It is shown in the green diagram in Figure 3.6 that the pendulum deviates to angles larger than 180 degrees for both attack types. In Figure 3.7, the control cost of the green diagram tends toward infinity and the intrusion-generated damage in Figure 3.5 remains at 1. 
To conclude this subsection, we make three observations. Firstly, we are able to efficiently assess the damage generated by the intruders. Secondly, the assessment mechanism is not restricted to a specific attack scenario. For example, we discussed both discarding actuator commands and manipulating sensor readings. Thirdly, the more powerful the attack is the larger our intrusion-damage metric gets and the more the pendulum deviates from the origin. Indeed, comparing Figure 3.5 and Figure 3.6, the intrusion-damage assessment unit assesses high intrusion-damage whenever the pendulum angle is large.

\subsubsection{Evaluation of Intrusion-Damage Mitigation}

Here we evaluate our intrusion-damage mitigation approach. We consider two attack scenarios as before. The first attack is discarding actuator commands and the second one is manipulating sensor readings, with both attacks on pendulum $P_{1}$ starting at time 10 . Note that for both attacks, we are considering the most powerful attack in Section 3.6.1, i.e., discarding 10 actuator commands for the first attack and manipulating 9 sensor readings by a factor of 2.0 for the second one. The main difference in this section is that our intrusion-damage mitigation unit is active. The coefficients $\omega_{i}$ and execution times $c_{i}$ are considered the same for both pendulums, $\omega_{1}=\omega_{2}=1$ and $c_{1}=c_{2}=0.05$. Also, the minimum and maximum frequencies for the both pendulums are $\underline{f}_{1}=\underline{f}_{2}=5.6$ and $\bar{f}_{1}=\bar{f}_{2}=14.4$.

Figure 3.8 shows the intrusion-damage when the mitigation unit is activated. It can be observed that the intrusion-damage is also propagated to the second pendulum. This pendulum is not under attack, but due to the fact that the sampling frequency of the pendulum $P_{1}$, which is under attack, is increased (see Figure 3.11), the sampling frequency of pendulum $P_{2}$ is decreased. However, as shown in Figure 3.10a, the control performance degradation for the second pendulum is negligible. The important aspect, however, is that the overall damage caused by the intrusion is kept under control.

In Figure 3.9b, we observe that the pendulum will not be affected at all by the second attack, compared to Figure $3.6 \mathrm{~b}$, in which the pendulum falls over. For the first attack in Figure 3.9a, the deviation of the pendulum from the 


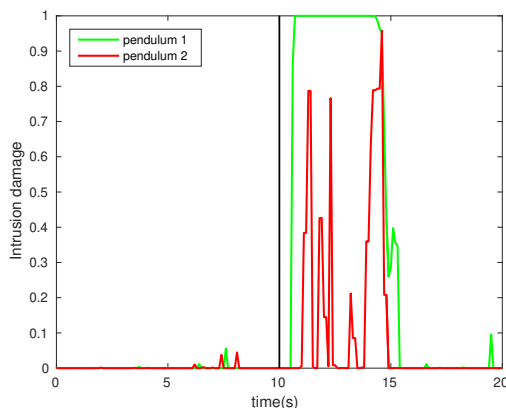

(a) Discard 10 actuator commands.

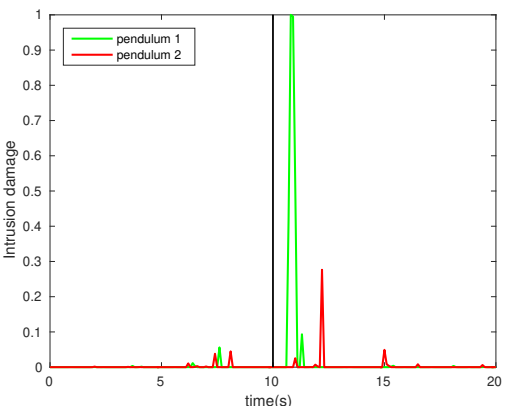

(b) Manipulate sensor readings.

Figure 3.8: Intrusion-generated damage calculated for the two pendulums during two attack scenarios while intrusion mitigation is functioning.

upright position is less than 25 degrees, compared to Figure 3.6a, in which the pendulum falls over.

Figure 3.10 demonstrates the efficiency of our proposed approach in terms of overall control cost. The control cost increase as a result of the first attack is less than 3 in Figure 3.10a, compared to Figure 3.7a, which shows control cost approaching infinity for an attack with the same power. In particular, comparing the light green lines in Figure 3.7b and Figure 3.10b shows that the impact of the second attack is mitigated to a great extent, as the control cost for this attack is reduced from infinity (since the pendulum falls over) to almost zero. Observe that since we allocate more resources to the pendulum under attack, the second pendulum might experience some performance degradation (increase in the control cost).

Finally, Figure 3.11 depicts how resources are allocated to the control applications over time. Note that in Figure 3.11a, when the attack occurs on the green pendulum, the intrusion-damage mitigation unit allocates more resources (higher sampling frequency) to this pendulum and reduces the resources allocated to the second pendulum by decreasing its sampling frequency. A similar scenario occurs in Figure 3.11b, but for a shorter period of time. Observe that the considerably smaller frequency change around time 


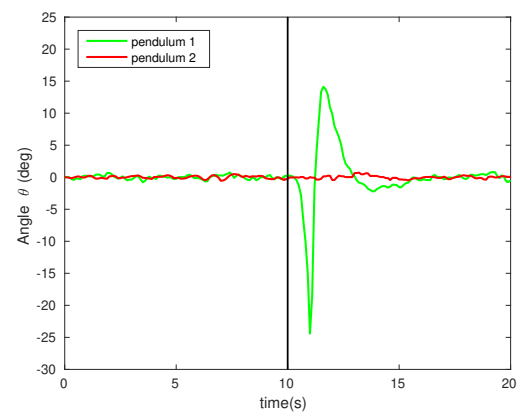

(a) Discard 10 actuator commands.

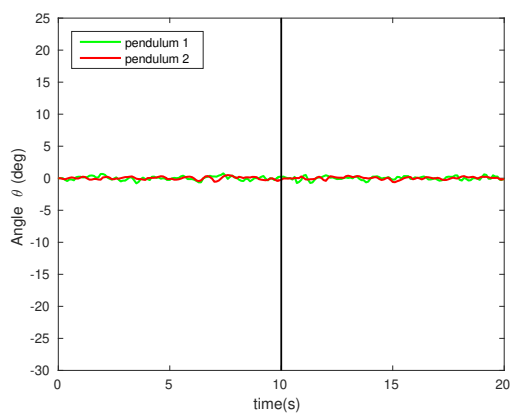

(b) Manipulate sensor readings.

Figure 3.9: Deviation of the two pendulums from upright position (angle $\theta$ ) during two attack scenarios while the intrusion mitigation is in operation.

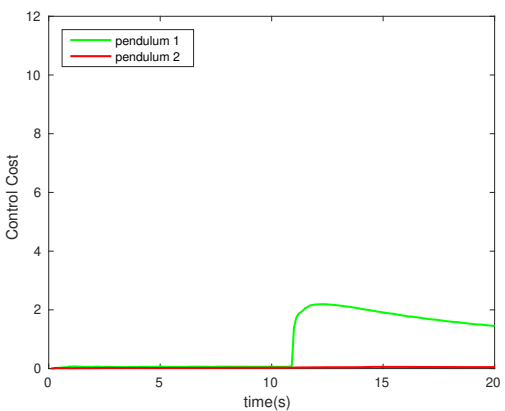

(a) Discard 10 actuator commands.

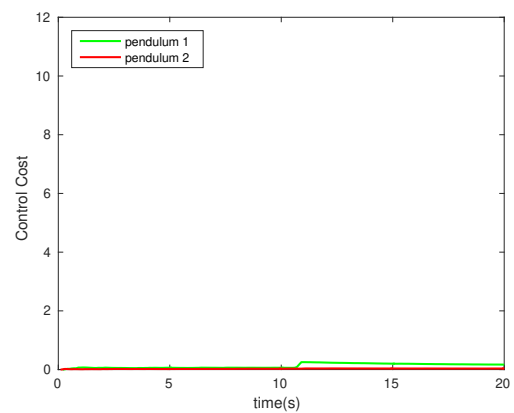

(b) Manipulate sensor readings.

Figure 3.10: Control cost for the two pendulums during two attack scenarios while the intrusion mitigation is in operation. 


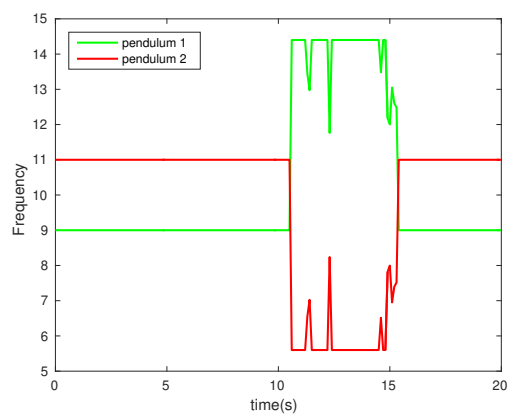

(a) Discard 10 actuator commands.

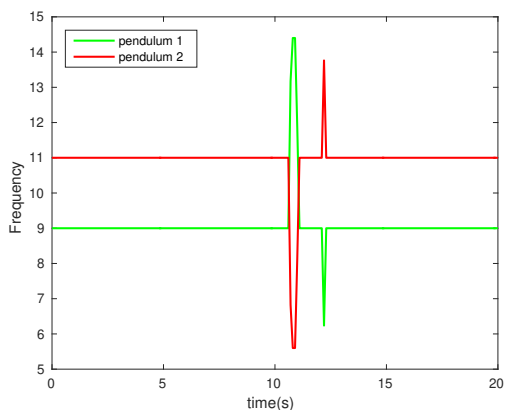

(b) Manipulate sensor readings.

Figure 3.11: Allocation of resources (in terms of sampling frequency) for the two pendulums during two attack scenarios.

12 in Figure 3.11a is because of an improbable amount of noise in actuator commands and sensor readings.

Note that although we have demonstrated our approach using a small example, we do not have any restricting assumption on the number of applications, the different kinds of plants, or attack scenarios (as long as they are observable and controllable). Additionally, even though we consider attacks only on the first pendulum, the second pendulum might also be attacked, with no restriction. Of course, as discussed in Section 3.3, if our system does not have enough resources to mitigate the intrusion-generated damage, we activate an emergency mode, in which, for instance, an emergency controller on the plant takes over, to facilitate the graceful and safe degradation of normal system operation.

\subsection{Conclusions}

In this chapter, we highlighted several important properties of control applications in CPSs. We defined the notion of observable and controllable attacks. Based on the highlighted properties of control applications, we proposed a systematic intrusion-damage assessment and mitigation mechanism for this class of attacks. The experiments in this chapter confirmed the validity of our intrusion-damage assessment and mitigation approach. 


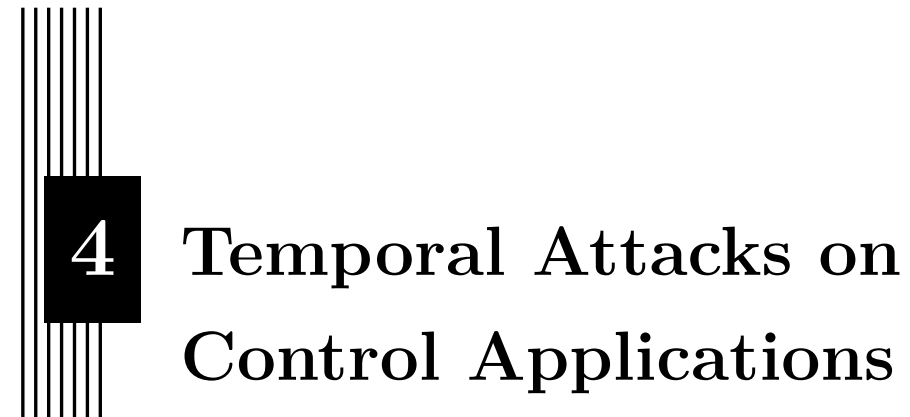

Cyber-physical systems are tightly integrated with networks and components (Lee, 2008), giving an adversary ample opportunities to provide malicious input, potentially compromising the safety of the system. Unfortunately, many such systems comprise critical components, e.g., adaptive cruise control or engine control in automotive systems, that allow deep intervention in the driving of a vehicle (Lemke et al., 2006). Therefore, ignoring security in the design of CPSs can have severe consequences. Additionally, as discussed in Chapter 1, these systems often comprise multiple control applications that are implemented on shared platforms. Such resource sharing may lead to complex timing behaviors and, in turn, counter-intuitive timing anomalies that can be exploited by adversaries to destabilize a critical control system, resulting in irreversible consequences. In this chapter, RQ2 (see Section 1.2) is investigated. We introduce the Butterfly attack, a new attack scenario against CPSs that carefully exploits the sensitivity of control applications with respect to the implementation on the underlying execution platforms. We illustrate the potential for such attacks using two case studies from the automotive and avionic domains. The content of this chapter is based on the following publication: (Mahfouzi et al., 2019b). 


\subsection{Introduction and Related Work}

As indicated in Chapter 1, CPSs are shifting towards integrated architectures, as observed, e.g., in the automotive domain (Di Natale and Vincentelli, 2010). As a result, the majority of control applications in such domains are implemented as software tasks on commodity platforms, sharing the CPU with other tasks. If not carefully taken into account, this resource sharing leads to complex timing behaviors, which may jeopardize the stability of the control applications (Årzén and Cervin, 2005; Cervin et al., 2019; Wittenmark et al., 1995).

Temporal attacks are a new class of attacks in which the correct behavior of the system is targeted through manipulation of its temporal properties. For example, execution overruns of an application could lead to deadline misses in other applications. Such deadline misses, in turn, could have catastrophic consequences in safety-critical applications. Following best practices, overruns are considered in the design phase and appropriate measures are planned for such cases. However, as we discuss later in this chapter, temporal attacks are by no means limited to overruns. In fact, there are non-trivial cases in which the safety of a system is jeopardized if a non-critical task simply stops executing. In control applications, this could lead to longer jitter in execution of the critical control task and instability of the physical plant. Note that although the non-critical application does not violate its limit on resource usage, the physical plant associated with the critical control application is compromised. Similarly and counter-intuitively, if a task decides to switch to a lower priority level or a longer period, i.e., giving up certain privileges, the stability of other control applications can be compromised.

The timing problems in real-time control systems were first brought up by Wittenmark et al. (Wittenmark et al., 1995). The authors discuss the issue of time-varying delays introduced during the implementation phase of such systems, which often lead to poor control performance and may even jeopardize the stability of control applications. Since then, several studies have considered implementation-induced timing issues in the design of such embedded control and CPSs (Aminifar et al., 2015a,b, 2013a, 2018; Årzén et al., 2000; Behnam and Isovic, 2007; Bini and Cervin, 2008; Biondi and Buttazzo, 2018; Biondi et al., 2017; Bund and Slomka, 2013; Cervin et al., 2004; Chang et al., 
2018; Dhruva et al., 2014; Fontanelli et al., 2013a; Fontanelli et al., 2013b; Fontantelli et al., 2013; Gaid et al., 2006; Goswami et al., 2012; Kumar et al., 2012; Lemmon et al., 2007; Mancuso et al., 2011; Mancuso et al., 2014; Mohaqeqi et al., 2018; Naghshtabrizi and Hespanha, 2009; Nghiem et al., 2006; Palopoli et al., 2005; Rehbinder and Sanfridson, 2000; Samii et al., 2009; Seto et al., 1996; Xu et al., 2015, 2014; Zhang et al., 2008; Ziegenbein and Hamann, 2015).

Authors in (Racu and Ernst, 2006) show that, contrary to what is widely believed, a shorter execution time for one task in a task chain, mapped on a multicore platform, may lead to a longer end-to-end delay for the same task chain, in the context of purely hard real-time systems. Aminifar et al. (Aminifar et al., 2013b) demonstrate that intuitively better design parameters, e.g., priority, period, and execution time, may lead to instability of control applications on shared platforms. Nevertheless, the security exploitation of such anomalous scenarios has not been discussed in the context of embedded control and CPSs.

The tight coupling between physical components and cyber units in control systems, makes these system vulnerable to many attacks (Cárdenas et al., 2011; Farwell and Rohozinski, 2011; Slay and Miller, 2007). These attacks are usually studied in two categories (Cárdenas et al., 2008a; Kwon et al., 2013; Lun et al., 2019). First, deceptive attacks, where the adversary interferes in the connection between sensor-controller-actuator and sends incorrect information (Amin et al., 2010; Jovanov and Pajic, 2019; Kwon et al., 2013; Liu et al., 2011; Miao et al., 2016). Second, denial-of-service attacks, where the adversary prevents sensors, controllers, or actuators from receiving data (Amin et al., 2009; Gupta et al., 2010). However, these studies do not target manipulation of the temporal properties of control applications.

Temporal attacks, on the other hand, which can affect control systems in many cases, are not well studied in the literature. Temporal attacks, if not properly mitigated, can jeopardize the stability of the control systems by introducing non-ideal timing scenarios into the control applications. The proposed temporal attacks in the literature can be classified into two categories. First, attacks that target the communication of sensors and/or actuators with the controller and result in dropping of messages that are being transferred (Fiore et al., 
2017; Moon and Başar, 2015; Shoukry et al., 2013a). Second, time delay attacks, which tamper with the temporal characteristics of the communication channel in order to delay the signals received by certain nodes (e.g., sensor, actuator, or controller) (Bianchin and Pasqualetti, 2018; Lou et al., 2019; Rahimi et al., 2015). However, in both categories, to deliver the attack the adversary changes the timing behavior of the critical messages received by the critical application. In this chapter, we introduce a different attack, in which the adversary interferes with lower criticality messages/tasks in order to affect the high criticality application.

The performance and stability of physical plants are related to the computational resources provided to their corresponding control tasks, which are running on the shared platform (Aminifar et al., 2013b; Åström and Wittenmark, 1997; Cervin, 2012). This has been extensively studied in the literature related to control scheduling co-design (Aminifar et al., 2015a,b, 2013a, 2018; Årzén et al., 2000; Behnam and Isovic, 2007; Bini and Cervin, 2008; Biondi and Buttazzo, 2018; Biondi et al., 2017; Bund and Slomka, 2013; Cervin et al., 2004; Chang et al., 2018; Dhruva et al., 2014; Fontanelli et al., 2013a; Fontanelli et al., 2013b; Fontantelli et al., 2013; Gaid et al., 2006; Goswami et al., 2012; Kumar et al., 2012; Lemmon et al., 2007; Mancuso et al., 2011; Mancuso et al., 2014; Mohaqeqi et al., 2018; Naghshtabrizi and Hespanha, 2009; Nghiem et al., 2006; Palopoli et al., 2005; Rehbinder and Sanfridson, 2000; Samii et al., 2009; Seto et al., 1996; Xu et al., 2015, 2014; Zhang et al., 2008; Ziegenbein and Hamann, 2015). Nevertheless, it is widely believed that giving more resources (e.g., percentage of available processor) to a controller leads to better control quality, and that only reducing the amount of resources represents a threat (monotonicity property). In the Vestal model (Vestal, 2007), which is widely used for the analysis of (mixed) critical systems, the implicit assumption is that exceeding a certain worst-case execution/computation time is the main threat to be considered. Thus, if a job attempts to consume more time than allowed in a certain mode (by e.g., attempting to run for a longer execution time, or more frequently), the criticality mode of the system changes (Burns and Davis, 2018). If this mode change is realized, it is assumed that the necessary isolation/protection of high criticality tasks, in the time domain, has been achieved. Similarly, in the real-time systems domain, it is assumed that providing guarantees for the case of a given upper 


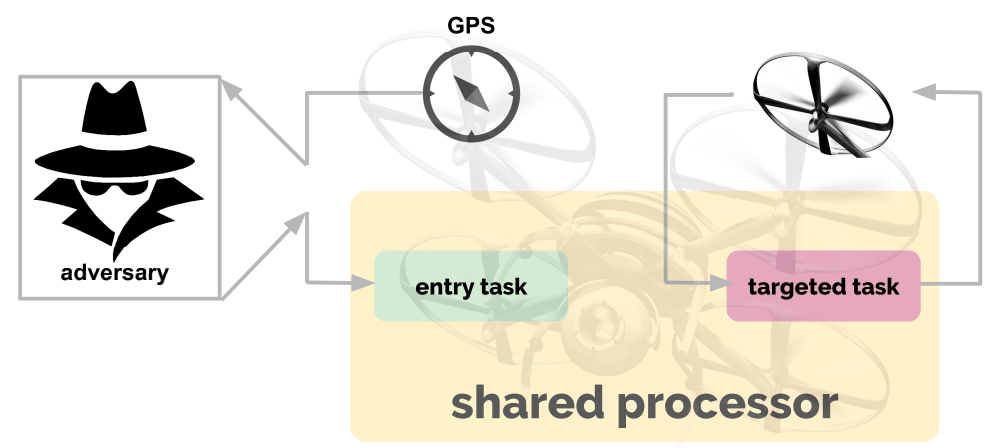

Figure 4.1: Illustration of the Butterfly attack scenario. As a result of intervention (e.g., jamming GPS signals) the entry task consumes less resources and this might lead to instability of the targeted task.

bound on execution time and a lower bound on inter-arrival time is sufficient and safe. However, as we show in this chapter, these assumptions are not sufficient to avoid security threats in the timing domain.

The attack scenario we consider in this chapter is illustrated in Figure 4.1. In this new attack scenario for CPSs, the adversary can interfere with an entry task $^{1}$ running on the shared platform (this task need not to be associated with the targeted critical application). The attacker, however, does not explicitly interfere with the execution of the targeted applications (e.g., by manipulation of registers and pointers, interfering with sensors or actuators, or similar to a replay/stealthy/denial-of-service attack). In addition, as a result of the attack, the entry task does not consume more resources (processor bandwidth/time) than the allocated ones. Instead, the attacker indirectly manipulates the usage of the resources that are provided (by providing certain inputs to a certain

\footnotetext{
${ }^{1}$ The lower criticality and less protected task that interacts with the outside world. The adversary indirectly attacks the targeted critical control application through this entry task.
} 
entry task running on the platform) in such a way that the stability of the plants associated with other (targeted) tasks is jeopardized.

Observe that, as opposed to most common attack models, here the adversary does not actively interfere with the correct execution of the targeted critical tasks/applications. The attacker will manipulate the execution of another, possibly low criticality, entry task (not the targeted application), by providing certain legitimate inputs to it. As a result of this manipulation, the entry task will consume fewer (not more) resources (time) than might be allowed.

Among the various types of temporal attacks, the attack scenario presented in this chapter exploits the observation that complex CPSs are so entangled that manipulating one entity in the system can tamper with the functionality of other entities, hence suggesting chaotic behaviors and the name Butterfly attack, after the well-known Butterfly effect..

In the next section we introduce the system model for which the attack is applicable. In Section 4.3 we give a brief background on worst-case and bestcase response times. Section 4.4 provides several examples and elaborates on the temporal behaviors the Butterfly attack exploits. Section 4.5 describes our assumptions about the environment and the capabilities of the adversary. In Section 4.6, the Butterfly attack is defined and two of its main characteristics are illustrated. Section 4.7 and Section 4.8 illustrate two real-world case studies in which the Butterfly attack results in unintended potentially devastating behavior. Finally, the mitigation and detection solutions are discussed in Section 4.9, and Section 4.10 concludes the chapter.

\subsection{System Model}

In this chapter, we assume a uniprocessor platform as described in Section 2.1 where tasks have different criticality levels and different priorities. The criticality level and priority of task $\tau_{i} \in \mathbb{T}$ is denoted by $\chi\left(\tau_{i}\right)$ and $\rho_{i}$, respectively. The set of higher priority tasks for task $\tau_{i} \in \mathbb{T}$ is denoted by $h p\left(\tau_{i}\right) \subseteq \mathbb{T}$. Task $\tau_{i} \in \mathbb{T}$ has a higher priority than task $\tau_{j} \in \mathbb{T}$ if $\rho_{i}>\rho_{j}$.

In this shared processor model, tasks need to be scheduled in such a way that they meet their deadlines and the physical plants are kept stable. We 
assume the tasks are independent, deadlines are implicit, and fixed-priority preemptive scheduling is applied. While we assume fixed-priority scheduling for the discussion in this chapter, the Butterfly attack is equally applicable in the case of other scheduling policies.

\subsection{Worst-Case and Best-Case Response Time}

Stability of a control plant is defined mathematically based on the plant model (Åström and Wittenmark, 1997). In order to measure the stability of a controller associated with a physical plant we use the standard quadratic cost metric described in Section 2.2. This metric captures the control performance and can be used to measure the stability of a control application. An infinite value of the control cost indicates that the control application is not stable (Lincoln and Cervin, 2002). For example, a quadcopter is stable if it is kept at a specific angle (equilibrium). If the angle of the quadcopter diverges from the equilibrium and the control cost for the controller to return it back is infinity, then the quadcopter is unstable.

The stability of control applications depends on the characteristics of the delays introduced into the control feedback loop. Consequently, latency and jitter are two important metrics when analyzing the stability of a periodic control application. Increased latency and jitter in the provision of of a control application's output will lead to poor control performance (increase in control cost) and can even jeopardize the stability of the physical plant. As discussed previously, when control tasks are running on a shared platform, the tasks may encounter varying delays in providing the output to the actuator in different iterations. Therefore, the physical plant, which is actuated after the controller task finishes executing, would experience unexpected varying delays. Hence, in order to be able to guarantee the stability of the physical plant, it is important to consider the impact of latency and jitter that is introduced when implementing CPSs. As discussed in Section 2.3, the worst-case jitter for a control application is calculated by Equation 2.4 and the stability 
condition is captured by Equation 2.5. By combining these two equations the stability condition for a task $\tau_{i}$ can be written as follows,

$$
\left(1-\alpha_{i}\right) R_{i}^{\mathrm{b}}+\alpha_{i} R_{i}^{\mathrm{w}} \leqslant \beta_{i}
$$

Considering the system model mentioned in the previous section, the exact worst-case response time of a task $\tau_{i}$ can be computed by the following equation (Palencia Gutierrez et al., 1998),

$$
R_{i}^{\mathrm{w}}=c_{i}^{\mathrm{w}}+\sum_{\tau_{j} \in h p\left(\tau_{i}\right)}\left\lceil\frac{R_{i}^{\mathrm{w}}}{h_{j}}\right\rceil c_{j}^{\mathrm{w}} .
$$

Similarly, the exact best-case response time of a task $\tau_{i}$ is given by the following equation (Redell and Sanfridson, 2002),

$$
R_{i}^{\mathrm{b}}=c_{i}^{\mathrm{b}}+\sum_{\tau_{j} \in h p\left(\tau_{i}\right)}\left\lceil\frac{R_{i}^{\mathrm{b}}}{h_{j}}\right\rceil c_{j}^{\mathrm{b}} .
$$

By substituting the worst-case and best-case response times from Equation 4.2 and Equation 4.3 into Equation 4.1 the stability condition for task $\tau_{i}$ is obtained. Accordingly, the stability of (the plant controlled by) task $\tau_{i}$ depends on the computation time, period, and priority of the task itself and of other tasks with higher priority than task $\tau_{i}$. The dependence of these scheduling parameters on stability is illustrated with examples in the next section.

\subsection{Scheduling Parameters and Control Performance}

In this section, we investigate the effect of scheduling parameters, i.e., sampling period, priority, and execution time, on the stability of a control application. In the following examples, we specifically show the non-monotonicity of jitter with respect to sampling period, priority, and execution time. These examples show that, counter-intuitively, providing more resources to a task does not necessarily lead to better stability results, but on the contrary, can deteriorate the performance of the control application. 
Table 4.1: Scheduling parameters of example tasks.

\begin{tabular}{|c|c|c|c|}
\hline Task & Priority & Computation Time & Period \\
\hline$\tau_{1}$ & 3 & 3 & 6 \\
$\tau_{2}$ & 2 & 2 & 8 \\
$\tau_{3}$ & 1 & 1 & 8 \\
\hline
\end{tabular}

Let us focus on a setting, wherein we assume an implicit deadline (deadline equal to the period) and a constant computation time for each task $\tau_{i}$ $\left(c_{i}=c^{\mathrm{w}}=c^{\mathrm{b}}\right)$. We consider three tasks with the characteristics described in Table 4.1, where the priorities are assigned according to Rate Monotonic (RM) scheduling. Considering a fixed-priority scheduling approach, the original schedule of these three tasks is shown in Figure 4.2a. The upward arrows show the instantiation of tasks. Note that to calculate the best-case and worst-case response times it is sufficient to consider one hyper-period. Task $\tau_{3}$ is the control task of interest in these examples and is called the targeted task in a Butterfly attack scenario. In the original task schedule, the worstcase response time for task $\tau_{3}$ is $R_{3}^{\mathrm{w}}=6$ and the best-case response time is $R_{3}^{\mathrm{b}}=4$. Therefore the latency and jitter for $\tau_{3}$ are $L_{3}=4$ and $J_{3}=2$, respectively.

\subsubsection{Changing Sampling Period}

In the first example, we investigate the effect of changing the sampling period of a task with a higher priority than the one under analysis.

Figure $4.2 \mathrm{~b}$ shows the result of a new schedule if we increase the sampling period of task $\tau_{2}$ from 8 to 24 . In this case, the worst-case response time for task $\tau_{3}$ is $R_{3}^{\mathrm{w}}=6$ and the best-case response time is $R_{3}^{\mathrm{b}}=1$. Therefore, the latency and jitter are $L_{3}=1$ and $J_{3}=5$, respectively. Comparing this to the original task schedule, in which $L_{3}=4$ and $J_{3}=2$, the latency is decreased while the jitter is increased. However, if we decrease the sampling period of task $\tau_{2}$ from 8 to 6 , based on the new schedule depicted in Figure 4.2c, the new latency and jitter are $L_{3}=2$ and $J_{3}=4$, which implies a decrease in latency and an increase in jitter compared to the original task schedule. 


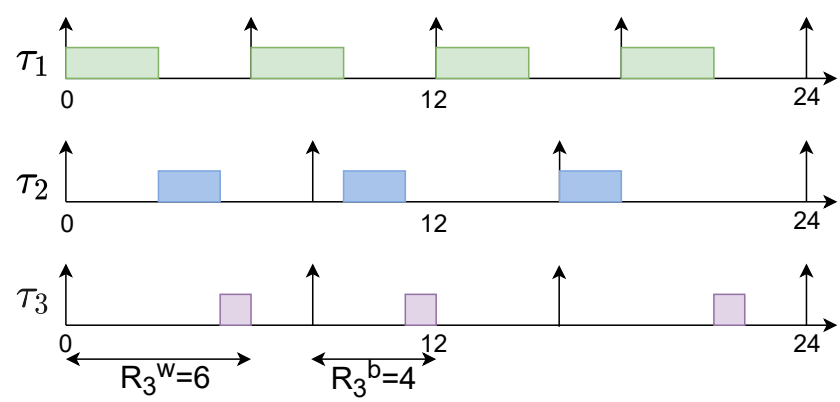

(a) Original task schedule.

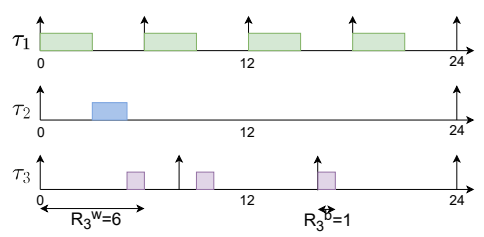

(b) Increasing period of $\tau_{2}$ to 24 .

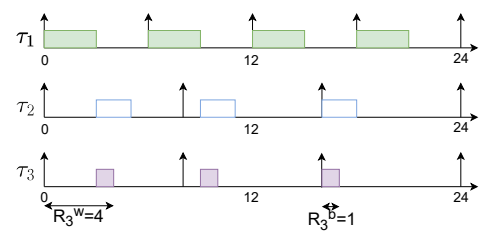

(d) Removing $\tau_{2}$.

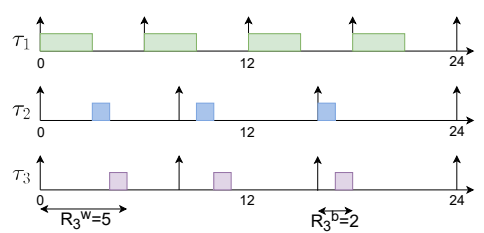

(f) Decreasing computation time of $\tau_{2}$.

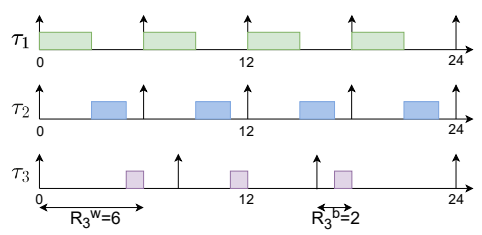

(c) Decreasing period of $\tau_{2}$ to 6 .

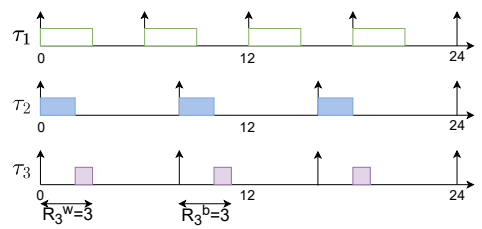

(e) Removing $\tau_{1}$.

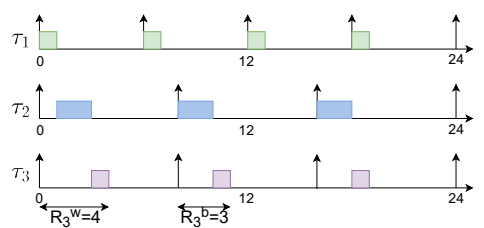

(g) Decreasing computation time of $\tau_{1}$.

Figure 4.2: Non-monotonicity of jitter w.r.t timing characteristics of other tasks. 
This means that decreasing or increasing the sampling period of a higher priority task can lead to an increase in the jitter for the task under analysis. This indicates the inter-dependency and non-monotonicity of jitter with respect to the sampling period of the higher priority tasks.

\subsubsection{Changing Priority}

In the second example, we investigate the effect of changing the priority of tasks. We do this by removing the higher priority task from the schedule (implicitly increasing the priority of the lower priority task).

First we remove task $\tau_{2}$. As a result, the worst-case response time for task $\tau_{3}$ is $R_{3}^{\mathrm{w}}=4$ and the best-case response time is $R_{3}^{\mathrm{b}}=1$. Therefore, the latency and jitter are $L_{3}=1$ and $J_{3}=3$, respectively (see Figure $4.2 \mathrm{~d}$ ). This implies a decrease in latency but an increase in jitter, compared to the original task schedule with $L_{3}=4$ and $J_{3}=2$. However, if we remove task $\tau_{1}$, according to the new schedule depicted in Figure $4.2 \mathrm{e}$, the new latency and jitter are $L_{3}=3$ and $J_{3}=0$, which implies an increase in latency but a decrease in jitter, compared to the original task schedule.

This means that increasing the priority of a task can result in an increase or a decrease in the jitter of that task. This indicates the inter-dependency and non-monotonicity of jitter with respect to priority.

\subsubsection{Changing Computation Time}

In the third example we investigate the effect of changing the computation time of a higher priority task.

First we decrease the computation time of task $\tau_{2}$ from 2 to 1 . The result is shown in Figure 4.2f. In this case, the worst-case response time for task $\tau_{3}$ is $R_{3}^{\mathrm{w}}=5$ and the best-case response time is $R_{3}^{\mathrm{b}}=2$. Therefore, latency and jitter are $L_{3}=2$ and $J_{3}=3$, respectively. Compared to the original task schedule, where $J_{3}=2$, this implies an increase in jitter. However, if we decrease the computation time of task $\tau_{1}$ from 3 to 1 , according to the new schedule depicted in Figure $4.2 \mathrm{~g}$, the new latency and jitter are $L_{3}=3$ and $J_{3}=1$, which implies a decrease in jitter, compared to the original task schedule. 
This shows that decreasing the computation time of a higher priority task can result in an increase or decrease in jitter of the task under analysis. This shows the non-monotonicity of jitter with respect to the computation time of higher priority tasks.

In the traditional analysis of mixed critical systems, the only concern for schedulability of multiple tasks sharing a processor is that a task does not exceed the assumed worst-case computation time (Vestal, 2007). Hence, the criticality mode of the system changes only when a task attempts to consume more resources than allowed in a certain mode (e.g., running for a longer computation time, or decreasing period). However, in the above examples, we showed that, due to the non-monotonicity of the jitter with respect to computation time, priority, and sampling period, consuming fewer resources than allowed can also result in abnormal timing behavior for other tasks. Finally, these examples are also valid for the case of a shared network, such as the one described in Section 2.1.2, in which a non-preemptive communication infrastructure, e.g., CAN bus is used.

To conclude this section, counter-intuitively, decreasing high-priority task interference does not necessarily lead to better response-time jitter values for the task under analysis. Therefore, the inter-dependency and non-monotonicity of jitter may lead to instability of the plant associated with the task under analysis, if not properly taken into account, and can be exploited by adversaries to carry out attacks. In the next sections we show how this abnormal timing behavior can be exploited by adversaries to destabilize a real-time control system.

\subsection{Threat Model}

We consider the following environment in the context of a Butterfly attack:

- A processing platform shared among a set of tasks $\mathbb{T}$;

- An arbitration mechanism based on which the tasks in task set $\mathbb{T}$ are scheduled at runtime;

- A safety-critical and real-time controller task $\tau_{i} \in \mathbb{T}$ that is responsible for stabilizing a physical plant $P_{i} \in \mathbb{P}$. 
We assume the following adversary in this chapter:

- The adversary may indirectly change the timing behavior of non-critical task $\tau_{j} \in \mathbb{T}$ (entry task) that shares the platform with the safety-critical controller task $\tau_{i} \in \mathbb{T}$ (targeted task).

- The adversary is aware of the non-critical (less protected) task $\tau_{j}$ (entry task) running on the shared platform with the safety-critical control task $\tau_{i}$ (targeted task).

The attack scenario presented here is considered to be indirect in the following sense:

- First, the adversary does not need to directly manipulate the shared platform, the scheduling mechanism, or the critical or non-critical tasks in task set $\mathbb{T}$ running on the shared platform.

- At the same time, the adversary only indirectly interferes with the execution of non-critical tasks. That is, the adversary uses simple techniques (e.g., jamming the input signal, providing certain inputs to the task, or delaying/advancing the incoming messages) to indirectly delay, reduce the execution time, or prevent the execution of a non-critical task $\tau_{j}$. This is possible because the execution time (as well as the execution period) of a task depends on the values of its inputs, as well as the order and timing of its inputs.

We assume that the controller task $\tau_{i}$ is protected from attacks and that the scheduler allocates a sufficient amount of resources to it. The goal of the adversary is to destabilize the physical plant $P_{i}$ by indirectly manipulating the temporal proprieties of the safety-critical task $\tau_{i}$ via the lower criticality, less protected task $\tau_{j}$. The interference with task $\tau_{j}$ may also be indirect, e.g., by jamming the input (e.g., GPS) signal, providing certain inputs to the task, or delaying/advancing the incoming messages to manipulate its temporal properties. Such interference techniques are well studied and have been performed in various real-life contexts (Davidson et al., 2016; Deligne, 2012; Drozhzhin, 2015; Highnam et al., 2016; Kerns et al., 2014; Shepard et al., 2012; Son et al., 2015). 


\subsection{Butterfly Attack}

In this section, we define the notion of the Butterfly attack. The Butterfly attack demonstrates that, contrary to popular belief (Vestal, 2007), allocating more resources to the task under analysis may jeopardize the stability of control applications and can be exploited by adversaries. In short: overprovisioning does not guarantee non-interference.

The Butterfly attack renders a task unstable by indirectly manipulating the time delay it experiences. As we discussed in Section 2.3, for a specific latency, a control application is resilient up to a certain amount of jitter. If an attacker changes the timing parameters of one or more tasks (entry tasks) such that it causes an increase in the jitter of the task under attack (targeted task), then the control application associated with the targeted task may become unstable. From Equation 4.1, we can see that jitter and, hence, stability is related to best-case and worst-case computation times, periods, and priorities of all the tasks running on the shared platform. Therefore, as the examples in Section 4.4 illustrated, changing any of these parameters can increase jitter and lead to instability of control applications.

Let us assume that a set of control applications $\boldsymbol{\Lambda}$ share a resource, e.g., processing or communication infrastructure. That is, the set of tasks $\mathbb{T}$ run on this shared platform.

Definition 4.6.1. Temporal properties of control application $\Lambda_{k} \in \boldsymbol{\Lambda}$ capture the delay patterns experienced by task $\tau_{k}$, which influence the control performance and stability of $\Lambda_{k}$. The temporal properties of task $\tau_{k}$ depend on the computation time (execution time), period, priority, release time, and offset of task $\tau_{k}$, as well as those of other tasks competing for shared resources on the same platform.

We consider tasks $\tau_{i} \in \mathbb{T}$ and $\tau_{j} \in \mathbb{T}$. We assume task $\tau_{j}$ is less critical (hence less protected) than $\tau_{i}$ i.e., $\chi\left(\tau_{j}\right) \leqslant \chi\left(\tau_{i}\right)$. The Butterfly attack on control application $\Lambda_{i}$ is defined as follows.

Definition 4.6.2. Indirect manipulation of the temporal properties of a less critical task $\tau_{j}$ to modify the schedule of a more critical task $\tau_{i}$ such that the 
application $\Lambda_{i}\left(\tau_{i}\right.$ relates to application $\left.\Lambda_{i}\right)$ slips out of its expected behavior, is referred to as Butterfly attack.

Such temporal manipulations may be adopted by adversaries to deteriorate the performance of control applications or even to destabilize the physical plant $P_{i}$ controlled by task $\tau_{i}$.

The Butterfly attack exploits two important characteristics of control tasks that are utilizing a shared platform. These two characteristics make the Butterfly attack undetectable and effective (see Section 4.7 and Section 4.8):

1. Inter-dependency: The computation time, priority and period of tasks are considered independent. Nevertheless, since the tasks run on a shared platform, the temporal properties and, in turn, the stability of the tasks depend on each other. In fact, changing the parameters (computation time, period, and/or priority) of one task may alter the temporal properties of other tasks. Therefore, an attacker might interfere with the parameters of one (less critical/protected) task, in order to manipulate the temporal properties of another (more critical/protected) task and make it unstable.

2. Non-monotonicity: Temporal properties (and hence stability) of a physical plant are related to the resources provided to its control task. Decreasing the amount of resources for a task will decrease the performance of the controller and may jeopardize the stability of its physical plant. However, the relation between the real-time parameters and the temporal properties of tasks is not necessarily monotonic. Specifically, as shown in Section 4.4, increasing the resources (e.g., processor share) available to a task may increase the jitter and, hence, reduce the performance of the controller or even jeopardize the stability of the physical plant. Therefore, counter-intuitively, an attacker can destabilize a task by increasing the resources that are available to that task.

Note that because of the two aforementioned characteristics of the Butterfly attack, it is not trivial to detect and mitigate it. System designers try to keep a critical task stable by protecting only that task from attackers and making sure that the task has sufficient resources to execute (Vestal, 2007). 
The Butterfly attack demonstrates that this is not enough. First of all, the attacker renders a control application unstable without interfering with the timing parameters of the task under attack, but rather with other non-critical (potentially less protected) tasks. Second, the attacker does not necessarily need to reduce the amount of resources allocated to a task in order to make it unstable, but instead can make a task unstable by increasing the resources available to the task under attack (by, e.g., decreasing the amount of resources reserved for other tasks).

In the next sections, we will demonstrate how the Butterfly attack can be applied, based on the inter-dependency and non-monotonicity present in complex real-time control and CPSs.

\subsection{Case Study 1: Automotive Application}

Attacks against modern automobiles have gained a lot of attention (Koscher et al., 2010; Miller and Valasek, 2015), mainly due to the critical nature of such systems. Miller and Valasek (Miller and Valasek, 2015) demonstrated a remote attack against commercial automobiles (Drozhzhin, 2015). The goal of this attack is to remotely control critical tasks, e.g., control steering wheel, engine, transmission, and braking system, which communicate over a CAN bus. This attack exploits vulnerabilities in the entertainment system of a car through a Wi-Fi connection. However, the multimedia system is not connected to the CAN bus directly. The isolation, or so-called air-gap, between the entertainment and critical functionality in vehicles is intended to reduce the possibility of attack. Nevertheless, while the multimedia system cannot directly connect to the CAN bus, it can still communicate with another component that is connected to the CAN bus, i.e., the V850 controller. An attacker could discover an opportunity to change the firmware of the V850 controller (Eltze, 1997) and take control over it. The V850 processor is responsible for interacting with the CAN buses. Once the attacker has access to the V850 controller, it is possible to temporarily stop a message by forcing a collision between the target Electronic Control Unit (ECU) and the attacker-controlled ECU. 
The Miller and Valasek (Miller and Valasek, 2015) attack shows that, in practice, it is possible to take over CAN communication channels inside a car remotely. Direct manipulation of the critical messages and applications can be easily/quickly identified and can be counteracted by the existing intrusion detection/mitigation mechanisms (Karray et al., 2018; Marchetti and Stabili, 2017; Stabili et al., 2017; Taylor et al., 2015).

In the Butterfly attack, however, we assume that an adversary is more restricted and, at the same time, more stealthy. We assume that the adversary can only temporarily prevent some non-critical CAN messages from using the CAN bus. The Butterfly attack provides the opportunity to target highcriticality applications by manipulating less protected, low-criticality applications. The attack shows that an adversary may delay messages of a noncritical and less sensitive application, leading to an increase in the period of its task, thereby increasing the jitter experienced by a high-criticality task and application (see Section 4.4.1). As a result of such an attack, the highcriticality control application may become unstable due to this increase in jitter, as discussed in Section 2.3.

In the following we show the effectiveness of the Butterfly attack by launching it against a DC motor control application operating inside an electric vehicle. Our case study considers a subsystem consisting of a highly critical application that controls a DC motor in an electric vehicle. The ECU hosting the DC motor control application also runs several other applications: propulsion control software, cruise control, and a high-precision vehicle positioning algorithm. These control applications all receive inputs from multiple sensors and ECUs communicating over a shared CAN bus. These inputs include GPS position from a telematics ECU, wheel speed sensors, and an inertial measurement unit for pitch, roll, and yaw rate. Hosting a large variety of functions on a single ECU is a current trend in the automotive industry, often referred to as ECU consolidation or up-integration (Maul et al., 2018; Weiss et al., 2016). While cruise control and vehicle positioning are important functions, these are less critical than the control of the vehicle's DC motor. Therefore, we consider that the CAN messages of the DC motor control are protected, and that the adversary is only able to block CAN messages used by the less critical tasks. 
Let us consider a DC motor setting proposed in (Messner and Tilbury, 2017b). The mathematical model of the plant is provided in the following differential equation,

$$
\dot{\boldsymbol{x}}=\boldsymbol{A x}+\boldsymbol{B u},
$$

where $\boldsymbol{x}$ and $\boldsymbol{u}$ are the state of the DC motor (rotational speed of the rotor and the armature current) and input signal (armature voltage), respectively. The matrices $\boldsymbol{A}$ and $\boldsymbol{B}$ are extracted based on the physics of the DC motor as follows,

$$
\boldsymbol{A}=\left[\begin{array}{cc}
-11 & 0 \\
1 & 0
\end{array}\right], \quad \boldsymbol{B}=\left[\begin{array}{c}
32 \\
0
\end{array}\right]
$$

Considering this model and using the jitter margin toolbox, the stability curve is depicted in Figure 4.4 and the stability condition is derived from this curve.

We assume that the sensor is periodically sending messages over CAN to the DC motor controller. The controller task is receiving its input from the CAN bus and a job is activated after receiving the input. The CAN bus is shared among several other control applications, i.e., propulsion control software, cruise control, and a high-precision vehicle positioning algorithm. Let us focus only on the high-precision vehicle positioning and DC motor controller tasks using the CAN bus to communicate. The two applications have the following parameters,

$$
\begin{array}{ll}
\tau_{1}: & \rho_{1}=2, c_{1}=10, h_{1}=15, \\
\tau_{2}: & \rho_{2}=1, c_{2}=5, h_{2}=30,
\end{array}
$$

where task $\tau_{2}$ is the task that is running the $D C$ motor controller to control the rotational speed of its rotor and is the target task for the adversary, while task $\tau_{1}$ is the high-precision vehicle positioning task with a lower criticality. CAN messages are scheduled using the non-preemptive fixed-priority scheduling algorithm. Priorities are assigned according to RM scheduling.

To simulate the shared platform, we use the Truetime toolbox (Cervin et al., 2003) to provide the scheduling of the tasks. TrueTime is a MATLAB/Simulink-based simulator for real-time control systems, which facilitates co-simulation of controller task execution in real-time kernels. If the 


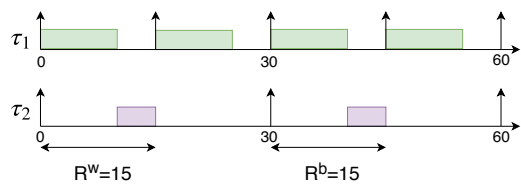

(a) Before increasing the period of task $\tau_{1}$.

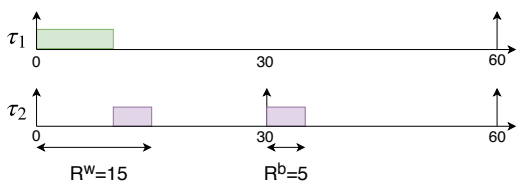

(b) After increasing the period of task $\tau_{1}$ from 15 to 60 .

Figure 4.3: Schedule of vehicle positioning and DC motor controller tasks on the shared platform. The green (vehicle positioning) task has a higher priority than the red (DC motor controller) task. The properties of the tasks are $\tau_{1}=(2,10,15), \tau_{2}=(1,5,30)$.

fixed-priority preemptive scheduling policy is adopted for the tasks, then the tasks execute according to the schedule represented in Figure 4.3a. As a result, the latency and jitter for the DC motor controller task would be $15 \mathrm{~ms}$ and $0 \mathrm{~ms}$, respectively. This latency and jitter are shown by a blue star in Figure 4.4 and the point falls within the stable area. Therefore, the DC motor is guaranteed to be stable with this schedule, and this is also observed in our experiment. In our experiment, using our control-scheduling co-simulation framework, we change the desired rotor speed at time 1s, and the rotor speed converges to the desired speed after some oscillation (see Figure 4.5a) and the control cost is kept finite (see Figure 4.5b), hence the system remains stable.

In order to perform the Butterfly attack, we run the same simulation and change the desired rotor speed at time 1s. However, this time, the adversary increases the period of the messages for task $\tau_{1}$, i.e., high-precision vehicle positioning task, from $15 \mathrm{~ms}$ to $60 \mathrm{~ms}$ (e.g., by temporarily interrupting CAN messages from the GPS sensor). Note that by increasing the period of the messages triggering $\tau_{1}$, we increase the activation period of the task and, thus, increase the available processor share for the $D C$ motor task. Intuitively, this should lead to better control performance in the DC motor. However, Figure $4.3 \mathrm{~b}$ shows that the latency and jitter of $\tau_{2}$ is $5 \mathrm{~ms}$ and $10 \mathrm{~ms}$, respectively. This set of latency and jitter values is shown with a red plus in Figure 4.4, and the point falls within the unstable area. Therefore, the DC motor is not guaranteed to be stable with these latency and jitter values. 


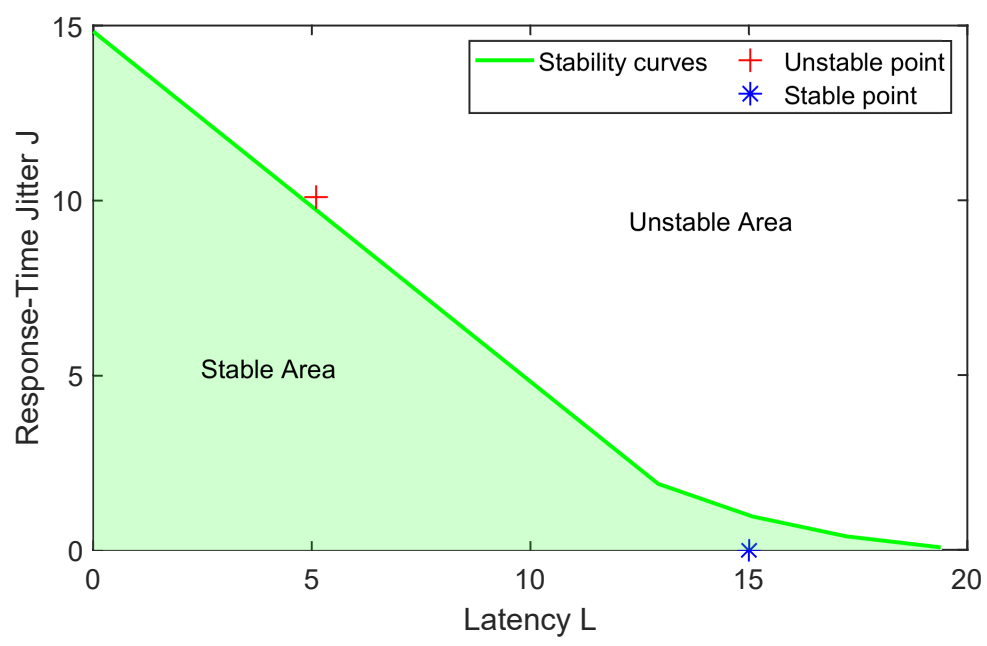

Figure 4.4: The stability curve generated by Jitter Margin (the area below the curve is the stable area). The shaded green region is the area in which the plant is guaranteed to be stable. The blue star (latency $L=15$ and jitter $J=0$ ) shows a stable point and the red plus (latency $L=5$ and jitter $J=10$ ) shows an unstable point.

This is also confirmed in our control-scheduling co-simulation framework. Figure 4.6a shows that the controller is not able to converge the rotor speed into the desired speed and the control cost goes to infinity (see Figure 4.6b), which indicates that the plant has become unstable.

\subsection{Case Study 2: Unmanned Aerial Vehicle (UAV)}

Unmanned Aerial Vehicles (UAVs) have autopilot software that is a real-time system that requires rapid responses to changing sensor data to keep the UAV under control. In this attack scenario, we consider ArduPilot (ArduPilot Team, 2021), which is open source autopilot software. Many UAV companies 


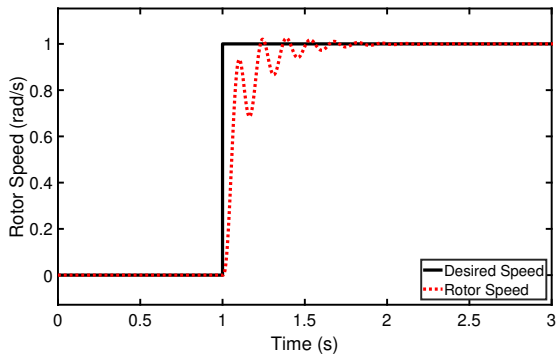

(a) DC motor rotational speed (stable).

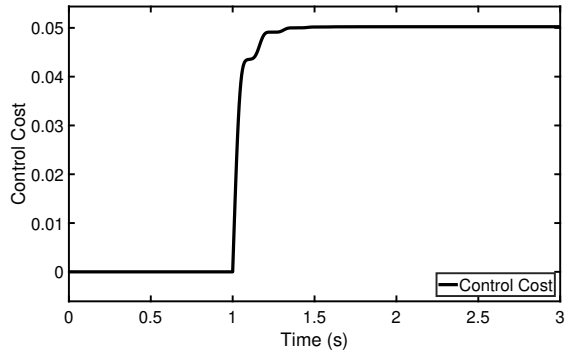

(b) DC motor control cost (stable).

Figure 4.5: Stable DC motor without adversary.

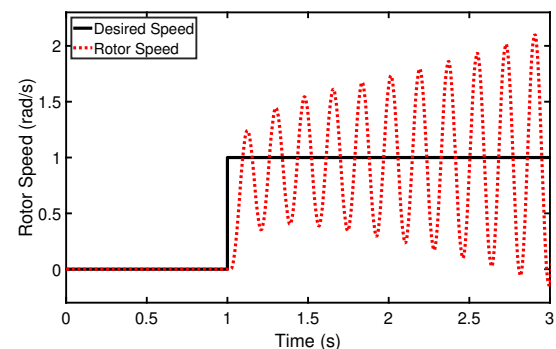

(a) DC motor rotational speed (unstable).

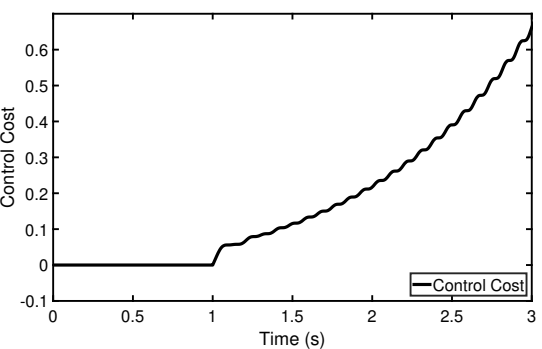

(b) DC motor control cost (unstable).

Figure 4.6: Unstable DC motor in the presence of adversary.

(e.g., 3DR, jDrones, PrecisionHawk, AgEagle, and Kespry) use ArduPilot on their devices and large corporations (e.g., NASA, Intel, and Insitu/Boeing) use it for testing and development.

ArduCopter (a version of ArduPilot customized for copters) has 19 main realtime tasks with deadline constraints. These tasks run under the context of a flight controller process (main_loop) and are scheduled using a real-time scheduling system. Each real-time task handles a specific flight control operation and executes a loop that has two real-time parameters: a period and a deadline. In this case-study, we consider two of the tasks running in the controller process: update_GPS and run_nav_updates. The update_GPS task reads the GPS signal. The run_nav_updates task is a critical task that runs 
the autopilot task and actuates commands to the servos to control the UAV. Here, we consider the scenario in which the adversary manipulates the timing of a less critical task like update_GPS, which will lead to an increase in jitter for the more critical run_nav_updates task.

The update_GPS function (see Source Code 4.1) starts with a condition that, if not satisfied, will prevent the main part of the code from executing. The condition checks if a new message has been received and if the status of the received signal satisfies previous assumptions. Therefore, if an attacker jams the GPS signal, then the entire update_GPS task will have a much smaller and negligible (close to 0) execution/computation time.

\section{Source Code 4.1: update_GPS}

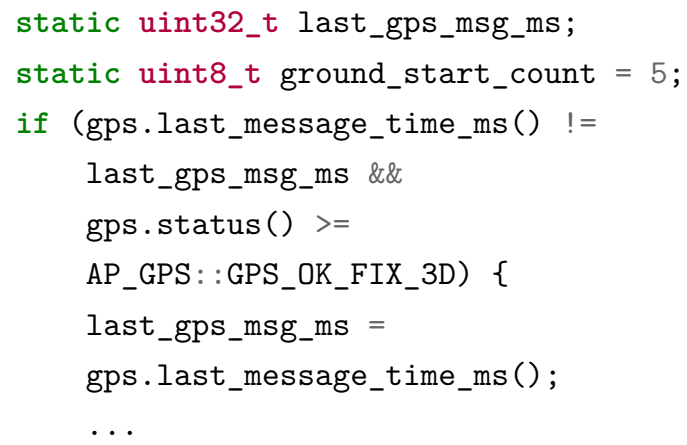

Reducing the computation time of the update_GPS task can cause intolerable jitter for other tasks running on the same microprocessor (e.g., run_nav_updates). Note that in this scenario the attacker only decreases the computation time of update_GPS, which intuitively should be harmless to other tasks and, therefore, is not prevented by the scheduler. However, it will create large jitter values for other tasks and may lead to instability of the UAV.

We consider a Butterfly attack scenario on a quadcopter running the flight control software and show experimentally how the attack would render the quadcopter unstable. We assume that the flight control software is running on the quadcopter's processor. Therefore, all real-time tasks are scheduled on the shared processor. We focus only on the update_GPS and run_nav_updates tasks running on the processor. Let's consider that the two tasks have the 


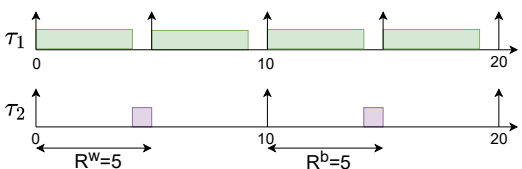

(a) Before increasing the period of task $\tau_{1}$.

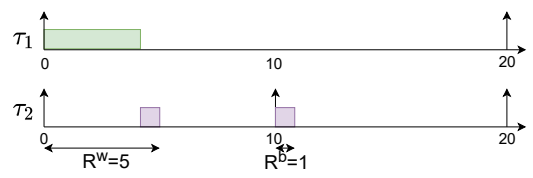

(b) After increasing the period of task $\tau_{1}$ from 5 to 20 .

Figure 4.7: Schedule of update_GPS and run_nav_updates tasks on the shared platform. The green (update_GPS) task has a higher priority than the red (run_nav_updates) task. The properties of the tasks are $\tau_{1}=(2,4,5), \tau_{2}=(1,1,10)$.

following parameters,

$$
\begin{array}{ll}
\tau_{1}: & \rho_{1}=2, c_{1}=4, h_{1}=5, \\
\tau_{2}: & \rho_{2}=1, c_{2}=1, h_{2}=10,
\end{array}
$$

where task $\tau_{2}$ is the task that is running the controller (run_nav_updates) to stabilize the quadcopter in the desired position and task $\tau_{1}$ is the update_GPS task. Priorities are assigned according to RM scheduling.

We use the model provided in (Bouabdallah, 2007) to simulate the quadcopter in Simulink MATLAB. The model is linearized and can be written in statespace form as follows,

$$
\dot{x}=A x+B u,
$$

where $\boldsymbol{x}$ is the state of the quadcopter and shows its position and angle, and $\boldsymbol{u}$ is the input signal to the quadcopter. The matrices $\boldsymbol{A}$ and $\boldsymbol{B}$ are calculated using the dynamic model of the quadcopter and depend on its physical properties, e.g., inertia moments in each axis, rotor inertia, propeller distance from center, and overall mass. The input signal is a vector of four elements (one for each rotor) and is calculated based on the current state of the quadcopter, such that the quadcopter is positioned at a desired coordinate and angle. The controller task receives the current and desired state of the quadcopter in each period and provides input signals to position the quadcopter at the desired coordinate. 


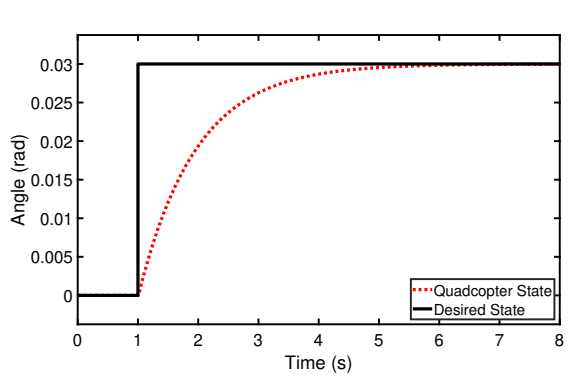

(a) Quadcopter vertical angle (stable).

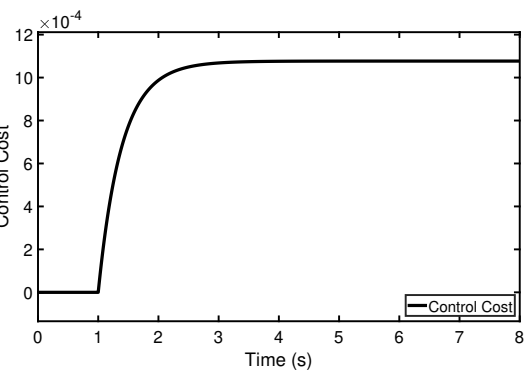

(b) Quadcopter control cost (stable).

Figure 4.8: Stable quadcopter without adversary.
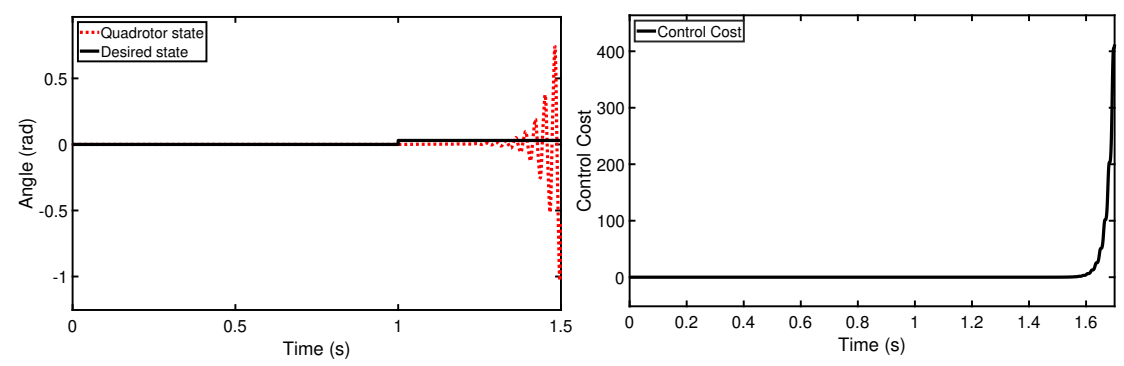

(a) Quadcopter vertical angle (unstable).

(b) Quadcopter control cost (unstable).

Figure 4.9: Unstable quadcopter in the presence of adversary.

If fixed-priority RM scheduling is applied, the tasks execute according to the schedule represented in Figure 4.7a. As a result, the run_nav_updates task experiences latency and jitter equal to $5 \mathrm{~ms}$ and $0 \mathrm{~ms}$, respectively. With these values for latency and jitter, the quadcopter is stable. In our controlscheduling co-simulation framework, at time 1s we change the desired states (the horizontal angles) of the quadcopter. The controller manages to converge to the desired state (see Figure 4.8a) and the control cost is kept finite (see Figure $4.8 \mathrm{~b}$ ), which indicates the stability of the system.

To perform the Butterfly attack, we run the same simulation and change the desired state of the quadcopter at time $1 \mathrm{~s}$ in our control-scheduling cosimulation framework. However, this time, the attacker increases the period of the update_GPS $\left(\tau_{1}\right)$ task from $5 \mathrm{~ms}$ to $20 \mathrm{~ms}$. This is performed by jam- 
ming the GPS signal such that three out of four messages are not received. Note that, increasing the period of $\tau_{1}$, reduces the interference to which $\tau_{2}$ is subject, and hence the available processor share for $\tau_{2}$ is increased. Intuitively, this should lead to better control performance of run_nav_updates $\left(\tau_{2}\right)$. Nevertheless, according to the new schedule for $\tau_{1}$ and $\tau_{2}$ depicted in Figure $4.7 \mathrm{~b}$, the latency of $\tau_{2}$ decreases to $1 \mathrm{~ms}$ but the jitter increases to $4 \mathrm{~ms}$. The quadcopter is not stable with this latency and jitter. After changing the desired state, the controller is unable to converge the state of the quadcopter into the desired state (see Figure 4.9a) and the control cost tends to infinity (see Figure 4.9b), which indicates that the system has become unstable.

The above example shows how a Butterfly attack can destabilize a UAV. In the following we show that, using this attack, an adversary is also able to hijack the UAV and move it to a different location. In the quadcopter model, the autopilot software changes the desired angles of the quadcopter to move it toward a desired location. We experimentally show that if the adversary interferes during this transition process and introduces a specific amount of jitter, then she is able to change the location of the quadcopter.

We again focus on two tasks running on the processor, with the parameters stated in Equation 4.7. Let us assume that the autopilot software changes the desired angle of the quadcopter at time 1s. As a result, the quadcopter will end up in position $(X=17, Y=17$, and $Z=5.5)$ at time 4 s. Figure 4.10 shows the location of the quadcopter during this transition when there is no attack and the quadcopter reaches the desired location at time 4s. Figure 4.12 shows the path (blue line) the queadcopter follows to reach the destination.

In order to apply the Butterfly attack, we simulate an adversary that periodically increases the period of the update_GPS task from 5 to 20 and decreases it back to 5 (e.g., by jamming 3 out of every 4 GPS messages). This process is repeated 50 times during the transition. Subsequently, the quadcopter does not become unstable, but it follows an unexpected path (red line in Figure 4.12) and at some point during the attack it enters an unsafe zone, which is far from the point where the quadcopter is supposed to be, and where the quadcopter can be hijacked. After the attack, the quadcopter again starts to converge to the desired location that the autopilot software has specified. Figure 4.11 shows the location of the quadcopter during the transition time. 


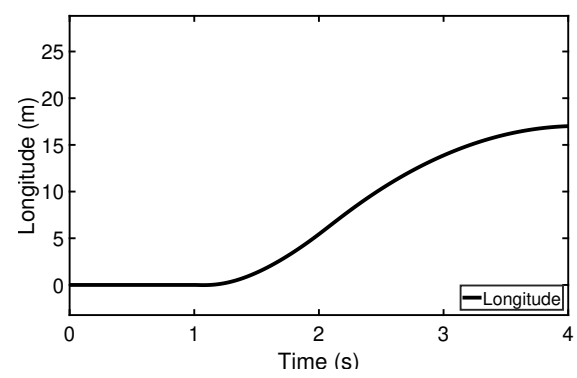

(a) Longitude

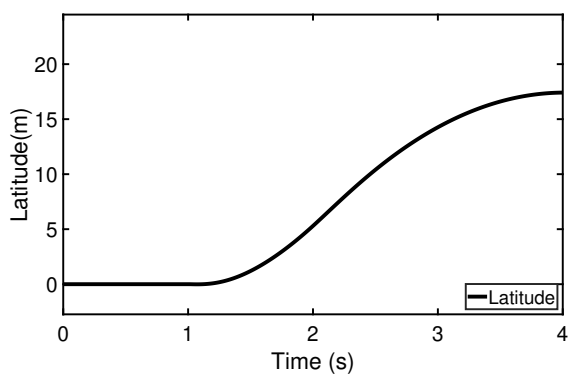

(b) Latitude

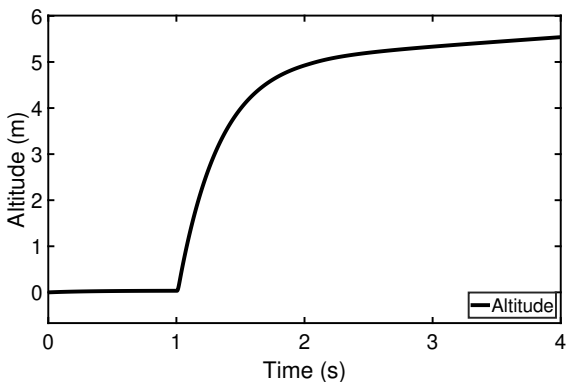

(c) Altitude

Figure 4.10: Position of quadcopter during transition.

Note that the adversary has some control over the location of the quadcopter during this attack. Therefore, the adversary can direct the quadcopter into a desired zone by imposing a certain amount of jitter. Figure 4.12 shows the two-dimensional position of the quadcopter over time, with and without the Butterfly attack. 


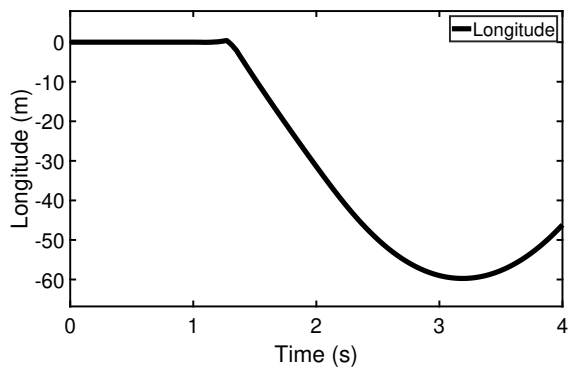

(a) Longitude

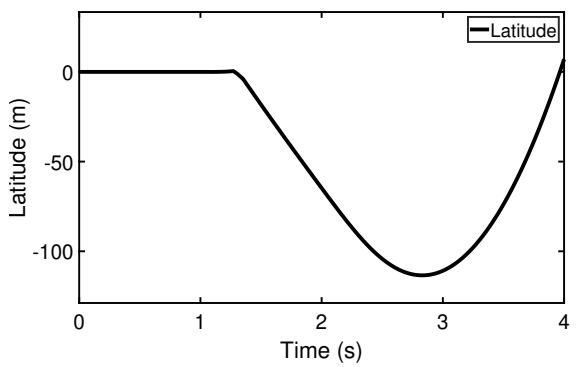

(b) Latitude

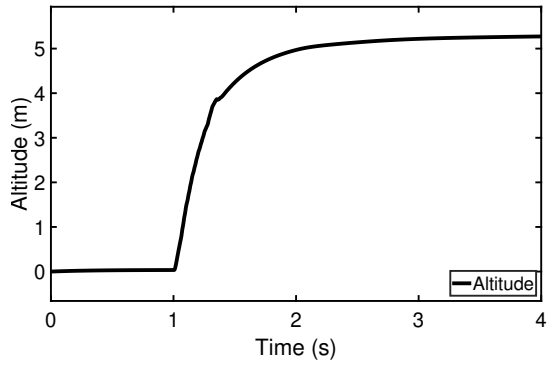

(c) Altitude

Figure 4.11: Position of quadcopter in the presence of an adversary.

\subsection{Discussion}

In this section, we discuss several possible mitigation, detection, and defense mechanisms against Butterfly attacks. To detect and mitigate a Butterfly attack, it is possible to synthesize a set of safety constraints during the design phase. This is similar to monitoring overruns in current operating systems, 


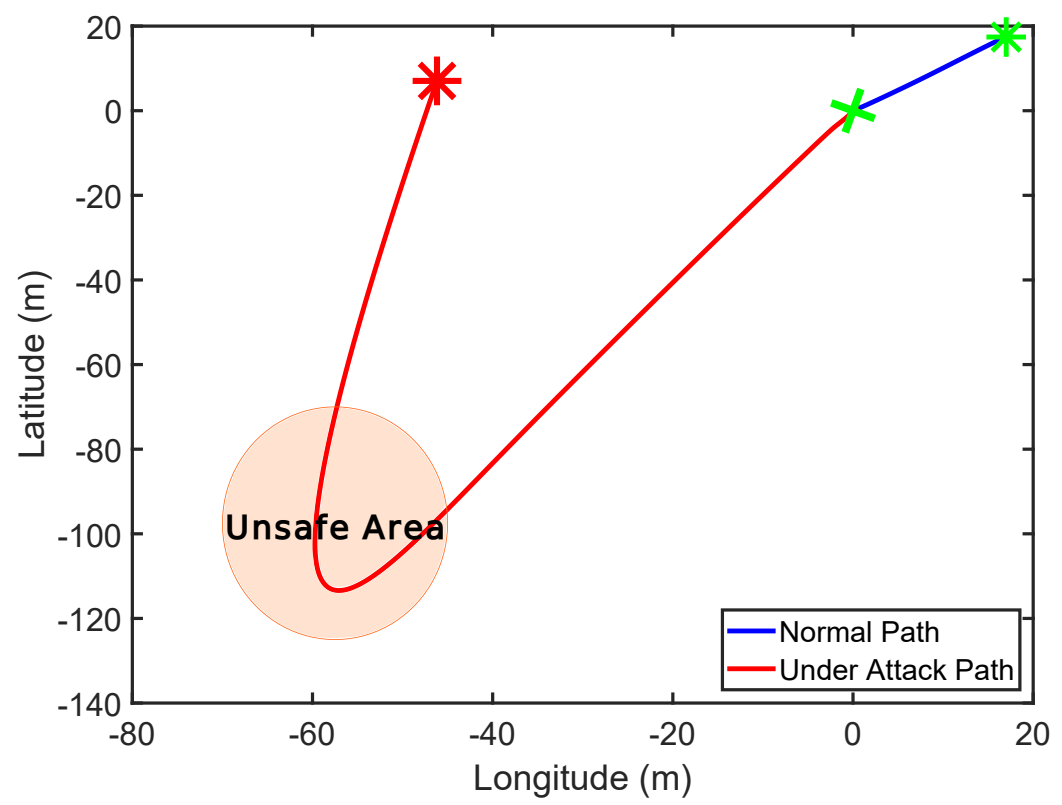

Figure 4.12: Two-dimensional position of the quadcopter between time $0 \mathrm{~s}$ and $4 \mathrm{~s}$. The cross and star signs in green show the initial position $(0,0)$ and desired destination $(17,17)$ of the quadcopter, respectively. The blue line shows the path of the quadcopter when there is no attack and the red line shows its path when an attack is ongoing. The red star sign at the end of red path shows the position of the quadcopter after $4 \mathrm{~s}$, when it is under attack.

but captures more temporal aspects of the safety-critical applications, including the delay, latency, and jitter. Then, at runtime, these constraints will be monitored by an intrusion detection unit to identify potential threats and initiate the mitigation protocol.

The mitigation protocol may involve executing a dummy task to compensate for the timing manipulation introduced into the system by the adversary and, e.g., compensating for increased period or decreased execution time of a lower criticality task. In this approach, however, the resources are not used for running applications during some periods of time. One solution could instead be to promote low-priority tasks in order to avoid this phenomenon. Alter- 
natively, the mitigation protocol might initiate a procedure to switch to more robust controllers with, of course, worse control performance, for the safetycritical applications. This mitigation protocol requires careful planning for the switching procedure, due to the potentially pathological scenarios associated with switching control. In particular, it is well-known that switching between two stable controllers may still destabilize the control plant (Hespanha and Morse, 2002) if not done carefully.

Another mitigation technique is to assign priorities with respect to task criticality. By doing this the adversary is not able to interfere with high-criticality tasks by manipulating less-critical ones. Nonetheless, this apparently simple solution is often not applicable: (1) In many cases the system designer needs to assign higher priorities to less-critical tasks for schedulability reasons so that the less critical tasks can meet their deadlines. (2) This mitigation technique can only be applied to systems that use fixed-priority scheduling, while the Butterfly attack is also applicable to other scheduling algorithms like EDF (Earliest Deadline First).

In the context of control design, mitigation might be provided by designing a controller that is robust to temporal attacks (Pajic et al., 2014; Shoukry et al., 2013a). However, this might result in more complex and resource-consuming controllers with potentially lower control quality. Essentially, this is because such controllers are designed for a scenario that does not correspond to the expected average-case scenario.

The ultimate solution to eliminating the possibility of such temporal attacks is to ensure temporal isolation using bandwidth allocation and control servers (Aminifar et al., 2015a; Cervin and Eker, 2003). The main drawback of control servers is either lack of any hard guarantees or over-provisioning resource allocation, which may leave resources under-utilized, even as low as $50 \%$ of the total capacity. To address this issue, several online resource allocation mechanisms have been proposed in the literature (Aminifar et al., 2015b). Therefore, a comprehensive study of mitigation and defense mechanisms for complex CPSs remains the topic of our future work. 


\subsection{Conclusion}

In this chapter, we introduce the Butterfly attack, a new attack scenario for CPSs. The Butterfly attack highlights the importance of the implementation aspects of CPSs on the underlying execution platforms and the temporal sensitivity of control applications implemented on shared platforms. Such resource sharing may lead to complex timing behaviors and, in turn, counterintuitive timing anomalies that can be exploited by an adversary to render a critical control system unstable. We demonstrate the possibility of such attacks in two case-studies from the automotive and avionic domains, and discuss briefly several methods to mitigate them. 


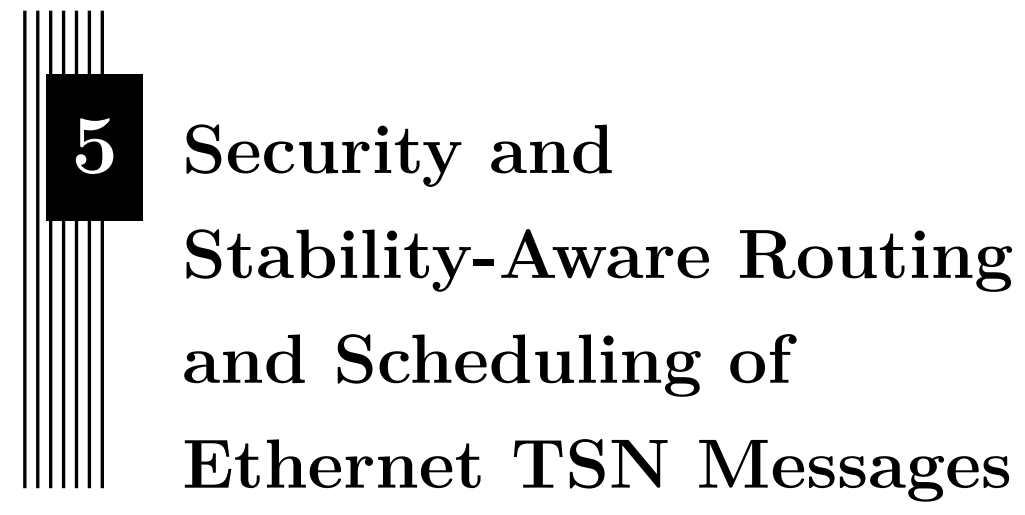

Real-time communication over Ethernet is becoming important in various application areas of cyber-physical systems, such as industrial automation and control, avionics, and automotive networking. Since such applications are typically time critical, Ethernet technology has been enhanced to support time-driven communication through the IEEE 802.1 TSN standards. The performance and stability of control applications are strongly impacted by the timing of the network communication. Thus, in order to guarantee stability requirements, when synthesizing the communication schedule and routing, it is necessary to consider the degree to which control applications can tolerate message delay and jitter. These networked control applications need to be resilient against malicious interference and hence, the proposed scheduling and routing technique that guarantees stability for control applications must be security aware. The aim in this chapter is to answer RQ3 (see Section 1.2). In fact, we propose a method for jointly solving the message scheduling and routing problem for networked CPSs based on the time-triggered Ethernet TSN standards. We consider this communication synthesis problem in the 
context of control applications and guarantee their worst-case stability, explicitly taking security requirements into consideration.

Considering the inherent complexity of the network communication synthesis problem, we also propose new heuristics in this chapter to improve synthesis efficiency without any major loss of quality. Our experiments demonstrate the effectiveness of the proposed solutions. The content of this chapter is based on the following publications: (Mahfouzi et al., 2019a, 2018).

\subsection{Introduction and Related Work}

Reliability and security are two major challenges in the Internet of Things (IoT) and CPS domains (Thiele, 2017; Weber, 2010). On the one hand, reliability, particularly in terms of timing guarantees and real-time operation, is absolutely essential for many applications, including control and real-time systems. Failure to provide such timing guarantees could lead to catastrophic incidents involving human lives. On the other hand, security, has become important for many safety-critical applications to ensure safety in the presence of adversarial actions. As discussed in Chapter 1, in the case of IoT and CPS applications, the resilience to adversarial attacks is essential for two main reasons: (1) the cyber elements of the CPSs and their increased connectivity through various wired and wireless communication technologies increase the opportunities for an adversary to compromise the safety of the system, and (2) the physical elements of the CPSs, if they are of a safety-critical nature, make it possible for an adversary to create a catastrophic incident that could be even worse than traditional information security threats. The criticality of attacks on CPSs has already been demonstrated for both nuclear facilities and automotive systems (Farwell and Rohozinski, 2011; Miller and Valasek, 2015; Shoukry et al., 2013b). Due to lack of authentication and encryption mechanisms, specifically in automotive industry (Bello et al., 2018), it is easy for an attacker to impersonate or sniff transmitted messages. As mentioned before, authors in (Miller and Valasek, 2015) describe a concrete attack on the communication network in a car. They show how an adversary can impersonate sensors. In their attack, the adversary is able to manipulate messages transmitted through the CAN bus to the brake system and apply the brakes on behalf of the driver. Attacks of this type, which target critical physical 
plants, can jeopardize human life if not taken into consideration. In this chapter, we specifically consider man-in-the-middle attacks that impersonate or sniff critical control messages in CPSs. Such an attack can be prevented by appropriate authentication and encryption measures.

We noted in Chapter 1 that, in addition to the challenges discussed above, CPSs are often highly constrained in terms of resources, i.e., computational power, communication bandwidth, storage space, and battery lifetime. Therefore, designing reliable and secure CPSs becomes more challenging and requires careful allocation and optimization of available resources. In particular, there is an inherent trade-off between security strength and available resources (Kocher et al., 2004; Ravi et al., 2004). That is, the higher the security requirements, the more resources should be allocated for that purpose.

Due to growing bandwidth requirements, real-time communication over Ethernet is of increasing importance in embedded control and CPSs. Application domains include industrial automation and control, avionics, and active safety and automated driving applications (Bello, 2011; Chakraborty et al., 2016; Samii and Zinner, 2018). Several time-driven approaches like 802.1Qbv (IEEE Standard, 2015), developed by the IEEE 802.1 Time-Sensitive Networking (TSN) task group, and TTEthernet (Steiner et al., 2018) exist to enhance standard Ethernet with real-time properties such as time determinism and packet delivery guarantees. The temporal properties of an Ethernet network depend significantly on the schedules and routes of the data flows along switches. Researchers have recently studied various design-space exploration problems to synthesize Ethernet schedules and routes in the context of hard deadlines and worst-case latencies. While this model is applicable to some real-time applications, it does not take into consideration temporal properties like average delay and jitter, which as discussed in the previous chapter, are known to impact control performance and stability in CPSs (Aminifar et al., 2015b, 2018; Cervin, 2012; Deng et al., 2016; Lincoln and Cervin, 2002; Quaglia et al., 2012; Samii et al., 2009).

In this chapter, we first propose a stability-aware routing and scheduling methodology for control applications that communicate over Ethernet networks. Then we build on this routing and scheduling method and target two main challenges of the IoT and CPS domains, i.e., maximizing the resilience 
and security of the system against adversarial attacks, while satisfying timingrelated constraints.

In the context of TTEthernet networks with multiple hops, Steiner (Steiner, 2010) proposed an approach based on Satisfiability Modulo Theory (SMT) to address the problem of schedule synthesis. This approach has been adapted in (Craciunas et al., 2016) to support the recent IEEE 802.1Q amendment for scheduled traffic (IEEE 802.1Qbv). Time-triggered schedule synthesis has recently been extended in various directions, such as bandwidth optimization for best-effort traffic (Tămaş-Selicean et al., 2015), mixed-criticality systems (Steiner, 2011), resilience to link failures (Avni et al., 2016), traffic class assignment (Gavrilut and Pop, 2016), and modeling of time-synchronization precision (Zhang et al., 2014).

Tamas-Selicean et al. (Tămaş-Selicean et al., 2015) considered scheduling, frame packing, and route selection separately in a Tabu Search heuristic. The proposed routing strategy increases delay and jitter of time-triggered frames so as to meet their deadlines with as little time slack as possible, in order to increase schedulability and bandwidth, respectively, for rate-constrained and best-effort communication. The approach is customized to rate-constrained and best-effort traffic and is neither applicable nor generalizable to timetriggered Ethernet communication for hard real-time or control applications. Pop et al. (Pop et al., 2016) presented a design optimization framework for Ethernet with time-triggered (TT) and Audio Video Bridging (AVB) traffic classes, with routing fixed and given by the shortest path for each TT data flow, leaving the entire TT routing optimization problem unsolved. Smirnov et al. (Smirnov et al., 2017) presented a 0-1 Integer Linear Programming (ILP) formulation for routing and scheduling, where the network model is limited to bus-based, half-duplex communication with gateways. Their solution is limited to synthesis of collision-free schedules without any deadline constraints, and it merely determines whether or not pairs of messages share the same link.

The security of real-time applications under resource constraints, e.g., in the automotive domain (Sagstetter et al., 2013), has also been discussed in several studies over the past decade (Aminifar et al., 2017; Jiang et al., 2011, 2012, 2013; Kang and Son, 2006; Lin et al., 2009; Xie and Qin, 2007). In (Kang 
and Son, 2006), authors propose a lightweight heuristic to optimize the cryptographic key length under real-time constraints. Xie and Qin (Xie and Qin, 2007) propose an online algorithm for scheduling periodic tasks under security and timing constraints, which tries to maximize a weighted sum of the individual security values, a metric defined by the authors. Similarly, Lin et al. (Lin et al., 2009), perform optimization for the security of real-time systems, based on an ILP formulation and a heuristic.

More recently, Jiang et al. (Jiang et al., 2011, 2012) used constraint logic programming to optimize message encryption under real-time constraints. In (Jiang et al., 2013), they propose a quasi-static heuristic to optimize quality of service under security and schedulability constraints for dynamic task sets. In (Aminifar et al., 2017), the authors propose an optimal polynomial-time algorithm to distribute the available resources on a uniprocessor platform among applications at runtime, in order to maximize confidentiality under real-time constraints. Finally, in (Lesi et al., 2017), the authors consider the interplay between security overhead and quality of control, focusing only on uniprocessor task scheduling, without any networking and message scheduling/routing aspects.

In the context of the current state-of-the-art, the majority of previous studies are restricted to real-time systems and hard timing constraints. The resilience of network control systems against adversarial attacks, taking the stability constraints into account, remains an open problem.

\subsection{Preliminaries}

In this section, we describe some preliminary concepts used in this chapter.

\subsubsection{Security Level}

As discussed in Section 2.5 a block cipher can be used to preserve confidentiality and integrity of data. Here we discuss the trade-off between the timing overhead of a block cipher and the security level it provides.

Often block cipher algorithms have different key sizes and numbers of rounds in the encryption/decryption process. This property makes them suitable for 
many applications, since this makes it possible to exploit the trade-off between the security level required and the time overhead. For a specific block cipher algorithm, the rule of thumb is that the effort required to break it grows exponentially as larger key sizes or larger numbers of rounds are used. Therefore, larger key sizes and more rounds in running a block cipher will lead to a better security level. For a specific block cipher algorithm, one can presume a function that maps the key size and number of rounds used during encryption/decryption to the security level provided. This function grows exponentially with respect to the key size and the number of rounds for all block cipher algorithms and the exact relation depends on the type of cryptanalysis attack. The complexity of these cryptanalysis attacks are often reported as data complexity (number of plaintext-ciphertext pairs needed) (Borst et al., 1999; Contini et al., 1998; Knudsen and Meier, 2000) and/or computational complexity (Bogdanov et al., 2011; Tao and Wu, 2015). In order to have a more comprehensive definition of the security level for a specific block cipher, we consider the maximum of data and computational complexity, based on the best known attack against that block cipher. Therefore, we consider the following definition of security level:

Definition 5.2.1. Security level is expressed in bits and $n$-bit security means the attacker can break the cryptographic system using $2^{n}$ operations (Lenstra, 2004).

On the other hand, the timing overhead required for encryption and decryption increases also with the key size and the number of rounds. Therefore, the greater the amount of time allocated to running the encryption algorithm (bigger key size and/or more rounds), the higher the security level. As a result, different security levels can be considered depending on the overhead that the sender and receiver can tolerate. This trade-off is captured by a function called security trade-off function that can be derived for each specific block cipher.

Therefore, for a specific cryptographic algorithm security trade-off function is a function from the set of timing overheads to the set of security levels. Timing overheads are different computational overheads of the cryptographic algorithm and depend on the cryptographic parameters (key sizes and number 
of rounds) and the plaintext size. The set of security levels capture the $n$-bit security level provided by the encryption.

In the following we will consider two common block cipher algorithms and obtain the security trade-off function for them. From the five finalists of the AES competition (Nechvatal et al., 2001) we choose RC6 and Rijndael as two examples $^{1}$. The parameters we are interested in are the number of rounds and the key size these algorithms use for encryption/decryption. In order to evaluate the encryption/decryption overhead for these algorithms, we run several experiments on an embedded ARM processor. All of the following experiments to evaluate the timing overhead of Rijndael and RC6 are done on a Quad-core ARM Cortex-A57 processor with frequency fixed at 1.1 MHz.

In Rijndael (the winner of the AES competition), the designer can choose among five different key sizes (i.e., 128, 160, 192, 224, and 256 bits). Figure 5.1 shows that the encryption/decryption overhead increases linearly by increasing the key size. On the other hand, to find the security level for Rijndael for different key sizes, we look at the best known key recovery attacks (Bogdanov et al., 2011; Tao and Wu, 2015). The attacks use a method called a biclique attack and have a computational complexity slightly less than that of a brute-force attack. Figure 5.2 shows the $n$-bit security level for different key sizes, when using the biclique attack. As a result, the security trade-off function for Rijndael block cipher is obtained (see Table 5.4) based on Figure 5.1 and Figure 5.2.

In the RC6 algorithm, the key size can vary from 8 to 2040 bits and the number of rounds can vary from 1 to 255 rounds. The greater the key size or the number of rounds in encryption, the greater the security level that the algorithm provides, and the more time and resources that are needed to encrypt the message. Figure 5.3 shows how the encryption/decryption overhead increases as the key size and number of rounds in the RC6 algorithm are increased. According to this figure, increasing the number of rounds contributes more to the timing overhead than increasing the key size.

\footnotetext{
${ }^{1}$ The same approach can be applied for any other block ciphers e.g., Twofish, IDEA, Serpent, MARS, etc.
} 


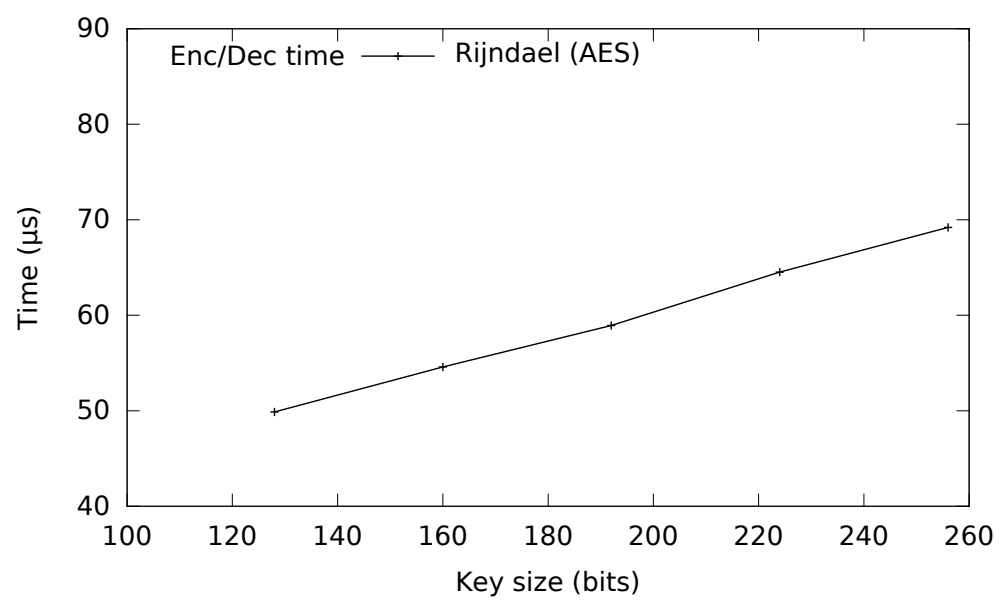

Figure 5.1: Encryption/Decryption time (in microseconds) for 16 bytes of plaintext versus key size for Rijndael.

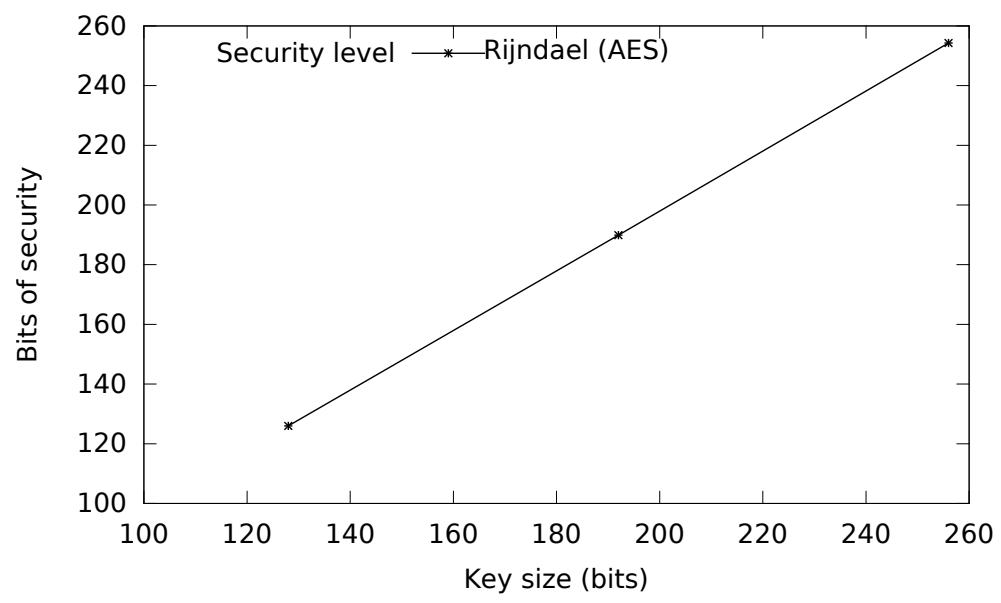

Figure 5.2: Security level versus key size for Rijndael. 


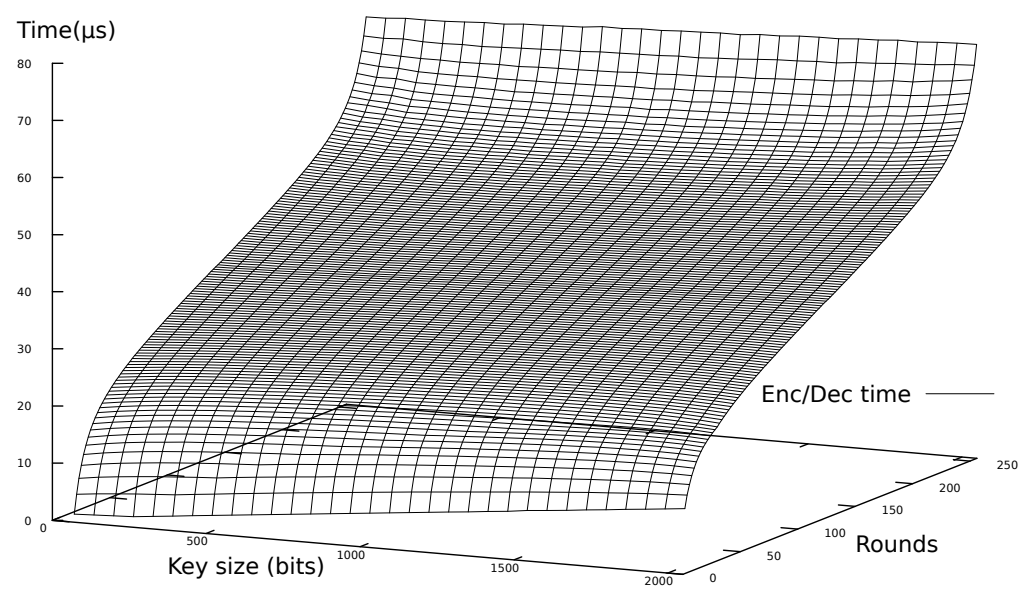

Figure 5.3: Encryption/Decryption time (in microseconds) for 16 bytes of plaintext versus number of rounds and key size for RC6 .

Table 5.1: Number of plaintext-ciphertext pairs required to mount a successful attack on RC6 (Borst et al., 1999).

\begin{tabular}{l|lllll} 
Rounds & $\mathrm{r}=8$ & $\mathrm{r}=12$ & $\mathrm{r}=16$ & $\mathrm{r}=20$ & $\mathrm{r}=24$ \\
\hline Linear attack to RC6 & $2^{62}$ & $2^{102}$ & $2^{152}$ & $2^{182}$ & $2^{222}$
\end{tabular}

On the other hand, increasing the key size only helps to increase the security level against a brute-force attack. If we assume that $b$ is the key size, then the attacker can find the key by testing $2^{b}$ different keys. Therefore, in the best case, the security level is b-bits. In order to show the security level of the RC6 algorithm for different rounds, we look at one of the most powerful attacks targeting this algorithm (Borst et al., 1999). The attack reports the data complexity (i.e., the number of plaintext-ciphertext pairs) required to successfully break the encryption. Table 5.1 shows the estimated number of plaintexts required to mount an attack on the RC6 algorithm for 8 to 24 rounds. Therefore, the security level of the RC6 algorithm with a key size of $b$ bits and $r$ rounds is the minimum of $b$ and the exponent related to $r$ in Table 5.1. Given that the result in Table 5.1 can be extended for more rounds, the security trade-off function for the RC6 block cipher can be obtained from this table and Figure 5.3. For example, if we consider the RC6 algorithm with a 512-bit key size, then according to Table 5.1 the security level for 12 rounds 
is 102 bits. Moreover, in Figure 5.3, the time to encrypt/decrypt 16 bytes of data using the RC6 algorithm with a 512-bit key and 12 rounds is $4.84 \mu \mathrm{s}$. Thus, the security trade-off function at the point $4.84 \mu \mathrm{s}$ is 102 bits. The rest of the pairs for the function can be obtained accordingly.

According to the two aforementioned block ciphers, the cryptography overhead increases with the security level. No matter what the block cipher algorithm and its parameters (e.g., key size and number of rounds) are, the relation between the cryptography overhead and the security level can be derived using the security trade-off function. In this chapter, we will use this function to provide a systematic methodology for maximizing the security level, while guaranteeing the stability of the control applications.

\subsubsection{Stability Analysis}

As we discussed in Section 2.3 in the context of networked systems, control applications may experience time-varying delays due to shared resources, which can lead to instability if not properly considered during design. The worstcase control performance of a system can be quantified as the upper bound on the gain from the uncertainty input (here, time-varying delays) to the plant output. In Section 2.3 we described the dependency between the stability of a control application and the latency and jitter experienced by its controller. The stability condition for control application $\Lambda_{i}$ is captured by Equation 2.5. Therefore, for control application $\Lambda_{i}$ we define the stability margin, denoted by $\delta_{i}$, as follows,

$$
\delta_{i}=\beta_{i}-\left(L_{i}+\alpha_{i} J_{i}\right)
$$

A non-negative $\delta_{i}$ guarantees the stability of the system.

\subsection{System Model}

In this chapter, we consider the system described in Section 2.1, which comprises a network of Ethernet switches that provides the infrastructure for connecting the sensors and controllers of $n$ control applications. 


\subsubsection{Network and Ethernet Switches}

We model the network and its topology as a graph $\boldsymbol{G}=(\boldsymbol{V}, \boldsymbol{E})$ where nodes $v_{i} \in \boldsymbol{V}$ are Ethernet switches, sensors, or controllers and the edges $\left[v_{i}-v_{j}\right] \in$ $\boldsymbol{E}$ are full-duplex physical links between these nodes. Figure 2.2 shows an example network topology with 14 nodes consisting of 8 Ethernet switches that connects 3 sensors to 3 controllers. We consider the model described in Section 2.1.2.

Ethernet switches in the network implement the IEEE 802.1Qbv-2015 TSN standard (IEEE Standard, 2015). According to this standard, each egress (output) port in a switch has up to 8 associated queues with strict-priority arbitration. An overview of an Ethernet TSN switch is depicted in Figure 2.3. As discussed in Section 2.1.2.1 all of the incoming messages to the switch go through the Forwarding Engine, where the output port is determined based on a look-up table that captures the routing. At the output port, the dispatcher assigns the message to the proper queue based on its priority. Finally, for queues with scheduled traffic, the switch opens the timed-gate at the end of the queue, based on the predefined schedule, to transfer the message. The output port and release time of a message is determined based on the forwarding and scheduling variables stored in the switch. Therefore, inside a switch we have two stored variables for each message.

- Output port for message $m_{i}^{j}$ arriving at switch $v_{k}$ is denoted by $\eta_{i k}^{j}$.

- Release time of message $m_{i}^{j}$ arriving at switch $v_{k}$ is denoted by $\gamma_{i k}^{j}$.

These variables are determined at design time using our stability-aware and security-aware algorithms, which will be elaborated in Section 5.7 and Section 5.8.

Note that the messages $m_{i}^{j}$ considered here are not the only ones traveling over the Ethernet network. Other messages of lower criticality levels (e.g., Rate-Constraint, and Best-Effort messages) are handled by the switches corresponding to their traffic classes. The time-triggered messages, which are the only ones of interest in this chapter, are handled at the highest priority level. Therefore, the maximum interference of the messages belonging to any of the other traffic classes is bounded by the transmission delay of the largest 
lower-criticality message. Due to newer standards (IEEE Standard, 2016), which allow preemption, and more advanced preemption models (Ashjaei et al., 2021) conforming to the standard, this worst-case interference delay is even smaller (Zhou et al., 2021b). For simplicity of discussion, in this thesis, we do not consider this delay but it is trivial to add it in the formulation.

\subsubsection{Delay and Jitter}

Once a message has been sampled in the sensor and is available for communication, there are different sources of delay on its way to the controller(s).

Crypto overhead is the delay due to encryption/decryption of the message in the sensor/controller to provide confidentiality, integrity, and authenticity. This cryptography delay is denoted as $c d^{2}$ and is determined by the parameters of the block cipher algorithm, as we have discussed in Section 5.2.1.

In addition to the crypto delay between sensing and actuation, a message experiences three types of delays in each switch along the path:

- Forwarding delay is the time from a message's arrival in a switch until the message is put in the outgoing queue ${ }^{3}$. Forwarding delay for a switch is denoted by $s d$.

- Scheduling delay is the time between placement of a message in the proper queue of its outgoing port until the time at which the switch starts sending the message. This delay depends on the release time of the message determined by the scheduling and routing algorithm at design time.

\footnotetext{
${ }^{2}$ Since block cipher algorithms usually have the same overhead for encryption and decryption we consider one variable representing both overheads. In cases where the overhead for encryption and decryption differs, two variables can be defined to trivially extend our approach.

${ }^{3}$ For the sake of simplicity, we consider that this delay is constant for all messages in all switches. If this is not the case the generalization is straight forward.
} 
- Transmission delay is the amount of time it takes to transmit the message on a link. The transmission delay is denoted by $l d_{i}$ and depends on the size of the message and the data-rate of the $\operatorname{link}^{4}$.

The end-to-end delay for a message going from a sensor to a controller is the sum of forwarding, scheduling, and transmission delays along all switches in the route. If the sensor and controller use end-to-end encryption then the crypto delay in the sensor and the controller also contributes to the end-toend delay. The end-to-end delay is different for different messages of the same flow (see Section 2.1.2 and Section 5.5) inside of a hyper-period. Therefore, for each control application, the controller may experience both delay and jitter.

\subsubsection{Control Applications}

We consider periodic control applications that are sensitive to jitter and latency described in Section 2.1.3.

In addition we assume that each control application has a certain relative security weight. The relative security weight of control application $\Lambda_{i}$ is captured as a number between zero and one and is denoted by $w_{i}$. The relative security weight depends on the type of control application and is provided by the designer. As we will show in Section 5.8, after guaranteeing a certain security level for each control application, available resources are used in order to further increase the level of security according to the relative security weight of the control application.

\subsubsection{Security Model}

To ensure the confidentiality and integrity of messages and provide authenticity for sensors, a block cipher algorithm (e.g. Rjindael, RC6, DES, Twofish, etc.) is used. The sensors use the block cipher algorithm to encrypt messages and create the MAC and the controllers use the decryption algorithm to decrypt messages and verify the MAC (see Section 2.5). The security level

\footnotetext{
${ }^{4}$ For the sake of simplicity, we consider that the data-rate of all links are identical and the transmission delay only depends on the message size. If this is not the case the generalization is straight forward.
} 
achieved for a certain message depends on several parameters (e.g., number of rounds and key size) of the underlying block cipher algorithm. In Section 5.2.1 we explained, using RC6 and Rjindael as two examples, how one can determine the security trade-off function between cryptography overhead and security level for a specific block cipher algorithm and its parameters. Thus, the security level $s l_{i}$ for all messages $m_{i}^{j}$ corresponding to control application $\Lambda_{i}$ is captured by,

$$
s l_{i}=f\left(c d_{i}\right),
$$

where $c d_{i}$ is the cryptography overhead and $f$ is the security trade-off function (see Section 5.2.1). The function depends on the block cipher algorithm and can be provided using the method described in Section 5.2.1. For a specific block cipher and a given cryptography overhead, the output of this function represents the security level (n-bit security as in Definition 5.2.1) provided by that block cipher corresponding to the cryptography overhead.

\subsection{Threat Model}

We assume that the attacker is able to compromise one (or a few) Ethernet switch(es) in the network. The goal of the attacker might be to:

- sniff the confidential messages from a sensor to a controller that pass through the compromised switch(es),

- modify the content of messages going through the compromised switch(es) so that the new message leads to destabilization of the control application, or

- impersonate the sensors and send arbitrary messages to the corresponding controllers.

As discussed in Section 2.5, a block cipher can be used to encrypt and decrypt messages between sensors and controllers to provide confidentiality, integrity, and authenticity in order to protect against the above goals. Therefore, to mitigate the attack, the security level provided by the block cipher algorithm should exceed the attacker's power. We assume the attacker's power is limited 
to $2^{N}$ operations. Hence, the attacker can break a block cipher that has less than $N$-bits security.

We assume here that the sensor and the controller nodes are secure and impossible for the attacker to compromise.

\subsection{Problem Formulation}

As we noted in Section 2.1.2, flow $f_{i}$ denotes a series of messages that originate from sensor $S_{i}$ and is sent to controller $C_{i}$. Message $m_{i}^{j}$ is the $j$-th message in this flow which is sent at the $j$-th sampling period from sensor $S_{i}$ to controller $C_{i}$. We assume all messages coming from the same flow are following the same route from the sensor to the controller.

Given $n$ control applications and a network of Ethernet switches as described in Section 5.3, we consider the following as input to our problem:

- the topology of the network, given by graph $\boldsymbol{G}=(\boldsymbol{V}, \boldsymbol{E})$;

- the switch forwarding delay, $s d$, and data rate $D$ for all links; and

- for each control application $\Lambda_{i}$ :

* the message size $\kappa_{i}$ (all the messages $m_{i}^{j}$ of the flow $f_{i}$ are assumed to have the same size $\kappa_{i}$ );

* the transmission delay $l d_{i}$ for messages $m_{i}^{j} \in f_{i}$ which is calculated as: $l d_{i}=\kappa_{i} / D$;

* controller $C_{i}$ with period $h_{i}$, which is also the period of messages received by $C_{i}$ from sensor $S_{i}$;

* model of the plant $P_{i}$;

* security importance $w_{i}$; and

* the block cipher algorithm being used, which provides the security trade-off function $f$ (see Equation 5.2).

We divide the problem formulation into two parts: (i) Stability-aware routing and scheduling, (ii) Security and stability-aware routing and scheduling. 
(i) In the first part we neglect the threat model assuming no security measures are needed. Hence, the goal is to schedule and route all messages in the network so that the control applications are guaranteed to be stable. Therefore, the outputs of our routing and scheduling algorithm are the release time (schedule) and output port (route) for each message in each Ethernet switch. These are captured by the values assigned to the following variables:

- output port $\eta_{i k}^{j}$; and

- release time $\gamma_{i k}^{j}$,

for each message $m_{i}^{j} \in \mathscr{M}$ arriving at switch $v_{k}$. These values are produced at design time and stored in the switches.

(ii) In the second part we consider the threat model. Therefore, the goal is to provide schedules and routes for all messages in the network in such a way that not only is the stability of control applications guaranteed, but that the system also achieves a certain security level. Therefore, the optimization goals are to 1) maximize the minimum security level over all applications, and 2) maximize the weighted sum of security levels for all control applications, beyond the minimum security level found at goal 1. Thus, the problem is to first find the maximum security level that all control applications can afford. Then, while maintaining this worst-case security level for all control applications, maximize the weighted sum of security levels, while also guaranteeing the stability of all control applications. The outputs of our routing and scheduling algorithm are:

a) crypto delay for each control application, captured by $c d_{i}$. This variable exposes the encryption/decryption parameters (key size and numbers of rounds) used by the block cipher algorithm in the sensor $S_{i}$ and the controller $C_{i}$.

b) the release time (schedule) and output port (route) for each message in each Ethernet switch. These are captured by the values assigned to the following variables:

- output port $\eta_{i k}^{j}$;

- release time $\gamma_{i k}^{j}$, 
for each message $m_{i}^{j} \in \mathscr{M}$ arriving at switch $v_{k}$.

These values $\left(c d_{i}, \eta_{i k}^{j}\right.$, and $\left.\gamma_{i k}^{j}\right)$ are determined at design time. $\eta_{i k}^{j}$, and $\gamma_{i k}^{j}$ are capturing the route and schedule and are stored in the switches. $c d_{i}$ represents the time overhead for encryption and decryption in the sensors and controllers and implicitly determines the encryption parameters (see Section 5.2.1).

In the next section we first discuss the necessity of jointly solving the routing and scheduling problem (the first part of the problem formulation). Then we demonstrate the need for integrating security measures in the route and schedule synthesis (the second part of the problem formulation). In Section 5.7 we address the first part of the problem formulation and demonstrate the effectiveness of the solution by way of experiments in Section 5.7.4. Finally, we address the second part of the problem formulation in Section 5.8 followed by experimental results.

\subsection{Motivational Example}

In this section, we shall demonstrate the security and stability aware Ethernet scheduling and routing problem using an example. We consider a system consisting of 3 control applications connecting through a network of 8 Ethernet switches, as shown in Figure 2.2. The parameters (period, $\alpha, \beta$ ) for all control applications are shown in Table 5.2. The control applications issue messages at the beginning of each period, as represented by the upright arrows in Figure 5.4. The hyper-period (the least common multiple of 10,20, and 30) is 60 and the control applications inject 11 messages into the network in each hyper-period.

We assume that all the messages have the same size and the transmission delay $(l d)$ for all messages in all links is equal to $1 \mathrm{~ms}$. The forwarding delay $(s d)$ for all switches is $0.05 \mathrm{~ms}$. If two messages are going out from the same port of a switch then one of them needs to be scheduled after the other one is delivered to the next switch, hence the time difference between these two consecutive messages is at least $1 \mathrm{~ms}$ (transmission delay). 
Table 5.2: Parameters of the example control applications.

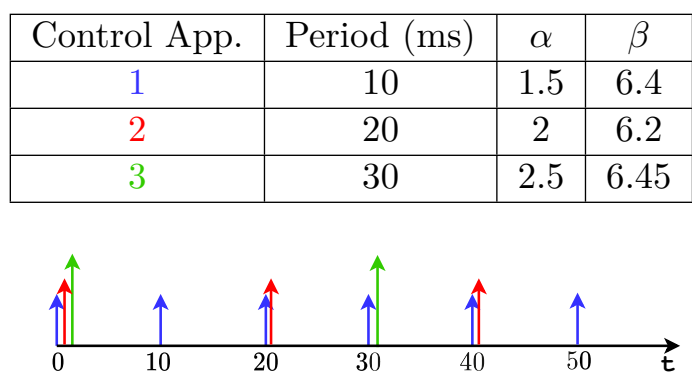

Figure 5.4: Issue time of messages from control applications.

Assuming that an attacker might compromise one or more Ethernet switches in the network and given that she is powerful enough to break up to 189-bit security, one must use a block cipher algorithm to encrypt/decrypt messages in the sensors/controllers. The parameters (e.g., key size and numbers of rounds) of the block cipher algorithm should be selected properly so that a security level greater than the attacker's power is provided.

Considering this configuration, one needs to provide routing and scheduling for these 11 messages that keeps the 3 control applications stable while taking the time for encryption and decryption into account. We first demonstrate the necessity of jointly solving the routing and scheduling problem in the absence of a security goal in Section 5.6.1. Then we motivate the need for integrating security measures in the route and schedule synthesis in Section 5.6.2. For readers' convenience we show a copy of Figure 2.2 (topology of the network) in Figure 5.5.

\subsubsection{Routing and Scheduling without Security Constraints}

In the absence of a security goal, the route and schedule synthesis problem can be solved using two different approaches: The first approach, based on fixed routing, uses a shortest path algorithm to determine routes, followed by a scheduling algorithm to determine start times for all message transmis- 


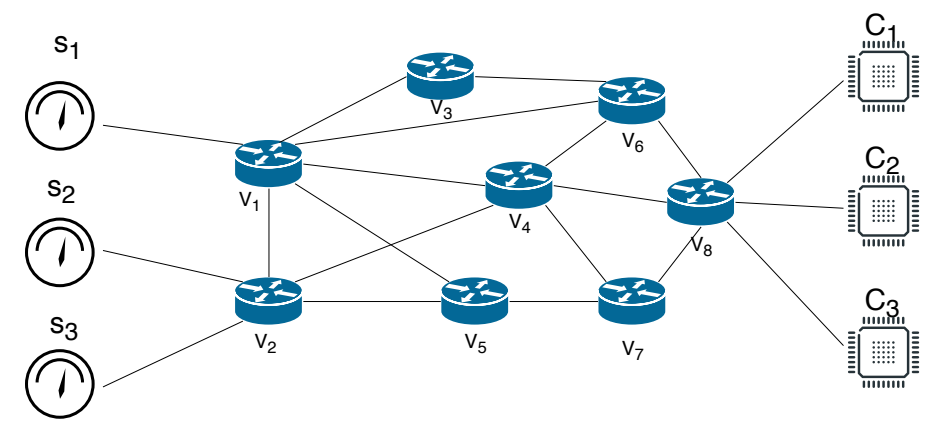

Figure 5.5: Network topology for motivational example.

sions across the network. The second approach, called integrated routing and scheduling, simultaneously determines the routes and schedules.

Fixed Routing (FR): Assuming shortest path, the routes for each control application are given in Equation 5.3 (the network topology is given in Figure 5.5). If we consider a fixed priority static scheduling and assuming that the control application $\Lambda_{2}$ has the highest priority and the control application $\Lambda_{3}$ has the lowest priority, the result of one possible schedule is provided in Table 5.3 under column FR.

This static schedule assumes that a message will be released right after being issued. The next switch then forwards the message $s d=0.05 \mathrm{~ms}$ (the forwarding delay) after receiving it, unless there already is another message in the queue. Here we explain how arrival times in Table 5.3 are determined for messages $m_{2}^{1}$ and $m_{1}^{1}$ as two examples. Message $m_{2}^{1}$ is released from $S_{2}$ at time 0. According to Equation 5.3 the route for this message is $S_{2} \rightarrow v_{2} \rightarrow v_{4} \rightarrow v_{8} \rightarrow C_{2}$. Therefore, $m_{2}^{1}$ arrives at $v_{2}$ at time $1(1 \mathrm{~ms}$ transmission delay in link). Then, since the message has the highest priority, switch $v_{2}$ forwards it at time 1.05 (0.05ms forwarding delay in switch) to $v_{4}$. Switch $v_{4}$ will receive the message at time 2.05 and send it at 2.1. Finally the message arrives at $C_{2}$ at time 4.15. Let us now consider message $m_{1}^{1}$. According to Equation 5.3 the route for this message is $S_{1} \rightarrow v_{1} \rightarrow v_{4} \rightarrow v_{8} \rightarrow C_{1}$. The message is released at 0 from $S_{1}$ and arrives at $v_{1}$ at 1 . It is released from $v_{1}$ at 1.05 . Then it arrives at $v_{4}$ at time 2.05. Since message $m_{2}^{1}$ has higher priority and is already scheduled to be released from the same port of $v_{4}$ at 
$\Lambda_{1}: S_{1} \rightarrow v_{1} \rightarrow v_{4} \rightarrow v_{8} \rightarrow C_{1}$

$\Lambda_{2}: S_{2} \rightarrow v_{2} \rightarrow v_{4} \rightarrow v_{8} \rightarrow C_{2}$

$\Lambda_{3}: S_{3} \rightarrow v_{2} \rightarrow v_{4} \rightarrow v_{8} \rightarrow C_{3}$.

Table 5.3: Arrival time of messages with FR and IRS approach.

\begin{tabular}{|c|c|c|c|}
\hline \multirow{2}{*}{} & \multirow{2}{*}{ issue } & \multicolumn{2}{|c|}{ arrival } \\
\cline { 3 - 4 } & & FR & IRS \\
\hline$m_{1}^{1}$ & 0 & 5.15 & 4.15 \\
\hline$m_{1}^{2}$ & 10 & 14.15 & 14.15 \\
\hline$m_{1}^{3}$ & 20 & 25.15 & 24.15 \\
\hline$m_{1}^{4}$ & 30 & 34.15 & 34.15 \\
\hline$m_{1}^{5}$ & 40 & 45.15 & 44.15 \\
\hline$m_{1}^{6}$ & 50 & 54.15 & 54.15 \\
\hline$m_{2}^{1}$ & 0 & 4.15 & 5.2 \\
\hline$m_{2}^{2}$ & 20 & 24.15 & 25.2 \\
\hline$m_{2}^{3}$ & 40 & 44.15 & 45.2 \\
\hline$m_{3}^{1}$ & 0 & 6.15 & 4.15 \\
\hline$m_{3}^{2}$ & 30 & 35.15 & 34.15 \\
\hline
\end{tabular}

2.1, message $m_{1}^{1}$ has to wait until $m_{2}^{1}$ is delivered to switch $v_{8}$ ( $1 \mathrm{~ms}$ transmission delay). Therefore, $m_{1}^{1}$ is released at 3.1 from switch $v_{4}$. In this case, message $m_{1}^{1}$ will arrive at $v_{8}$ at time 4.1 and will be released from $v_{8}$ at 4.15 , and finally delivered to $C_{1}$ at 5.15 . The remaining messages are scheduled accordingly and the arrival times are reported in Table 5.3 under column FR. The gray cells indicate messages that have been delayed because of contention in the network. Note that the end-to-end delay of a message is the difference between its arrival time and issue time. According to the arrival times in Table 5.3, when routes are pre-selected using the shortest path algorithm, the stability analysis of the control applications is as follows (see Section 5.2.2),

$$
\begin{aligned}
& \Lambda_{1}: L_{1}=4.15, R_{1}^{\mathrm{w}}=5.15, J_{1}=1 \Rightarrow \delta_{1}=0.75 \geqslant 0 \\
& \Lambda_{2}: L_{2}=4.15, R_{2}^{\mathrm{w}}=4.15, J_{2}=0 \Rightarrow \delta_{2}=2.05 \geqslant 0 \\
& \Lambda_{3}: L_{3}=5.15, R_{3}^{\mathrm{w}}=6.15, J_{3}=1 \Rightarrow \delta_{3}=-1.2<0 .
\end{aligned}
$$

Note that according to Equation 2.4, jitter for a control application is calculated as the difference between the worst-case and the best-case end-to-end delays of all messages related to that control application in a hyper-period. 
For example, for the first control application there are 6 messages in the hyperperiod. The worst-case end-to-end delay is $R_{1}^{\mathrm{w}}=5.15$ and the best-case endto-end delay is $R_{1}^{\mathrm{b}}=L_{1}=4.15$, which results in jitter $J_{1}=1$. Considering the stability analysis (Equation 5.4), the provided schedule cannot guarantee the stability of the third control application. It can be proven that for this example, with fixed routes as in Equation 5.3, it is impossible to provide any schedule that can guarantee the stability of all control applications, while an integrated routing and scheduling approach is able to synthesize such schedule. This demonstrates the need for an integrated routing and scheduling approach.

Integrated Routing and Scheduling (IRS): The second approach jointly provides routing and scheduling. This provides the flexibility to choose routes while the schedule is being built. In this case, one possible solution is as follows. The final routes are,

$$
\begin{aligned}
& \Lambda_{1}: S_{1} \rightarrow v_{1} \rightarrow v_{6} \rightarrow v_{8} \rightarrow C_{1} \\
& \Lambda_{2}: S_{2} \rightarrow v_{2} \rightarrow v_{5} \rightarrow v_{7} \rightarrow v_{8} \rightarrow C_{2} \\
& \Lambda_{3}: S_{3} \rightarrow v_{2} \rightarrow v_{4} \rightarrow v_{8} \rightarrow C_{3},
\end{aligned}
$$

and the arrival time of messages is is given in Table 5.3 under column IRS. According to this schedule and routing solution, the stability analysis of the control applications is as follows,

$$
\begin{aligned}
& \Lambda_{1}: L_{1}=4.15, J_{1}=0 \Rightarrow \delta_{1}=2.25 \geqslant 0 \\
& \Lambda_{2}: L_{2}=5.2, J_{2}=0 \Rightarrow \delta_{2}=1 \geqslant 0 \\
& \Lambda_{3}: L_{3}=4.15, J_{3}=0 \Rightarrow \delta_{3}=2.3 \geqslant 0 .
\end{aligned}
$$

Accordingly, the new integrated routing and scheduling strategy guarantees the stability of all control applications. Note that in the provided route and schedule no contention happens in the network, resulting in no jitter for the control applications. Moreover, the flexibility in selecting routes while generating the schedule makes it possible to synthesize a route and schedule that can guarantee the stability of all control applications. This method is what we describe in Section 5.7 as stability-aware method to solve part (i) of the problem formulated in Section 5.5. In the next section, we continue the same example to motivate the need for integrating security measures in the route and schedule synthesis. 
Table 5.4: Security trade-off function for Rijndael. The overhead for encryption/decryption is based on experiments on a Quad-core ARM Cortex-A57 processor with frequency fixed at $1.1 \mathrm{MHz}$.

\begin{tabular}{|c|c|c|}
\hline \multicolumn{2}{|c|}{ Overhead $(\mu s)$} & \multirow{2}{*}{ security level (bits) } \\
\hline 16 bytes & 160 bytes & \\
\hline \hline 49.8 & 498 & 126 \\
\hline 54.5 & 545 & 158 \\
\hline 58.9 & 589 & 189.9 \\
\hline 64.5 & 645 & 222.1 \\
\hline 69.2 & 692 & 254.3 \\
\hline
\end{tabular}

\subsubsection{Routing and Scheduling with Security Constraints}

There are two approaches to implementing the security requirements while synthesizing routes and schedules. The first (naive) approach is to begin by reserving a time interval in sensors and controllers for encryption and decryption and then synthesizing routes and schedules. The other approach is to integrate security measures in the routing and scheduling synthesis and consider the time required for encryption and decryption while synthesizing the routes and schedules.

In this example, we assume that the encryption algorithm is Rijndael and that the sensors and controllers are using the same processor as the one used in Section 5.2.1. The security trade-off function for Rijndael obtained from Figure 5.1 and Figure 5.2 is shown in Table 5.4. Note that the overhead values reported in the figures are for 16 bytes of data. In this example, the messages are considered to be 160 bytes and therefore the overhead for a 160 bytes message is also reported in Table 5.4.

First we consider the naive approach and assume the designer reserves $0.5 \mathrm{~ms}$ for encryption and decryption. Therefore, a message is released for transmission $0.5 \mathrm{~ms}$ after being issued by the sender and is available at the controller $0.5 \mathrm{~ms}$ after arrival. If we use the IRS method describe in the previous section the routes and arrival times are reported in Equation 5.8 and Table 5.5, respectively. Considering this route and schedule synthesis, the sender can 
Table 5.5: Arrival time of messages using the naive approach for integrating security.

$\Lambda_{1}: S_{1} \rightarrow v_{1} \rightarrow v_{6} \rightarrow v_{8} \rightarrow C_{1}$

$\Lambda_{2}: S_{2} \rightarrow v_{2} \rightarrow v_{5} \rightarrow v_{7} \rightarrow v_{8} \rightarrow C_{2}$

$\Lambda_{3}: S_{3} \rightarrow v_{2} \rightarrow v_{4} \rightarrow v_{8} \rightarrow C_{3}$,

\begin{tabular}{|c|c|c|c|}
\hline & \multirow{2}{*}{ issue } & \multirow{2}{*}{ release } & arrival \\
\cline { 4 - 4 } & & & IRS \\
\hline$m_{1}^{1}$ & 0 & 0.5 & 4.65 \\
\hline$m_{1}^{2}$ & 10 & 10.5 & 14.65 \\
\hline$m_{1}^{3}$ & 20 & 20.5 & 24.65 \\
\hline$m_{1}^{4}$ & 30 & 30.5 & 34.65 \\
\hline$m_{1}^{5}$ & 40 & 40.5 & 44.65 \\
\hline$m_{1}^{6}$ & 50 & 50.5 & 54.65 \\
\hline$m_{2}^{1}$ & 0 & 0.5 & 5.7 \\
\hline$m_{2}^{2}$ & 20 & 20.5 & 25.7 \\
\hline$m_{2}^{3}$ & 40 & 40.5 & 45.7 \\
\hline$m_{3}^{1}$ & 0 & 0.5 & 4.65 \\
\hline$m_{3}^{2}$ & 30 & 30.5 & 34.65 \\
\hline
\end{tabular}

spend $0.5 \mathrm{~ms}$ on encryption (from issuing the message to releasing it for communication on the network). According to Table 5.4, this means that the maximum possible security level is 126 bits, which is provided using Rijndael with a 128-bit key size. Moreover, the same $0.5 m s$ overhead must be considered for decryption on the controller side; hence, the end-to-end delay of each message will increase by $0.5 \mathrm{~ms}$. Therefore, by adding $0.5 \mathrm{~ms}$ for encryption and decryption, the system has 126 bits of security and all of the control applications are kept stable (see Equation 5.7).

$$
\begin{aligned}
& \Lambda_{1}: L_{1}=4.65+0.5, J_{1}=0 \Rightarrow \delta_{1}=1.25 \geqslant 0 \\
& \Lambda_{2}: L_{2}=5.7+0.5, J_{2}=0 \Rightarrow \delta_{2}=0.0 \geqslant 0 \\
& \Lambda_{3}: L_{3}=4.65+0.5, J_{3}=0 \Rightarrow \delta_{3}=1.3 \geqslant 0 .
\end{aligned}
$$

Note that the stability constraint for control application $\Lambda_{2}$ is zero, hence there is no more room for increasing the encryption/decryption overhead beyond $0.5 \mathrm{~ms}$. This example shows that even if the designer could shift all of the scheduled times in all switches to allocate time for encryption in the sensors, 
it is still impossible to provide more than $0.5 \mathrm{~ms}$ (126 bits security), which is well below the capabilities of the attacker, which is 189 bits for this example. Therefore, it is important to integrate the security optimization goals during the synthesis of the routes and schedules.

In the security and stability-aware synthesis approach, the security goals are integrated with the routing and scheduling constraints and the routes and schedules are provided such that the security objectives are satisfied. For example, if we want to maximize the minimum security level achievable for all messages, then Table 5.6 and Equation 5.9 show the new schedule and route. Using this new route and schedule, the stability analysis of the three control applications is provided in Equation 5.10. Note that the messages are ready for use by the controller only after they are decrypted, hence $0.6 \mathrm{~ms}$ adds up to the end-to-end delay of all messages for decryption overhead. Based on these results, the sensors/controllers can spend $0.6 \mathrm{~ms}$ on encryption/decryption which, according to Table 5.4, provides a 189.9 bit security level for all control applications. This security level is above the assumed capabilities of the attacker. Thus, this example shows that an integrated approach allows us to find routes and schedules that keep all control applications stable while reserving more time for encryption/decryption, thereby enabling us to improve the security level of the system beyond the capabilities of an attacker.

$$
\begin{aligned}
& \Lambda_{1}: L_{1}=4.75+0.6=5.35, J_{1}=0 \Rightarrow \delta_{1}=1.05 \geqslant 0 \\
& \Lambda_{2}: L_{2}=4.75+0.6=5.35, J_{2}=0 \Rightarrow \delta_{2}=0.85 \geqslant 0 \\
& \Lambda_{3}: L_{3}=5.8+0.6=6.4, J_{3}=0 \Rightarrow \delta_{3}=0.05 \geqslant 0
\end{aligned}
$$

With the schedule provided in Table 5.6, all of the control applications are guaranteed to be stable, and they are also secure assuming that the attacker's power is bounded to $2^{189.9}$ operations, which is above the power of the attacker being considered in this example. This schedule provides the maximum security level achievable for all applications. One can think of better security levels for some of the applications. In this example, 189.9 is the maximum security level that can be provided for application $\Lambda_{3}$, but the two other control applications can have better security levels (enabled by different routes and schedules). Although the attacker is assumed to have limited power in 
$\Lambda_{1}: S_{1} \rightarrow v_{1} \rightarrow v_{6} \rightarrow v_{8} \rightarrow C_{1}$

$\Lambda_{2}: S_{2} \rightarrow v_{2} \rightarrow v_{4} \rightarrow v_{8} \rightarrow C_{2}$

$\Lambda_{3}: S_{3} \rightarrow v_{2} \rightarrow v_{5} \rightarrow v_{7} \rightarrow v_{8} \rightarrow C_{3}$

Table 5.6: Arrival time of messages for maximizing the minimum security level (192 bits).

\begin{tabular}{|c|c|c|c|}
\hline & \multirow{2}{*}{ issue } & \multirow{2}{*}{ release } & arrival \\
\cline { 4 - 4 } & & & IRS \\
\hline$m_{1}^{1}$ & 0 & 0.6 & 4.75 \\
\hline$m_{1}^{2}$ & 10 & 10.6 & 14.75 \\
\hline$m_{1}^{3}$ & 20 & 20.6 & 24.75 \\
\hline$m_{1}^{4}$ & 30 & 30.6 & 34.75 \\
\hline$m_{1}^{5}$ & 40 & 40.6 & 44.75 \\
\hline$m_{1}^{6}$ & 50 & 50.6 & 54.75 \\
\hline$m_{2}^{1}$ & 0 & 0.6 & 4.75 \\
\hline$m_{2}^{2}$ & 20 & 20.6 & 24.75 \\
\hline$m_{2}^{3}$ & 40 & 40.6 & 44.75 \\
\hline$m_{3}^{1}$ & 0 & 0.6 & 5.8 \\
\hline$m_{3}^{2}$ & 30 & 30.6 & 35.8 \\
\hline
\end{tabular}

this example, it is plausible that the attacker's capabilities could improve throughout the time during which the system is in use. Therefore, while it is important to find routes and schedules that meet a certain minimum security level (given by the assumed capabilities of an attacker), we additionally consider an optimization problem to maximize the security level of the system.

In the following we will demonstrate a second optimization problem where, assuming that the minimum security level is calculated over all control applications, the goal is to further maximize the weighted sum of security levels. Thus, we try to maintain the best security level achievable for all control applications and then distribute the remaining time slack with regard to the security importance (weights) of the control applications. In the above example, if we consider the goal of maximizing the weighted sum of security levels and assuming that the weights $\left(\omega_{i}\right)$ are equal to 1 for all control applications, then Table 5.7 shows the new schedule (the routes remain the same as in Equation 5.9 and the stability analysis of the three control applications 
Table 5.7: Arrival time of messages for maximizing the weighted sum of security levels.

\begin{tabular}{|c|c|c|c|}
\hline & \multirow{2}{*}{ issue } & \multirow{2}{*}{ release } & arrival \\
\cline { 4 - 4 } & & & IRS \\
\hline$m_{1}^{1}$ & 0 & 0.7 & 4.85 \\
\hline$m_{1}^{2}$ & 10 & 10.7 & 14.85 \\
\hline$m_{1}^{3}$ & 20 & 20.7 & 24.85 \\
\hline$m_{1}^{4}$ & 30 & 30.7 & 34.85 \\
\hline$m_{1}^{5}$ & 40 & 40.7 & 44.85 \\
\hline$m_{1}^{6}$ & 50 & 50.7 & 54.85 \\
\hline$m_{2}^{1}$ & 0 & 0.7 & 4.85 \\
\hline$m_{2}^{2}$ & 20 & 20.7 & 24.85 \\
\hline$m_{2}^{3}$ & 40 & 40.7 & 44.85 \\
\hline$m_{3}^{1}$ & 0 & 0.6 & 5.8 \\
\hline$m_{3}^{2}$ & 30 & 30.6 & 35.8 \\
\hline
\end{tabular}

is provided in Equation 5.11).

$$
\begin{aligned}
& \Lambda_{1}: L_{1}=4.85+0.7=5.55, J_{1}=0 \Rightarrow \delta_{1}=0.85 \geqslant 0 \\
& \Lambda_{2}: L_{2}=4.85+0.7=5.55, J_{2}=0 \Rightarrow \delta_{2}=0.65 \geqslant 0 \\
& \Lambda_{3}: L_{3}=5.8+0.6=6.4, J_{3}=0 \Rightarrow \delta_{3}=0.05 \geqslant 0
\end{aligned}
$$

According to the schedule, applications $\Lambda_{1}$ and $\Lambda_{2}$ have $0.7 \mathrm{~ms}$ to spend for encryption/decryption, hence they provide 254.3 bits of security.

In conclusion, we have demonstrated the need to maximize the minimum security level across all control applications in the system - all in the context of finding Ethernet schedules and routes that meet control stability constraints. Such optimization maximizes the capability that an attacker needs to possess to compromise the system. We have also demonstrated that there is an opportunity to further improve the security level of some control applications, in order to address growing attacker capabilities throughout the deployment of the system. This method is what we describe as security and stabilityaware method in Section 5.8 to solve part (ii) of the problem formulation in Section 5.5. 


\subsection{Stability-Aware Routing and Scheduling}

This section presents our approach for routing and scheduling of control messages with stability constraints, part (i) of the problem formulation in Section 5.5. In particular, we present a stability-aware SMT formulation and efficient heuristics to find port assignments and release times for all messages and network switches so that all control applications are guaranteed to be stable. Satisfiability Modulo Theory (SMT) is a decision problem that is designed to determine satisfiability of first-order logic with respect to some background theories such as integer arithmetic or bit vectors (Biere et al., 2009). If the constraints are satisfiable, SMT solvers provide a solution to the given variables. In the following the constraints to be formulated using SMT are described.

\subsubsection{Constraints With Respect to Routing and Scheduling}

- Topology constraint: The selected output node should be among the nodes that are connected to the current node.

$$
\begin{aligned}
& \forall i, j\left|m_{i}^{j} \in \mathscr{M}, \forall k\right| v_{k} \in \boldsymbol{V}: \\
& \text { if } \eta_{i k}^{j}=v_{l} \text { then }\left[v_{k}-v_{l}\right] \in \boldsymbol{E} .
\end{aligned}
$$

- Contention-free constraint: If two messages are waiting in the queues of the same port of a switch, then one of them should wait until the other one is released into the link. We consider this waiting time to be equal to the transmission delay $(l d)$ of that message.

$$
\begin{aligned}
& \forall i, j, r, s\left|m_{i}^{j}, m_{r}^{s} \in \mathscr{M}, \forall k\right| v_{k} \in \boldsymbol{V}: \\
& \text { if } \eta_{i k}^{j}=\eta_{r k}^{s} \quad \text { and } \quad(i \neq r \quad \text { or } \quad j \neq s) \\
& \text { then }\left|\gamma_{i k}^{j}-\gamma_{r k}^{s}\right| \geqslant l d .
\end{aligned}
$$

- Transposition constraint: The release time of a message should be after the release time of the same message from the predecessor node plus the link transmission delay $(l d)$ plus the switch forwarding delay 
$(s d)$

$$
\begin{aligned}
& \forall i, j\left|m_{i}^{j} \in \mathscr{M}, \forall k, l\right| v_{k}, v_{l} \in \boldsymbol{V}: \\
& \text { if } \eta_{i k}^{j}=v_{l} \text { then } \gamma_{i l}^{j} \geqslant \gamma_{i k}^{j}+s d+l d .
\end{aligned}
$$

- No-loop constraint: For each message there should not be any two switches that forward the message to the same destination.

$$
\begin{aligned}
& \forall i, j\left|m_{i}^{j} \in \mathscr{M}, \forall k\right| v_{k} \in \boldsymbol{V}, \forall l \mid v_{l} \in \boldsymbol{V}: \\
& \text { if } k \neq l \quad \text { then } \eta_{i k}^{j} \neq \eta_{i l}^{j} .
\end{aligned}
$$

- Route constraint: For each message there should be a route between the source (sensor) and destination (controller) of the control application to which message relates. $\boldsymbol{R}_{i}$ is the set of all possible routes from $S_{i}$ to $C_{i}$.

$$
\begin{aligned}
& \forall i, j \mid m_{i}^{j} \in \mathscr{M}, \exists\left\{S_{i}, v_{l_{1}}, v_{l_{2}}, \ldots, v_{l_{p}}, C_{i}\right\} \in \boldsymbol{R}_{i}: \\
& \quad\left(\eta_{i S_{i}}^{j}=v_{l_{1}}\right) \wedge\left(\eta_{i l_{1}}^{j}=v_{l_{2}}\right) \wedge \cdots \wedge\left(\eta_{i l_{p}}^{j}=C_{i}\right) .
\end{aligned}
$$

\subsubsection{Stability Constraints}

The two parameters impacting stability of control applications are latency and jitter, as discussed in Section 2.3. In order to compute these two parameters, we consider the end-to-end delay of message $m_{i}^{j}$ from sensor $S_{i}$ to controller $C_{i}$, denoted by $e 2 e_{i}^{j}$. This end-to-end delay might vary, inside a hyper-period, for different messages in the flow from $S_{i}$ to $C_{i}$. The end-to-end delay $e 2 e_{i}^{j}$ is equal to the release time of that message from the last switch in the route, plus the $l d$ of the link between this switch and the controller minus the initial release time from the sensor. If we consider the best-case delay for the controller $C_{i}$ as $\min _{j}\left\{e 2 e_{i}^{j}\right\}$ and the worst-case delay as $\max _{j}\left\{e 2 e_{i}^{j}\right\}$, then the latency $L_{i}$ and jitter $J_{i}$ for control application $\Lambda_{i}$ is computed as follows,

$$
L_{i}=\min _{j}\left\{e 2 e_{i}^{j}\right\}, \quad J_{i}=\max _{j}\left\{e 2 e_{i}^{j}\right\}-\min _{j}\left\{e 2 e_{i}^{j}\right\} .
$$

Given the model of the plant $P_{i}$ and controller $C_{i}$, we use the Jitter Margin toolbox to generate the linear lower bound for stability of the plant and compute the coefficients $\alpha_{i}$ and $\beta_{i}$ in Equation 5.1 (see Section 2.3). 
- Stability constraint: All control applications should be stable. Hence, The stability margin defined by Equation 5.1 should hold for all control applications.

$$
\delta_{i} \geqslant 0, \quad \forall \Lambda_{i}
$$

We use SMT to solve the problem captured by the above constraints. We formulate the constraints using the SMT-LIB (Barrett et al., 2010) library, then we use a solver to check their satisfiability. If the conjunction of these constraints is satisfiable, the solver associates a value to all $\eta_{i k}^{j}$ and $\gamma_{i k}^{j}$ for each message $m_{i}^{j}$ at each node $v_{k}$ of the network, thus providing the solution to the formulated problem.

\subsubsection{Improving scalability}

Routing and static scheduling for time-triggered messages are known to be NP-complete problems (Craciunas et al., 2016; Steiner, 2010), hence the scalability issue. Considering this inherent complexity of the network communication synthesis problem, we propose techniques to make a trade-off between the quality of synthesized solutions and the complexity of the synthesis process. Concretely, we propose two heuristics to address this scalability issue and to improve synthesis efficiency without major loss of quality.

\subsubsection{Route Subset}

This heuristic is proposed to reduce the routing complexity. In our basic solution, we consider all possible routes for each flow when searching for a schedulable solution. Here, we use the designers' knowledge of the network and control applications to reduce the size of the problem by eliminating the routes that are unlikely to be used. For example, the designer might consider only the first $K$ shortest routes for each control application. In order to apply this approach to the SMT formulation, we only need to consider the route subsets provided by the designer when formulating the route constraint (Equation 5.16). 


\subsubsection{Incremental Synthesis}

For the second method we divide the hyper-period into several time slices. Thus, the algorithm solves several smaller problems in an incremental fashion. In the first stage, the messages instantiated inside the first time slice are scheduled/routed solving the SMT formulation for the decision variables corresponding to this subset of messages. In the subsequent stages, schedules and routes are synthesized by running the SMT solver for the decision variables corresponding to the messages that are instantiated in the corresponding time slice and considering the decision variables that were determined in the previous stages to be fixed. The incremental algorithm terminates after all message instances have been considered. The above incremental technique reduces the number of messages that must be scheduled in each stage of the algorithm, resulting in reduced synthesis time.

Note that by applying these heuristics the solver searches within a subset of all possible solutions and, therefore, in some cases it might end up without a solution, even if such a solution exists and could be reached solving the initial, complete SMT formulation (See experimental results in Section 5.7.4.).

\subsubsection{Experimental Setup and Results}

In this section, we experimentally evaluate the efficiency of our proposed stability-aware routing and scheduling technique. We perform three experiments followed by a real-life example. Our experiments are carried out using Z3 (De Moura and Bjørner, 2008), a state-of-the-art SMT solver. They were run on a regular desktop computer with a $2.67 \mathrm{GHz}$ Xeon $\mathrm{CPU}$ and $6 \mathrm{~GB}$ of RAM. For the three experiments, we randomly choose control applications from a database with inverted pendulums, ball and beam processes, DC servos, and harmonic oscillators. These plants are considered to be representative for realistic control applications and are extensively used for experimental evaluation in the literature (Åström and Wittenmark, 1997). The first two experiments show how our approaches scale in terms of synthesis time. In both experiments, we run 60 different synthesis problems on a network of 35 nodes (10 sensors, 10 controllers, and 15 Ethernet switches). For each synthesis problem, these 10 control applications are chosen randomly, as discussed previously. To show the impact of our incremental synthesis heuristic, we run 


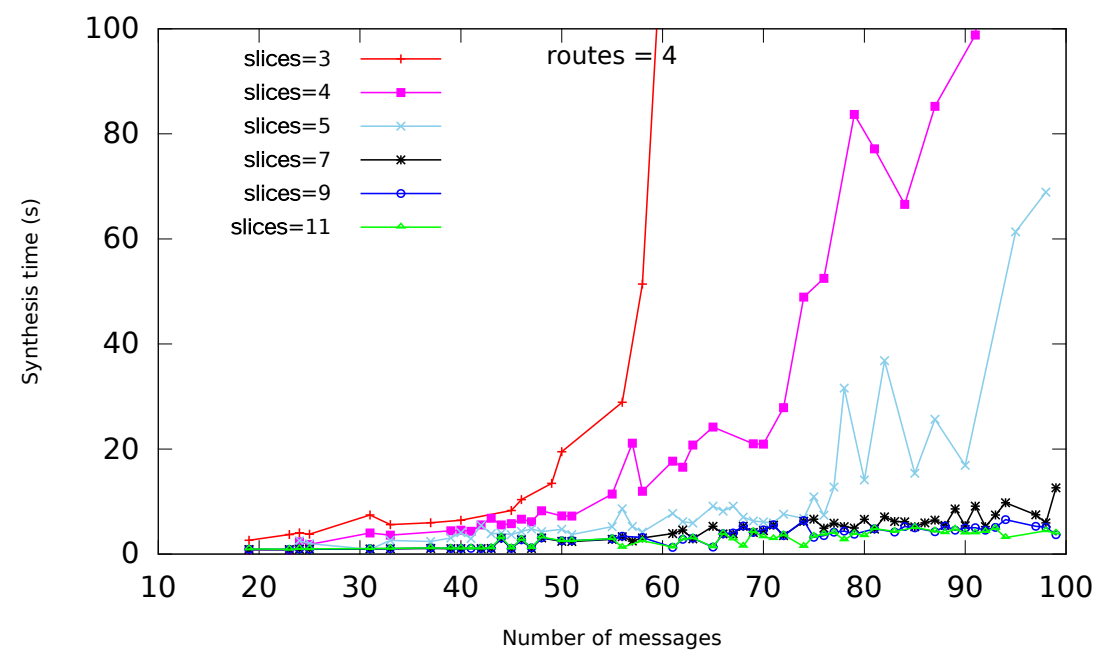

Figure 5.6: Scalability of the incremental synthesis heuristic.

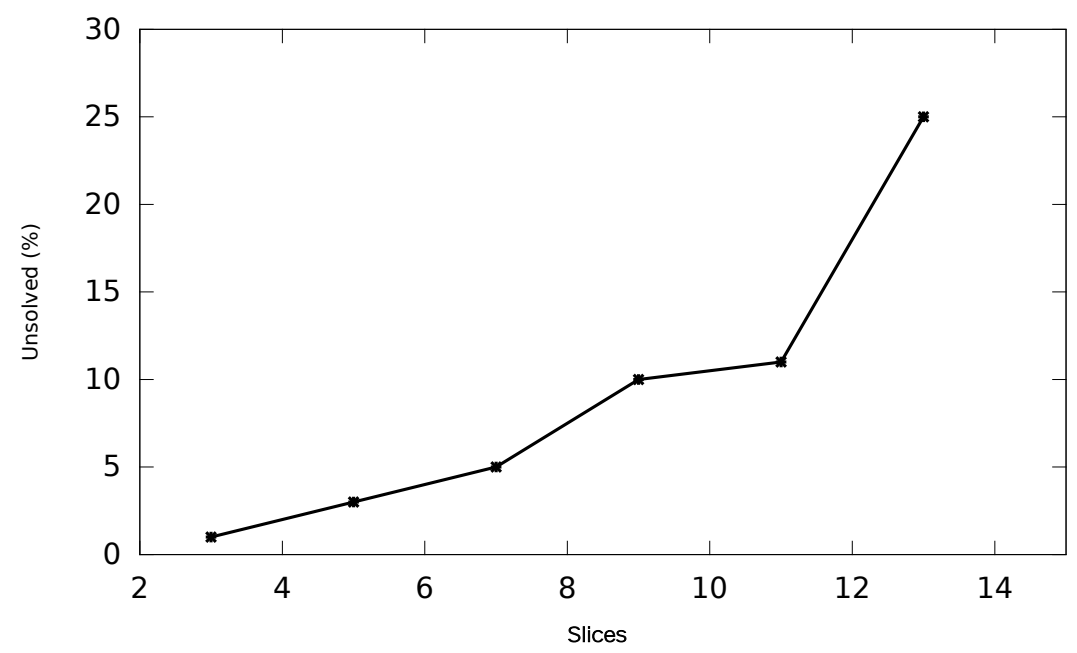

Figure 5.7: Percentage of unsatisfied problems with incremental synthesis. 


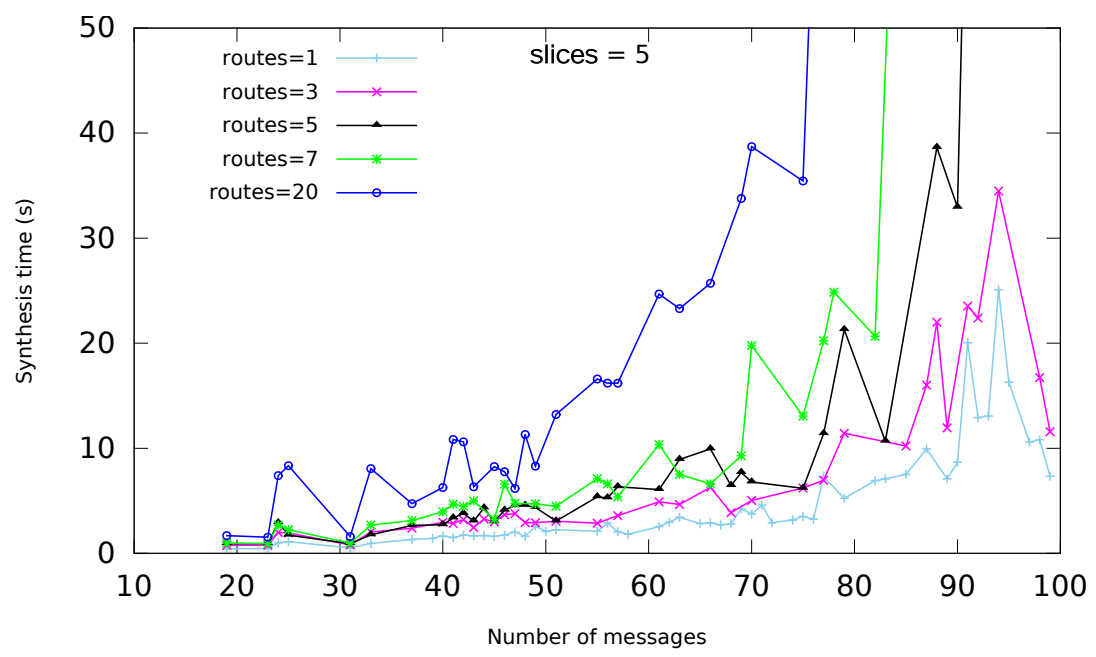

Figure 5.8: Scalability of the route subset heuristic.

hese 60 different problems 6 times, each time changing the number of time slices considered in the synthesis process. The result is shown in Figure 5.6. In this figure, each point represents the synthesis time of a particular problem. From the graph we can see that increasing the number of time slices dramatically reduces synthesis time. In our experiments, without the incremental approach (i.e., slices $=1$ ), the solver did not produce any results after a whole day for the problems with more than 80 messages, while the same problems are solved in less than a minute when we increase the number of slices to 5 . Note that all problems have been run considering 4 alternative routes for each message.

As discussed previously in this section, with the incremental approach we pay for the speed-up by only partially exploring the solution space. Figure 5.7 shows the percentage of cases in which a solution (all controllers are guaranteed to be stable) has not been found, depending on the number of time slices employed. Observe that, while execution times are already dramatically reduced for five slices compared to three (see Figure 5.6), the quality of exploration with five or seven slices is still very good. 


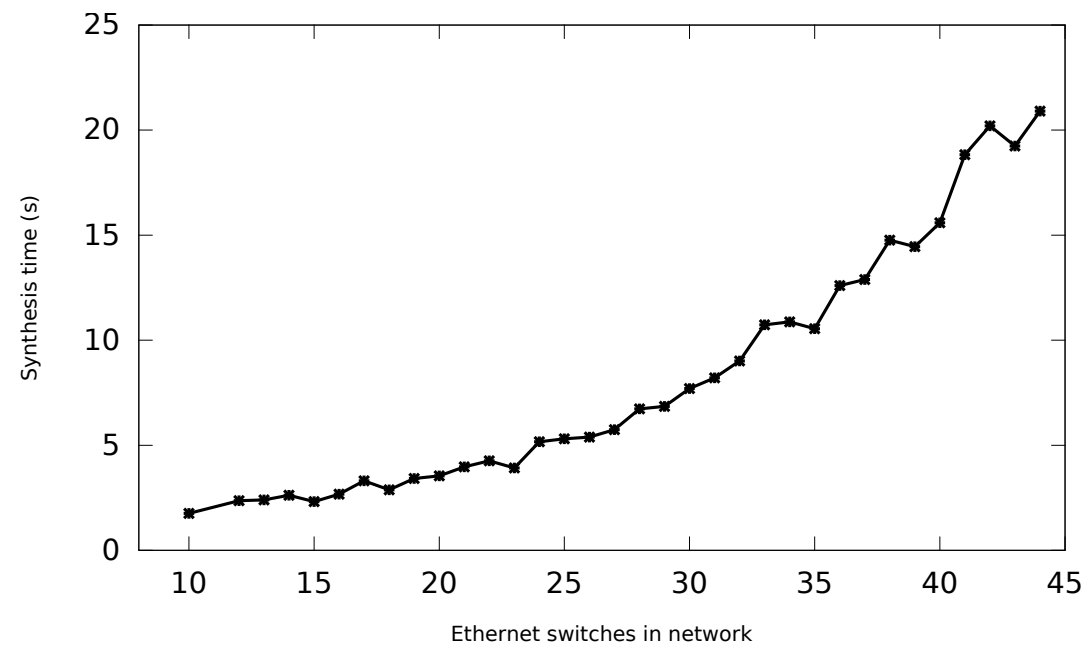

Figure 5.9: Scalability of the proposed approach for big networks.

In order to show the impact of providing a subset of alternative routes, we run the same 60 problems 5 times, each time changing the number of alternative routes while keeping the number of time slices to 5 . The results of this experiment are shown in Figure 5.8. The trend in the graph shows that decreasing the number of possible routes between sensors and controllers in the network reduces the synthesis time. On the other hand, our experiments show that reducing the set of alternative routes per application to 1 or 2 will result in an unsolved problem rate of more than $90 \%$, while for 3 and more sets of routes less than $10 \%$ remained unsolved.

The third experiment shows how synthesis time scales with the number of nodes in the network. In this experiment, we choose 10 control applications that generate 45 messages in one hyper-period. Then for each experiment we change the number of Ethernet switches in the underlying network. Given the number of switches in the network, we randomly generate the topology based on the Erdős-Rényi graph model (Erdős, Rényi, et al., 1960) and randomly connect the 10 sensors and 10 controllers to this topology. For this experiment we use the route subset heuristic with 4 route alternatives and the incremental synthesis heuristic with 4 slices. Figure 5.9 shows the synthesis time for different numbers of switches in the network. 
Finally, we have also applied our approach to a real-life example from General Motors. The example consists of 20 sensors (camera, radar, and lidar) and electronic control units for surround view perception, object tracking, active safety functions, and autonomous vehicle control. These 20 control applications are communicating through a network of 8 Ethernet switches, which are connected based on the topology depicted in Figure 2.2. For each of these 20 control applications the constant coefficients in stability curve, $\alpha$ and $\beta$ in Equation 5.1, are calculated as discussed in Section 2.3. The parameters (period, $\alpha$, and $\beta$ ) for five of these control applications are shown in Table 5.8.

The packet size for all of these control applications is 1500 bytes and the Ethernet switches and links are assumed to be $10 \mathrm{Mbit} / \mathrm{s}$. Therefore, the transmission delay for each link and each message is $l d=1.2 \mathrm{~ms}$. The forwarding delay for all switches is considered to be $s d=5 \mu s$. 


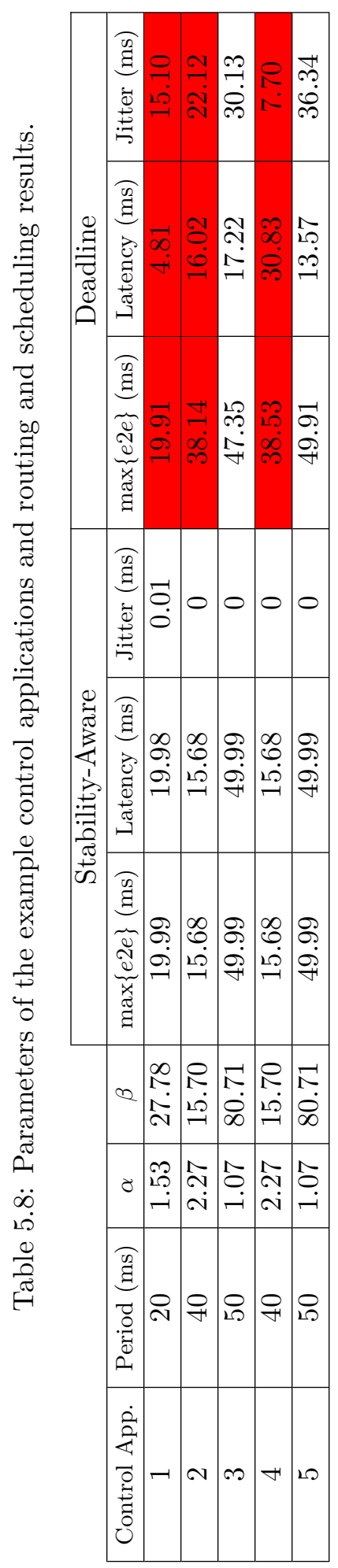


The synthesis problem for our set of 20 applications comprises the scheduling and routing of 106 messages (generated in the hyper-period of length $200 \mathrm{~ms}$ ). We have applied our synthesis approach providing 3 alternative routes for each message and considering 5 time slices. The synthesis time was 112 seconds and all 20 applications satisfied the worst-case stability constraints. We show the resulting maximal end-to-end delay, latency, and jitter for five out of 20 control applications in Table 5.8 under the Stability-Aware column. In order to highlight the importance of control-aware communication synthesis with explicit consideration of the stability conditions, we run an additional experiment with the identical setting as before. However, instead of imposing the stability constraints (Equation 5.18 and Equation 5.1), we impose a constraint on the worst-case delay of each message to not exceed its period. This approach represents the state of the art (i.e., scheduling and routing with implicit hard deadlines). The results are shown in Table 5.8 under the Deadline column. Analyzing the latencies and jitters, out of the five applications, three (highlighted in the table) turn out to be potentially unstable in the worst case. From the whole set of 20 applications, only 14 satisfied the worst-case stability conditions, further demonstrating the importance of our stability-aware routing and scheduling approach.

\subsection{Security and Stability-Aware Routing and Scheduling}

This section presents our approach, which is called security and stability-aware routing and scheduling, to address part (ii) of the problem formulation in Section 5.5. Similar to the stability-aware solution proposed in Section 5.7, we present an SMT formulation and efficient heuristics to find crypto delay, port assignment, and release time values for all messages and network switches. On top of the stability guarantee for the control applications in this section our goal is also to maximize the security level for the control applications. In the following additional constraints to the ones stated in Section 5.7 are described. 


\subsubsection{Constraints with Respect to Routing and Scheduling}

The constraints with regard to routing and scheduling are the same as the ones described in Section 5.7. However, since there is encryption delay on the sensor we need to add the following constraints.

- Initialization constraints: The messages are issued periodically in sensor $S_{i}$ according to its period $\left(h_{i}\right)$. The release time of message $m_{i}^{j}$ from $S_{i}$ is the issue time of the message plus the encryption delay:

$$
\forall i, j \mid m_{i}^{j} \in \mathscr{M}: \quad \gamma_{i S_{i}}^{j}=j \cdot h_{i}+c d_{i} .
$$

\subsubsection{Stability Constraints}

Similar to the stability-aware approach, we consider the varying end-to-end delay to calculate the stability margin. Here, the end-to-end delay $e 2 e_{i}^{j}$ is equal to the release time of that message from the last switch in the route, plus the $l d$ of the link between this switch and the controller plus the decryption delay $c d_{i}$ minus the initial issue time in the sensor.

$$
\begin{gathered}
\forall i, j \mid m_{i}^{j} \in \mathscr{M}: \text { if } \quad \eta_{i k}^{j}=C_{i}, \\
\text { then } e 2 e_{i}^{j}=\gamma_{i k}^{j}+l d+c d_{i}-j \cdot h_{i} .
\end{gathered}
$$

Therefore, the stability margin is again calculated by the Equation 5.1 and should be kept non-negative for all control applications, as shown below.

$$
\delta_{i} \geqslant 0, \quad \forall \Lambda_{i} .
$$

\subsubsection{Security Optimization}

In the security and stability-aware approach, in addition to the stability guarantees, the aim is to maximize the attack power required to break any encryption. Therefore, we define two security optimization goals. Firstly, our goal is to maximize the minimum security level among all control applications.

- Worst-Case security optimization: The minimum security level among all control applications is maximized.

$$
\underset{\text { sli }_{i}}{\operatorname{maximize}} \underset{\forall \Lambda_{i}}{\min _{i}}\left\{s l_{i}\right\} .
$$


By defining auxiliary variable $t$ we transform the equation to a standard optimization problem,

$$
\begin{aligned}
& \underset{t, s l_{i}}{\operatorname{maximize}} t \\
& \text { subject to } t \leqslant s l_{i}, \quad \forall \Lambda_{i},
\end{aligned}
$$

where the security level $s l_{i}$ can be captured using Equation 5.2. Note that the variable $t$ will contain the value of $\min \left\{s l_{i}\right\}$ after the optimization problem is solved.

Secondly, after finding the worst-case security level, the remaining resources (time) in the control applications can be dedicated to provide higher security levels. Therefore, we define another security optimization goal of maximizing the weighted sum of security levels.

- Expected security optimization: The expected security level among all control applications is maximized, on top of the minimum security level found in the worst-case security optimization (minimum $s l_{i}$ in Equation 5.22).

$$
\begin{array}{ll}
\underset{t, s l_{i}}{\operatorname{maximize}} & \sum_{\forall \Lambda_{i}} \omega_{i} \cdot s l_{i} \\
\text { subject to } & s l_{i} \geqslant t, \quad \forall \Lambda_{i},
\end{array}
$$

where $t$ is obtained in the worst-case security optimization (Equation 5.23).

In the security and stability-aware approach we also use Satisfiability Modulo Theories (SMT) to solve the problem captured by the above constraints. Moreover, we use Optimization Modulo Theories (OMT) to add the security optimization goals to the constraints and find the solution that optimizes the security objectives. SMT solvers determine the satisfiability of the above constraints but they return the first encountered satisfying assignment and not necessarily the best one. In recent years, efforts have been made to build OMT solvers by augmenting certain SMT solvers with optimization capabilities (Bjørner et al., 2015; Li et al., 2014; Sebastiani and Trentin, 2015). Using OMT-enabled tools one can add optimization goals to the problem and 
generate the optimal (and satisfying) assignments with respect to objective functions.

We first formulate the above constraints using the SMT-LIB (Barrett et al., 2010) library, then we use a solver to check their satisfiability. If the conjunction of these constraints is satisfiable, the solver associates a value to all $\eta_{i k}^{j}$ and $\gamma_{i k}^{j}$ for each message $m_{i}^{j}$ at each node $v_{k}$ of the network. In order to take into account the optimization goals, we use the SMT-LIB augmented with optimization capabilities (Bjørner et al., 2015). If we add the security optimization goals to the problem the OMT solver associates values to the same variables such that the constraints are satisfied and the optimization goals are achieved.

\subsubsection{Improving Scalability}

In Section 5.7.3, we discussed that the routing and static scheduling problems are known to be NP-complete. We also proposed two techniques to improve the scalability of the stability-aware approach and made a trade-off between the quality of synthesized solutions and the complexity of the synthesis process. In the security and stability-aware approach, we are adding two security optimizations on top of this NP-complete problem. Using built-in SMT-LIB commands to express these optimization objectives will provide optimum solutions for those problems, but it is not time efficient. In this section, we use binary search and genetic algorithms as two heuristics to improve the efficiency of solving the two optimization problems. In our experimental evaluation, we show that the heuristics can solve the scalability issue without any major loss of quality.

\subsubsection{Binary Search}

This algorithm is used to maximize the minimum affordable security level over all applications and uses the binary search technique (Cormen et al., 2001). The algorithm searches in a range of security levels from no security (no crypto overhead) to the maximum security level the encryption algorithm can provide that imposes maximum crypto overhead. The algorithm starts with the value in the middle between the minimum and maximum security level. In each iteration, for a chosen security level, the algorithm solves the basic SMT 
problem (without any optimization goal) to find out if the stability constraints are satisfied with the overheads corresponding to that security level. If the SMT solver fails to find a valid solution for the current security level, then the binary search continues with a security level between the current security level and the last security level with a valid solution from the SMT solver. On the other hand, if the SMT solver finds a valid solution for the current security level, then it continues with a security level between the current security level and the last security level with no valid solution from the SMT solver.

The binary search method is an anytime algorithm, i.e., whenever the binary search algorithm stops, it returns the best valid solution found so far, which could of course be different from the theoretical optimal solution, hence the trade-off between the time complexity and the quality of the solution that is obtained.

\subsubsection{Genetic Algorithm}

After maximizing the minimum security level, the goal is to maximize the weighted sum of security levels for all control applications. We use a genetic algorithm-based heuristic (Michalewicz, 1996) to deal with the complexity of this optimization problem. In the genetic algorithm, security level for a specific control application is considered as a gene. Therefore, the set of security levels of control applications are captured as a chromosome. We initialize the population by several chromosomes with security levels that satisfy the basic SMT problem. The basic SMT problem inside the genetic algorithm contains an additional constraint so that the security level of all applications has to be larger or at least equal to the one produced by the binary search. The fitness function is the weighted sum of the security levels, which we would like to maximize (see Equation 5.24). For each iteration of the genetic algorithm, we select chromosomes with best fitness levels from the population and then we use the traditional mutation and cross-over operations to get a new chromosome (generation). Then we run a basic SMT problem based on the security levels from this new chromosome. If the SMT problem was not satisfied we discard the chromosome otherwise we add the chromosome to the population. The genetic algorithm is run for a fixed number of iterations (generations). The chromosome in the final population with the best fitness level is the se- 


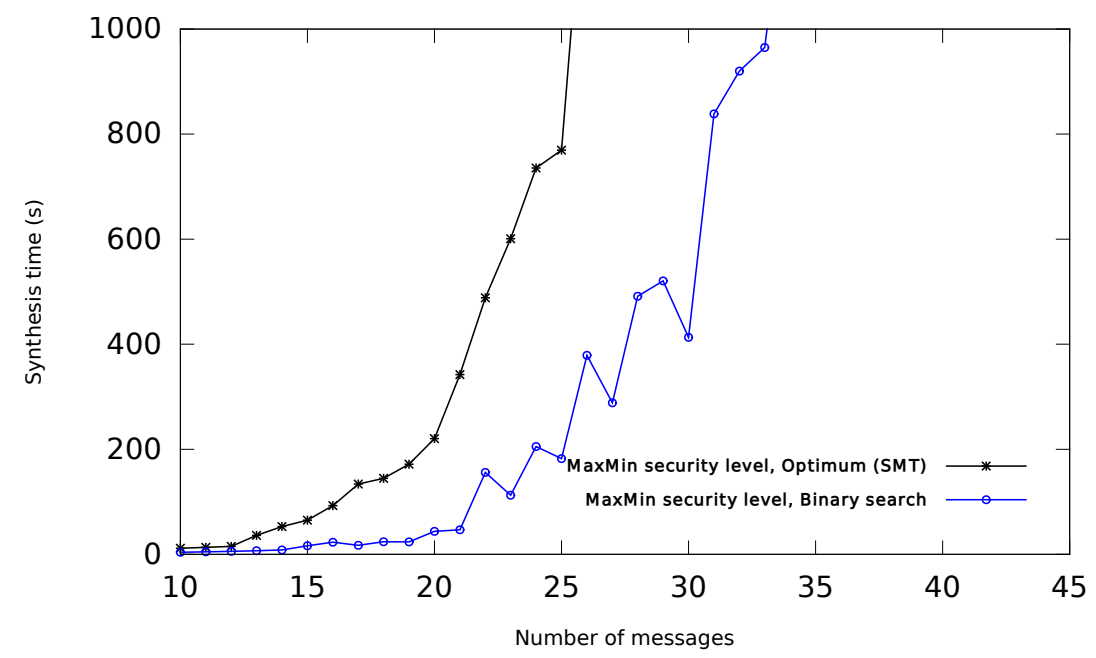

Figure 5.10: Synthesis time for theoretical optimum and binary search.

lected answer which maximizes the weighted sum of security levels for control applications.

Finally, note that the binary search and genetic algorithms can easily be used together with the two heuristics we proposed in Section 5.7.3 to solve the routing and scheduling scalability issue. Therefore, the basic SMT problem that is used inside the binary search and genetic algorithm can use the route subset and incremental synthesis heuristics for efficiency.

\subsubsection{Experimental Setup and Results}

In this section, we experimentally evaluate the efficiency of our proposed security and stability-aware routing and scheduling technique. Our experiments are carried out using the Z3 (De Moura and Bjørner, 2008) SMT and OMT solver. They were run on a regular desktop computer with $2.67 \mathrm{GHz}$ Xeon CPU and 6 GB of RAM. For the first three experiments, we randomly choose control applications from a database with inverted pendulums, ball and beam processes, DC servos, and harmonic oscillators. Finally, we evaluate our approach with a real-life case study similar to the one used in Section 5.7.4. 


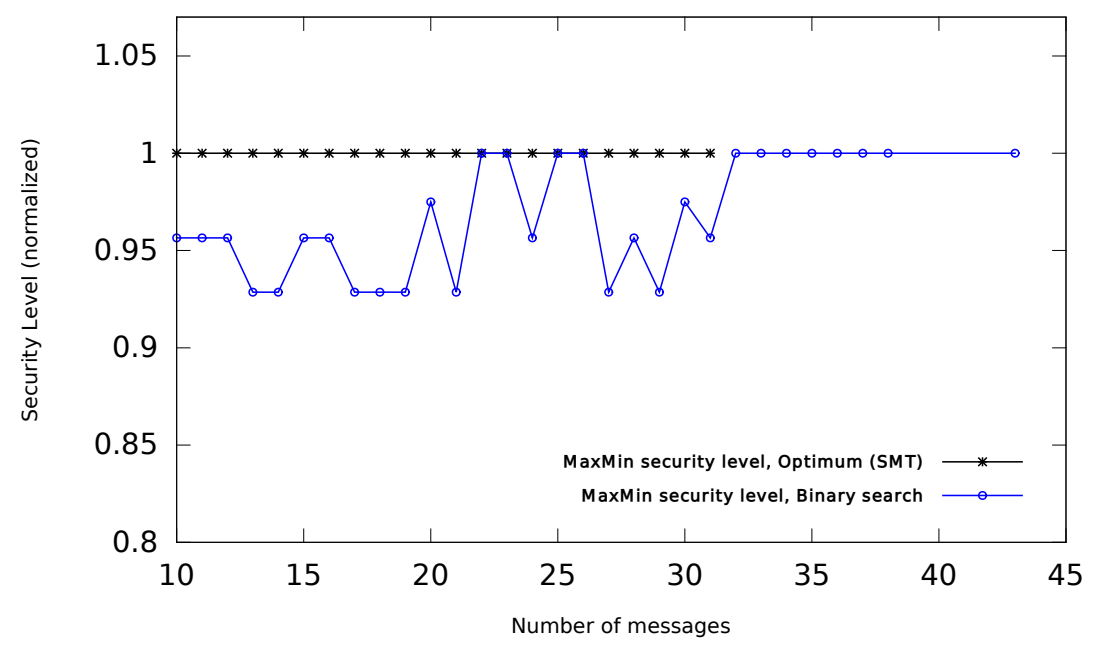

Figure 5.11: Maximum of the minimum security level for the theoretical optimum and binary search.

In the first experiment, we consider the first optimization problem (maximizing the minimum security level) and solve the SMT problem without any heuristics with regard to finding routes and schedules. But for the optimization goal, we run the experiment twice: once using the built-in SMT-LIB optimization and once using binary search. In this experiment, we run 45 different synthesis problems on different networks, all with 23 nodes (4 sensors, 4 controllers, and 15 Ethernet switches). For each synthesis problem, the 4 control applications are chosen randomly, as discussed previously. Figure 5.10 and Figure 5.11 show the synthesis time and security level versus number of messages, respectively. These figures compare the optimum (built in SMTLIB) and binary search solutions. The binary search (the blue curves) is run for 4 iterations and takes less time than the optimum solution (the black curves). Figure 5.11 shows the accuracy of the binary search solution (blue curve) compared to the optimum solution (black curve). According to this figure, the binary search is always more than $90 \%$ accurate. The timeout for running the problems was 3000 seconds, hence the optimum security level for some of the experiments is not reported (see Figure 5.11). Note that even with the binary search, the synthesis time is more than 3000 seconds when the number of messages is more than 42. Accordingly, the provided solu- 


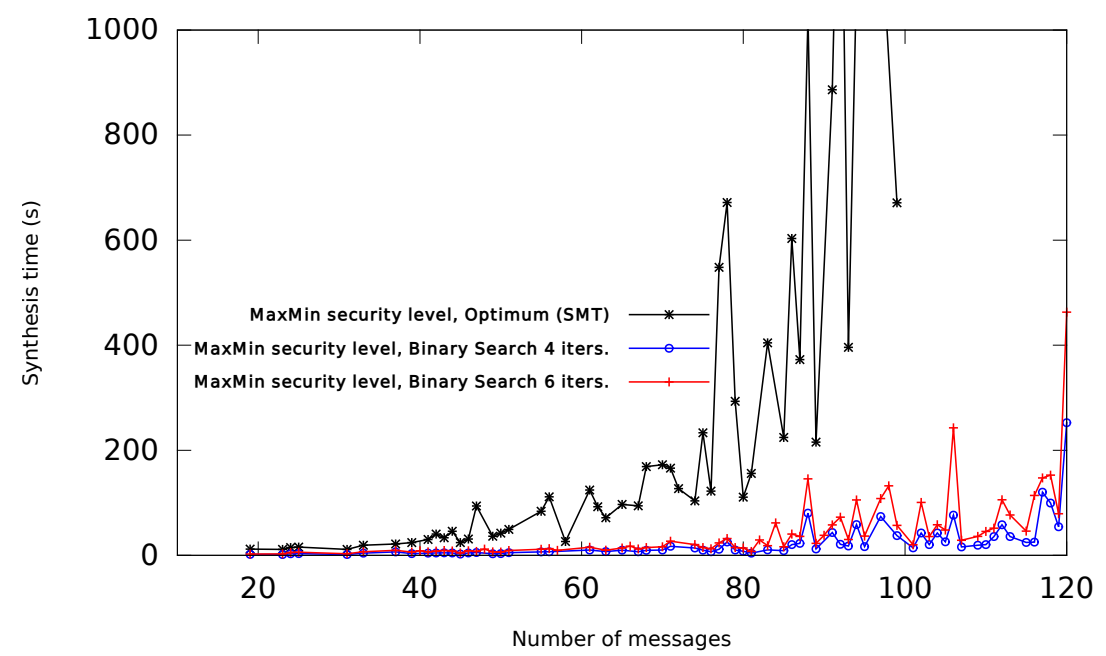

Figure 5.12: Synthesis time for SMT-LIB optimization and binary search with heuristics (routes $=4$, slices $=5$ ).

tion has scalability issues and is not efficiently applicable for bigger systems with large number of messages. Thus, in the following experiments, we add the two heuristics (route subset and incremental synthesis) to the basic SMT formulation.

In the second experiment, we again consider the first optimization problem (maximizing the minimum security level), but this time we use the heuristics in the basic SMT formulation, making it possible to run the experiment for much larger number of messages. We have applied our heuristics providing 4 alternative routes for each message and considering 5 time slices. In this experiment, we run 60 different synthesis problems on different networks, all with 35 nodes (10 sensors, 10 controllers, and 15 Ethernet switches). For each synthesis problem, the 10 control applications are chosen randomly, as discussed previously. For the optimization goal we compare the two solutions, once using the built-in SMT-LIB optimization and once using the binary search. Figure 5.12 and Figure 5.13 show the synthesis time and security level for the optimum (built in SMT-LIB) and binary search solutions. For the binary search we run the problem once with 4 iterations (the blue curves) and once with 6 iterations (the red curves), and both take less time than the 


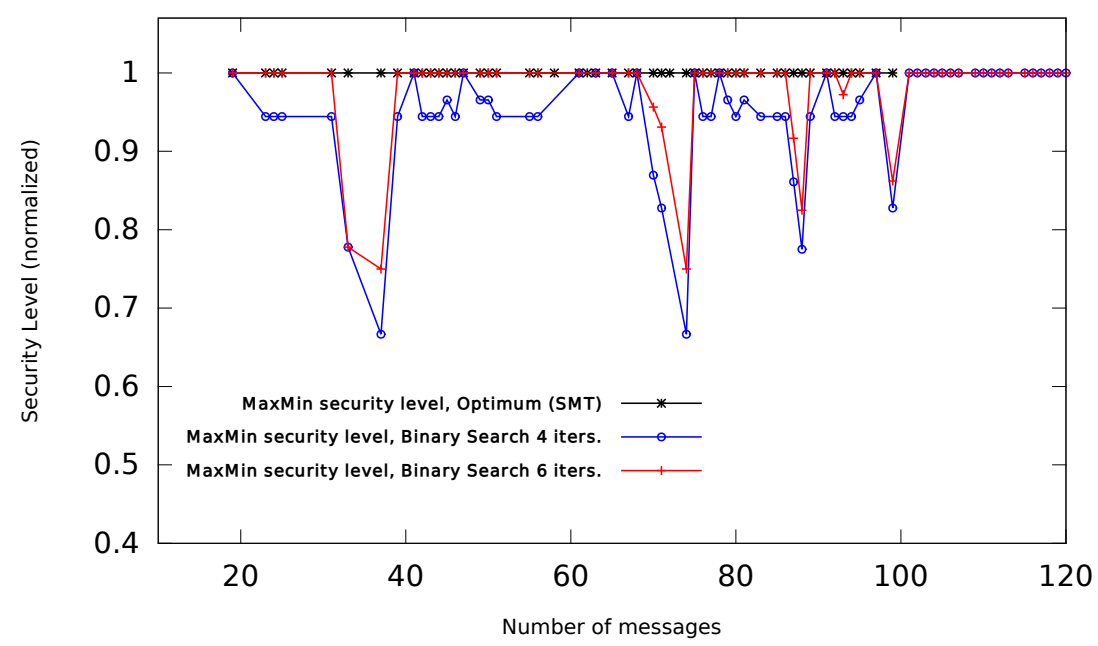

Figure 5.13: Maximum of the minimum security level for SMT-LIB optimization and binary search with heuristics (routes $=4$, slices $=5$ ).

optimum solution (the black curves). According to Figure 5.13, the binary search finds the optimum security level with reasonable accuracy. Moreover, the binary search with 6 iterations provides more accurate security levels with a small increase in synthesis time. Note that the timeout for running each experiment is 3000 seconds. For the built-in SMT-LIB solution, the timeout is reached when the number of messages is around 100, while the binary search provides solutions for large problems. Hence, the optimum security level is not reported for the experiments with more than 100 messages.

After maximizing the minimum affordable security level, the goal is to further maximize the weighted sum of the security levels of all control applications. For this optimization goal, we again compare the two solutions: once using the built-in SMT-LIB optimization and once using a genetic algorithm. In this experiment, we again run 60 different synthesis problems on different networks with 35 nodes (10 sensors, 10 controllers, and 15 Ethernet switches). For each synthesis problem, the 10 control applications are chosen randomly, as discussed previously. In the basic SMT problem, we have applied our heuristics providing 4 alternative routes and 5 time slices. Figure 5.14 shows the synthesis time for the optimum (built in SMT-LIB) and genetic algorithm 


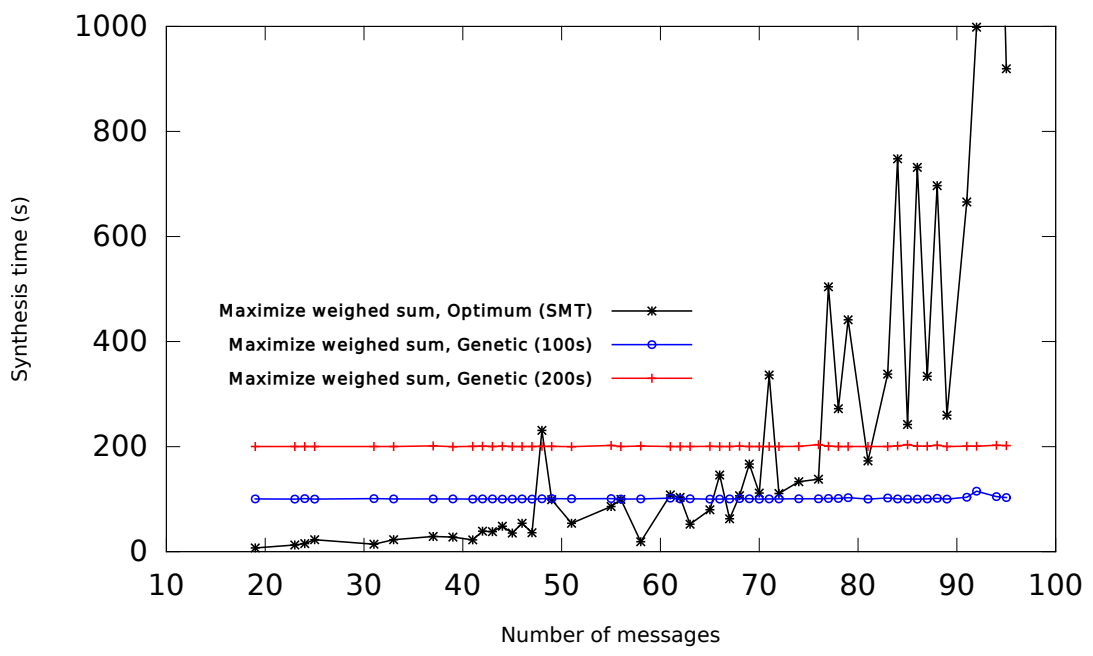

Figure 5.14: Synthesis time for SMT-LIB optimization and genetic algorithm with heuristics (routes $=4$, slices $=5$ ).

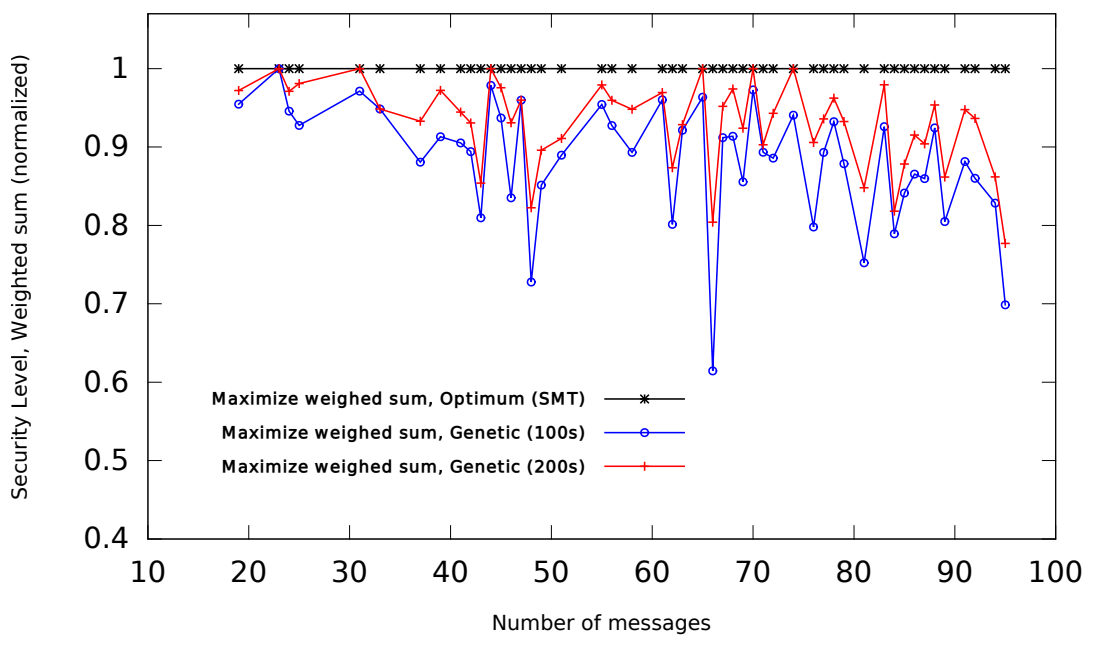

Figure 5.15: Maximum weighted sum for SMT-LIB optimization and genetic algorithm with heuristics (routes $=4$, slices $=5$ ). 
solutions. For the genetic algorithm, we run the program first with limiting the optimization time to 100 seconds (the blue line) and a second time with a limit of 200 seconds (the red line). Figure 5.15 shows the accuracy of the results obtained with the genetic algorithm, relative to the ones produced with SMT-LIB. The red and blue lines show the results for running the genetic algorithm for 100 and 200 seconds, respectively. The figures show that the genetic algorithm is efficient for the problem, even with larger numbers of messages.

Finally, we have also applied our security and stability-aware approach to the real-life example discussed in Section 5.7.4 with the same setup. The parameters (period, $\alpha$, and $\beta$ ) for five of these control applications are shown in Table 5.9.

We assume that the sensors and controllers are using the RC6 algorithm with 256 bit key size and 50 different numbers of rounds from 2 to 100 . Therefore, different security levels and encryption/decryption times are assumed for messages. Considering that the sensors and controllers are using the same processor as the one used in Section 5.2.1, then from Figure 5.3 one can obtain these 50 different crypto overheads for different numbers of rounds for RC6 with 256 bit key size. The synthesis problem for our set of 20 applications comprises the scheduling and routing of 106 messages (generated in a hyperperiod of length $200 \mathrm{~ms}$ ). We have applied our synthesis approach providing 3 alternative routes for each message and considering 6 time slices.

The first optimization goal is to maximize the minimum security level. Thus, we run this experiment twice, once using the built in SMT-LIB optimization that will find the optimum solution and once using the binary search with 5 iterations.

The synthesis time to find the optimum solution (using the built-in SMT-LIB) was 195 minutes, and an encryption/decryption with $r=56$ rounds maximizes the minimum security level for all control applications. The provided routes and schedule satisfy the worst-case stability constraints for all 20 applications. The resulting maximal end-to-end delay, latency, and jitter for five out of 20 control applications are shown in Table 5.9 under the Optimum column. 
We also run the synthesis problem using the binary search algorithm with 5 iterations. The synthesis time to find the solution this time is 72.44 seconds and $r=54$ rounds is provided as the optimum number of rounds that maximizes the minimum security level. The result again satisfies the stability constraints for all applications. The result for five of the applications is shown in Table 5.9 under the Binary column. 


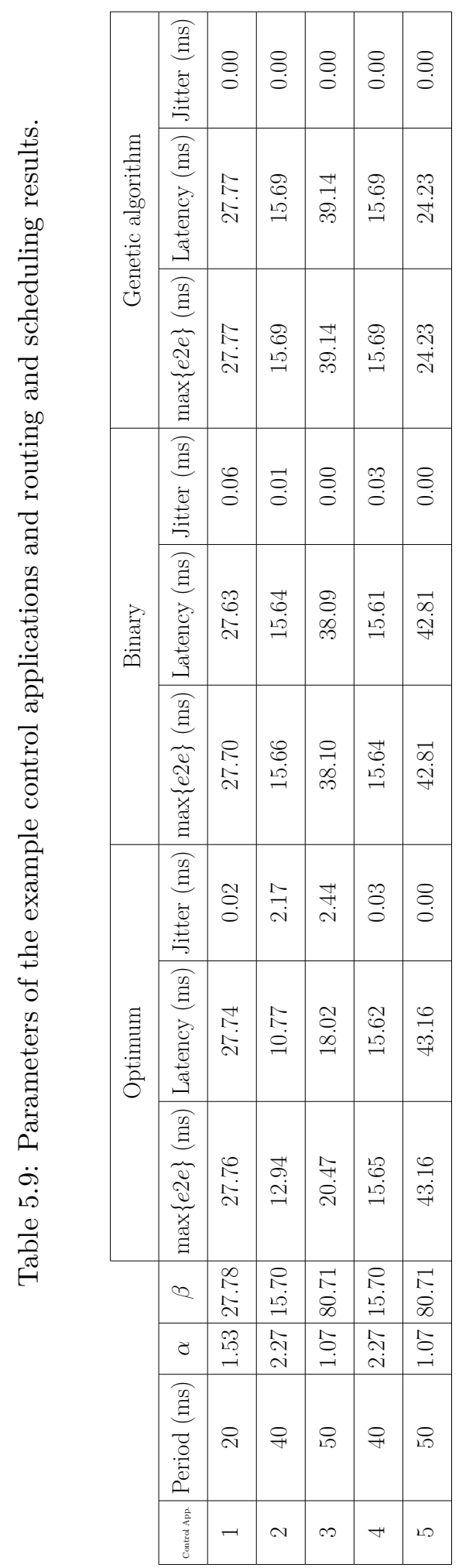


To further maximize the security level, we also run the example with the second optimization goal (maximizing the weighted sum). For this goal, we have also run the experiment twice: once using the built-in SMT-LIB optimization and once using the genetic algorithm with optimization time limited to $200 \mathrm{~s}$. The built-in SMT-LIB optimization did not converge to a solution, even after 24 hours, hence we only report the result from the genetic algorithm. The genetic algorithm provides a solution that satisfies the stability constraints and guarantees minimum security level (captured by encryption/decryption with $r=56$ rounds) for all applications. The results from the genetic algorithm show that the security level for 19 out of 20 applications were above the minimum security level and 18 out of 20 control applications were provided with the security level captured by encryption/decryption using $r=80$ rounds. The resulting maximal end-to-end delay, latency, and jitter for five out of 20 control applications are shown in Table 5.9 under the Genetic algorithm column. 


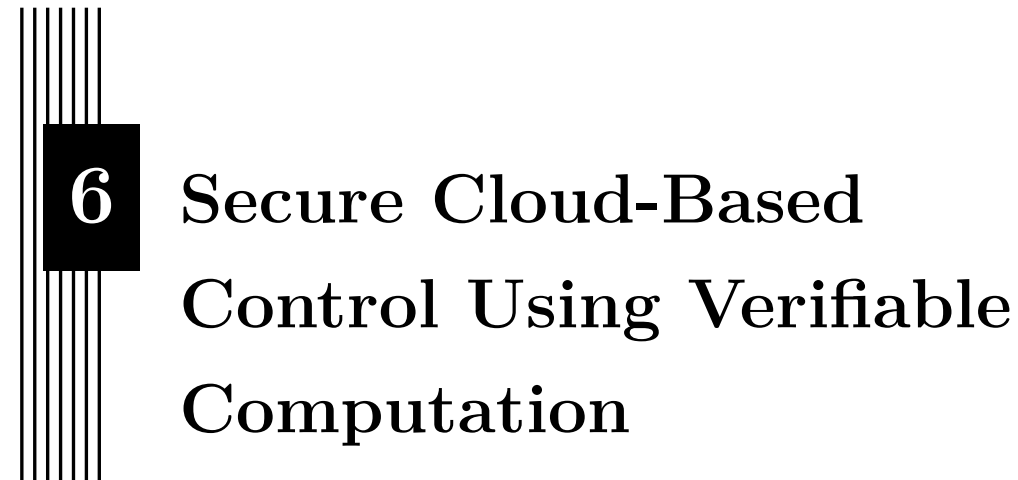

While outsourcing control applications to the cloud is trending, one of the biggest challenges is ensuring the safety and security of the system. Security can be provided for critical control applications by equipping the physical plant with a mechanism that can verify the control signal received from the cloud. The verification process should ensure that the signal has not been manipulated by an attacker or miscalculated by the cloud. Recent work in the field of modern cryptography has led to the development of verifiable computation techniques. Such techniques allow a client to verify the correctness of a remote execution.

In this chapter, we investigate RQ4 (see Section 1.2). We deliver a proof of concept for the applicability of a verifiable computation scheme to control applications. In our experiments, we showcase the practicality of verifiable computation for two different physical plants with different timing demands. We also deliver a real-life example using a water tank system as the client and Microsoft Azure Cloud services as the cloud platform. Our experiments demonstrate the effectiveness of the verifiable computation scheme for secure cloud-based implementations of advanced control methods, such as Model Pre- 
dictive Control (MPC). The content of this chapter is based on the following publication: (Mahfouzi et al., 2021).

\subsection{Introduction and Related Work}

Recently, cloud computing platforms have attracted a lot of attention in the control and cyber-physical systems domains, with a growing number of projects being developed across many industries. Prominent examples of potential application areas are autonomous driving and industrial automation. There are a number of reasons for the adoption and application of cloud-based solutions.

Many CPSs are witnessing fast-growing market demands for features and functions implemented in software. From a maintenance and upgrade perspective, there is a clear trend toward outsourced control software on a cloud server rather than implementation on dedicated embedded Programmable Logic Controllers (PLCs) (Inaltekin et al., 2018; Papcun et al., 2018; Pei Breivold, 2020; Shahraeini et al., 2010). For example, the process of maintaining and updating the on-board controller for every turbine in a wind power farm is complex and error-prone, but this is no longer the case when the controller is implemented on a cloud server. Examples can also be found in the automotive industry, where an Original Equipment Manufacturer (OEM) provides feature improvements to millions of vehicles. In the automotive case, there is also a demand for outsourcing of the controller to a virtual Electronic Control Unit (ECU) in the Control as a Service (CaaS) context (Esen et al., 2015). Another driving force toward the adoption of cloud computing in CPSs is third-party software integration. There is a growing number of instances in which, due to intellectual property issues, one or more third-party vendors are not comfortable with executing their software on millions of embedded platforms.

In addition to significantly reducing the complexity of maintenance and enabling third-party software integration, cloud computing is a promising solution in terms of scalability, flexibility, and agile development. Outsourcing a controller as a software executing on a cloud server makes the system adaptable, agile, and flexible (Givehchi et al., 2014, 2013). Features such as 
Function as a Service (FaaS) and cloud-native programming reduce development effort and production costs (hardware and associated labor) (Hegazy and Hefeeda, 2014), as well as time to market (Pelle et al., 2019). Many embedded systems have limited local computing and energy capacity. Cloud providers, however, offer a variety of services on cloud servers with enormous computational power, which is often required for complex control algorithms and machine-learning techniques (Årzén et al., 2018; Skarin et al., 2020). Finally, many complex CPSs are inherently distributed (e.g., power grids, industrial automation, and autonomous driving), where the sensor data is collected from multiple locations. Cloud platforms are a natural host for software that integrates data from various sources.

Control systems usually comprise multiple critical physical systems. Outsourcing of control computation and software to a cloud platform increases exposure to security attacks due to new attack surfaces (wireless communication interfaces and cloud platform) (Subashini and Kavitha, 2011), which could have catastrophic consequences. The confidentiality of the messages to/from the untrusted outsourced controller has been discussed in the literature (Alexandru et al., 2018; Farokhi et al., 2017; Kim et al., 2016; Kogiso and Fujita, 2015). The proposed solutions use a suitable homomorphic encryption scheme to run the controller on encrypted data. The authors in (Alexandru et al., 2020, 2018; Darup et al., 2017) use homomorphic encryption to outsource an MPC controller to the cloud. The homomorphic encryption enables the cloud service to receive encrypted input from the client and calculate the encrypted output without needing to decrypt the private input. These efforts guarantee the confidentiality of the messages being transmitted to/from the cloud but they do not ensure the integrity of these messages, which is the main concern of this chapter.

The classical authentication and integrity issues have been widely discussed in the literature and can be solved with appropriate encryption techniques (Jutla, 2001). The applicability of such solutions in the context of real-time and control systems with stringent resource constraints has been well studied (Aminifar et al., 2017; Lesi et al., 2017), with consideration given to performance degradation as a result of implementing encryption techniques (Mahfouzi et al., 2019a). We have addressed this aspect in Chapter 5. In (Cheon et al., 2018), the authors propose the use of real-time homomorphic authenticated 
encryption to secure a drone with an outsourced controller against eavesdropping and forgery attacks. However, these solutions assume that the cloud is reliable and that the client can trust the response provided by the remote computation.

Concerns about untrusted cloud servers have led to three approaches to verifying the correctness of the response provided by the cloud. (1) Verification by replication is used in several distributed computation projects (Anderson et al., 2002) and exploits identical responses received from several replica of the same computation to verify the correctness of the computation. (2) Periodic auditing and testing by a third party can be used to check the correctness of results from the cloud (Bouchenak et al., 2013; Tian et al., 2015). (3) Verifiable computation provides mathematical proof that guarantees the integrity of data received from the cloud (Walfish and Blumberg, 2015). While the three aforementioned approaches all solve the problem of untrusted cloud servers and manipulated communication channels altogether, the two former solutions do not provide a guarantee on the integrity of the data and are not suitable for critical control applications. Moreover, to the best of our knowledge, none of the above approaches has been studied in the context of control systems. Therefore, we focus on the effectiveness of the verifiable computation approach for control applications.

Verifiable computation protocols consist of a verifier (client) and a prover (cloud). The prover creates mathematical proof to demonstrate the correctness of the results that are returned to the verifier. Therefore, using verifiable computation, the correctness of the output from the cloud can be guaranteed, which allows the client to ensure that the data and the software execution itself have not been manipulated by attackers. There are, however, two main challenges that restrict the applicability of current verifiable computation techniques: (1) The limitations imposed by current experimental verifiable computation frameworks with regard to the class of computations they can handle (allowed arithmetic operations, data and control structures accepted, etc.); (2) Their computational complexity, which implies potentially heavy calculations, especially for the proof generation in the cloud. This is particularly important in the context of control applications that are sensitive to time delays (Aminifar et al., 2015a,b, 2012; Bini and Cervin, 2008; Lincoln and Cervin, 2002; Mahfouzi et al., 2019b, 2018; Samii et al., 2009; Seto et al., 
1996). While theoretically appealing, the practical applicability of verifiable computation is still an open question. More specifically, the possible utilization of this technique for secure cloud deployment of control tasks has not yet been addressed in the literature.

This chapter delivers a proof of concept for the applicability of the verifiable computation scheme to control applications. In our experiments, we showcase the practicality of verifiable computation on two different physical plants with different timing demands. We also deliver a real-life example on a water tank system that uses an MPC controller executing on Microsoft Azure Cloud services. Our experiments show the effectiveness of the verifiable computation scheme for the secure cloud-based implementation of advanced control methods. Our main contributions are as follows:

- implementation and proof of concept of an industrial controller in a cloud-based verifiable computation framework;

- determination of the degradation in control performance as a result of introducing verifiable computation as a security mechanism over the cloud;

- evaluation of the performance and control cost of different control applications and different control parameters (e.g., prediction horizon and sampling period), in a cloud-based verifiable computation framework.

\subsection{Verifiable Computation}

Recent studies and advances in the area of verifiable computation show substantial improvement, indicating the possiblility of future deployment in practice (Costello et al., 2015; Fiore et al., 2020; Parno et al., 2013). Verifiable computation, in general, involves three parties: a verifier (client), a trusted third party, and a prover (cloud). The trusted third party outsources a function to the cloud. The client then sends an input to the cloud that is hosting the outsourced function. The cloud calculates the outsourced function on the input and sends back the output with a mathematical proof to the client. The client then is able to verify that the output provided by the cloud is indeed the output of the function computed on the input. In many cases, if the out- 
sourced function is not confidential to the client the trusted third party and the verifier (client) are the same.

We use Pequin (Howald and al., 2019), which is an efficient implementation of a verifiable computation scheme. The verifiable computation scheme considered in Pequin is formally defined with the following three algorithms:

- $\left(P K_{F}, V K_{F}\right) \leftarrow K e y G e n(F)$ : The KeyGen algorithm takes the function $F$ to be outsourced as an input. It generates proof key $P K_{F}$ and verification key $V K_{F}$. This algorithm is run by a trusted third party. The proof key $P K_{F}$ is then stored in the cloud and the verification key $V K_{F}$ is stored at the client. The KeyGen function will run only once at the installation.

- $\left(u, \pi_{u}\right) \leftarrow$ Compute $\left(P K_{F}, y\right)$ : The Compute algorithm takes the proof key $P K_{F}$ and input $y$ and generates $u=F(y)$ and a proof $\pi_{u}$ showing the correctness of $u$. This algorithm is run by the cloud for each input received from the client.

- $\{0,1\} \leftarrow \operatorname{Verify}\left(V K_{F}, y, u, \pi_{u}\right)$ : The Verify algorithm takes the verification key $V K_{F}$, input $y$, purported output $u$, and proof $\pi_{u}$. It outputs 1 if $F(y)=u$ and 0 otherwise. This algorithm is run by the client for each output received from the cloud.

This scheme guarantees the following characteristics:

- Correctness: For every function $F$ and input $y$ if we run $\left(P K_{F}, V K_{F}\right) \leftarrow \operatorname{KeyGen}(F)$ and $\left(u, \pi_{u}\right) \leftarrow \operatorname{Compute}\left(P K_{F}, y\right)$, then we always get $\operatorname{Verify}\left(V K_{F}, y, u, \pi_{u}\right)=1$.

- Security: For any function $F$, if $F(\hat{y}) \neq \hat{u}$ then the probability that an adversary finds $\hat{\pi}_{u}$ such that $1=\operatorname{Verify}\left(V K_{F}, \hat{y}, \hat{u}, \hat{\pi}_{u}\right)$ is negligible ${ }^{1}$.

- Zero-Knowledge: The client learns nothing from the cloud other than the output. This means that the function $F$ is kept confidential from the client, if a trusted third party runs the KeyGen algorithm.

\footnotetext{
${ }^{1}$ Adversaries can potentially succeed with extremely small probability, small enough that we are not concerned that it will ever really happen (Katz and Lindell, 2014).
} 
State-of-the-art verifiable computation frameworks, including the Pequin toolchain, impose strong implementation limitations in terms of the allowed arithmetic operations, data and control structures accepted, etc. We study these technical limitations and our implementation approach to overcome them in Section 6.5. Our intention in this chapter is to show the applicability of these frameworks by using them to secure an MPC controller (see Section 2.4) that has been outsourced to a cloud platform.

\subsection{System Model}

We consider the system depicted in Figure 6.1. We use the MPC framework described in Section 2.4 to control the physical plant. A trusted third party outsources the MPC controller to the Microsoft Azure cloud. In each sampling period, the local controller receives the plant output from the physical plant and sends it to the cloud. The cloud then runs the computation (MPC) with the received input and calculates the control signal. The cloud also encodes a proof and sends the proof along with the control signal to the local controller. The local controller then checks the proof and verifies the correctness of the received output, after which the actuation is performed.

Using the verifiable computation scheme the following security measures are established:

- the client can detect manipulation in the communication channel and miscalculated/forged data from the cloud, hence, it can verify the integrity of the received data;

- the client learns nothing about the outsourced computation itself, e.g., proprietary control policies.

We assume that an assisting controller is locally available as an emergency mechanism in case the received data is not verified or the communication to the cloud is broken. This mechanism facilitates the graceful and safe degradation of normal system operation, e.g., using a local control approach and/or raising an alarm. 


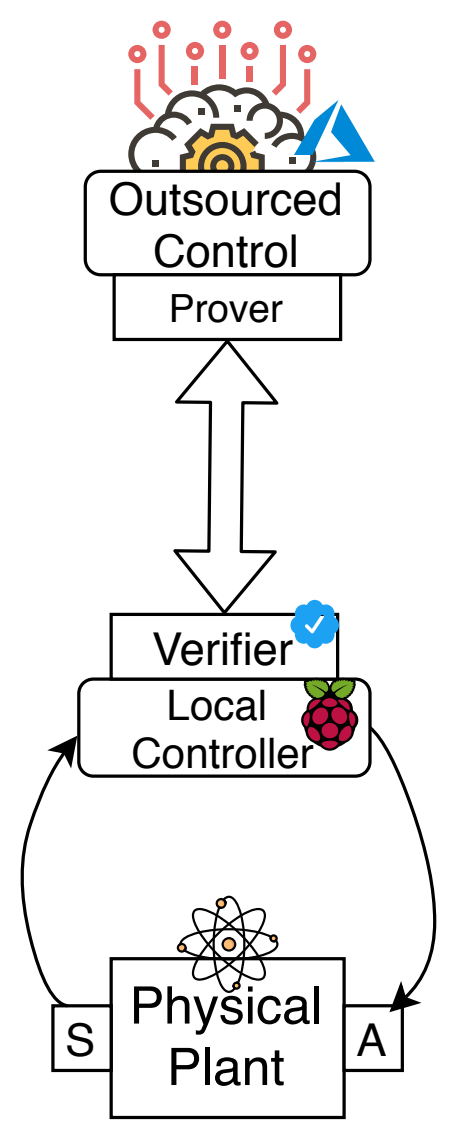

Figure 6.1: Overview of the outsourcing scenario.

The delay imposed by applying verifiable computation on cloud control, which we call round-trip time in this chapter, includes (1) communication time to and from the cloud, (2) time spent in the cloud to calculate the output and generate the proof, and (3) time spent on the client to perform verification. The round-trip time should be such that the physical plant is kept stable.

\subsection{Threat Model}

In the outsourcing scenario depicted in Figure 6.1 the threats faced by the local controller come either from malicious behavior in the cloud or from possible 
manipulation of the communication channel between the local controller and the cloud. We assume:

- for any query, the cloud may try to cheat the local controller by providing a wrong response,

- the data transmitted to/from the cloud might be forged in the communication channel, and

- the local controller is interested in learning the control algorithm (owned by the service provider) that has been outsourced to the cloud.

Note that preserving the confidentiality of messages transmitted between the local controller and the cloud is orthogonal to this work and is solved in the literature.

\subsection{Implementation of MPC using Pequin}

As discussed earlier, state-of-the-art verifiable computation frameworks, including the Pequin toolchain, impose certain limitations for implementing the function to be outsourced. In this section, we illustrate the limitations of the Pequin toolchain and explain how we implement the MPC controller using this tool.

For the Pequin toolbox, the function to be outsourced to the cloud must be written as a $\mathrm{C}$ program. The Pequin toolchain takes the $\mathrm{C}$ program and transforms it to a set of arithmetic constraints. These constraints are satisfiable if and only if the purported output (which the prover will produce) is what the original program would have produced. In Pequin, the prover side uses a probabilistic proof protocol (Goldreich, 2008) to generate a proof that convinces the verifier that the constraints are indeed satisfiable (if they are); the verifier checks the encoded proof and is able to detect if the constraints are not satisfiable. However, there are several limitations when it comes to implementing the C program. Pequin, like other implementations of the verifiable computation scheme, has a built-in compiler that allows the developer to express a computation in a restricted subset of C. For example, the latest Pequin version does not support floating point variables. This makes the implementation of the outsourced function more challenging. 
In order to support the floating point variables in our implementation we use fixed-point arithmetic with $10^{-5}$ accuracy. In the cases where the controller is designed based on floating point variables, like the case studies and the reallife example in the next sections, we use a converter in the local controller that multiplies the plant outputs by $10^{5}$ and then converts the floating point values to $64 b i t s$ integer values and then sends them to the cloud. We also convert the control signal received from the cloud to floating point by dividing it by $10^{5}$ in the local controller.

Using the fixed-point arithmetic reduces the accuracy and control performance of the outsourced controller. However, as our experiments in the following sections demonstrate, the outsourced controller implemented using Pequin performs as expected. In addition, improving the implementation of the verifiable computation frameworks is an active operation (Blumberg et al., 2021), hence these implementation limitations are expected to be resolved in the future.

\subsection{Case Studies}

In this section, we study two control systems with different timing demands and show how verifiable computation affects the control performance. The helicopter system has fast dynamics and demands smaller sampling periods to stabilize, while the water tank system can tolerate longer periods. Our experiments show that verifiable computation is applicable even with the stringent timing constraints of the helicopter case. Moreover, our experiments suggest that in the case of the helicopter system, a more powerful cloud server is needed in order to reduce the proof generation time in the cloud and maintain the timing demands of the system.

\subsubsection{Case 1: Helicopter System}

The three-degrees of freedom (3-DOF) helicopter system shown in Figure 6.2 is an example of a control system with fast dynamics used in educational and research projects (Quanser Inc., 2019a). In this system, the main arm is connected to a base that allows the arm to rotate at different angles. The elevation angle $\theta_{e}$ measures the angle of the arm relative to the horizontal plane, while the rotation angle $\theta_{r}$ measures the rotation of the arm around 


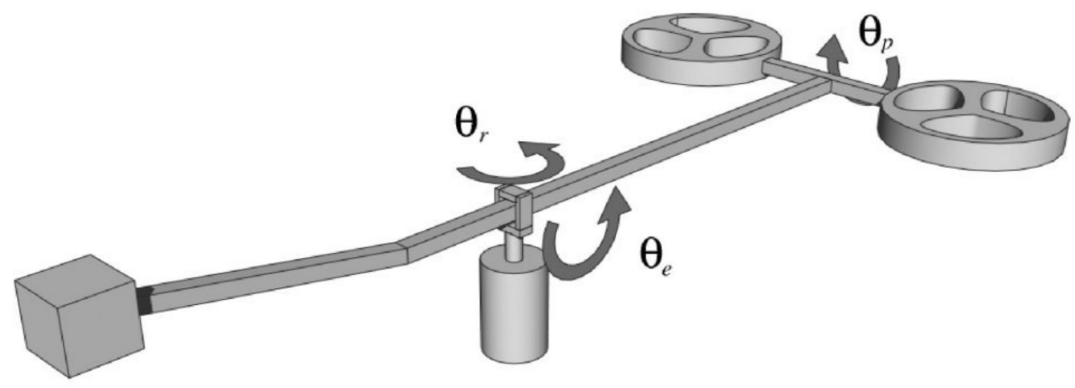

Figure 6.2: Schematic of the helicopter system (Åkesson, 2006).

the base. There is a counterweight on one side and two propellers connected to a bar on the other side. The pitch angle $\theta_{p}$ measures the angle of the bar with two propellers around the arm axis. The control input signals are the two voltages fed to the propeller motors. The three angles $\left(\theta_{e}, \theta_{r}, \theta_{p}\right)$ capture the state of the plant. The controller should control the elevation and rotation angle while maintaining the constraint that the pitch angle must not be too large. In Section 2.4 we illustrated the MPC approach to controlling a dynamic system. The dynamic model of the helicopter system can be written in the form of Equation 2.6 and the constraints in the form of Equation 2.7. Therefore, an MPC controller can be designed to control this helicopter system where the cost function is in the standard format as represented in Equation 2.8. Subsequently, we outsource the controller to the cloud and use verifiable computation to preserve the integrity of the control input signals.

In this experiment, the sampling period for the MPC controller is set to 1 s and the prediction horizon is 30 steps. The controller (empowered by verifiable computation) is outsourced to the Microsoft Azure cloud platform. In the experiment, we change the desired state of the system at time $0 s$ so that the reference elevation angle is $0.3 \mathrm{rad}$ and the reference rotation angle is $3 \mathrm{rad}$. Figure 6.3 shows how the helicopter system performs in the secure outsourcing scenario and how the rotation and evaluation angles converge to the reference values. The Azure cloud service uses an AMD EPYC 7452 processor and 2 virtual CPUs (costs 0.036 USD per hour). This setup provides us with an 
6. Secure Cloud-Based Control Using Verifiable Computation
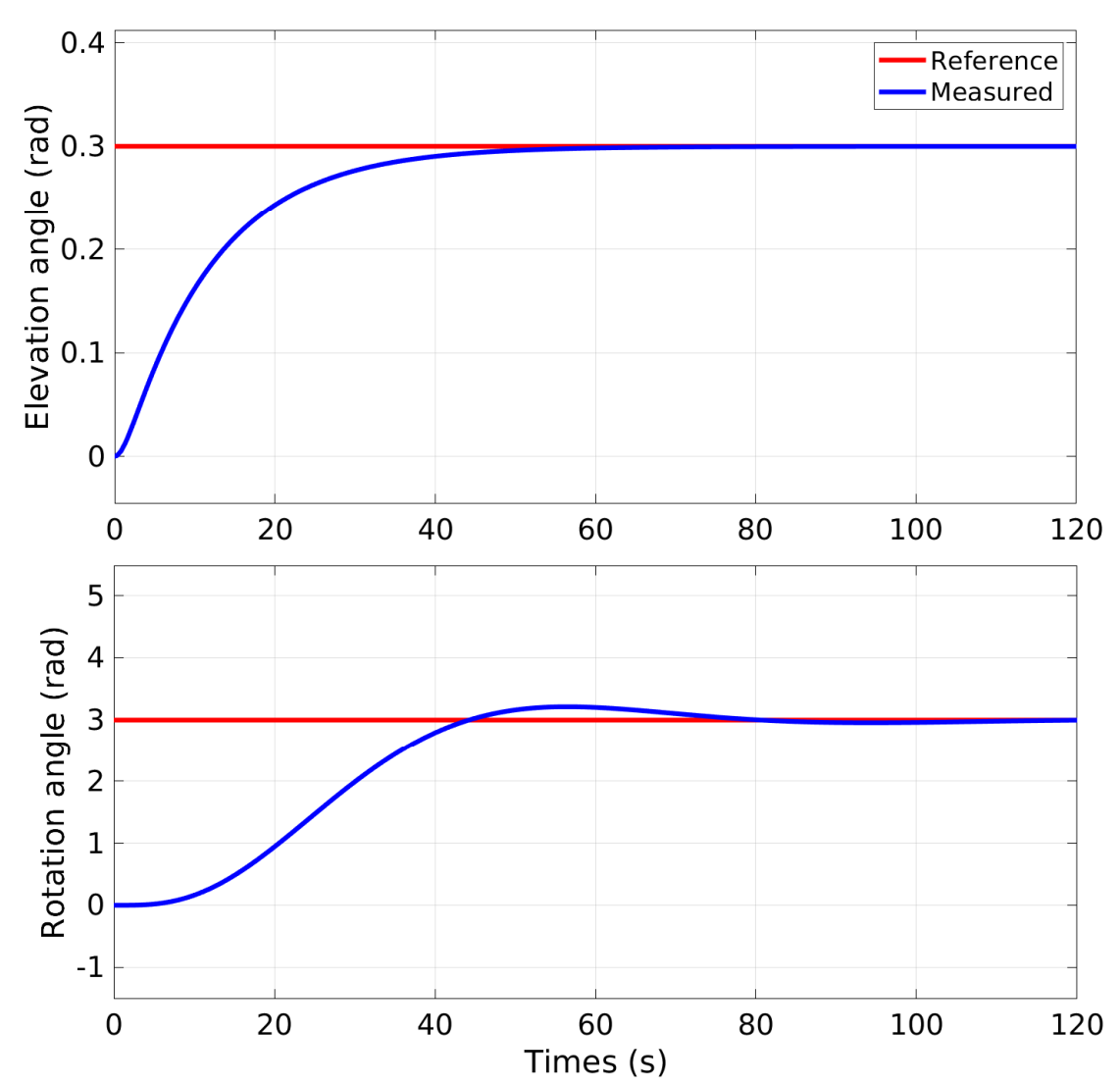

Figure 6.3: The elevation and rotation angles. The red lines show the reference trajectory and the blue curves show the actual angles.

average round-trip delay (from the sensor to the actuator) of $0.732 \mathrm{~s}$ (less than the sampling period, i.e., 1s). Figure 6.4 separately specifies different parts of the round-trip delay.

\subsubsection{Case 2: Water Tank System}

The water tank system is an example of physical plants with larger (a couple of seconds) sampling periods (Johansson, 2000). The overview of the water tank system is depicted in Figure 6.5. The system is used in various educational and 


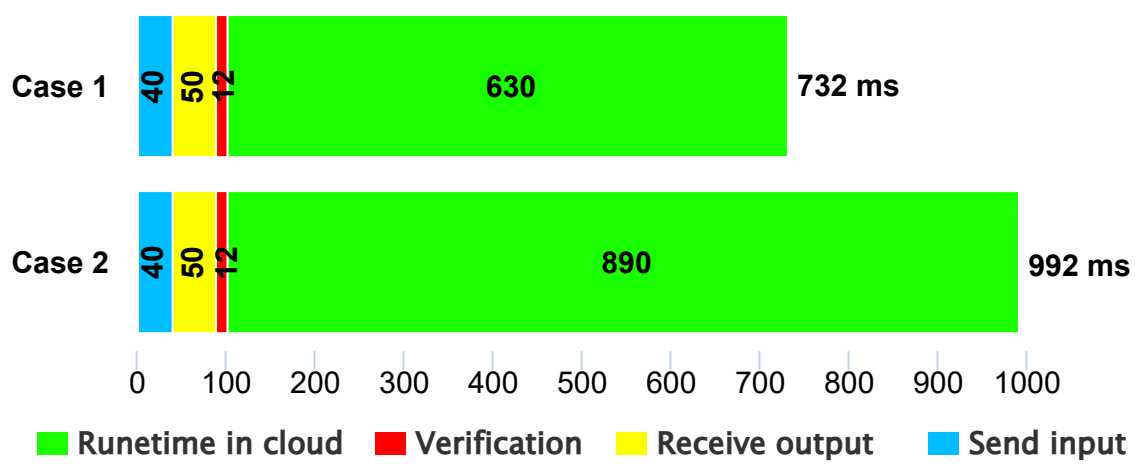

Figure 6.4: Round-trip time for helicopter (case 1) and water tank (case 2 ) system. The blue and yellow boxes (two left boxes) in each bar show the communication time. The red boxes show the verification time on the client and the green boxes show the time spent in the cloud to calculate the control signal and generate the proof. The reported times are the average of 1000 repetitions of the experiment and the standard deviation for sending input and receiving output (communication time) is $1 \mathrm{~ms}$. All reported times are in ms.

research projects to represent industrial control systems like petrochemical plants and water level control facilities.

The water tank system consists of four water tanks and two pumps. Water from the two upper tanks, $A 1$ and $B 1$, flows to the two lower tanks, $A 2$ and $B 2$, respectively. The first pump pours water into the upper right $(B 1)$ and lower left $(A 2)$ tanks, and the second pump pours water into the upper left $(A 1)$ and lower right $(B 2)$ tanks. There is a valve for each pump that sets the proportion of water going to the upper and lower tanks, and we assume that this proportion is fixed and given in this system.

The controller controls the voltage of the two pumps. The goal is to keep the water level in the two lower tanks, $A 2$ and $B 2$, at a certain level while avoiding overflow of water in any of the four tanks. The water levels in the four tanks, denoted by $y 1, y 2, y 3$, and $y 4$, are the plant states, and the capacity of the tanks (i.e., $16 \mathrm{~cm}$ height) define the constraints of the system. The voltage of 


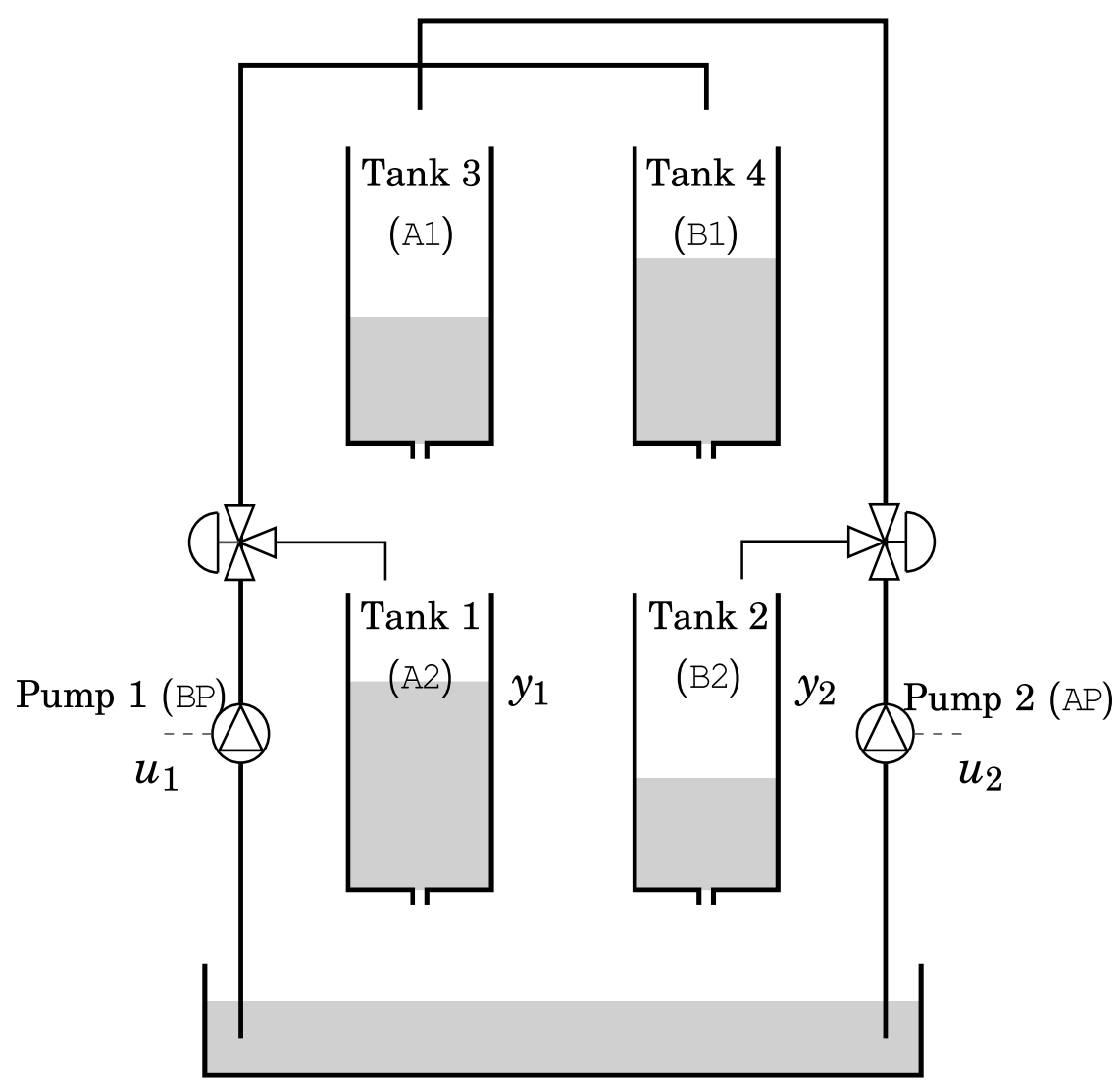

Figure 6.5: Schematic of the water tank system (Johansson, 2000).

the two pumps denoted by $u 1$ and $u 2$ are the control signals. The state-space model and the constraints can be written in the form of Equation 2.6 and Equation 2.7, respectively. Therefore, an MPC controller can be designed to control this water tank system where the cost function is in the standard format shown in Equation 2.8. Subsequently, we outsource the controller to the cloud and use verifiable computation to preserve the integrity of control input signals.

The sampling period for the MPC controller is set to $3 \mathrm{~s}$ and the prediction horizon is 30 steps. The controller (empowered by verifiable computation) is running on a Microsoft Azure cloud service. The Azure cloud uses an Intel Xeon 2.30GHz E5-2673 and 2 virtual CPUs (cost of 0.031 USD per hour). 

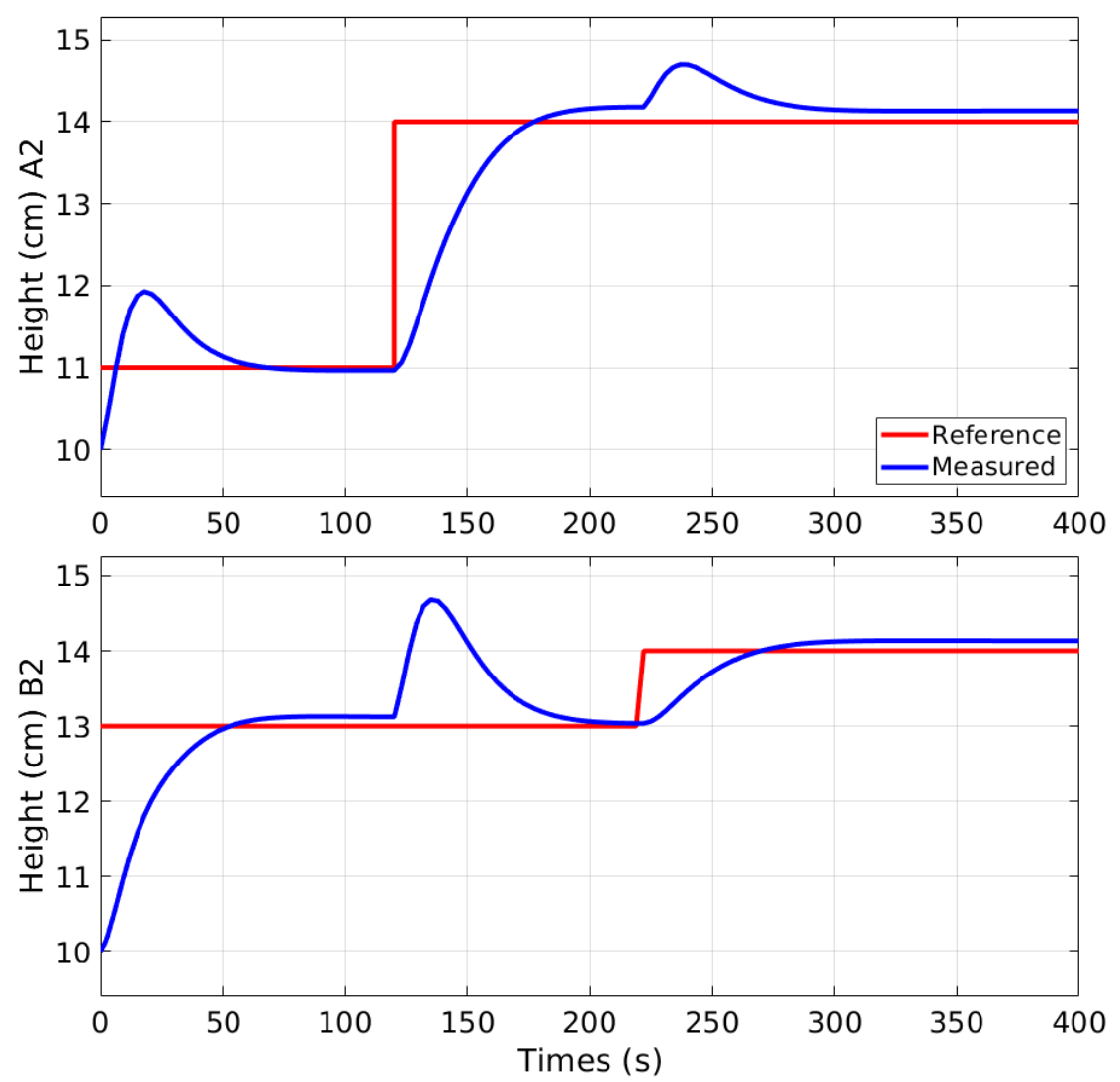

Figure 6.6: Water level in the two lower tanks. The red lines show the reference trajectory and the blue curves show the actual water level in tanks.

This setup provides us with an average round-trip delay (from the sensor to the actuator) of $0.992 \mathrm{~s}$ (less than the sampling period, i.e., 3s). Figure 6.4 separately specifies different parts of the round-trip delay. In this experiment, we first set the desired water level in the tanks $A 2$ and $B 2$ to $11 \mathrm{~cm}$ and $13 \mathrm{~cm}$, respectively. Then at time $120 \mathrm{~s}$ we change the desired water level in the tank $A 2$ to $14 \mathrm{~cm}$ and at time 220 we change the desired water level in the tank $B 2$ to $14 \mathrm{~cm}$. Note that the MPC constraints are set so that the water level in all the tanks does not reach $16 \mathrm{~cm}$. Figure 6.6 shows how the water levels converge to the reference values, while the MPC constraints are not violated. 
Please note that, in the case of the helicopter, which has more stringent timing requirements, we have to choose a more powerful processor in the cloud to be able to keep the system stable which also implies a higher cost (see Table 6.1).

In the two case studies, we have used simulated models of the two plants on a laptop. The laptop (which served as the client) was connected to the cloud service that was running the outsourced MPC controller. As opposed to this, in the next section, we are running an experiment with the physical water tank plant.

\subsection{Physical Water Tank Plant}

In this section, we describe an experiment performed with a real-life physical water tank plant in order to further investigate the applicability of verifiable computation to control applications. Figure 6.7 shows the schematic of the installation we use. The water tank system is connected via a serial port to a laptop that serves as the local controller. As opposed to the previous section however, there is no simulated plant model running on the laptop since we are using a physical water tank plant. The local controller uses a TCP connection to connect to the Microsoft Azure cloud servers. The Azure server we use in this experiment is running on an Intel Haswell 2.30 GHz E5-2673 v3 processor with 1 virtual CPU, which may be rented for 0.008 USD per hour.

We first use the KeyGen algorithm of the verifiable computation scheme to create the proof and the verification key. Then, we outsource the proof key to the cloud and store the verification key on the local controller.

The sampling period in this experiment is $3 \mathrm{~s}$ and the prediction horizon for the MPC controller is 30 steps. At each sampling period, the state of the system is sent to the local controller. The local controller then sends the data to the cloud via TCP connection. The cloud runs the MPC controller to calculate the output and then generates a proof using the proof key. The control output and the proof are then sent back to the local controller, where they are verified using the verification key. If the verification is successful, the control output is sent to the physical water tank system to be actuated. 


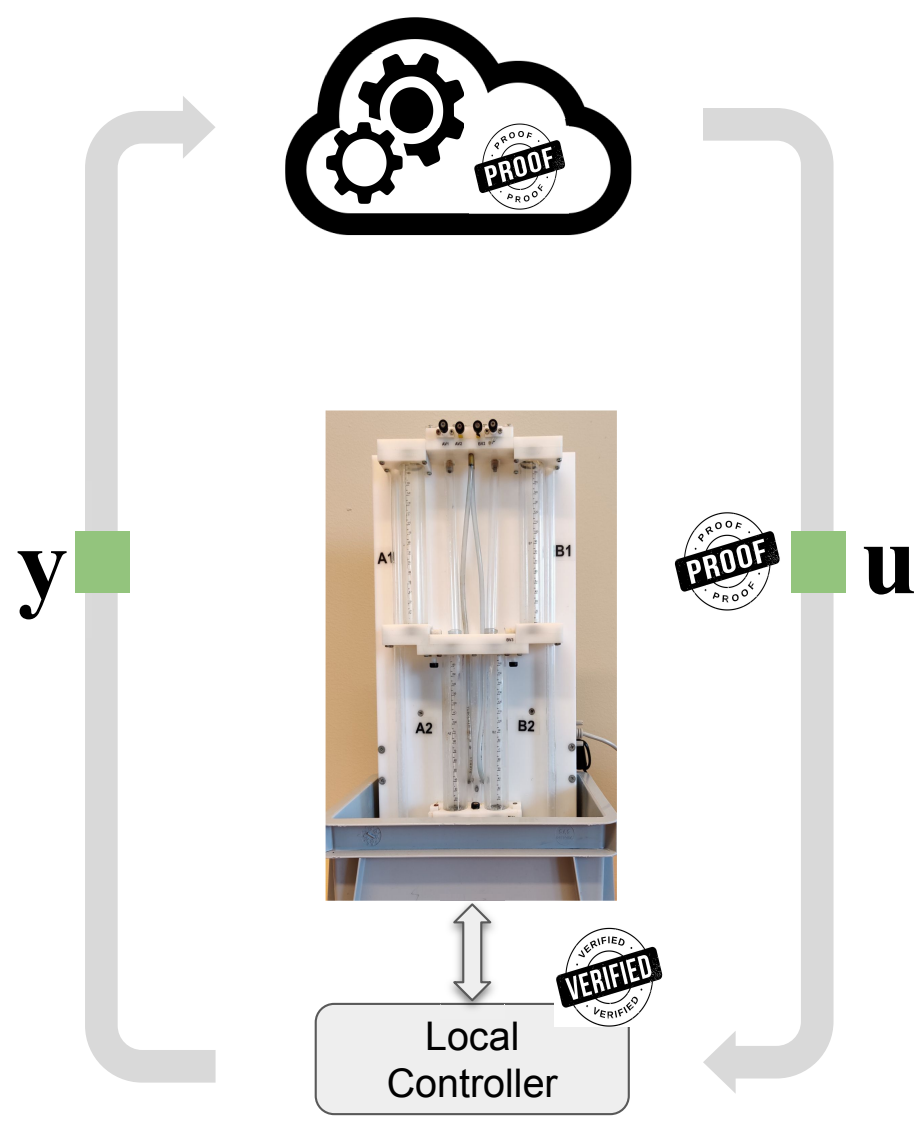

Figure 6.7: Water tank system controlled via cloud. In each sampling period the cloud calculates the output based on the state of the system and sends back the output and the proof to the local controller. 
During the experiment, the reference water levels in the two lower tanks are changed. A video showing the water tank system functioning is available in (Mahfouzi, 2021).

\subsection{Impact of Control Parameters and Cloud Server Performance}

In this section, we run several experiments to evaluate how controller parameters affect the delay imposed by verifiable computation. We also investigate the possibility of using more powerful cloud resources to reduce the computational delay of the calculation and proof generation in the cloud.

The computationally expensive part of the MPC controller is run in the Azure cloud. The Azure services enable us to run the MPC controller and proof generation on various processing units with different computational power.

For the experiments in this section, we consider the water tank system. However, the results regarding verification performance in the cloud and on the client are applicable to MPC controllers in general.

In the first experiment, we use a D-series Azure virtual machine that is running on an AMD EPYC 7452 processor with 2 virtual CPUs. Figure 6.8 shows how increasing the prediction horizon improves the control performance (decreases control cost). The control cost is calculated according to the standard quadratic cost formulated in Equation 2.3. The impact of increasing the prediction horizon on calculation and proof generation time in the cloud and verification time on the client is shown in Figure 6.9 and Figure 6.10. Increasing the prediction horizon leads to an increase in the computational load of the outsourced function, hence leading to longer prove and verify times in the cloud and on the client. For this example the control performance does not improve significantly after prediction horizon around $\boldsymbol{H}_{\boldsymbol{p}}=28$.

In the second experiment, the MPC parameters are fixed, $\boldsymbol{H}_{\boldsymbol{p}}=30$, and we run the experiment on 6 different cloud configurations as stated in Table 6.1. The second to last column in the table shows the time spent in the cloud to calculate the output and generate the proof. The reported times are averages that were calculated over 1000 repetitions of the experiment in the cloud (the 


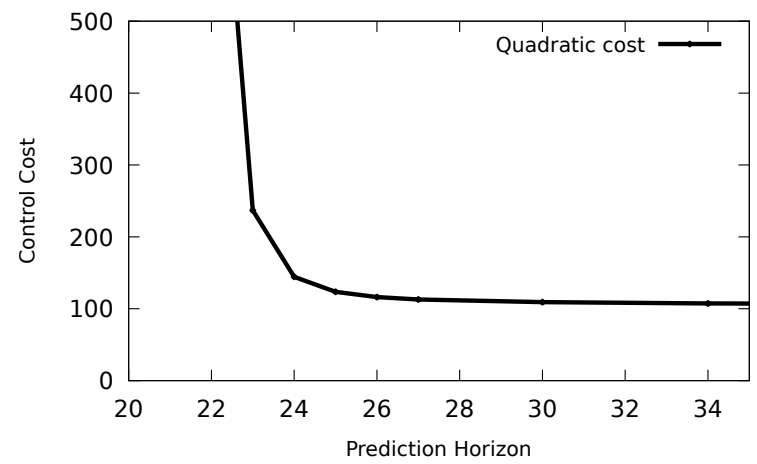

Figure 6.8: Quadratic control cost versus prediction horizon.

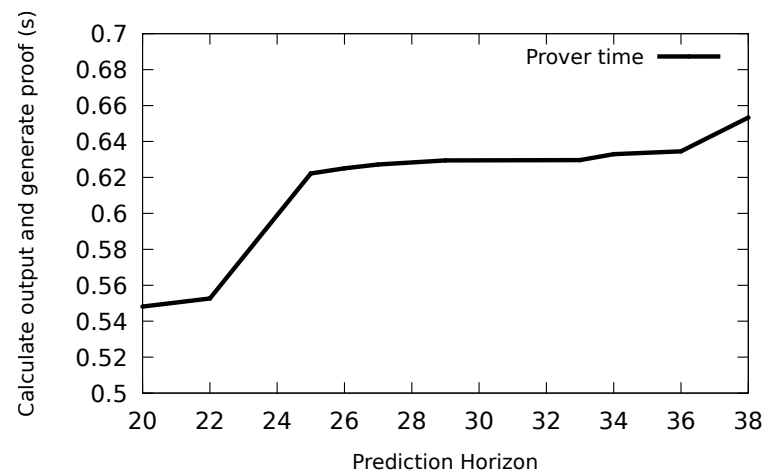

Figure 6.9: Time spent in the cloud to calculate output and generate proof for different prediction horizons.

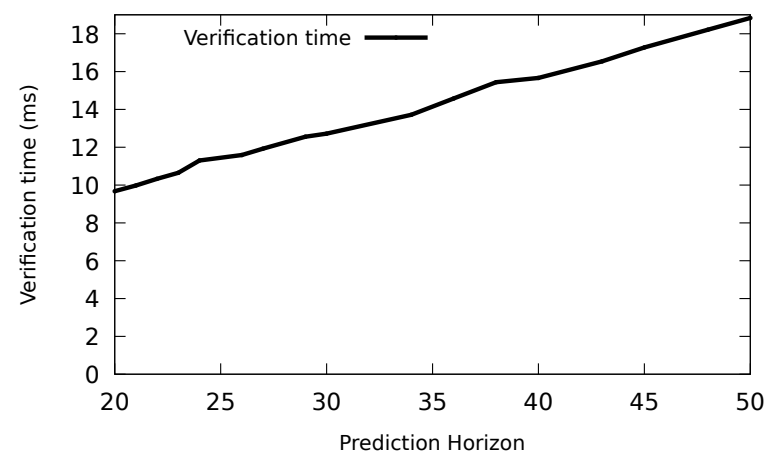

Figure 6.10: Time spent on the client to verify the proof for different prediction horizons. 
6. Secure Cloud-Based Control Using Verifiable Computation

Table 6.1: Time spent to calculate output and generate proof for various cloud configurations with different amounts of computational power.

\begin{tabular}{|c|c|c|c|c|}
\hline \multirow{2}{*}{ Serie } & CPU Model & vCPU's & $\begin{array}{c}\text { runtime } \\
\text { in the cloud (s) }\end{array}$ & $\begin{array}{c}\text { cost per hour } \\
\text { (in USD) }\end{array}$ \\
\hline \multirow{2}{*}{$\mathrm{A}$} & Intel Xeon & 2 & 2.45 & 0.028 \\
\cline { 3 - 5 } & $2.20 \mathrm{GHz}$ E5-2660 & 4 & 2.31 & 0.060 \\
\hline \multirow{2}{*}{$\mathrm{B}$} & Intel Xeon & 2 & 0.89 & 0.031 \\
\cline { 3 - 5 } & $2.30 \mathrm{GHz}$ E5-2673 & 4 & 0.83 & 0.062 \\
\hline \multirow{2}{*}{$\mathrm{D}$} & AMD EPYC & 2 & 0.63 & 0.036 \\
\cline { 3 - 5 } & 7452 & 4 & 0.60 & 0.073 \\
\hline
\end{tabular}

standard deviation is negligible). This experiment shows that by using more powerful cloud servers, one can reduce the time spent on calculating the output and generating the proof. Note that although the cost of renting a server with more virtual CPU cores is higher, the improvement in the performance of verifiable computation is insignificant due to the lack of parallelism in the Pequin implementation, hence the non-monotonic relation between cost and runtime in the cloud in Table 6.1.

We have shown, experimentally, that with a basic cloud configuration the round-trip time is kept below 1s (see Figure 6.4). This makes the scheme applicable for control applications with sampling periods of $1 \mathrm{~s}$ or more.

According to our experiments, the majority of the round-trip time is due to the time spent on generating the proof in the cloud (see the green boxes in Figure 6.4). We have shown experimentally that this delay can be reduced dramatically with more powerful cloud servers (see Table 6.1).

\subsection{Discussion}

In this chapter, we have experimentally demonstrated the applicability of verifiable computation on two control systems. The proposed solution is generalizable to any control system using MPC that solves the optimization problem in Equation 2.9. Our experiments (Figure 6.4) show that creating the proof in the cloud is responsible for the majority of the delay. We anticipate that 
this delay can be reduced by improving the efficiency of verifiable computation schemes in the cloud (Walfish and Blumberg, 2015). Moreover, the delay can be dramatically reduced by using more powerful cloud servers (see Table 6.1). By using the proposed solution, the following security measures are guaranteed:

1. If the cloud provider miscalculates or intentionally returns the wrong output, the local controller is able to verify and prove the misdeed.

2. The manipulation of data in the communication channel is also verifiable by the client.

In these two cases, the local controller discards the output from the cloud and will take further safety measures, e.g., by using a simple backup controller (Skarin et al., 2020), applying delay mitigation techniques (Mubeen et al., 2017), or raising an alarm.

3. The control algorithm that has been outsourced to the cloud remains secret from the local controller.

\subsection{Conclusion}

In this chapter, we have delivered, for the first time, a proof of concept for the applicability of verifiable computation in real-life control systems. Several experiments demonstrated secure cloud-based implementations of MPC controllers using verifiable computation. The experiments indicate that, when using a basic cloud computing server, the verifiable computation scheme is applicable for real-life control applications.

Moreover, research in the past few years in the field of verifiable computation has improved the performance of these schemes by an order of magnitude, especially on the verification side. Given the fact that this line of research is very active, further improvement in verifiable computation is expected. Hence, we anticipate that in the future it will be possible to apply verifiable computation schemes to control applications with even more rigorous timing constraints than the ones used in our experiments. 


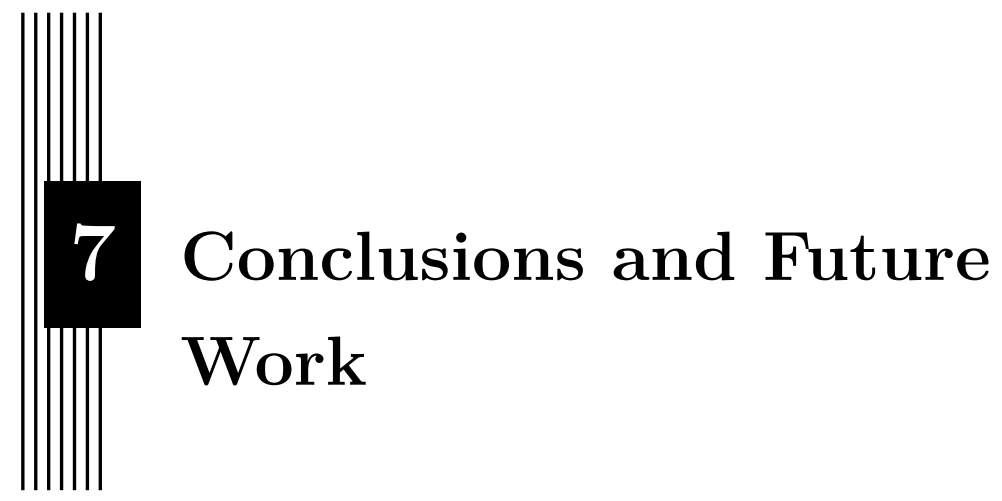

Cyber-security is fast becoming a key requirement in computer systems. In a wide range of IT systems, security is now recognized as a design time requirement, not a feature that is incorporated during deployment. In Chapter 1 we argued that conventional IT-based security measures are not sufficient in the case of CPSs due to the specific characteristics of these systems.

In this study, we focused on several security issues related to CPSs for control applications. We specifically aimed at the security threats posed to CPSs due to sharing of resources and the interplay between scheduling and stability. The proposed security measures demonstrate the necessity for integrating security into the design and synthesis of CPSs for control applications.

In the rest of this chapter, we conclude the thesis and discuss some interesting future research directions. 


\subsection{Conclusions}

In Chapter 3 we introduced an anomaly detection and mitigation mechanism for control systems (RQ1). We defined the notion of observable and controllable attacks and demonstrated the effectiveness of our intrusion detection and mitigation mechanism for this class of attacks. Our approach exploited the physical properties of the plant and used a machine learning algorithm to capture the normal behavior of the system. We also exploited the inherent properties of control tasks on a shared platform to provide an intrusion mitigation approach based on a feedback resource allocation mechanism. The key to this mitigation mechanism was the correlation between control performance and the amount of resources allocated to the control application. Note that while this correlation exists in many CPSs, it may not hold in the worst-case scenario in some cases, as shown in Chapter 4.

In Chapter 4, another timing property of control tasks on a shared platform was investigated. In that chapter, we demonstrated the inter-dependency of temporal properties and stability of tasks and the non-monotonicity of jitter with respect to scheduling parameters. We showed that, for jittersensitive control applications in certain circumstances, increasing the amount of allocated resources for a control application does not necessarily lead to better control performance. Moreover, we elaborated on how an attacker might exploit this complex timing behavior to destabilize a control application (RQ2).

In Chapter 5 we considered a CPS wherein an Ethernet TSN network was used as a shared communication channel for several control applications. First, we addressed the joint schedule-route synthesis problem for control applications communicating over a time-triggered Ethernet TSN network (RQ3a). We proposed a stability-aware routing and scheduling approach that accounted for the worst-case stability requirements of the control applications. Then, we considered the security threats of an intruder trying to sniff or modify timetriggered messages in the network. We proposed a security and stability-aware methodology that considers security measures in addition to the stability requirements and provides routes and schedules for messages in the Ethernet network. The goal was to maximize the security of control applications while guaranteeing the stability of control plants (RQ3b). 
Finally, in Chapter 6 we focused on the security challenges implied by outsourcing a control application to a cloud-based platform. We proposed the use of the verifiable computation technique to preserve the integrity of messages being transmitted to/from the cloud as well as the integrity of the computation done in the cloud. Although the practicality of verifiable computation for a general cloud application is expected in the future, its applicability has never been investigated for control applications. In Chapter 6 , we experimentally showed the applicability of this technique to real-life control applications (RQ4).

\subsection{Future Work}

The ideas proposed in this thesis can be used as a basis for further research. In this section, we outline some of these future research directions. Moreover, there are many experimental ideas to enrich the results achieved in each chapter that have been skipped due to the time and other delimitations of the Ph.D. studies but can further demonstrate the applicability of these approaches in the real life. We also mention these additional experimental investigations in this section.

In Chapter 3, we delivered an intrusion detection and mitigation mechanism for control applications that are using a shared resource. We proposed a mitigation mechanism using a resource management technique. Further research might explore more advanced mitigation mechanisms. One idea is the use of state estimation for cases in which the input from the physical plant is manipulated and intrusion is detected. In this case, the state estimator uses the model of the physical plant to estimate the internal state of the system. Since intrusion has been detected and the real state from the physical plant cannot be used, this estimated state could be utilized to control the physical plant. Enhancing state estimation techniques with the intrusion mitigation approach proposed in Chapter 3 could lead to a more robust control design that counteracts more powerful intrusions.

To enrich the research done in Chapter 3, further experiments to demonstrate the applicability of the intrusion detection and mitigation approach on a reallife example would be of great value. The real-life setting can be built con- 
sidering the shared platform with the two inverted pendulums that was used for the experiments in Chapter 3. Physical inverted pendulums developed for research and educational purposes, like the Quanser QUBE-Servo (Quanser Inc., 2019b), could be used for the two plants, and a microcontroller such as NI myRIO, Arduino or Raspberry Pi could be used as a shared processor. Running the same experiments we performed in Chapter 3 on a real-life example could provide more information about the applicability of the intrusion detection and mitigation approach. Moreover, intrusion by means of physical manipulation of the control system (i.e., an intruder trying to physically destabilize the plant) could also be investigated.

In Chapter 4 we introduced the Butterfly attack, a class of temporal attacks used to indirectly destabilize a physical plant. We used the JitterMargin toolbox to determine if a non-ideal timing scenario could lead to instability of a physical plant. A newer toolbox called JitterTime (Cervin et al., 2019) claims to provide much less pessimistic results than JitterMargin when the actual schedule is known. Further experiments using JitterTime instead of JitterMargin could lead to a better understanding of timing anomalies and could more closely link timing anomalies to the possibility of carrying out a Butterfly attack.

In a case study in Chapter 4 we demonstrated how a Butterfly attack could be used to destabilize or even hijack a drone. The experiment was done using a simulation of a quadcopter in Simulink MATLAB. The results suggest that the attack is applicable in cases where the navigation control task of the drone is jitter-sensitive. This underscores the need for further real-life experiments on real-life control systems.

Ethernet is fast becoming a key instrument in real-time communication in many networked CPSs, especially in the automotive and avionic industries. In Chapter 5 we investigated the message scheduling and routing problem in CPSs based on the time-triggered Ethernet TSN standards. The interaction of the safety critical, time-triggered Ethernet network with other networks in the CPSs, e.g., the infotainment system in a car, makes them vulnerable to intrusion attacks like the Butterfly attack which was introduced in Chapter 4. The discussion about the Butterfly attack in Chapter 4 was mostly for situations wherein the processor is used as a shared platform, but the definition of 
the Butterfly attack is not in any way restricted to this assumption. Therefore, this attack and other types of temporal attacks are typically applicable to Ethernet TSN networks. Mitigating temporal attacks by synthesizing a resilient schedule and route for time-triggered messages in the Ethernet TSN would be a fruitful area for further work. Synthesizing a resilient schedule and route can be done using the same design exploration approach (SMT formulation) proposed in Chapter 5, as well as for some additional constraints like route and schedule replication. Further research could also be conducted by using standardized protocols such as frame replication and elimination (IEEE 802.1CB), which are part of the Ethernet TSN standard (IEEE Standard, 2017), as a background theory and then formulating proper constraints to address the attack mitigation problem - similar to what Zhou et al. (Zhou et al., 2021a) recently proposed.

With expansion of distributed control over the network, controller tasks are being more and more migrated to the cloud or to fog networks. This makes the outsourced controllers vulnerable to security threats. In Chapter 6 we investigated the security issues related to the integrity of the data received by the local controller, ignoring the confidentiality and availability of the framework. We focused on an MPC controller being outsourced to the cloud. We also used a modern cryptographic technique called verifiable computation to preserve the integrity of data and computation results. In an outsourcing context, wherein an MPC controller is outsourced to the cloud, confidentiality of data transmitted to the cloud is also important (it might be desired that the cloud does work on encrypted data and is not aware of the actual data generated by the physical plant). This aspect is not addressed with verifiable computation and is orthogonal to it. One approach to addressing the confidentiality issue is to use a modern cryptographic technique called homomorphic encryption. Homomorphic encryption is a form of encryption that permits the cloud to perform computation on data without first decrypting it. The authors of (Alexandru et al., 2020) have recently proposed a protocol to achieve data privacy, i.e., the input and output to/from the cloud remain a secret that is known only to the local controller, and the cloud cannot obtain this private data. Their protocol is based on a projected gradient ascent on the Lagrange dual problem and exploits a partially homomorphic encryption technique. However, to reduce the computational complexity of the protocol 
some privacy guarantees are sacrificed in this method. On the other hand, fully homomorphic encryption techniques that guarantee the privacy of the data and their performance have been improved in the recent years. Many practical implementations of fully homomorphic encryption are being developed by pioneer research groups (IBM, 2021; Microsoft, 2021). An interesting idea for future work is to implement the MPC controller in a fully homomorphic framework and investigate the overhead of using this technique to preserve data privacy, and then compare the results with the current state-of-the art approaches.

In Chapter 6 we used Pequin, which is an implementation of a verifiable computation technique. We demonstrated that the overhead of running this protocol in the cloud in order to generate the proof is the main bottleneck of reducing the timing overhead. The verifiable computation protocol and Pequin are designed and implemented to permit general computation to be securely outsourced to the cloud. With certain knowledge about the underlying computation, the verifiable computation can be redesigned and implemented more efficiently for only that specific computation. 


\section{Bibliography}

Åkesson, Johan (2006). MPCtools 1.0 - Reference Manual. Technical Report TFRT-7613. Department of Automatic Control, Lund Institute of Technology, Lund University.

Alexandru, Andreea B, Konstantinos Gatsis, Yasser Shoukry, Sanjit A Seshia, Paulo Tabuada, and George J Pappas (2020). "Cloudbased quadratic optimization with partially homomorphic encryption". In: IEEE Transactions on Automatic Control 66.5, pp. 23572364.

Alexandru, Andreea B, Manfred Morari, and George J Pappas (2018). "Cloud-based MPC with encrypted data". In: 2018 IEEE Conference on Decision and Control (CDC). IEEE, pp. 5014-5019.

Amin, Saurabh, Alvaro A Cárdenas, and S Shankar Sastry (2009). "Safe and secure networked control systems under denial-of-service attacks". In: International Workshop on Hybrid Systems: Computation and Control. Springer, pp. 31-45.

Amin, Saurabh, Xavier Litrico, S Shankar Sastry, and Alexandre M Bayen (2010). "Stealthy deception attacks on water SCADA sys- 
tems". In: Proceedings of the 13th ACM international conference on Hybrid systems: computation and control. ACM, pp. 161-170.

Aminifar, Amir (2016). "Analysis, Design, and Optimization of Embedded Control Systems". PhD thesis. Linköping University, Software and Systems, p. 155. ISBN: 978-91-7685-826-4. DOI: 10.3384/diss. diva-124319.

Aminifar, Amir, Enrico Bini, Petru Eles, and Zebo Peng (2015a). "Analysis and design of real-time servers for control applications". In: IEEE Transactions on Computers 65.3, pp. 834-846.

Aminifar, Amir, Petru Eles, and Zebo Peng (2015b). "Jfair: A scheduling algorithm to stabilize control applications". In: 21st IEEE Real-Time and Embedded Technology and Applications Symposium. IEEE, pp. 63-72.

- (2017). "Optimization of message encryption for real-time applications in embedded systems". In: IEEE Transactions on Computers 67.5, pp. 748-754.

Aminifar, Amir, Petru Eles, Zebo Peng, and Anton Cervin (2013a). "Control-quality driven design of cyber-physical systems with robustness guarantees". In: 2013 Design, Automation 83 Test in Europe Conference \& Exhibition (DATE). IEEE, pp. 1093-1098.

- (2013b). "Stability-aware analysis and design of embedded control systems". In: 2013 Proceedings of the International Conference on Embedded Software (EMSOFT). IEEE, pp. 1-10.

Aminifar, Amir, Petru Eles, Zebo Peng, Anton Cervin, and Karl-Erik Årzén (2018). "Control-Quality Driven Design of Embedded Control Systems with Stability Guarantees". In: IEEE Design 83 Test 35.4, pp. 38-46.

Aminifar, Amir, Soheil Samii, Petru Eles, Zebo Peng, and Anton Cervin (2012). "Designing High-Quality Embedded Control Systems with Guaranteed Stability". In: Proceedings of the $33^{\text {th }}$ IEEE Real-Time Systems Symposium, pp. 283-292. 
Anand, Madhukar, Eric Cronin, Micah Sherr, Matt Blaze, Zachary Ives, and Insup Lee (2006). "Security challenges in next generation cyber physical systems". In: Beyond SCADA: Networked Embedded Control for Cyber Physical Systems 41.

Anderson, David P, Jeff Cobb, Eric Korpela, Matt Lebofsky, and Dan Werthimer (2002). "SETI@ home: an experiment in public-resource computing". In: Communications of the ACM 45.11, pp. 56-61.

Aoudi, Wissam, Mikel Iturbe, and Magnus Almgren (2018). "Truth will out: Departure-based process-level detection of stealthy attacks on control systems". In: Proceedings of the 2018 ACM SIGSAC Conference on Computer and Communications Security, pp. 817-831.

ArduPilot Team (2021). ArduPilot Project. http://ardupilot.org/. Accessed: 2021-07-27.

Årzén, K. E., A. Cervin, J. Eker, and L. Sha (2000). "An Introduction to Control and Scheduling Co-Design". In: Proceedings of the $39^{\text {th }}$ IEEE Conference on Decision and Control, pp. 4865-4870.

Årzén, Karl-Erik and Anton Cervin (2005). "Control and embedded computing: Survey of research directions". In: IFAC Proceedings Volumes 38.1, pp. 191-202.

Årzén, Karl-Erik, Per Skarin, William Tärneberg, and Maria Kihl (2018). "Control over the Edge Cloud-An MPC Example". In: 1st International Workshop on Trustworthy and Real-time Edge Computing for Cyber-Physical Systems.

Ashjaei, Mohammad, Mikael Sjödin, and Saad Mubeen (2021). "A novel frame preemption model in TSN networks". In: Journal of Systems Architecture 116, p. 102037.

Åström, K. J. and B. Wittenmark (1997). Computer-Controlled Systems. 3rd ed. Prentice Hall.

Avni, Guy, Shibashis Guha, and Guillermo Rodriguez-Navas (2016). "Synthesizing time-triggered schedules for switched networks with faulty links". In: Proceedings of the 13th International Conference on Embedded Software, pp. 1-10. 
Barreto, Carlos, Alvaro A Cárdenas, and Nicanor Quijano (2013). "Controllability of dynamical systems: Threat models and reactive security". In: International Conference on Decision and Game Theory for Security. Springer, pp. 45-64.

Barrett, Clark, Aaron Stump, Cesare Tinelli, et al. (2010). "The smtlib standard: Version 2.0". In: Proceedings of the 8th International Workshop on Satisfiability Modulo Theories. Vol. 13, p. 14.

Behnam, Moris and Damir Isovic (2007). "Real-Time Control and Scheduling Co-Design for Efficient Jitter Handling". In: Proceedings of the $13^{\text {th }}$ IEEE International Conference on Embedded and Real-Time Computing Systems and Applications, pp. 516-524.

Bello, Lucia Lo (2011). "The case for ethernet in automotive communications". In: ACM SIGBED Review 8.4, pp. 7-15.

Bello, Lucia Lo, Riccardo Mariani, Saad Mubeen, and Sergio Saponara (2018). "Recent advances and trends in on-board embedded and networked automotive systems". In: IEEE Transactions on Industrial Informatics 15.2, pp. 1038-1051.

Bianchin, Gianluca and Fabio Pasqualetti (2018). "Time-delay attacks in network systems". In: Cyber-Physical Systems Security. Springer, pp. 157-174.

Biere, Armin, Marijn Heule, and Hans van Maaren (2009). Handbook of satisfiability. Vol. 185. IOS press.

Bini, Enrico and Anton Cervin (2008). "Delay-aware period assignment in control systems". In: Proceedings of the $29^{\text {th }}$ Real-Time Systems Symposium. IEEE, pp. 291-300.

Biondi, Alessandro and Giorgio Buttazzo (2018). "Modeling and analysis of engine control tasks under dynamic priority scheduling". In: IEEE Transactions on Industrial Informatics 14.10, pp. 4407-4416.

Biondi, Alessandro, Marco Di Natale, and Giorgio Buttazzo (2017). "Response-time analysis of engine control applications under fixedpriority scheduling". In: IEEE Transactions on Computers 67.5, pp. 687-703. 
Bjørner, Nikolaj, Anh-Dung Phan, and Lars Fleckenstein (2015). " $\nu \mathrm{z}$ an optimizing SMT solver". In: International Conference on Tools and Algorithms for the Construction and Analysis of Systems. Springer, pp. 194-199.

Blumberg, Andrew J., Max Howald, Srinath Setty, Riad S. Wahby, and Michael Walfish (2021). Pepper: toward practical verifiable computation. https://www. pepper-project.org/. Accessed: 2021-07-27.

Bogdanov, Andrey, Dmitry Khovratovich, and Christian Rechberger (2011). "Biclique cryptanalysis of the full AES". In: International Conference on the Theory and Application of Cryptology and Information Security. Springer, pp. 344-371.

Borst, Johan, Bart Preneel, and Joos Vandewalle (1999). "Linear Cryptanalysis of RC5 and RC6". In: International Workshop on Fast Software Encryption. Springer, pp. 16-30.

Bouabdallah, Samir (2007). "Design and control of quadrotors with application to autonomous flying". PhD thesis. École Polytechnique Fédérale de Lausanne (EPFL), p. 155. DOI: 10. 5075/epfl-thesis3727.

Bouchenak, Sara, Gregory Chockler, Hana Chockler, Gabriela Gheorghe, Nuno Santos, and Alexander Shraer (2013). "Verifying cloud services: present and future". In: ACM SIGOPS operating systems review 47.2, pp. 6-19.

Bund, Tobias and Frank Slomka (2013). "A Delay Density Model for Networked Control Systems". In: Proceedings of the $21^{\text {st }}$ International Conference on Real-Time Networks and Systems, pp. 205212.

Burns, Alan and Robert I Davis (2018). "A survey of research into mixed criticality systems". In: ACM Computing Surveys (CSUR) 50.6, p. 82.

Cárdenas, Alvaro A, Saurabh Amin, and Shankar Sastry (2008a). "Secure control: Towards survivable cyber-physical systems". In: The 
28th International Conference on Distributed Computing Systems Workshops. IEEE, pp. 495-500.

Cárdenas, Alvaro A., Saurabh Amin, Zong-Syun Lin, Yu-Lun Huang, Chi-Yen Huang, and Shankar Sastry (2011). "Attacks Against Process Control Systems: Risk Assessment, Detection, and Response". In: Proceedings of the 6th ACM Symposium on Information, Computer and Communications Security, pp. 355-366.

Cárdenas, Alvaro A., Saurabh Amin, and Shankar Sastry (2008b). "Research Challenges for the Security of Control Systems". In: Proceedings of the 3rd Conference on Hot Topics in Security. USENIX Association.

Cervin, Anton (2003). "Integrated Control and Real-Time Scheduling". eng. PhD thesis. Lund University.

- (2012). "Stability and Worst-Case Performance Analysis of Sampled-Data Control Systems with Input and Output Jitter". In: Proceedings of the 2012 American Control Conference (ACC), pp. $1-10$.

Cervin, Anton and Johan Eker (2003). "The Control Server: A computational model for real-time control tasks". In: 15th Euromicro Conference on Real-Time Systems, 2003. Proceedings. IEEE, pp. 113120.

Cervin, Anton, Dan Henriksson, Bo Lincoln, Johan Eker, and Karl Erik Årzén (2003). "How Does Control Timing Affect Performance? Analysis and Simulation of Timing Using Jitterbug and TrueTime". In: IEEE Control Systems Magazine 23.3, pp. 16-30.

Cervin, Anton, Bo Lincoln, Johan Eker, Karl Erik Årzén, and Giorgio Buttazzo (2004). "The Jitter Margin and Its Application in the Design of Real-Time Control Systems". In: Proceedings of the $10^{\text {th }}$ International Conference on Real-Time and Embedded Computing Systems and Applications, pp. 1-10.

Cervin, Anton, Paolo Pazzaglia, Mohammadreza Barzegaran, and Rouhollah Mahfouzi (2019). "Using jittertime to analyze transient 
performance in adaptive and reconfigurable control systems". In: 24th IEEE International Conference on Emerging Technologies and Factory Automation (ETFA). IEEE, pp. 1025-1032.

Chakraborty, Samarjit, Mohammad Abdullah Al Faruque, Wanli Chang, Dip Goswami, Marilyn Wolf, and Qi Zhu (2016). "Automotive cyber-physical systems: A tutorial introduction". In: IEEE Design E Test 33.4, pp. 92-108.

Chakraborty, Samarjit, Simon Kunzli, and Lothar Thiele (2002). "Approximate schedulability analysis". In: 23rd IEEE Real-Time Systems Symposium. IEEE, pp. 159-168.

Chandola, Varun, Arindam Banerjee, and Vipin Kumar (2009). "Anomaly detection: A survey". In: ACM computing surveys (CSUR) 41.3, pp. 1-58.

Chang, Wanli, Dip Goswami, Samarjit Chakraborty, and Arne Hamann (2018). "OS-aware automotive controller design using non-uniform sampling". In: ACM Transactions on Cyber-Physical Systems 2.4, p. 26.

Cheon, Jung Hee, Kyoohyung Han, Seong-Min Hong, Hyoun Jin Kim, Junsoo Kim, Suseong Kim, Hosung Seo, Hyungbo Shim, and Yongsoo Song (2018). "Toward a secure drone system: Flying with realtime homomorphic authenticated encryption". In: IEEE access 6, pp. 24325-24339.

Cheung, Steven, Bruno Dutertre, Martin Fong, Ulf Lindqvist, Keith Skinner, and Alfonso Valdes (2007). "Using model-based intrusion detection for SCADA networks". In: Proceedings of the SCADA security scientific symposium. Vol. 46. Citeseer, pp. 1-12.

Contini, Scott, Ronald L. Rivest, M. J. B. Robshaw, and Yiqun Lisa Yin (1998). Security of the RC6 TM Block Cipher.

Cormen, Thomas H., Clifford Stein, Ronald L. Rivest, and Charles E. Leiserson (2001). Introduction to Algorithms. 2nd. McGraw-Hill Higher Education. ISBN: 0070131511. 
Costello, Craig, Cédric Fournet, Jon Howell, Markulf Kohlweiss, Benjamin Kreuter, Michael Naehrig, Bryan Parno, and Samee Zahur (2015). "Geppetto: Versatile verifiable computation". In: IEEE Symposium on Security and Privacy. IEEE, pp. 253-270.

Craciunas, Silviu S, Ramon Serna Oliver, Martin Chmelik, and Wilfried Steiner (2016). "Scheduling real-time communication in IEEE 802.1 Qbv time sensitive networks". In: Proceedings of the 24th International Conference on Real-Time Networks and Systems, pp. 183192.

Darup, Moritz Schulze, Adrian Redder, Iman Shames, Farhad Farokhi, and Daniel Quevedo (2017). "Towards encrypted MPC for linear constrained systems". In: IEEE Control Systems Letters 2.2, pp. 195-200.

Davidson, Drew, Hao Wu, Rob Jellinek, Vikas Singh, and Thomas Ristenpart (2016). "Controlling UAVs with sensor input spoofing attacks". In: 10th USENIX Workshop on Offensive Technologies (WOOT).

De Moura, Leonardo and Nikolaj Bjørner (2008). "Z3: An efficient SMT solver". In: Tools and Algorithms for the Construction and Analysis of Systems.

Deligne, Eddy (2012). "ARDrone corruption". In: Journal in Computer Virology 8.1-2, pp. 15-27.

Deng, Peng, Qi Zhu, Abhijit Davare, Anastasios Mourikis, Xue Liu, and Marco Di Natale (2016). "An efficient control-driven period optimization algorithm for distributed real-time systems". In: IEEE Transactions on Computers 65.12, pp. 3552-3566.

Denning, Dorothy E (1987). "An intrusion-detection model". In: IEEE Transactions on software engineering 2, pp. 222-232.

Dhruva, Neil, Pratyush Kumar, Georgia Giannopoulou, and Lothar Thiele (2014). "Computing a Language-based Guarantee for Timing Properties of Cyber-physical Systems". In: Proceedings of the $17^{\text {th }}$ 
Conference for Design, Automation and Test in Europe (DATE). Dresden, Germany.

Di Natale, Marco and Alberto L. Sangiovanni Vincentelli (2010). "Moving from federated to integrated architectures in automotive: The role of standards, methods and tools". In: Proceedings of the IEEE 98.4, pp. 603-620.

Drozhzhin, Alex (2015). "Black Hat USA 2015: The full story of how that Jeep was hacked". In: Kaspersky Lab Daily 6.

Dworkin, Morris (2003). "Block Cipher Modes of Operation: The CCM Mode For Authentication and Confidentiality". In: National Institute of Standards $\&$ Technology special publication 800, p. 38C.

Dworkin, Morris J (2007). Recommendation for block cipher modes of operation: Galois/counter mode (gcm) and gmac. Vol. 800. National Institute of Standards \& Technology special publication, p. 38D.

Eltze, Jens (1997). "Double-CAN controller as bridge for different CAN networks". In: Proceedings of the 4th International CAN Conference.

Erdős, Paul, Alfréd Rényi, et al. (1960). "On the evolution of random graphs". In: Publ. Math. Inst. Hung. Acad. Sci 5.1, pp. 17-60.

Esen, Hasan, Masakazu Adachi, Daniele Bernardini, Alberto Bemporad, Dominik Rost, and Jens Knodel (2015). "Control as a service (CaaS) cloud-based software architecture for automotive control applications". In: Proceedings of the Second International Workshop on the Swarm at the Edge of the Cloud, pp. 13-18.

Farokhi, Farhad, Iman Shames, and Nathan Batterham (2017). "Secure and private control using semi-homomorphic encryption". In: Control Engineering Practice 67, pp. 13-20.

Farraj, Abdallah K, Eman M Hammad, Ashraf Al Daoud, and Deepa Kundur (2014). "A game-theoretic control approach to mitigate cyber switching attacks in smart grid systems". In: IEEE International Conference on Smart Grid Communications (SmartGridComm). IEEE, pp. 958-963. 
Farwell, James P and Rafal Rohozinski (2011). "Stuxnet and the future of cyber war". In: Survival 53.1, pp. 23-40.

Fawzi, Hamza, Paulo Tabuada, and Suhas Diggavi (2011). "Secure state-estimation for dynamical systems under active adversaries". In: 49th annual allerton conference on communication, control, and computing (allerton). IEEE, pp. 337-344.

- (2012). "Security for control systems under sensor and actuator attacks". In: IEEE 51st IEEE Conference on Decision and Control $(C D C)$. IEEE, pp. 3412-3417.

- (2014). "Secure estimation and control for cyber-physical systems under adversarial attacks". In: IEEE Transactions on Automatic control 59.6, pp. 1454-1467.

Fiore, Dario, Anca Nitulescu, and David Pointcheval (2020). "Boosting verifiable computation on encrypted data". In: IACR International Conference on Public-Key Cryptography. Springer, pp. 124-154.

Fiore, Gabriella, Young Hwan Chang, Qie Hu, Maria Domenica Di Benedetto, and Claire J Tomlin (2017). "Secure state estimation for cyber physical systems with sparse malicious packet drops". In: 2017 American Control Conference (ACC). IEEE, pp. 1898-1903.

Fontanelli, Daniel, Luigi Palopoli, and Luca Abeni (2013a). "The Continuous Stream Model of Computation for Real-Time Control". In: Proceedings of the $34^{\text {th }}$ IEEE Real-Time Systems Symposium.

Fontanelli, Daniele, Luca Greco, and Luigi Palopoli (2013b). "Soft realtime scheduling for embedded control systems". In: Automatica 49.8, pp. 2330-2338.

Fontantelli, Daniele, Luigi Palopoli, and Luca Greco (2013). "Optimal CPU allocation to a set of control tasks with soft real-time execution constraints". In: Proceedings of the $16^{\text {th }}$ international conference on Hybrid systems: computation and control, pp. 233-242.

Franklin, Gene F, J David Powell, Abbas Emami-Naeini, and J David Powell (2002). Feedback control of dynamic systems. Vol. 4. Prentice hall Upper Saddle River, NJ. 
$\mathrm{Fu}$, Kevin and Wenyuan $\mathrm{Xu}$ (2018). "Risks of trusting the physics of sensors". In: Communications of the ACM 61.2, pp. 20-23.

Gaid, ME Mongi Ben, Arben Cela, and Yskandar Hamam (2006). "Optimal integrated control and scheduling of networked control systems with communication constraints: Application to a car suspension system". In: IEEE Transactions on Control Systems Technology 14.4, pp. 776-787.

Garcia-Teodoro, Pedro, Jesus Diaz-Verdejo, Gabriel Maciá-Fernández, and Enrique Vázquez (2009). "Anomaly-based network intrusion detection: Techniques, systems and challenges". In: computers 8 security 28.1-2, pp. 18-28.

Gavriluţ, Voica and Paul Pop (2016). "Traffic class assignment for mixed-criticality frames in TTEthernet". In: ACM Sigbed Review 13.4, pp. 31-36.

Ghafouri, Amin, Waseem Abbas, Aron Laszka, Yevgeniy Vorobeychik, and Xenofon Koutsoukos (2016). "Optimal thresholds for anomalybased intrusion detection in dynamical environments". In: International Conference on Decision and Game Theory for Security. Springer, pp. 415-434.

Ghafouri, Amin, Yevgeniy Vorobeychik, and Xenofon D. Koutsoukos (2018). "Adversarial Regression for Detecting Attacks in CyberPhysical Systems". In: CoRR abs/1804.11022. arXiv: 1804.11022.

Giraldo, Jairo, David Urbina, Alvaro Cardenas, Junia Valente, Mustafa Faisal, Justin Ruths, Nils Ole Tippenhauer, Henrik Sandberg, and Richard Candell (2018). "A survey of physics-based attack detection in cyber-physical systems". In: ACM Computing Surveys (CSUR) 51.4, pp. 1-36.

Givehchi, Omid, Jahanzaib Imtiaz, Henning Trsek, and Juergen Jasperneite (2014). "Control-as-a-service from the cloud: A case study for using virtualized PLCs". In: 10th IEEE Workshop on Factory Communication Systems (WFCS). IEEE, pp. 1-4. 
Givehchi, Omid, Henning Trsek, and Juergen Jasperneite (2013). "Cloud computing for industrial automation systems-A comprehensive overview". In: 18th Conference on Emerging Technologies E Factory Automation (ETFA). IEEE, pp. 1-4.

Goldreich, Oded (2008). Probabilistic proof systems: A primer. Now Publishers Inc.

Goswami, Dip, Martin Lukasiewycz, Reinhard Schneider, and Samarjit Chakraborty (2012). "Time-triggered implementations of mixedcriticality automotive software". In: Design, Automation \& Test in Europe Conference $\mathcal{E}$ Exhibition (DATE). IEEE, pp. 1227-1232.

Gupta, Abhishek, Cédric Langbort, and Tamer Başar (2010). "Optimal control in the presence of an intelligent jammer with limited actions". In: 49th IEEE Conference on Decision and Control (CDC). IEEE, pp. 1096-1101.

Hegazy, Tamir and Mohamed Hefeeda (2014). "Industrial automation as a cloud service". In: IEEE Transactions on Parallel and Distributed Systems 26.10, pp. 2750-2763.

Hespanha, Joao P and A Stephen Morse (2002). "Switching between stabilizing controllers". In: Automatica 38.11, pp. 1905-1917.

Highnam, Kate, Kevin Angstadt, Kevin Leach, Westley Weimer, Aaron Paulos, and Patrick Hurley (2016). "An uncrewed aerial vehicle attack scenario and trustworthy repair architecture". In: 46th Annual IEEE/IFIP International Conference on Dependable Systems and Networks Workshop (DSN-W). IEEE, pp. 222-225.

Hoppe, Tobias, Stefan Kiltz, and Jana Dittmann (2008). "Security threats to automotive CAN networks-practical examples and selected short-term countermeasures". In: International Conference on Computer Safety, Reliability, and Security. Springer, pp. 235248.

Howald, Max and et al. (2019). Pequin: An end-to-end toolchain for verifiable computation, SNARKs, and probabilistic proofs. https: //github.com/pepper-project/pequin. 
Hwang, David D, Patrick Schaumont, Kris Tiri, and Ingrid Verbauwhede (2006). "Securing embedded systems". In: IEEE Computer Architecture Letters 4.02, pp. 40-49.

IBM (2021). IBM Fully Homomorphic Encryption Toolkit. https:// github.com/IBM/fhe-toolkit-linux. Accessed: 2021-09-16.

IEEE Standard (2015). IEEE Standard for Local and Metropolitan Area Networks - Bridges and Bridged Networks Amendment 25 : Enhancements for Scheduled Traffic, IEEE Std. 802.1Qbv-2015. DOI: 10.1109/IEEESTD . 2016.7440741.

- (2016). "IEEE Standard for Local and metropolitan area networks - Bridges and Bridged Networks - Amendment 26: Frame Preemption". In: IEEE Std 802.1Qbu-2016 (Amendment to IEEE Std 802.1Q-2014), pp. 1-52. DOI: 10.1109/IEEESTD. 2016.7553415.

- (2017). "IEEE Standard for Local and metropolitan area networksFrame Replication and Elimination for Reliability". In: IEEE Std 802.1CB-2017, pp. 1-102. DOI: 10.1109/IEEESTD.2017.8091139.

Inaltekin, Hazer, Maria Gorlatova, and Mung Chiang (2018). "Virtualized control over fog: Interplay between reliability and latency". In: IEEE Internet of Things Journal 5.6, pp. 5030-5045.

Ivanov, Radoslav, Miroslav Pajic, and Insup Lee (2014). "Attackresilient Sensor Fusion". In: Proceedings of the Conference on Design, Automation 83 Test in Europe (DATE), 54:1-54:6.

Izosimov, Viacheslav, Paul Pop, Petru Eles, and Zebo Peng (2012). "Scheduling and optimization of fault-tolerant embedded systems with transparency/performance trade-offs". In: ACM Transactions on Embedded Computing Systems (TECS) 11.3, pp. 1-35.

Jasmin, M and S Beulah Hemalatha (2017). "Security for industrial communication system using encryption/decryption modules". In: International Journal of Pure and Applied Mathematics 116.15, pp. 563-568.

Jiang, Jin (2005). "Fault-tolerant control systems-an introductory overview". In: Acta Automatica Sinica 31.1, pp. 161-174. 
Jiang, Ke, Petru Eles, and Zebo Peng (2011). "Optimization of message encryption for distributed embedded systems with real-time constraints". In: 14th IEEE International Symposium on Design and Diagnostics of Electronic Circuits and Systems. IEEE, pp. 243-248.

- (2012). "Co-design techniques for distributed real-time embedded systems with communication security constraints". In: Design, Automation $\&$ Test in Europe Conference $\&$ Exhibition (DATE). IEEE, pp. 947-952.

- (2013). "Optimization of secure embedded systems with dynamic task sets". In: 2013 Design, Automation 83 Test in Europe Conference $\mathcal{E}$ Exhibition (DATE). IEEE, pp. 1765-1770.

Johansson, Karl Henrik (2000). "The quadruple-tank process: A multivariable laboratory process with an adjustable zero". In: IEEE Transactions on control systems technology 8.3, pp. 456-465.

Jovanov, Ilija and Miroslav Pajic (2019). "Relaxing integrity requirements for attack-resilient cyber-physical systems". In: IEEE Transactions on Automatic Control 64.12, pp. 4843-4858.

Jutla, Charanjit S (2001). "Encryption modes with almost free message integrity". In: International Conference on the Theory and Applications of Cryptographic Techniques. Springer, pp. 529-544.

Kang, Kyoung-Don and Sang H Son (2006). "Systematic security and timeliness tradeoffs in real-time embedded systems". In: 12th IEEE International Conference on Embedded and Real-Time Computing Systems and Applications (RTCSA'06). IEEE, pp. 183-189.

Karray, Khaled, Jean-Luc Danger, Sylvain Guilley, and M Abdelaziz Elaabid (2018). "Identifier Randomization: An Efficient Protection Against CAN-Bus Attacks". In: Cyber-Physical Systems Security. Springer, pp. 219-254.

Käsper, Emilia and Peter Schwabe (2009). "Faster and timing-attack resistant AES-GCM". In: International Workshop on Cryptographic Hardware and Embedded Systems. Springer, pp. 1-17. 
Katz, Jonathan and Yehuda Lindell (2014). Introduction to modern cryptography. CRC press.

Kerns, Andrew J, Daniel P Shepard, Jahshan A Bhatti, and Todd E Humphreys (2014). "Unmanned aircraft capture and control via GPS spoofing". In: Journal of Field Robotics 31.4, pp. 617-636.

Khalid, Faiq, Semeen Rehman, and Muhammad Shafique (2020). "Overview of security for smart cyber-physical systems". In: $S e-$ curity of Cyber-Physical Systems. Springer, pp. 5-24.

Kim, Junsoo, Chanhwa Lee, Hyungbo Shim, Jung Hee Cheon, Andrey Kim, Miran Kim, and Yongsoo Song (2016). "Encrypting controller using fully homomorphic encryption for security of cyber-physical systems". In: IFAC-PapersOnLine 49.22, pp. 175-180.

Knudsen, Lars R and Willi Meier (2000). "Correlations in RC6 with a reduced number of rounds". In: International Workshop on Fast Software Encryption. Springer, pp. 94-108.

Kocher, Paul, Ruby Lee, Gary McGraw, and Anand Raghunathan (2004). "Security as a new dimension in embedded system design". In: Proceedings of the 41st annual Design Automation Conference, pp. $753-760$.

Kogiso, Kiminao and Takahiro Fujita (2015). "Cyber-security enhancement of networked control systems using homomorphic encryption". In: 54th IEEE Conference on Decision and Control (CDC). IEEE, pp. 6836-6843.

Koscher, Karl, Stefan Savage, Franziska Roesner, Shwetak Patel, Tadayoshi Kohno, Alexei Czeskis, Damon McCoy, Brian Kantor, Danny Anderson, Hovav Shacham, et al. (2010). "Experimental security analysis of a modern automobile". In: IEEE Symposium on Security and Privacy. IEEE Computer Society, pp. 447-462.

Kumar, Pratyush, Dip Goswami, Samarjit Chakraborty, Anuradha Annaswamy, Kai Lampka, and Lothar Thiele (2012). "A hybrid approach to cyber-physical systems verification". In: DAC Design Automation Conference 2012. IEEE, pp. 688-696. 
Kwon, Cheolhyeon, Weiyi Liu, and Inseok Hwang (2013). "Security analysis for cyber-physical systems against stealthy deception attacks". In: American control conference. IEEE, pp. 3344-3349.

Langner, Ralph (2011). "Stuxnet: Dissecting a cyberwarfare weapon". In: IEEE Security 83 Privacy 9.3, pp. 49-51.

Lee, Edward A (2006). "Cyber-physical systems-are computing foundations adequate". In: Position paper for NSF workshop on cyberphysical systems: research motivation, techniques and roadmap. Vol. 2. Citeseer, pp. 1-9.

- (2008). "Cyber physical systems: Design challenges". In: 11th IEEE international symposium on object and component-oriented realtime distributed computing (ISORC). IEEE, pp. 363-369.

Lemke, Kerstin, Christof Paar, and Marko Wolf (2006). Embedded security in cars. Springer.

Lemmon, Michael, Thidapat Chantem, Xiaobo Sharon $\mathrm{Hu}$, and Matthew Zyskowski (2007). "On Self-triggered Full-information Hinfinity Controllers". In: Proceedings of the 10th International Conference on Hybrid Systems: Computation and Control, pp. 371-384.

Lenstra, Arjen K (2004). Key Length. Contribution to The Handbook of Information Security.

Lesi, Vuk, Ilija Jovanov, and Miroslav Pajic (2017). "Security-aware scheduling of embedded control tasks". In: ACM Transactions on Embedded Computing Systems (TECS) 16.5s, pp. 1-21.

Li, Yi, Aws Albarghouthi, Zachary Kincaid, Arie Gurfinkel, and Marsha Chechik (2014). "Symbolic optimization with SMT solvers". In: ACM SIGPLAN Notices 49.1, pp. 607-618.

Lin, Man, Li Xu, Laurence T Yang, Xiao Qin, Nenggan Zheng, Zhaohui Wu, and Meikang Qiu (2009). "Static security optimization for realtime systems". In: IEEE Transactions on Industrial Informatics 5.1, pp. $22-37$.

Lincoln, Bo and Anton Cervin (2002). "Jitterbug: A tool for analysis of real-time control performance". In: Proceedings of the 41st IEEE 
Conference on Decision and Control, 2002. Vol. 2. IEEE, pp. 13191324.

Lindgren, Georg (2012). Stationary stochastic processes: theory and applications. CRC Press.

Lindsay, Jon R (2013). "Stuxnet and the limits of cyber warfare". In: Security Studies 22.3, pp. 365-404.

Liu, Chung Laung and James W Layland (1973). "Scheduling algorithms for multiprogramming in a hard-real-time environment". In: Journal of the ACM (JACM) 20.1, pp. 46-61.

Liu, Shichao, Peter X Liu, and Abdulmotaleb El Saddik (2014). "A stochastic game approach to the security issue of networked control systems under jamming attacks". In: Journal of the Franklin Institute 351.9, pp. 4570-4583.

Liu, Ting, Yanan Sun, Yang Liu, Yuhong Gui, Yucheng Zhao, Dai Wang, and Chao Shen (2015). "Abnormal traffic-indexed state estimation: A cyber-physical fusion approach for smart grid attack detection". In: Future Generation Computer Systems 49, pp. 94103.

Liu, Yao, Peng Ning, and Michael K Reiter (2011). "False data injection attacks against state estimation in electric power grids". In: ACM Transactions on Information and System Security (TISSEC) 14.1, p. 13.

Lou, Xin, Cuong Tran, Rui Tan, David KY Yau, and Zbigniew T Kalbarczyk (2019). "Assessing and mitigating impact of time delay attack: a case study for power grid frequency control". In: Proceedings of the 10th ACM/IEEE International Conference on CyberPhysical Systems. ACM, pp. 207-216.

Lun, Yuriy Zacchia, Alessandro D'Innocenzo, Francesco Smarra, Ivano Malavolta, and Maria Domenica Di Benedetto (2019). "State of the art of cyber-physical systems security: An automatic control perspective". In: Journal of Systems and Software 149, pp. 174216. 
Luo, Yuan, Ya Xiao, Long Cheng, Guojun Peng, and Danfeng Yao (2021). "Deep learning-based anomaly detection in cyber-physical systems: Progress and opportunities". In: ACM Computing Surveys (CSUR) 54.5, pp. 1-36.

Maciejowski, Jan Marian (2002). Predictive control: with constraints. Pearson education.

Mahfouzi, Rouhollah (2021). Watertank system in function. https: //youtu. be/URUoVBOxmmw?vq=hd1080.

Mahfouzi, Rouhollah, Amir Aminifar, Petru Eles, Zebo Peng, and Mattias Villani (2016). "Intrusion-damage assessment and mitigation in cyber-physical systems for control applications". In: Proceedings of the 24th International Conference on Real-Time Networks and Systems. ACM, pp. 141-150.

Mahfouzi, Rouhollah, Amir Aminifar, Soheil Samii, Petru Eles, and Zebo Peng (2019a). "Security-aware routing and scheduling for control applications on Ethernet TSN networks". In: ACM Transactions on Design Automation of Electronic Systems (TODAES) 25.1, pp. $1-26$.

- (2021). "Secure Cloud Control Using Verifiable Computation". In: IEEE International Conference on Omni-layer Intelligent systems (COINS). IEEE, pp. 93-106.

Mahfouzi, Rouhollah, Amir Aminifar, Soheil Samii, Mathias Payer, Petru Eles, and Zebo Peng (2019b). "Butterfly Attack: Adversarial Manipulation of Temporal Properties of Cyber-Physical Systems". In: 2019 IEEE Real-Time Systems Symposium (RTSS). IEEE, pp. 93-106.

Mahfouzi, Rouhollah, Amir Aminifar, Soheil Samii, Ahmed Rezine, Petru Eles, and Zebo Peng (2018). "Stability-aware integrated routing and scheduling for control applications in Ethernet networks". In: 2018 Design, Automation $\mathcal{E}$ Test in Europe Conference 83 Exhibition (DATE). IEEE, pp. 682-687. 
- (2020). "Breaking Silos to Guarantee Control Stability with Communication over Ethernet TSN". In: IEEE Design 83 Test.

Mancuso, Giulio, Enrico Bini, and Gabriele Pannocchia (2011). "Optimal computational resource allocation for control task under fixed priority scheduling". In: IFAC Proceedings Volumes 44.1, pp. 1259912604.

Mancuso, Giulio M, Enrico Bini, and Gabriele Pannocchia (2014). "Optimal priority assignment to control tasks". In: ACM Transactions on Embedded Computing Systems (TECS) 13.5s, pp. 1-17.

Marchetti, Mirco and Dario Stabili (2017). "Anomaly detection of CAN bus messages through analysis of ID sequences". In: IEEE Intelligent Vehicles Symposium (IV). IEEE, pp. 1577-1583.

Maul, Mario, Gerhard Becker, and Ulrich Bernhard (2018). "Serviceoriented EE zone architecture key elements for new market segments". In: ATZelektronik worldwide 13.1, pp. 36-41.

McAfee LLC (2019). What Is Stuxnet? https://www . mcafee.com/ enterprise/en-us/security-awareness/ransomware/what-isstuxnet.html. Accessed: 2021-06-20.

Messner, Bill and Dawn Tilbury (2017a). Control Tutorials for MAT$L A B$ and Simulink - Inverted Pendulum: System Modeling. https: / / ctms . engin . umich . edu / CTMS / index . php ? example = InvertedPendulum\&section=SystemModeling. Accessed: 2021-0727.

- (2017b). Control Tutorials for MATLAB and Simulink - Motor Speed: System Modeling. http://ctms . engin . umich. edu/CTMS / index $\cdot$ php? example=MotorSpeed\&section=SystemModeling. Accessed: 2021-07-27.

Miao, Fei, Quanyan Zhu, Miroslav Pajic, and George J Pappas (2016). "Coding schemes for securing cyber-physical systems against stealthy data injection attacks". In: IEEE Transactions on Control of Network Systems 4.1, pp. 106-117. 
Michalewicz, Zbigniew (1996). Genetic Algorithms + Data Structures = Evolution Programs (3rd Ed.) Berlin, Heidelberg: Springer-Verlag. ISBN: 3-540-60676-9.

Microsoft (2021). Microsoft SEAL. https://www. microsoft.com/enus/research/project/microsoft-seal/. Accessed: 2021-09-16.

Miller, Charlie and Chris Valasek (2015). "Remote exploitation of an unaltered passenger vehicle". In: Black Hat USA 2015.S 91.

Mo, Yilin, Tiffany Hyun-Jin Kim, Kenneth Brancik, Dona Dickinson, Heejo Lee, Adrian Perrig, and Bruno Sinopoli (2011). "Cyberphysical security of a smart grid infrastructure". In: Proceedings of the IEEE 100.1, pp. 195-209.

Mo, Yilin and Bruno Sinopoli (2014). "Secure estimation in the presence of integrity attacks". In: IEEE Transactions on Automatic Control 60.4, pp. 1145-1151.

Mohaqeqi, Morteza, Mitra Nasri, Yang Xu, Anton Cervin, and KarlErik Årzén (2018). "Optimal harmonic period assignment: complexity results and approximation algorithms". In: Real-Time Systems 54.4, pp. 830-860.

Montgomery, Douglas C, George C Runger, and Norma F Hubele (2009). Engineering statistics. John Wiley \& Sons.

Moon, Jun and Tamer Başar (2015). "Minimax control over unreliable communication channels". In: Automatica 59, pp. 182-193.

Mubeen, Saad, Pavlos Nikolaidis, Alma Didic, Hongyu Pei-Breivold, Kristian Sandström, and Moris Behnam (2017). "Delay mitigation in offloaded cloud controllers in industrial IoT". In: IEEE Access 5, pp. 4418-4430.

Mueller, Frank (2006). "Challenges for cyber-physical systems: Security, timing analysis and soft error protection". In: High-Confidence Software Platforms for Cyber-Physical Systems (HCSP-CPS) Workshop. Vol. 6.

Naghshtabrizi, Payam and Joao P Hespanha (2009). "Analysis of distributed control systems with shared communication and compu- 
tation resources". In: 2009 American Control Conference. IEEE, pp. 3384-3389.

Nechvatal, James, Elaine Barker, Lawrence Bassham, William Burr, Morris Dworkin, James Foti, and Edward Roback (2001). "Report on the development of the Advanced Encryption Standard (AES)". In: Journal of Research of the National Institute of Standards and Technology 106.3, p. 511.

Nghiem, Truong, George J. Pappas, Rajeev Alur, and Antoine Girard (2006). "Time-triggered implementations of dynamic controllers". In: Proceedings of the $6^{\text {th }} A C M \&$ IEEE International conference on Embedded software, pp. 2-11.

Noura, Hassan, Didier Theilliol, Jean-Christophe Ponsart, and Abbas Chamseddine (2009). Fault-tolerant control systems: Design and practical applications. Springer Science \& Business Media.

Okhravi, Hamed and Fredrick T Sheldon (2010). "Data diodes in support of trustworthy cyber infrastructure". In: Proceedings of the Sixth Annual Workshop on Cyber Security and Information Intelligence Research, pp. 1-4.

Oman, Paul, Edmund Schweitzer, and Deborah Frincke (2000). "Concerns about intrusions into remotely accessible substation controllers and SCADA systems". In: Proceedings of the TwentySeventh Annual Western Protective Relay Conference. Vol. 160. Citeseer.

Pajic, Miroslav, Insup Lee, and George J Pappas (2016). "Attackresilient state estimation for noisy dynamical systems". In: IEEE Transactions on Control of Network Systems 4.1, pp. 82-92.

Pajic, Miroslav, James Weimer, Nicola Bezzo, Paulo Tabuada, Oleg Sokolsky, Insup Lee, and George J Pappas (2014). "Robustness of attack-resilient state estimators". In: ACM/IEEE 5th International Conference on Cyber-Physical Systems (ICCPS). IEEE Computer Society, pp. 163-174. 
Palencia Gutierrez, J.C., J.J. Gutierrez Garcia, and M. Gonzalez Harbour (1998). "Best-case analysis for improving the worst-case schedulability test for distributed hard real-time systems". In: Proceedings of the $10^{\text {th }}$ Euromicro Workshop on Real-Time Systems, pp. 35-44.

Palopoli, Luigi, Claudio Pinello, Antonio Bicchi, and Alberto Sangiovanni-Vincentelli (2005). "Maximizing the stability radius of a set of systems under real-time scheduling constraints". In: Automatic Control, IEEE Transactions on 50.11, pp. 1790-1795.

Papcun, Peter, Erik Kajáti, Chao Liu, and Ray Y Zhong (2018). "Cloud-based control of industrial cyber-physical systems". In: The 48 th International Conference on Computers and Industrial Engineering (CIE).

Parno, Bryan, Jon Howell, Craig Gentry, and Mariana Raykova (2013). "Pinocchio: Nearly practical verifiable computation". In: IEEE Symposium on Security and Privacy. IEEE, pp. 238-252.

Pasqualetti, Fabio, Florian Dorfler, and Francesco Bullo (2015). "Control-theoretic methods for cyberphysical security: Geometric principles for optimal cross-layer resilient control systems". In: IEEE Control Systems Magazine 35.1, pp. 110-127.

Pasqualetti, Fabio, Florian Dörfler, and Francesco Bullo (2013). "Attack detection and identification in cyber-physical systems". In: IEEE transactions on automatic control 58.11, pp. 2715-2729.

Pei Breivold, Hongyu (2020). "Towards factories of the future: migration of industrial legacy automation systems in the cloud computing and Internet-of-things context". In: Enterprise Information Systems 14.4, pp. 542-562.

Pelle, István, János Czentye, János Dóka, and Balázs Sonkoly (2019). "Towards latency sensitive cloud native applications: A performance study on aws". In: IEEE 12th International Conference on Cloud Computing (CLOUD). IEEE, pp. 272-280. 
Pop, Paul, Petru Eles, and Zebo Peng (2003). "Schedulability analysis and optimization for the synthesis of multi-cluster distributed embedded systems". In: Design, Automation and Test in Europe Conference and Exhibition. IEEE, pp. 184-189.

- (2013). Analysis and synthesis of distributed real-time embedded systems. Springer Science \& Business Media.

Pop, Paul, Michael Lander Raagaard, Silviu S. Craciunas, and Wilfried Steiner (2016). "Design optimisation of cyber-physical distributed systems using IEEE time-sensitive networks". In: IET Cyber-Physical Systems: Theory ES Applications 1.1, pp. 86-94.

Quaglia, Davide, Riccardo Muradore, Roberto Bragantini, and Paolo Fiorini (2012). "A SystemC/Matlab co-simulation tool for networked control systems". In: Simulation Modelling Practice and Theory 23, pp. 71-86.

Quanser Inc. (2019a). 3-DOF helicopter experimental manual. https: //ww . quanser . com/products /3-dof-helicopter/. Accessed: 2021-08-31.

- (2019b). QUBE - Servo 2. https://www.quanser.com/products/ qube-servo-2/. Accessed: 2021-09-14.

Racu, Razvan and Rolf Ernst (2006). "Scheduling anomaly detection and optimization for distributed systems with preemptive tasksets". In: 12th IEEE Real-Time and Embedded Technology and Applications Symposium (RTAS). IEEE, pp. 325-334.

Rahimi, Kaveh, Abhineet Parchure, Virgilio Centeno, and Robert Broadwater (2015). "Effect of communication time-delay attacks on the performance of automatic generation control". In: North American Power Symposium (NAPS). IEEE, pp. 1-6.

Ravi, Srivaths, Anand Raghunathan, Paul Kocher, and Sunil Hattangady (2004). "Security in embedded systems: Design challenges". In: ACM Transactions on Embedded Computing Systems (TECS) 3.3, pp. 461-491. 
Redell, Ola and Martin Sanfridson (2002). "Exact Best-Case Response Time Analysis of Fixed Priority Scheduled Tasks". In: Proceedings of the $14^{\text {th }}$ Euromicro Conference on Real-Time Systems, pp. 165172.

Rehbinder, Henrik and Martin Sanfridson (2000). "Integration of offline scheduling and optimal control". In: Proceedings of the $12^{\text {th }}$ Euromicro Conference on Real-Time Systems. IEEE, pp. 137-143.

Ren, Xiaoqiang, Yilin Mo, Jie Chen, and Karl Henrik Johansson (2020). "Secure state estimation with byzantine sensors: A probabilistic approach". In: IEEE Transactions on Automatic Control 65.9 , pp. 3742-3757.

Ribeiro, Maria Isabel (2004). "Gaussian probability density functions: Properties and error characterization". In: Institute for Systems and Robotics, Lisboa, Portugal.

Roman, Rodrigo, Cristina Alcaraz, Javier Lopez, and Nicolas Sklavos (2011). "Key management systems for sensor networks in the context of the Internet of Things". In: Computers 83 Electrical Engineering 37.2, pp. 147-159.

Sagstetter, Florian, Martin Lukasiewycz, Sebastian Steinhorst, Marko Wolf, Alexandre Bouard, William R Harris, Somesh Jha, Thomas Peyrin, Axel Poschmann, and Samarjit Chakraborty (2013). "Security challenges in automotive hardware/software architecture design". In: Design, Automation 83 Test in Europe Conference 83 Exhibition (DATE). IEEE, pp. 458-463.

Sajjad, Imran, Daniel D Dunn, Rajnikant Sharma, and Ryan Gerdes (2015). "Attack mitigation in adversarial platooning using detection-based sliding mode control". In: Proceedings of the First ACM Workshop on Cyber-Physical Systems-Security and/or PrivaCy, pp. 43-53.

Samii, Soheil, Unmesh D Bordoloi, Petru Eles, and Zebo Peng (2011). "Control-quality optimization of distributed embedded control sys- 
tems with adaptive fault tolerance". In: 3rd Workshop on Adaptive and Reconfigurable Embedded Systems (APRES).

Samii, Soheil, Anton Cervin, Petru Eles, and Zebo Peng (2009). "Integrated scheduling and synthesis of control applications on distributed embedded systems". In: Design, Automation \& Test in Europe Conference $\mathcal{E}$ Exhibition. IEEE, pp. 57-62.

Samii, Soheil and Helge Zinner (2018). "Level 5 by layer 2: Timesensitive networking for autonomous vehicles". In: IEEE Communications Standards Magazine 2.2, pp. 62-68.

Sandberg, Henrik, André Teixeira, and Karl H Johansson (2010). "On security indices for state estimators in power networks". In: First Workshop on Secure Control Systems (SCS).

Sebastiani, Roberto and Patrick Trentin (2015). "OptiMathSAT: a tool for optimization modulo theories". In: International conference on computer aided verification. Springer, pp. 447-454.

Selvaraj, Jayaprakash, Gökçen Yılmaz Dayanıklı, Neelam Prabhu Gaunkar, David Ware, Ryan M Gerdes, and Mani Mina (2018). "Electromagnetic induction attacks against embedded systems". In: Proceedings of the Asia Conference on Computer and Communications Security, pp. 499-510.

Seto, Danbing, John P Lehoczky, Lui Sha, and Kang G Shin (1996). "On task schedulability in real-time control systems". In: Proceedings of the $17^{\text {th }}$ IEEE Real-Time Systems Symposium. IEEE, pp. $13-21$.

Shafique, Muhammad, Faiq Khalid, and Semeen Rehman (2018). "Intelligent security measures for smart cyber physical systems". In: 2018 21st Euromicro Conference on Digital System Design (DSD). IEEE, pp. 280-287.

Shahraeini, Mohammad, Mohammad Hossein Javidi, and Mohammad Sadegh Ghazizadeh (2010). "Comparison between communication infrastructures of centralized and decentralized wide area measure- 
ment systems". In: IEEE Transactions on Smart Grid 2.1, pp. 206211.

Shepard, Daniel P, Jahshan A Bhatti, and Todd E Humphreys (2012). "Drone hack: Spoofing attack demonstration on a civilian unmanned aerial vehicle". In: Google Scholar.

Shoukry, Yasser, Jose Araujo, Paulo Tabuada, Mani Srivastava, and Karl H Johansson (2013a). "Minimax control for cyber-physical systems under network packet scheduling attacks". In: Proceedings of the 2nd ACM international conference on High confidence networked systems. ACM, pp. 93-100.

Shoukry, Yasser, Paul Martin, Paulo Tabuada, and Mani Srivastava (2013b). "Non-invasive spoofing attacks for anti-lock braking systems". In: International Conference on Cryptographic Hardware and Embedded Systems. Springer, pp. 55-72.

Shoukry, Yasser, Alberto Puggelli, Pierluigi Nuzzo, Alberto L Sangiovanni-Vincentelli, Sanjit A Seshia, and Paulo Tabuada (2015). "Sound and complete state estimation for linear dynamical systems under sensor attacks using satisfiability modulo theory solving". In: American Control Conference (ACC). IEEE, pp. 38183823.

Skarin, Per, Johan Eker, and Karl-Erik Årzén (2020). "A cloud-enabled rate-switching MPC architecture". In: 59th IEEE Conference on Decision and Control (CDC). IEEE, pp. 3151-3158.

Slay, Jill and Michael Miller (2007). "Lessons learned from the maroochy water breach". In: International Conference on Critical Infrastructure Protection. Springer, pp. 73-82.

Smirnov, Fedor, Michael Glaß, Felix Reimann, and Jürgen Teich (2017). "Optimizing message routing and scheduling in automotive mixedcriticality time-triggered networks". In: 54th ACM/EDAC/IEEE Design Automation Conference (DAC). IEEE, pp. 1-6.

Son, Yunmok, Hocheol Shin, Dongkwan Kim, Youngseok Park, Juhwan Noh, Kibum Choi, Jungwoo Choi, and Yongdae Kim (2015). "Rock- 
ing drones with intentional sound noise on gyroscopic sensors". In: 24th USENIX Security Symposium, pp. 881-896.

Sridhar, Siddharth and Manimaran Govindarasu (2014). "Model-based attack detection and mitigation for automatic generation control". In: IEEE Transactions on Smart Grid 5.2, pp. 580-591.

Stabili, Dario, Mirco Marchetti, and Michele Colajanni (2017). "Detecting attacks to internal vehicle networks through Hamming distance". In: AEIT International Annual Conference. IEEE, pp. 16.

Stallings, William (2013). Cryptography and Network Security: Principles and Practice. 6th. Upper Saddle River, NJ, USA: Prentice Hall Press. ISBN: 0133354695, 9780133354690.

Steiner, Wilfried (2010). "An evaluation of SMT-based schedule synthesis for time-triggered multi-hop networks". In: 31st IEEE Real-Time Systems Symposium. IEEE, pp. 375-384.

- (Mar. 2011). "Synthesis of Static Communication Schedules for Mixed-Criticality Systems". In: IEEE 15th International Symposium on Object/Component/Service-Oriented Real-Time Distributed Computing Workshops 0, pp. 11-18.

Steiner, Wilfried, Günther Bauer, Brendan Hall, and Michael Paulitsch (2018). "Time-triggered Ethernet". In: Time-Triggered Communication. CRC Press, pp. 209-248.

Subashini, Subashini and Veeraruna Kavitha (2011). "A survey on security issues in service delivery models of cloud computing". In: Journal of network and computer applications 34.1, pp. 1-11.

Sun, Mu, Sibin Mohan, Lui Sha, and Carl Gunter (2009). "Addressing safety and security contradictions in cyber-physical systems". In: Proceedings of the 1st Workshop on Future Directions in CyberPhysical Systems Security (CPSSW). Citeseer.

Tămaş-Selicean, Domiţian, Paul Pop, and Wilfried Steiner (2015). "Design optimization of TTEthernet-based distributed real-time systems". In: Real-Time Systems 51.1, pp. 1-35. 
Tao, Biaoshuai and Hongjun $\mathrm{Wu}$ (2015). "Improving the biclique cryptanalysis of AES". In: Australasian Conference on Information Security and Privacy. Springer, pp. 39-56.

Taylor, Adrian, Nathalie Japkowicz, and Sylvain Leblanc (2015). "Frequency-based anomaly detection for the automotive CAN bus". In: World Congress on Industrial Control Systems Security (WCICSS). IEEE, pp. 45-49.

Teixeira, Andre, Kin Cheong Sou, Henrik Sandberg, and Karl Henrik Johansson (2015). "Secure control systems: A quantitative risk management approach". In: IEEE Control Systems Magazine 35.1, pp. 24-45.

Thiele, Lothar (2017). "Internet of Things - The Quest for Trust". In: IEEE Design \& Test 34.6, pp. 102-108.

Tian, Hui, Yuxiang Chen, Chin-Chen Chang, Hong Jiang, Yongfeng Huang, Yonghong Chen, and Jin Liu (2015). "Dynamic-hash-table based public auditing for secure cloud storage". In: IEEE Transactions on Services Computing 10.5, pp. 701-714.

Törngren, Martin, Fredrik Asplund, Saddek Bensalem, John McDermid, Roberto Passerone, Holger Pfeifer, Alberto SangiovanniVincentelli, and Bernhard Schätz (2017). "Characterization, analysis, and recommendations for exploiting the opportunities of cyberphysical systems". In: Cyber-physical systems. Elsevier, pp. 3-14.

Vestal, Steve (2007). "Preemptive scheduling of multi-criticality systems with varying degrees of execution time assurance". In: 28th IEEE International Real-Time Systems Symposium (RTSS). IEEE, pp. 239-243.

Walfish, Michael and Andrew J Blumberg (2015). "Verifying computations without reexecuting them". In: Communications of the ACM 58.2 , pp. $74-84$.

Wang, Lihui, Martin Törngren, and Mauro Onori (2015). "Current status and advancement of cyber-physical systems in manufacturing". In: Journal of Manufacturing Systems 37, pp. 517-527. 
Weber, Rolf H. (2010). "Internet of Things - New security and privacy challenges". In: Computer Law \& Security Review 26.1, pp. 23-30. ISSN: 0267-3649.

Wei, Jin and Deepa Kundur (2015). "Biologically inspired hierarchical cyber-physical multi-agent distributed control framework for sustainable smart grids". In: Cyber Physical Systems Approach to Smart Electric Power Grid. Springer, pp. 219-259.

Weiss, Gereon, Philipp Schleiss, and Christian Drabek (2016). "Towards flexible and dependable E/E-architectures for future vehicles". In: 4th International Workshop on Critical Automotive Applications: Robustness 8 Safety (CARS).

Weisstein, Eric W. (2016). Normal Difference Distribution. http : / / mathworld . wolfram . com / NormalDifferenceDistribution . html. Accessed: 2021-10-07.

Wittenmark, Björn, Johan Nilsson, and Martin Törngren (1995). "Timing Problems in Real-Time Control Systems". In: Proceedings of the American Control Conference, pp. 2000-2004.

Xie, Tao and Xiao Qin (2007). "Improving security for periodic tasks in embedded systems through scheduling". In: ACM Transactions on Embedded Computing Systems (TECS) 6.3, 20-es.

Xu, Yang, Karl Erik Årzén, Anton Cervin, Enrico Bini, and Bogdan Tanasa (2015). "Exploiting Job Response-Time Information in the Co-Design of Real-Time Control Systems". In: IEEE $21^{\text {st }}$ International Conference on Embedded and Real-Time Computing Systems and Applications (RTCSA), pp. 247-256.

Xu, Yang, Karl-Erik Årzén, Enrico Bini, and Anton Cervin (2014). "Response time driven design of control systems". In: IFAC Proceedings Volumes 47.3, pp. 6098-6104.

Yin, Shen, Hao Luo, and Steven X Ding (2013). "Real-time implementation of fault-tolerant control systems with performance optimization". In: IEEE Transactions on Industrial Electronics 61.5, pp. 2402-2411. 
Yoon, Man-Ki, Sibin Mohan, Jaesik Choi, Jung-Eun Kim, and Lui Sha (2013). "SecureCore: A multicore-based intrusion detection architecture for real-time embedded systems". In: IEEE 19th Real-Time and Embedded Technology and Applications Symposium (RTAS). IEEE, pp. 21-32.

Zhang, Fumin, Klementyna Szwaykowska, Wayne Wolf, and Vincent Mooney (2008). "Task Scheduling for Control Oriented Requirements for Cyber-Physical Systems". In: Proceedings of the $29^{\text {th }}$ IEEE Real-Time Systems Symposium, pp. 47-56.

Zhang, Licong, Dip Goswami, Reinhard Schneider, and Samarjit Chakraborty (2014). "Task- and network-level schedule co-synthesis of Ethernet-based time-triggered systems". In: 19th Asia and South Pacific Design Automation Conference (ASP-DAC). IEEE, pp. 119-124.

Zhou, Yuanbin, Soheil Samii, Petru Eles, and Zebo Peng (2021a). "Reliability-aware Scheduling and Routing for Messages in Timesensitive Networking". In: ACM Transactions on Embedded Computing Systems (TECS) 20.5, pp. 1-24.

- (2021b). "Time-Triggered Scheduling for Time-Sensitive Networking with Preemption". In: 27th Asia and South Pacific Design Automation Conference (ASP-DAC). IEEE.

Ziegenbein, Dirk and Arne Hamann (2015). "Timing-aware control software design for automotive systems". In: Proceedings of the 52nd Annual Design Automation Conference. ACM, p. 56.

Zimmer, Christopher, Balasubramanya Bhat, Frank Mueller, and Sibin Mohan (2010). "Time-based Intrusion Detection in Cyber-physical Systems". In: Proceedings of the 1st ACM/IEEE International Conference on Cyber-Physical Systems. ICCPS. ACM, pp. 109-118. 
Department of Computer and Information Science

Linköpings universitet

\section{Dissertations}

\section{Linköping Studies in Science and Technology \\ Linköping Studies in Arts and Sciences \\ Linköping Studies in Statistics \\ Linköping Studies in Information Science}

Linköping Studies in Science and Technology

No 14 Anders Haraldsson: A Program Manipulation System Based on Partial Evaluation, 1977, ISBN $91-$ 7372-144-1.

No 17 Bengt Magnhagen: Probability Based Verification of Time Margins in Digital Designs, 1977, ISBN 91-7372157-3.

No 18 Mats Cedwall: Semantisk analys av processbeskrivningar i naturligt språk, 1977, ISBN 91- 7372168-9.

No 22 Jaak Urmi: A Machine Independent LISP Compiler and its Implications for Ideal Hardware, 1978, ISBN 91-7372-188-3.

No 33 Tore Risch: Compilation of Multiple File Queries in a Meta-Database System, 1978, ISBN 91- 7372-232-4.

No 51 Erland Jungert: Synthesizing Database Structures from a User Oriented Data Model, 1980, ISBN 917372-387-8.

No 54 Sture Hägglund: Contributions to the Development of Methods and Tools for Interactive Design of Applications Software, 1980, ISBN 91-7372-404-1.

No 55 Pär Emanuelson: Performance Enhancement in a Well-Structured Pattern Matcher through Partial Evaluation, 1980, ISBN 91-7372-403-3.

No 58 Bengt Johnsson, Bertil Andersson: The HumanComputer Interface in Commercial Systems, 1981, ISBN 91-7372-414-9.

No 69 H. Jan Komorowski: A Specification of an Abstract Prolog Machine and its Application to Partial Evaluation, 1981, ISBN 91-7372-479-3.

No 71 René Reboh: Knowledge Engineering Techniques and Tools for Expert Systems, 1981, ISBN 91-7372489-0.

No 77 Östen Oskarsson: Mechanisms of Modifiability in large Software Systems, 1982, ISBN 91- 7372-527-7.

No 94 Hans Lunell: Code Generator Writing Systems, 1983, ISBN 91-7372-652-4.

No 97 Andrzej Lingas: Advances in Minimum Weight Triangulation, 1983, ISBN 91-7372-660-5.

No 109 Peter Fritzson: Towards a Distributed Programming Environment based on Incremental Compilation, 1984, ISBN 91-7372-801-2.

No 111 Erik Tengvald: The Design of Expert Planning Systems. An Experimental Operations Planning System for Turning, 1984, ISBN 91-7372- 805-5.

No 155 Christos Levcopoulos: Heuristics for Minimum Decompositions of Polygons, 1987, ISBN 91-7870133-3.

No 165 James W. Goodwin: A Theory and System for NonMonotonic Reasoning, 1987, ISBN 91-7870-183-X.

No 170 Zebo Peng: A Formal Methodology for Automated Synthesis of VLSI Systems, 1987, ISBN 91-7870-225-9.

No 174 Johan Fagerström: A Paradigm and System for Design of Distributed Systems, 1988, ISBN 91-7870301-8.
No 192 Dimiter Driankov: Towards a Many Valued Logic of Quantified Belief, 1988, ISBN 91-7870-374-3.

No 213 Lin Padgham: Non-Monotonic Inheritance for an Object Oriented Knowledge Base, 1989, ISBN 917870-485-5.

No 214 Tony Larsson: A Formal Hardware Description and Verification Method, 1989, ISBN 91-7870-517-7.

No 221 Michael Reinfrank: Fundamentals and Logical Foundations of Truth Maintenance, 1989, ISBN 917870-546-0.

No 239 Jonas Löwgren: Knowledge-Based Design Support and Discourse Management in User Interface Management Systems, 1991, ISBN 91-7870-720-X.

No 244 Henrik Eriksson: Meta-Tool Support for Knowledge Acquisition, 1991, ISBN 91-7870-746-3.

No 252 Peter Eklund: An Epistemic Approach to Interactive Design in Multiple Inheritance Hierarchies, 1991, ISBN 91-7870-784-6.

No 258 Patrick Doherty: NML3 - A Non-Monotonic Formalism with Explicit Defaults, 1991, ISBN 917870-816-8.

No 260 Nahid Shahmehri: Generalized Algorithmic Debugging, 1991, ISBN 91-7870-828-1.

No 264 Nils Dahlbäck: Representation of DiscourseCognitive and Computational Aspects, 1992, ISBN 91-7870-850-8.

No 265 Ulf Nilsson: Abstract Interpretations and Abstract Machines: Contributions to a Methodology for the Implementation of Logic Programs, 1992, ISBN 917870-858-3.

No 270 Ralph Rönnquist: Theory and Practice of Tensebound Object References, 1992, ISBN 91-7870-873-7.

No 273 Björn Fjellborg: Pipeline Extraction for VLSI Data Path Synthesis, 1992, ISBN 91-7870-880-X.

No 276 Staffan Bonnier: A Formal Basis for Horn Clause Logic with External Polymorphic Functions, 1992, ISBN 91-7870-896-6.

No 277 Kristian Sandahl: Developing Knowledge Management Systems with an Active Expert Methodology, 1992, ISBN 91-7870-897-4.

No 281 Christer Bäckström: Computational Complexity of Reasoning about Plans, 1992, ISBN 91-7870-979-2.

No 292 Mats Wirén: Studies in Incremental Natural Language Analysis, 1992, ISBN 91-7871-027-8.

No 297 Mariam Kamkar: Interprocedural Dynamic Slicing with Applications to Debugging and Testing, 1993, ISBN 91-7871-065-0.

No 302 Tingting Zhang: A Study in Diagnosis Using Classification and Defaults, 1993, ISBN 91-7871-0782.

No 312 Arne Jönsson: Dialogue Management for Natural Language Interfaces - An Empirical Approach, 1993, ISBN 91-7871-110-X.

No 338 Simin Nadjm-Tehrani: Reactive Systems in Physical Environments: Compositional Modelling and Framework for Verification, 1994, ISBN 91-7871-237-8. 
No 371 Bengt Savén: Business Models for Decision Support and Learning. A Study of Discrete-Event Manufacturing Simulation at Asea/ABB 1968-1993, 1995, ISBN 91-7871-494-X.

No 375 Ulf Söderman: Conceptual Modelling of Mode Switching Physical Systems, 1995, ISBN 91-7871-5164.

No 383 Andreas Kågedal: Exploiting Groundness in Logic Programs, 1995, ISBN 91-7871-538-5.

No 396 George Fodor: Ontological Control, Description, Identification and Recovery from Problematic Control Situations, 1995, ISBN 91-7871-603-9.

No 413 Mikael Pettersson: Compiling Natural Semantics, 1995, ISBN 91-7871-641-1.

No 414 Xinli Gu: RT Level Testability Improvement by Testability Analysis and Transformations, 1996, ISBN 91-7871-654-3.

No 416 Hua Shu: Distributed Default Reasoning, 1996, ISBN 91-7871-665-9.

No 429 Jaime Villegas: Simulation Supported Industrial Training from an Organisational Learning Perspective - Development and Evaluation of the SSIT Method, 1996, ISBN 91-7871-700-0.

No 431 Peter Jonsson: Studies in Action Planning: Algorithms and Complexity, 1996, ISBN 91-7871-7043.

No 437 Johan Boye: Directional Types in Logic Programming, 1996, ISBN 91-7871-725-6.

No 439 Cecilia Sjöberg: Activities, Voices and Arenas: Participatory Design in Practice, 1996, ISBN 91-7871728-0.

No 448 Patrick Lambrix: Part-Whole Reasoning in Description Logics, 1996, ISBN 91-7871-820-1.

No 452 Kjell Orsborn: On Extensible and Object-Relational Database Technology for Finite Element Analysis Applications, 1996, ISBN 91-7871-827-9.

No 459 Olof Johansson: Development Environments for Complex Product Models, 1996, ISBN 91-7871-855-4.

No 461 Lena Strömbäck: User-Defined Constructions in Unification-Based Formalisms, 1997, ISBN 91-7871857-0.

No 462 Lars Degerstedt: Tabulation-based Logic Programming: A Multi-Level View of Query Answering, 1996, ISBN 91-7871-858-9.

No 475 Fredrik Nilsson: Strategi och ekonomisk styrning En studie av hur ekonomiska styrsystem utformas och används efter företagsförvärv, 1997, ISBN 917871-914-3.

No 480 Mikael Lindvall: An Empirical Study of Requirements-Driven Impact Analysis in Object-Oriented Software Evolution, 1997, ISBN 91-7871-927-5.

No 485 Göran Forslund: Opinion-Based Systems: The Cooperative Perspective on Knowledge-Based Decision Support, 1997, ISBN 91-7871-938-0.

No 494 Martin Sköld: Active Database Management Systems for Monitoring and Control, 1997, ISBN 917219-002-7.

No 495 Hans Olsén: Automatic Verification of Petri Nets in a CLP framework, 1997, ISBN 91-7219-011-6.

No 498 Thomas Drakengren: Algorithms and Complexity for Temporal and Spatial Formalisms, 1997, ISBN 917219-019-1.

No 502 Jakob Axelsson: Analysis and Synthesis of Heterogeneous Real-Time Systems, 1997, ISBN 91-7219-035-3.
No 503 Johan Ringström: Compiler Generation for DataParallel Programming Languages from Two-Level Semantics Specifications, 1997, ISBN 91-7219-045-0.

No 512 Anna Moberg: Närhet och distans - Studier av kommunikationsmönster i satellitkontor och flexibla kontor, 1997, ISBN 91-7219-119-8.

No 520 Mikael Ronström: Design and Modelling of a Parallel Data Server for Telecom Applications, 1998, ISBN 91-7219-169-4.

No 522 Niclas Ohlsson: Towards Effective Fault Prevention - An Empirical Study in Software Engineering, 1998, ISBN 91-7219-176-7.

No 526 Joachim Karlsson: A Systematic Approach for Prioritizing Software Requirements, 1998, ISBN 917219-184-8.

No 530 Henrik Nilsson: Declarative Debugging for Lazy Functional Languages, 1998, ISBN 91-7219-197-X.

No 555 Jonas Hallberg: Timing Issues in High-Level Synthesis, 1998, ISBN 91-7219-369-7.

No 561 Ling Lin: Management of 1-D Sequence Data - From Discrete to Continuous, 1999, ISBN 91-7219-402-2.

No 563 Eva L Ragnemalm: Student Modelling based on Collaborative Dialogue with a Learning Companion, 1999, ISBN 91-7219-412-X.

No 567 Jörgen Lindström: Does Distance matter? On geographical dispersion in organisations, 1999, ISBN 917219-439-1.

No 582 Vanja Josifovski: Design, Implementation and Evaluation of a Distributed Mediator System for Data Integration, 1999, ISBN 91-7219-482-0.

No 589 Rita Kovordányi: Modeling and Simulating Inhibitory Mechanisms in Mental Image Reinterpretation - Towards Cooperative HumanComputer Creativity, 1999, ISBN 91-7219-506-1.

No 592 Mikael Ericsson: Supporting the Use of Design Knowledge - An Assessment of Commenting Agents, 1999, ISBN 91-7219-532-0.

No 593 Lars Karlsson: Actions, Interactions and Narratives, 1999, ISBN 91-7219-534-7.

No 594 C. G. Mikael Johansson: Social and Organizational Aspects of Requirements Engineering Methods - A practice-oriented approach, 1999, ISBN 91-7219-541$\mathrm{X}$.

No 595 Jörgen Hansson: Value-Driven Multi-Class Overload Management in Real-Time Database Systems, 1999, ISBN 91-7219-542-8.

No 596 Niklas Hallberg: Incorporating User Values in the Design of Information Systems and Services in the Public Sector: A Methods Approach, 1999, ISBN 917219-543-6.

No 597 Vivian Vimarlund: An Economic Perspective on the Analysis of Impacts of Information Technology: From Case Studies in Health-Care towards General Models and Theories, 1999, ISBN 91-7219-544-4.

No 598 Johan Jenvald: Methods and Tools in ComputerSupported Taskforce Training, 1999, ISBN 91-7219547-9.

No 607 Magnus Merkel: Understanding and enhancing translation by parallel text processing, 1999, ISBN 917219-614-9.

No 611 Silvia Coradeschi: Anchoring symbols to sensory data, 1999, ISBN 91-7219-623-8.

No 613 Man Lin: Analysis and Synthesis of Reactive Systems: A Generic Layered Architecture Perspective, 1999, ISBN 91-7219-630-0. 
No 618 Jimmy Tjäder: Systemimplementering i praktiken En studie av logiker i fyra projekt, 1999, ISBN 917219-657-2.

No 627 Vadim Engelson: Tools for Design, Interactive Simulation, and Visualization of Object-Oriented Models in Scientific Computing, 2000, ISBN 91-7219709-9.

No 637 Esa Falkenroth: Database Technology for Control and Simulation, 2000, ISBN 91-7219-766-8.

No 639 Per-Arne Persson: Bringing Power and Knowledge Together: Information Systems Design for Autonomy and Control in Command Work, 2000, ISBN 91-7219796-X.

No 660 Erik Larsson: An Integrated System-Level Design for Testability Methodology, 2000, ISBN 91-7219-890-7.

No 688 Marcus Bjäreland: Model-based Execution Monitoring, 2001, ISBN 91-7373-016-5.

No 689 Joakim Gustafsson: Extending Temporal Action Logic, 2001, ISBN 91-7373-017-3.

No 720 Carl-Johan Petri: Organizational Information Provision - Managing Mandatory and Discretionary Use of Information Technology, 2001, ISBN 91-7373-1269.

No 724 Paul Scerri: Designing Agents for Systems with Adjustable Autonomy, 2001, ISBN 91-7373-207-9.

No 725 Tim Heyer: Semantic Inspection of Software Artifacts: From Theory to Practice, 2001, ISBN 917373-208-7.

No 726 Pär Carlshamre: A Usability Perspective on Requirements Engineering - From Methodology to Product Development, 2001, ISBN 91-7373-212-5.

No 732 Juha Takkinen: From Information Management to Task Management in Electronic Mail, 2002, ISBN 917373-258-3.

No 745 Johan Åberg: Live Help Systems: An Approach to Intelligent Help for Web Information Systems, 2002, ISBN 91-7373-311-3.

No 746 Rego Granlund: Monitoring Distributed Teamwork Training, 2002, ISBN 91-7373-312-1.

No 757 Henrik André-Jönsson: Indexing Strategies for Time Series Data, 2002, ISBN 917373-346-6.

No 747 Anneli Hagdahl: Development of IT-supported Interorganisational Collaboration - A Case Study in the Swedish Public Sector, 2002, ISBN 91-7373-314-8.

No 749 Sofie Pilemalm: Information Technology for NonProfit Organisations - Extended Participatory Design of an Information System for Trade Union Shop Stewards, 2002, ISBN 91-7373-318-0.

No 765 Stefan Holmlid: Adapting users: Towards a theory of use quality, 2002, ISBN 91-7373-397-0.

No 771 Magnus Morin: Multimedia Representations of Distributed Tactical Operations, 2002, ISBN 91-7373-4217.

No 772 Pawel Pietrzak: A Type-Based Framework for Locating Errors in Constraint Logic Programs, 2002, ISBN 91-7373-422-5.

No 758 Erik Berglund: Library Communication Among Programmers Worldwide, 2002, ISBN 91-7373-349-0.

No 774 Choong-ho Yi: Modelling Object-Oriented Dynamic Systems Using a Logic-Based Framework, 2002, ISBN 91-7373-424-1.

No 779 Mathias Broxvall: A Study in the Computational Complexity of Temporal Reasoning, 2002, ISBN 917373-440-3.
No 793 Asmus Pandikow: A Generic Principle for Enabling Interoperability of Structured and Object-Oriented Analysis and Design Tools, 2002, ISBN 91-7373-479-9.

No 785 Lars Hult: Publika Informationstjänster. En studie av den Internetbaserade encyklopedins bruksegenskaper, 2003, ISBN 91-7373-461-6.

No 800 Lars Taxén: A Framework for the Coordination of Complex Systems' Development, 2003, ISBN 917373-604-X.

No 808 Klas Gäre: Tre perspektiv på förväntningar och förändringar $i$ samband med införande av informationssystem, 2003, ISBN 91-7373-618-X.

No 821 Mikael Kindborg: Concurrent Comics programming of social agents by children, 2003, ISBN 91-7373-651-1.

No 823 Christina Ölvingson: On Development of Information Systems with GIS Functionality in Public Health Informatics: A Requirements Engineering Approach, 2003, ISBN 91-7373-656-2.

No 828 Tobias Ritzau: Memory Efficient Hard Real-Time Garbage Collection, 2003, ISBN 91-7373-666-X.

No 833 Paul Pop: Analysis and Synthesis of Communication-Intensive Heterogeneous Real-Time Systems, 2003, ISBN 91-7373-683-X.

No 852 Johan Moe: Observing the Dynamic Behaviour of Large Distributed Systems to Improve Development and Testing - An Empirical Study in Software Engineering, 2003, ISBN 91-7373-779-8.

No 867 Erik Herzog: An Approach to Systems Engineering Tool Data Representation and Exchange, 2004, ISBN 91-7373-929-4.

No 872 Aseel Berglund: Augmenting the Remote Control: Studies in Complex Information Navigation for Digital TV, 2004, ISBN 91-7373-940-5.

No 869 Jo Skåmedal: Telecommuting's Implications on Travel and Travel Patterns, 2004, ISBN 91-7373-935-9.

No 870 Linda Askenäs: The Roles of IT - Studies of Organising when Implementing and Using Enterprise Systems, 2004, ISBN 91-7373-936-7.

No 874 Annika Flycht-Eriksson: Design and Use of Ontologies in Information-Providing Dialogue Systems, 2004, ISBN 91-7373-947-2.

No 873 Peter Bunus: Debugging Techniques for EquationBased Languages, 2004, ISBN 91-7373-941-3.

No 876 Jonas Mellin: Resource-Predictable and Efficient Monitoring of Events, 2004, ISBN 91-7373-956-1.

No 883 Magnus Bång: Computing at the Speed of Paper: Ubiquitous Computing Environments for Healthcare Professionals, 2004, ISBN 91-7373-971-5.

No 882 Robert Eklund: Disfluency in Swedish humanhuman and human-machine travel booking dialogues, 2004, ISBN 91-7373-966-9.

No 887 Anders Lindström: English and other Foreign Linguistic Elements in Spoken Swedish. Studies of Productive Processes and their Modelling using Finite-State Tools, 2004, ISBN 91-7373-981-2.

No 889 Zhiping Wang: Capacity-Constrained Production-inventory systems - Modelling and Analysis in both a traditional and an e-business context, 2004, ISBN 9185295-08-6

No 893 Pernilla Qvarfordt: Eyes on Multimodal Interaction, 2004, ISBN 91-85295-30-2.

No 910 Magnus Kald: In the Borderland between Strategy and Management Control - Theoretical Framework and Empirical Evidence, 2004, ISBN 91-85295-82-5. 
No 918 Jonas Lundberg: Shaping Electronic News: Genre Perspectives on Interaction Design, 2004, ISBN 9185297-14-3.

No 900 Mattias Arvola: Shades of use: The dynamics of interaction design for sociable use, 2004, ISBN 9185295-42-6.

No 920 Luis Alejandro Cortés: Verification and Scheduling Techniques for Real-Time Embedded Systems, 2004, ISBN 91-85297-21-6.

No 929 Diana Szentivanyi: Performance Studies of FaultTolerant Middleware, 2005, ISBN 91-85297-58-5.

No 933 Mikael Cäker: Management Accounting as Constructing and Opposing Customer Focus: Three Case Studies on Management Accounting and Customer Relations, 2005, ISBN 91-85297-64-X.

No 937 Jonas Kvarnström: TALplanner and Other Extensions to Temporal Action Logic, 2005, ISBN 9185297-75-5.

No 938 Bourhane Kadmiry: Fuzzy Gain-Scheduled Visual Servoing for Unmanned Helicopter, 2005, ISBN 9185297-76-3.

No 945 Gert Jervan: Hybrid Built-In Self-Test and Test Generation Techniques for Digital Systems, 2005, ISBN 91-85297-97-6.

No 946 Anders Arpteg: Intelligent Semi-Structured Information Extraction, 2005, ISBN 91-85297-98-4.

No 947 Ola Angelsmark: Constructing Algorithms for Constraint Satisfaction and Related Problems - Methods and Applications, 2005, ISBN 91-85297-99-2.

No 963 Calin Curescu: Utility-based Optimisation of Resource Allocation for Wireless Networks, 2005, ISBN 91-85457-07-8.

No 972 Björn Johansson: Joint Control in Dynamic Situations, 2005, ISBN 91-85457-31-0.

No 974 Dan Lawesson: An Approach to Diagnosability Analysis for Interacting Finite State Systems, 2005, ISBN 91-85457-39-6.

No 979 Claudiu Duma: Security and Trust Mechanisms for Groups in Distributed Services, 2005, ISBN 91-8545754-X.

No 983 Sorin Manolache: Analysis and Optimisation of Real-Time Systems with Stochastic Behaviour, 2005, ISBN 91-85457-60-4.

No 986 Yuxiao Zhao: Standards-Based Application Integration for Business-to-Business Communications, 2005, ISBN 91-85457-66-3.

No 1004 Patrik Haslum: Admissible Heuristics for Automated Planning, 2006, ISBN 91-85497-28-2.

No 1005 Aleksandra Tešanovic: Developing Reusable and Reconfigurable Real-Time Software using Aspects and Components, 2006, ISBN 91-85497-29-0.

No 1008 David Dinka: Role, Identity and Work: Extending the design and development agenda, 2006, ISBN 9185497-42-8.

No 1009 Iakov Nakhimovski: Contributions to the Modeling and Simulation of Mechanical Systems with Detailed Contact Analysis, 2006, ISBN 91-85497-43-X.

No 1013 Wilhelm Dahllöf: Exact Algorithms for Exact Satisfiability Problems, 2006, ISBN 91-85523-97-6.

No 1016 Levon Saldamli: PDEModelica - A High-Level Language for Modeling with Partial Differential Equations, 2006, ISBN 91-85523-84-4.

No 1017 Daniel Karlsson: Verification of Component-based Embedded System Designs, 2006, ISBN 91-85523-79-8
No 1018 Ioan Chisalita: Communication and Networking Techniques for Traffic Safety Systems, 2006, ISBN 9185523-77-1.

No 1019 Tarja Susi: The Puzzle of Social Activity - The Significance of Tools in Cognition and Cooperation, 2006, ISBN 91-85523-71-2.

No 1021 Andrzej Bednarski: Integrated Optimal Code Generation for Digital Signal Processors, 2006, ISBN 9185523-69-0.

No 1022 Peter Aronsson: Automatic Parallelization of Equation-Based Simulation Programs, 2006, ISBN 9185523-68-2.

No 1030 Robert Nilsson: A Mutation-based Framework for Automated Testing of Timeliness, 2006, ISBN 9185523-35-6.

No 1034 Jon Edvardsson: Techniques for Automatic Generation of Tests from Programs and Specifications, 2006, ISBN 91-85523-31-3.

No 1035 Vaida Jakoniene: Integration of Biological Data, 2006, ISBN 91-85523-28-3.

No 1045 Genevieve Gorrell: Generalized Hebbian Algorithms for Dimensionality Reduction in Natural Language Processing, 2006, ISBN 91-85643-88-2.

No 1051 Yu-Hsing Huang: Having a New Pair of Glasses Applying Systemic Accident Models on Road Safety, 2006, ISBN 91-85643-64-5.

No 1054 Åsa Hedenskog: Perceive those things which cannot be seen - A Cognitive Systems Engineering perspective on requirements management, 2006, ISBN 91-85643-57-2.

No 1061 Cécile Åberg: An Evaluation Platform for Semantic Web Technology, 2007, ISBN 91-85643-31-9.

No 1073 Mats Grindal: Handling Combinatorial Explosion in Software Testing, 2007, ISBN 978-91-85715-74-9.

No 1075 Almut Herzog: Usable Security Policies for Runtime Environments, 2007, ISBN 978-91-85715-65-7.

No 1079 Magnus Wahlström: Algorithms, measures, and upper bounds for Satisfiability and related problems, 2007, ISBN 978-91-85715-55-8.

No 1083 Jesper Andersson: Dynamic Software Architectures, 2007, ISBN 978-91-85715-46-6.

No 1086 Ulf Johansson: Obtaining Accurate and Comprehensible Data Mining Models - An Evolutionary Approach, 2007, ISBN 978-91-85715-34-3.

No 1089 Traian Pop: Analysis and Optimisation of Distributed Embedded Systems with Heterogeneous Scheduling Policies, 2007, ISBN 978-91-85715-27-5.

No 1091 Gustav Nordh: Complexity Dichotomies for CSPrelated Problems, 2007, ISBN 978-91-85715-20-6.

No 1106 Per Ola Kristensson: Discrete and Continuous Shape Writing for Text Entry and Control, 2007, ISBN 97891-85831-77-7.

No 1110 He Tan: Aligning Biomedical Ontologies, 2007, ISBN 978-91-85831-56-2.

No 1112 Jessica Lindblom: Minding the body - Interacting socially through embodied action, 2007, ISBN 978-9185831-48-7.

No 1113 Pontus Wärnestål: Dialogue Behavior Management in Conversational Recommender Systems, 2007, ISBN 978-91-85831-47-0.

No 1120 Thomas Gustafsson: Management of Real-Time Data Consistency and Transient Overloads in Embedded Systems, 2007, ISBN 978-91-85831-33-3. 
No 1127 Alexandru Andrei: Energy Efficient and Predictable Design of Real-time Embedded Systems, 2007, ISBN 978-91-85831-06-7.

No 1139 Per Wikberg: Eliciting Knowledge from Experts in Modeling of Complex Systems: Managing Variation and Interactions, 2007, ISBN 978-91-85895-66-3.

No 1143 Mehdi Amirijoo: QoS Control of Real-Time Data Services under Uncertain Workload, 2007, ISBN 97891-85895-49-6.

No 1150 Sanny Syberfeldt: Optimistic Replication with Forward Conflict Resolution in Distributed Real-Time Databases, 2007, ISBN 978-91-85895-27-4.

No 1155 Beatrice Alenljung: Envisioning a Future Decision Support System for Requirements Engineering - A Holistic and Human-centred Perspective, 2008, ISBN 978-91-85895-11-3.

No 1156 Artur Wilk: Types for XML with Application to Xcerpt, 2008, ISBN 978-91-85895-08-3.

No 1183 Adrian Pop: Integrated Model-Driven Development Environments for Equation-Based Object-Oriented Languages, 2008, ISBN 978-91-7393-895-2.

No 1185 Jörgen Skågeby: Gifting Technologies Ethnographic Studies of End-users and Social Media Sharing, 2008, ISBN 978-91-7393-892-1.

No 1187 Imad-Eldin Ali Abugessaisa: Analytical tools and information-sharing methods supporting road safety organizations, 2008, ISBN 978-91-7393-887-7.

No 1204 H. Joe Steinhauer: A Representation Scheme for Description and Reconstruction of Object Configurations Based on Qualitative Relations, 2008, ISBN 978-91-7393-823-5.

No 1222 Anders Larsson: Test Optimization for Core-based System-on-Chip, 2008, ISBN 978-91-7393-768-9.

No 1238 Andreas Borg: Processes and Models for Capacity Requirements in Telecommunication Systems, 2009, ISBN 978-91-7393-700-9.

No 1240 Fredrik Heintz: DyKnow: A Stream-Based Knowledge Processing Middleware Framework, 2009, ISBN 978-91-7393-696-5.

No 1241 Birgitta Lindström: Testability of Dynamic RealTime Systems, 2009, ISBN 978-91-7393-695-8.

No 1244 Eva Blomqvist: Semi-automatic Ontology Construction based on Patterns, 2009, ISBN 978-91-7393-683-5.

No 1249 Rogier Woltjer: Functional Modeling of Constraint Management in Aviation Safety and Command and Control, 2009, ISBN 978-91-7393-659-0.

No 1260 Gianpaolo Conte: Vision-Based Localization and Guidance for Unmanned Aerial Vehicles, 2009, ISBN 978-91-7393-603-3.

No 1262 AnnMarie Ericsson: Enabling Tool Support for Formal Analysis of ECA Rules, 2009, ISBN 978-91-7393598-2.

No 1266 Jiri Trnka: Exploring Tactical Command and Control: A Role-Playing Simulation Approach, 2009, ISBN 978-91-7393-571-5.

No 1268 Bahlol Rahimi: Supporting Collaborative Work through ICT - How End-users Think of and Adopt Integrated Health Information Systems, 2009, ISBN 978-91-7393-550-0.

No 1274 Fredrik Kuivinen: Algorithms and Hardness Results for Some Valued CSPs, 2009, ISBN 978-91-7393-525-8.

No 1281 Gunnar Mathiason: Virtual Full Replication for Scalable Distributed Real-Time Databases, 2009, ISBN 978-91-7393-503-6.
No 1290 Viacheslav Izosimov: Scheduling and Optimization of Fault-Tolerant Distributed Embedded Systems, 2009, ISBN 978-91-7393-482-4.

No 1294 Johan Thapper: Aspects of a Constraint Optimisation Problem, 2010, ISBN 978-91-7393-464-0.

No 1306 Susanna Nilsson: Augmentation in the Wild: User Centered Development and Evaluation of Augmented Reality Applications, 2010, ISBN 978-917393-416-9.

No 1313 Christer Thörn: On the Quality of Feature Models, 2010, ISBN 978-91-7393-394-0.

No 1321 Zhiyuan He: Temperature Aware and DefectProbability Driven Test Scheduling for System-onChip, 2010, ISBN 978-91-7393-378-0.

No 1333 David Broman: Meta-Languages and Semantics for Equation-Based Modeling and Simulation, 2010, ISBN 978-91-7393-335-3.

No 1337 Alexander Siemers: Contributions to Modelling and Visualisation of Multibody Systems Simulations with Detailed Contact Analysis, 2010, ISBN 978-91-7393317-9.

No 1354 Mikael Asplund: Disconnected Discoveries: Availability Studies in Partitioned Networks, 2010, ISBN 978-91-7393-278-3.

No 1359 Jana Rambusch: Mind Games Extended: Understanding Gameplay as Situated Activity, 2010, ISBN 978-91-7393-252-3.

No 1373 Sonia Sangari: Head Movement Correlates to Focus Assignment in Swedish, 2011, ISBN 978-91-7393-1540 .

No 1374 Jan-Erik Källhammer: Using False Alarms when Developing Automotive Active Safety Systems, 2011, ISBN 978-91-7393-153-3.

No 1375 Mattias Eriksson: Integrated Code Generation, 2011, ISBN 978-91-7393-147-2.

No 1381 Ola Leifler: Affordances and Constraints of Intelligent Decision Support for Military Command and Control - Three Case Studies of Support Systems, 2011, ISBN 978-91-7393-133-5.

No 1386 Soheil Samii: Quality-Driven Synthesis and Optimization of Embedded Control Systems, 2011, ISBN 978-91-7393-102-1.

No 1419 Erik Kuiper: Geographic Routing in Intermittentlyconnected Mobile Ad Hoc Networks: Algorithms and Performance Models, 2012, ISBN 978-91-7519981-8.

No 1451 Sara Stymne: Text Harmonization Strategies for Phrase-Based Statistical Machine Translation, 2012, ISBN 978-91-7519-887-3.

No 1455 Alberto Montebelli: Modeling the Role of Energy Management in Embodied Cognition, 2012, ISBN 978-91-7519-882-8.

No 1465 Mohammad Saifullah: Biologically-Based Interactive Neural Network Models for Visual Attention and Object Recognition, 2012, ISBN 978-91-7519-838-5.

No 1490 Tomas Bengtsson: Testing and Logic Optimization Techniques for Systems on Chip, 2012, ISBN 978-917519-742-5.

No 1481 David Byers: Improving Software Security by Preventing Known Vulnerabilities, 2012, ISBN 97891-7519-784-5.

No 1496 Tommy Färnqvist: Exploiting Structure in CSPrelated Problems, 2013, ISBN 978-91-7519-711-1. 
No 1503 John Wilander: Contributions to Specification, Implementation, and Execution of Secure Software, 2013, ISBN 978-91-7519-681-7.

No 1506 Magnus Ingmarsson: Creating and Enabling the Useful Service Discovery Experience, 2013, ISBN 97891-7519-662-6.

No 1547 Wladimir Schamai: Model-Based Verification of Dynamic System Behavior against Requirements: Method, Language, and Tool, 2013, ISBN 978-917519-505-6.

No 1551 Henrik Svensson: Simulations, 2013, ISBN 978-917519-491-2.

No 1559 Sergiu Rafiliu: Stability of Adaptive Distributed Real-Time Systems with Dynamic Resource Management, 2013, ISBN 978-91-7519-471-4.

No 1581 Usman Dastgeer: Performance-aware Component Composition for GPU-based Systems, 2014, ISBN 978-91-7519-383-0.

No 1602 Cai Li: Reinforcement Learning of Locomotion based on Central Pattern Generators, 2014, ISBN 978-917519-313-7.

No 1652 Roland Samlaus: An Integrated Development Environment with Enhanced Domain-Specific Interactive Model Validation, 2015, ISBN 978-917519-090-7.

No 1663 Hannes Uppman: On Some Combinatorial Optimization Problems: Algorithms and Complexity, 2015, ISBN 978-91-7519-072-3.

No 1664 Martin Sjölund: Tools and Methods for Analysis, Debugging, and Performance Improvement of Equation-Based Models, 2015, ISBN 978-91-7519-071-6.

No 1666 Kristian Stavåker: Contributions to Simulation of Modelica Models on Data-Parallel Multi-Core Architectures, 2015, ISBN 978-91-7519-068-6.

No 1680 Adrian Lifa: Hardware/Software Codesign of Embedded Systems with Reconfigurable and Heterogeneous Platforms, 2015, ISBN 978-91-7519-0402.

No 1685 Bogdan Tanasa: Timing Analysis of Distributed Embedded Systems with Stochastic Workload and Reliability Constraints, 2015, ISBN 978-91-7519-022-8.

No 1691 Håkan Warnquist: Troubleshooting Trucks Automated Planning and Diagnosis, 2015, ISBN 97891-7685-993-3.

No 1702 Nima Aghaee: Thermal Issues in Testing of Advanced Systems on Chip, 2015, ISBN 978-91-7685949-0.

No 1715 Maria Vasilevskaya: Security in Embedded Systems: A Model-Based Approach with Risk Metrics, 2015, ISBN 978-91-7685-917-9.

No 1729 Ke Jiang: Security-Driven Design of Real-Time Embedded System, 2016, ISBN 978-91-7685-884-4.

No 1733 Victor Lagerkvist: Strong Partial Clones and the Complexity of Constraint Satisfaction Problems: Limitations and Applications, 2016, ISBN 978-91-7685856-1.

No 1734 Chandan Roy: An Informed System Development Approach to Tropical Cyclone Track and Intensity Forecasting, 2016, ISBN 978-91-7685-854-7.

No 1746 Amir Aminifar: Analysis, Design, and Optimization of Embedded Control Systems, 2016, ISBN 978-917685-826-4.

No 1747 Ekhiotz Vergara: Energy Modelling and Fairness for Efficient Mobile Communication, 2016, ISBN 978-917685-822-6.
No 1748 Dag Sonntag: Chain Graphs - Interpretations, Expressiveness and Learning Algorithms, 2016, ISBN 978-91-7685-818-9.

No 1768 Anna Vapen: Web Authentication using ThirdParties in Untrusted Environments, 2016, ISBN 97891-7685-753-3.

No 1778 Magnus Jandinger: On a Need to Know Basis: A Conceptual and Methodological Framework for Modelling and Analysis of Information Demand in an Enterprise Context, 2016, ISBN 978-91-7685-713-7.

No 1798 Rahul Hiran: Collaborative Network Security: Targeting Wide-area Routing and Edge-network Attacks, 2016, ISBN 978-91-7685-662-8.

No 1813 Nicolas Melot: Algorithms and Framework for Energy Efficient Parallel Stream Computing on Many-Core Architectures, 2016, ISBN 978-91-7685623-9.

No 1823 Amy Rankin: Making Sense of Adaptations: Resilience in High-Risk Work, 2017, ISBN 978-917685-596-6.

No 1831 Lisa Malmberg: Building Design Capability in the Public Sector: Expanding the Horizons of Development, 2017, ISBN 978-91-7685-585-0.

No 1851 Marcus Bendtsen: Gated Bayesian Networks, 2017, ISBN 978-91-7685-525-6.

No 1852 Zlatan Dragisic: Completion of Ontologies and Ontology Networks, 2017, ISBN 978-91-7685-522-5.

No 1854 Meysam Aghighi: Computational Complexity of some Optimization Problems in Planning, 2017, ISBN 978-91-7685-519-5.

No 1863 Simon Ståhlberg: Methods for Detecting Unsolvable Planning Instances using Variable Projection, 2017, ISBN 978-91-7685-498-3.

No 1879 Karl Hammar: Content Ontology Design Patterns: Qualities, Methods, and Tools, 2017, ISBN 978-917685-454-9.

No 1887 Ivan Ukhov: System-Level Analysis and Design under Uncertainty, 2017, ISBN 978-91-7685-426-6.

No 1891 Valentina Ivanova: Fostering User Involvement in Ontology Alignment and Alignment Evaluation, 2017, ISBN 978-91-7685-403-7.

No 1902 Vengatanathan Krishnamoorthi: Efficient HTTPbased Adaptive Streaming of Linear and Interactive Videos, 2018, ISBN 978-91-7685-371-9.

No 1903 Lu Li: Programming Abstractions and Optimization Techniques for GPU-based Heterogeneous Systems, 2018, ISBN 978-91-7685-370-2.

No 1913 Jonas Rybing: Studying Simulations with Distributed Cognition, 2018, ISBN 978-91-7685-348-1.

No 1936 Leif Jonsson: Machine Learning-Based Bug Handling in Large-Scale Software Development, 2018, ISBN 978-91-7685-306-1.

No 1964 Arian Maghazeh: System-Level Design of GPUBased Embedded Systems, 2018, ISBN 978-91-7685175-3.

No 1967 Mahder Gebremedhin: Automatic and Explicit Parallelization Approaches for Equation Based Mathematical Modeling and Simulation, 2019, ISBN 978-91-7685-163-0.

No 1984 Anders Andersson: Distributed Moving Base Driving Simulators - Technology, Performance, and Requirements, 2019, ISBN 978-91-7685-090-9.

No 1993 Ulf Kargén: Scalable Dynamic Analysis of Binary Code, 2019, ISBN 978-91-7685-049-7. 
No 2001 Tim Overkamp: How Service Ideas Are Implemented: Ways of Framing and Addressing Service Transformation, 2019, ISBN 978-91-7685-025-1.

No 2006 Daniel de Leng: Robust Stream Reasoning Under Uncertainty, 2019, ISBN 978-91-7685-013-8.

No 2048 Biman Roy: Applications of Partial Polymorphisms in (Fine-Grained) Complexity of Constraint Satisfaction Problems, 2020, ISBN 978-91-7929-898-2.

No 2051 Olov Andersson: Learning to Make Safe Real-Time Decisions Under Uncertainty for Autonomous Robots, 2020, ISBN 978-91-7929-889-0.

No 2065 Vanessa Rodrigues: Designing for Resilience: Navigating Change in Service Systems, 2020, ISBN 978-91-7929-867-8.

No 2082 Robin Kurtz: Contributions to Semantic Dependency Parsing: Search, Learning, and Application, 2020, ISBN 978-91-7929-822-7.

No 2108 Shanai Ardi: Vulnerability and Risk Analysis Methods and Application in Large Scale Development of Secure Systems, 2021, ISBN 978-917929-744-2.

No 2125 Zeinab Ganjei: Parameterized Verification of Synchronized Concurrent Programs, 2021, ISBN 97891-7929-697-1.

No 2153 Robin Keskisärkkä: Complex Event Processing under Uncertainty in RDF Stream Processing, 2021, ISBN 978-91-7929-621-6.

No 2168 Rouhollah Mahfouzi: Security-Aware Design of Cyber-Physical Systems for Control Applications, 2021, ISBN 978-91-7929-021-4.

Linköping Studies in Arts and Sciences

No504 Ing-Marie Jonsson: Social and Emotional Characteristics of Speech-based In-Vehicle Information Systems: Impact on Attitude and Driving Behaviour, 2009, ISBN 978-91-7393-478-7.

No 586 Fabian Segelström: Stakeholder Engagement for Service Design: How service designers identify and communicate insights, 2013, ISBN 978-91-7519-554-4.

No 618 Johan Blomkvist: Representing Future Situations of Service: Prototyping in Service Design, 2014, ISBN 978-91-7519-343-4.

No 620 Marcus Mast: Human-Robot Interaction for SemiAutonomous Assistive Robots, 2014, ISBN 978-917519-319-9.

No677 Peter Berggren: Assessing Shared Strategic Understanding, 2016, ISBN 978-91-7685-786-1.

No 695 Mattias Forsblad: Distributed cognition in home environments: The prospective memory and cognitive practices of older adults, 2016, ISBN 97891-7685-686-4.

No 787 Sara Nygårdhs: Adaptive behaviour in traffic: An individual road user perspective, 2020, ISBN 978-917929-857-9.

No 811 Sam Thellman: Social Robots as Intentional Agents, 2021, ISBN 978-91-7929-008-5.

\section{Linköping Studies in Statistics}

No 9 Davood Shahsavani: Computer Experiments Designed to Explore and Approximate Complex Deterministic Models, 2008, ISBN 978-91-7393-976-8.

No 10 Karl Wahlin: Roadmap for Trend Detection and Assessment of Data Quality, 2008, ISBN 978-91-7393$792-4$.
No 11 Oleg Sysoev: Monotonic regression for large multivariate datasets, 2010, ISBN 978-91-7393-412-1.

No 13 Agné Burauskaite-Harju: Characterizing Temporal Change and Inter-Site Correlations in Daily and Subdaily Precipitation Extremes, 2011, ISBN 978-91-7393110-6.

No 14 Måns Magnusson: Scalable and Efficient Probabilistic Topic Model Inference for Textual Data, 2018, ISBN 978-91-7685-288-0.

No 15 Per Sidén: Scalable Bayesian spatial analysis with Gaussian Markov random fields, 2020, 978-91-7929818-0.

\section{Linköping Studies in Information Science}

No 1 Karin Axelsson: Metodisk systemstrukturering- att skapa samstämmighet mellan informationssystemarkitektur och verksamhet, 1998. ISBN 9172-19-296-8.

No 2 Stefan Cronholm: Metodverktyg och användbarhet en studie av datorstödd metodbaserad systemutveckling, 1998, ISBN 9172-19-299-2.

No 3 Anders Avdic: Användare och utvecklare - om anveckling med kalkylprogram, 1999. ISBN 91-7219606-8.

No 4 Owen Eriksson: Kommunikationskvalitet hos informationssystem och affärsprocesser, 2000, ISBN 917219-811-7.

No 5 Mikael Lind: Från system till process - kriterier för processbestämning vid verksamhetsanalys, 2001, ISBN 91-7373-067-X.

No 6 Ulf Melin: Koordination och informationssystem i företag och nätverk, 2002, ISBN 91-7373-278-8.

No 7 Pär J. Ågerfalk: Information Systems Actability - Understanding Information Technology as a Tool for Business Action and Communication, 2003, ISBN 917373-628-7.

No 8 Ulf Seigerroth: Att förstå och förändra systemutvecklingsverksamheter - en taxonomi för metautveckling, 2003, ISBN 91-7373-736-4.

No 9 Karin Hedström: Spår av datoriseringens värden Effekter av IT i äldreomsorg, 2004, ISBN 91-7373-9634.

No 10 Ewa Braf: Knowledge Demanded for Action Studies on Knowledge Mediation in Organisations, 2004, ISBN 91-85295-47-7.

No 11 Fredrik Karlsson: Method Configuration method and computerized tool support, 2005, ISBN 91-8529748-8.

No 12 Malin Nordström: Styrbar systemförvaltning - Att organisera systemförvaltningsverksamhet med hjälp av effektiva förvaltningsobjekt, 2005, ISBN 91-8529760-7.

No 13 Stefan Holgersson: Yrke: POLIS - Yrkeskunskap, motivation, IT-system och andra förutsättningar för polisarbete, 2005, ISBN 91-85299-43-X.

No 14 Benneth Christiansson, Marie-Therese Christiansson: Mötet mellan process och komponent - mot ett ramverk för en verksamhetsnära kravspecifikation vid anskaffning av komponentbaserade informationssystem, 2006, ISBN 91-8564322-X. 


\section{FACULTY OF SCIENCE AND ENGINEERING}

Linköping Studies in Science and Technology, Dissertation No. 2168, 2021

Department of Computer and Information Science (IDA]

Linköping University

SE-581 83 Linköping, Sweden

\section{www.liu.se}

